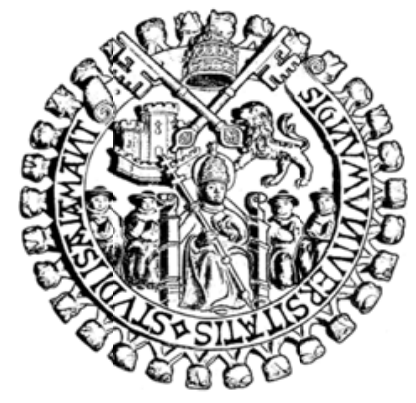

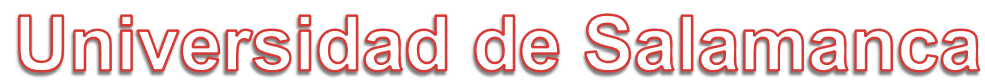 Instituto de Neurociencias de Castilla y León (INCYL)
}

\section{Modulation of in vitro and in vivo NGF-mediated functions in response to TrkA ubiquitination by Nedd4-2}

TAO YU（于涛）

SALAMANCA, 2012 



\section{Universidad de Salamanca}

Juan Carlos Arévalo

C/ Pintor Fernando Gallego, 1

Instituto de Neurociencias de Castilla y León

37007 Salamanca, Spain

Tfno: 34923294500 Ext.1871

Fax: 34923294750

Email: arevalojc@usal.es

D. Juan Carlos Arévalo Martín, Profesor Titular del Departamento de Biología Celular y

Patología y miembro del Instituto de Neurociencias de Castilla y León,

\section{CERTIFICA,}

Que D. ${ }^{a}$ Tao Yu, licenciada en Medicina, ha realizado bajo su dirección el trabajo experimental que recoge su Tesis Doctoral "Modulation of in vitro and in vivo NGFmediated functions in response to TrkA ubiquitination by Nedd4-2”.

Que ha revisado los contenidos científicos y los aspectos formales del trabajo y da su conformidad para su presentación y defensa públicas.

Para que así conste, y a los efectos oportunos, firma el presente Certificado en Salamanca, a siete de Mayo de dos mil doce.

Fdo. D. Juan Carlos Arévalo Martín 

Doña Raquel E. Rodríguez Rodríguez, Catedrática del Departamento de Bioquímica y Biología Molecular, Directora del Instituto de Neurociencias de Castilla y León,

\section{CERTIFICA,}

Que la Tesis Doctoral "Modulation of in vitro and in vivo NGF-mediated functions in response to TrkA ubiquitination by Nedd4-2”, ha sido realizada por D. a Tao Yu, licenciada en Medicina, bajo la dirección del Dr. D. Juan Carlos Arévalo Martín, y da su conformidad para que sea presentada a la Comisión de Doctorado de la Universidad de Salamanca.

Para que así conste, y a los efectos oportunos, firma el presente Certificado en Salamanca, a siete de Mayo de dos mil doce.

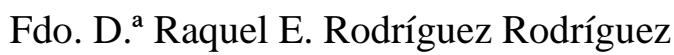





\section{Agradecimientos}

El tiempo vuela, pero siempre me acuerdo muy bien de ese día que conocí a Juan Carlos por la primera vez en la Facultad de Medicina durante la entrevista en el invierno de 2007. En ese momento empezaron mi vida y mi investigación en Salamanca. Ahora, voy a poner un punto para terminar esta época con la tesis, sin embargo, esta experencia no la voy a olvidar en toda mi vida. Durante este tiempo, he mejorado mucho mi profesión, y he recibido muchas ayudas y apoyos tanto a nivel de trabajo como personal. Quisiera, con estas lineas, poder expresar mis agradecimientos de algún modo.

En primer lugar, a mi director de tesis, el Profesor Juan Carlos Arévalo. Es un honor ser su primer estudiante de doctorado. He aprendido tantas cosas con él, con sus enseñanzas, sugerencias y pasión por la investigacion que me va a influir durante toda mi vida. Además, es una persona muy buena, aunque pocas veces entiendo sus chistes, pero su apoyo y ánimo personal me han ayudado tanto, que sin ellos nunca hubiera podido terminar la tesis.

También agradezco a la Profesora Raquel E. Rodríguez por sus colaboraciones en el proyecto, su apoyo tan amable y sus palabras tan optimistas que me han ayudado tanto para pasar los momentos duros.

A la Profesora Concepción Lillo, ha sido un placer compartir este tiempo con ella en su laboratorio. Y, a todos los profesores de Master y del INCYL.

A mis compañeros de trabajo, por todas sus ayudas, cooperaciones y el ambiente tan agradable de trabajo que proponen: a Laura, una chica muy simpática y enérgica; a Saray, la chica más jóven y considerada; a Begoña, que es muy divertida y le gusta ayudar a todos; a Saúl, el chico más majo y simpático; a Antonio, que cuenta chistes muy buenos. Aquí quería dar las gracias especiamente a Laura, porque fue la que me ayudó mucho con la disección de neuronas. Muchas gracias! 
A Ana y Cristina, por su ayuda técnica. A las personas de animalario y del confocal: David, Carmen, Elena, Sara, y Javi, por toda su ayuda y enseñanza. A la conserje Carmen, por sus atenciones y porque cada vez que entro en el INCyL siempre veo una cara simpática.

A mis amigos, a mis compañeros del Instituto y del Máster, gracias a vosotros por ayudarme a conocer la vida española, he podido ver una manera de vivir distinta y bonita. Por vuestra compañia, gracias a ella no me siento muy sola aunque este tan lejos de mi casa. Gracias Paula, Hua, Yamin, Carbiñe, Celia, Janurary, Alex, Enrique, Flora, Cheryl, Yanneri, Blanca, Roger, Katty, Rosario, Azu, Fátima, Marta, etc... Lo he pasado muy bien con vosotros!

A mi familia, por vuestro amor, ánimo y apoyo incondiconal. A mi marido, Xi, por todo su amor, apoyo, consideración y paciencia. Gracias porque siempre ha estado a mi lado dándome ánimos cuando me he caído. Muchas Gracias! 感谢我的父母和家人，感谢您们无限的，永无止境的关爱，鼓励和支持！

Al final quería dar gracias al VII Programa Marco-Marie Curie IRG por el apoyo económico que me ha proporcionado para realizar esta investigación y gracias a los miembros del tribunal y a todos los que han leído mi tesis, por su tiempo, interés y comentarios.

Agradezco a todas las personas que me han ayudado, aquellas que he mencionado y aquellas que no he mencionado sus nombres, incluso las que me han ayudado sin saber sus nombres. Gracias! Amo a España y la gente tan simpática y el Jamón! 


\section{Abbreviations}

aa

Ab

ACh

APS

ARMS

BDNF

BSA

CGRP

CIPA

CNS

CR

CREB

DAG

DMEM

DRG

DTT

DUB

E1

E2

E3

EDTA

EE

EEA1

EGF

EGFR

$\mathrm{ENaC}$

ESCRT

FBS

FRS2

GEF

GFP

Glu

Grb2

HECT

HRP

IF

Ig

Ig-C2

IP

IP3

K amino acid

Antibody

acetylcholine

Ammonium persulfate

Ankyrin Repeat-rich Membrane-Spanning protein

Brain Derived Neurotrophic Factor

Bovine Serum Albumin

Calcitonin Gene Related Peptide

Congenital Insensitivity to Pain with Anhydrosis

Central Nervous System

Cysteine Repeats

Cyclic Adenosine Monophosphate (c-AMP) Response Element Binding

Protein

Diacylglycerol

Dulbecco's Modified Eagle Medium

Dorsal Root Ganglia

Dithiothreitol

Deubiquitylating Enzyme

Ubiquitin Activating Enzyme

Ubiquitin Conjugating Enzyme

Ubiquitin Ligase

Ethylenediaminetetraacetic acid

Early Endosome

Early Endosome Antigen 1

Epidermal Growth Factor

Epidermal Growth Factor Receptor

Epithelial $\mathrm{Na}$ Channel

Endosomal Sorting Complex For Transport

Fetal Bovine Serum

Fibroblast Growth Factor Receptor Substrate-2

Guanine nucleotide Exchange Factors

Green Fluorescent Protein

Glutamate

Growth Factor Receptor Bound Protein 2

Homologous to E6AP Carboxy Terminus

Horseradish Peroxidase

Immunofluorescence

Immunoglobulin

Immunoglobulin-like C2 type domains

Immunoprecipitation

Inositol Trisphosphate

Lysine 


\begin{tabular}{|c|c|}
\hline Kidins220 & Kinase D-interacting substrate, $220 \mathrm{kDa}$ \\
\hline LE & Late Endosome \\
\hline MAPK & Mitogen-Activated Protein Kinases \\
\hline MEK & Activate Mitogen-Activated Protein Kinase Kinase \\
\hline MEM & Minimum Essential Medium \\
\hline MVB & Multivesicular Bodies \\
\hline $\mathrm{NaF}$ & Sodium Fluoride \\
\hline Nedd4 & $\begin{array}{l}\text { Neural precursor cell Expressed Developmentally Down-regulated } \\
\text { protein } 4\end{array}$ \\
\hline NGF & Nerve Growth Factor \\
\hline NGS & Normal Goat Serum \\
\hline NT & Neurotrophin \\
\hline p75NTR & p75 neurotrophin receptor \\
\hline PCR & Polymerase Chain Reaction \\
\hline PDK1 & Phosphoinositide Dependent Kinase 1 \\
\hline PDL & Poly-D-Lysine \\
\hline Pen/Strep & Penicillin/Streptomycin \\
\hline PFA & Paraformaldehyde \\
\hline $\mathrm{PI} 3 \mathrm{~K}$ & Phosphatidylinositol-3-kinase \\
\hline PKC & Protein Kinase C \\
\hline PLC-Y & Phospholipase C gamma \\
\hline PMSF & Phenylmethylsulfonyl fluoride \\
\hline PNS & Peripheral Nervous System \\
\hline PPXY & Proline Proline X Tyrosine \\
\hline RE & Recycling Endosome \\
\hline RING & Really Interesting New Gene \\
\hline RSK & Ribosomal S6 Kinase \\
\hline RTKs & Receptor Tyrosine Kinases \\
\hline RT-PCR & Reverse Transcriptase Polymerase Chain Reaction \\
\hline SCG & Superior Cervix Ganglia \\
\hline SDS & Sodium Dodecyl Sulfate \\
\hline SH2 & Src Homology 2 \\
\hline SP & Substance P \\
\hline Sulfo-NHS-SS-biotin & Sulfosuccinimidyl-2-[biotinamido]ethyl-1,3-dithiopropionate \\
\hline TEMED & $\mathrm{N}, \mathrm{N}, \mathrm{N}$ ', N'-tetramethylethylenediamine \\
\hline TG & Trigeminal Ganglia \\
\hline TNFR & Tumor Necrosis Factor Receptor \\
\hline TRAF6 & TNF Receptor Associated Factor 6 \\
\hline Trk & Tropomyosin-Related Kinase \\
\hline TRP & Transient Receptor Potential channel \\
\hline Tyr & Tyrosine residues \\
\hline
\end{tabular}




\section{Contents}

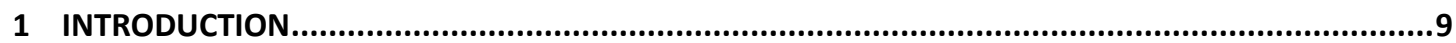

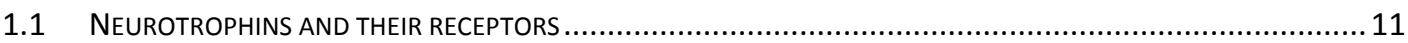

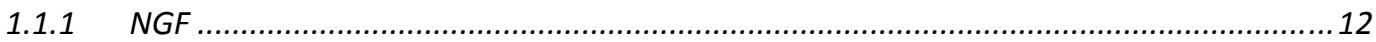

1.1.2 The TrkA neurotrophin receptor......................................................................... 13

1.1.3 The p75NTR neurotrophin receptor ................................................................ 14

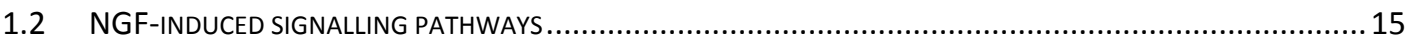

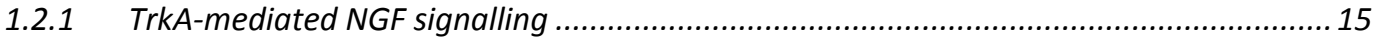

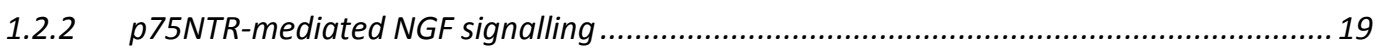

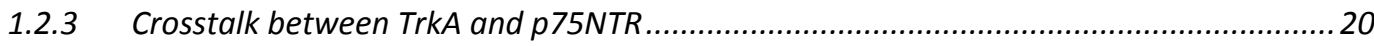

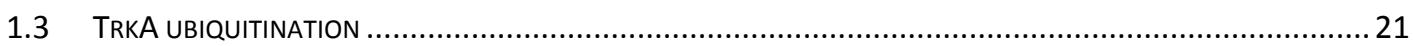

1.3.1 Ubiquitination and E3 ubiquitin ligases ....................................................... 21

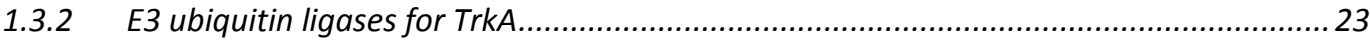

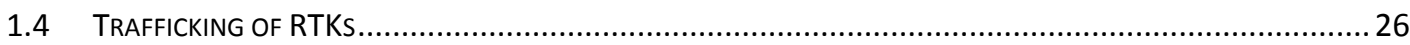

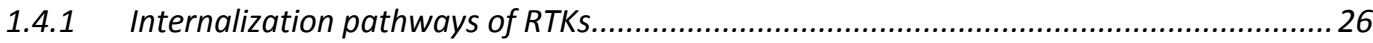

1.4.2 Factors that modulate receptor trafficking ........................................................... 28

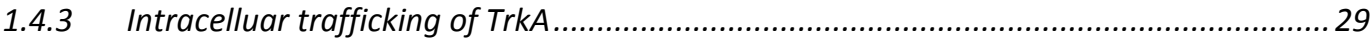

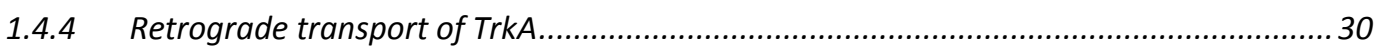

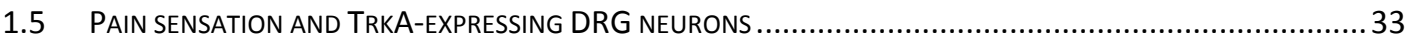

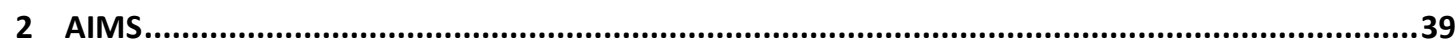

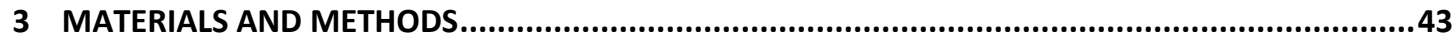

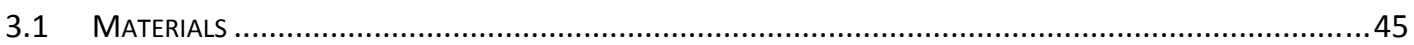

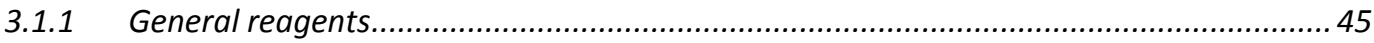

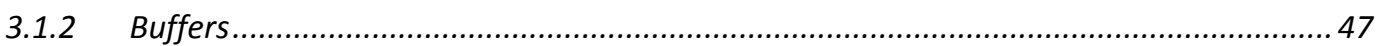

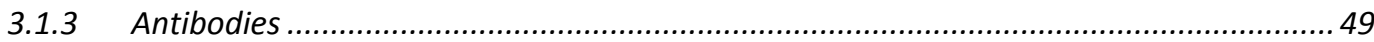

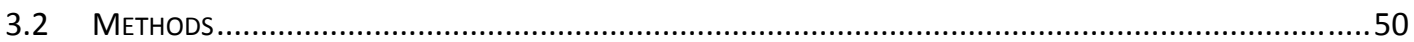

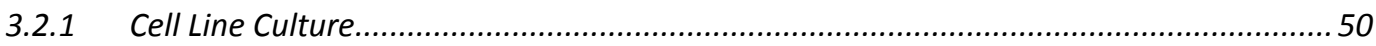

3.2.2 HEK293 cell transfection using Calcium Phosphate .................................................50 


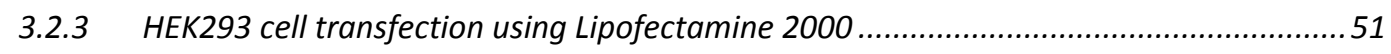

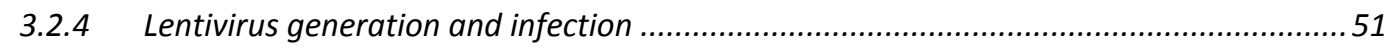

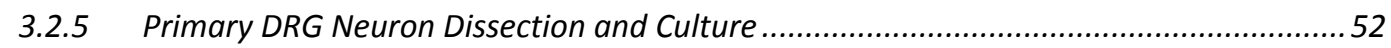

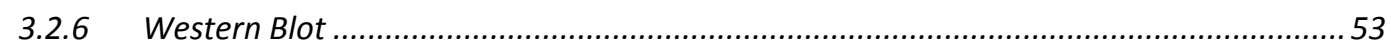

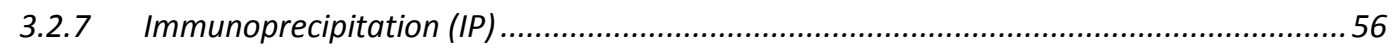

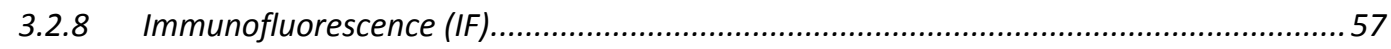

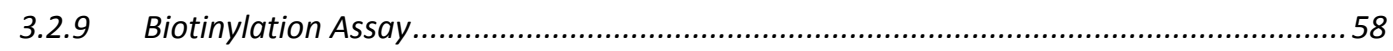

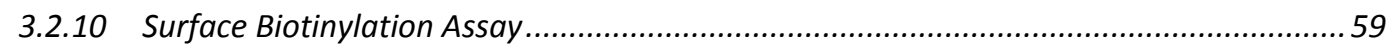

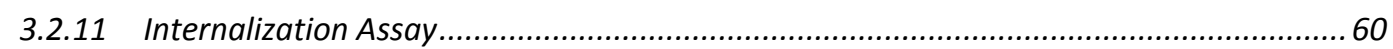

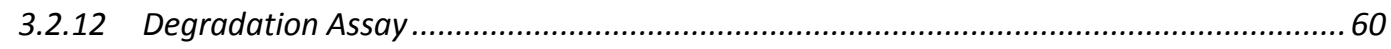

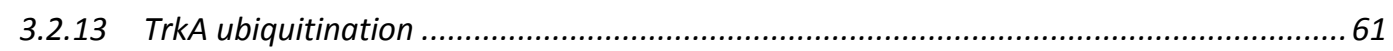

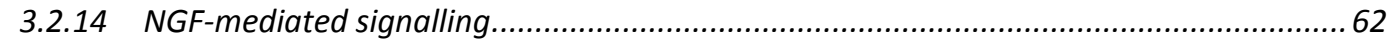

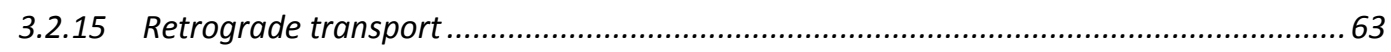

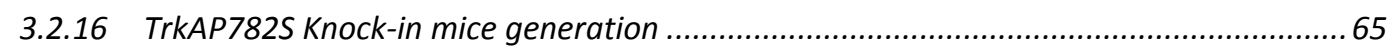

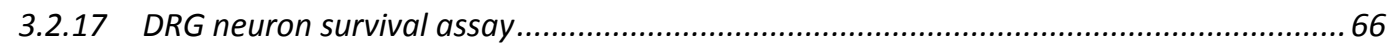

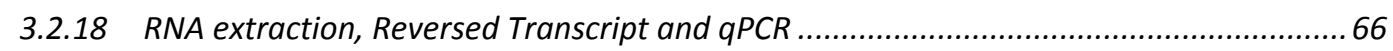

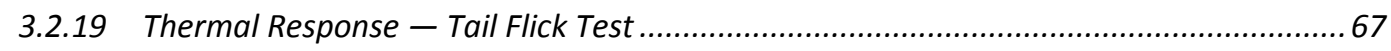

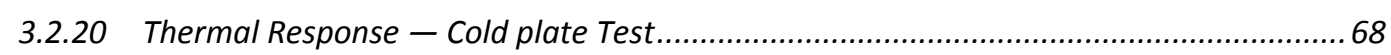

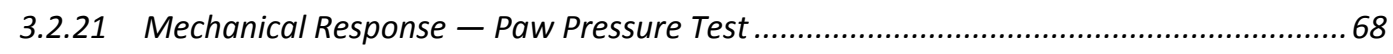

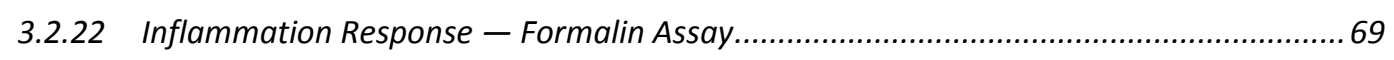

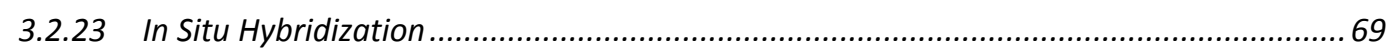

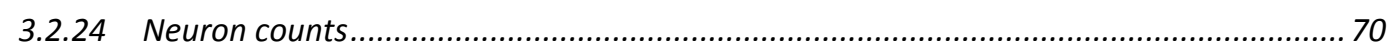

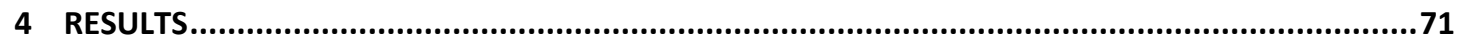

4.1 PART ONE: RESULTS FROM IN VITRO-CULTURED RAT DRG NEURONS .............................................. 73

4.1.1 Nedd4-2 regulates total- and surface-TrkA levels................................................ 73

4.1.2 Nedd4-2 modulates TrkA levels through its degradation ......................................... 75

4.1.3 TrkA trafficking at early and late endosomes is regulated by Nedd4-2 ....................... 79

4.1.4 Increased TrkA recycling to the plasma membrane by Nedd4-2 depletion......................82

4.1.5 Nedd4-2 modulates TrkA ubiquitination in early endosomes and late endosomes.........85 
4.1.6 Nedd4-2 depletion increases TrkA signalling in rat DRG neurons.

4.1.7 Nedd4-2 depletion enhances the retrograde transport of TrkA in DRG neurons............88

4.1.8 Nedd4-2 depletion increases the survival of NGF-dependent DRG neurons ...................90

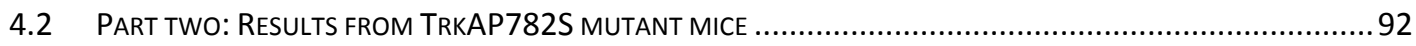

4.2.1 Generation of a knock-in (KI) mouse model expressing TrkAP782S...........................92

4.2.2 Reduced amounts of TrkAP782S and TrkAP782S mRNA in KI DRGs...........................95

4.2.3 Impaired degradation of TrkAP782S in response to NGF treatment ...........................97

4.2.4 Enhanced surface TrkA in DRG neurons from KI mice with NGF treatment.................100

4.2.5 Decreased ubiquitination of TrkAP782S in the KI DRG neurons................................... 102

4.2.6 Enhanced TrkA signalling in DRG neurons from KI mice ....................................... 102

4.2.7 Enhanced survival of DRG neurons in vitro and in vivo from KI mice........................... 103

4.2.8 Enhanced sensitivity to acute noxious thermal stimuli in KI mice..............................105

4.2.9 WT and KI mice respond similarly to acute noxious mechanical stimuli......................106

4.2.10 Enhanced response of KI mice to formalin induced inflammatory pain....................... 107

4.2.11 KI mice have a up-regulated mRNA levels of pain-related neuropeptides...................109

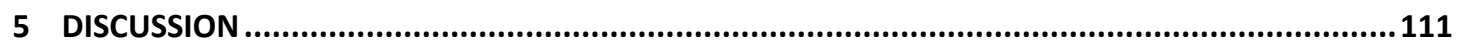

5.1 Nedd4-2 REgUlATES TRKA tRAfFiCKING IN THE EARLY AND LATE ENDOSOMAL COMPARTMENTS ................113

5.2 NEDD4-2 REGULATES TRKA INTRACELLULAR TRAFFICKING BY MODULATING ITS UBIQUITINATION LEVEL IN EARLY

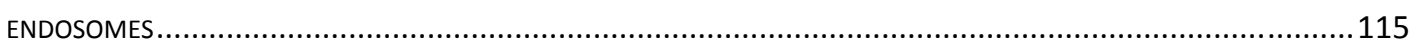

5.3 IMPLICATIONS Of ALTERATIONS IN THE PROPER TRKA TRAFFICKING CAUSED BY NEDD4-2 ........................117

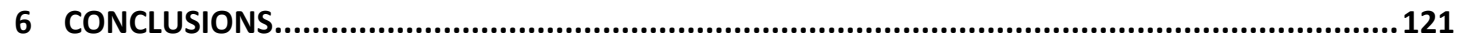

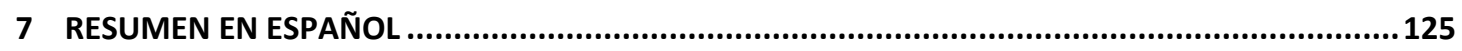

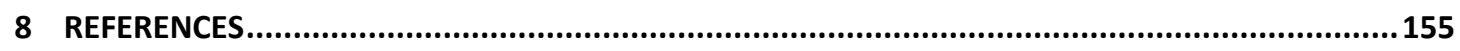

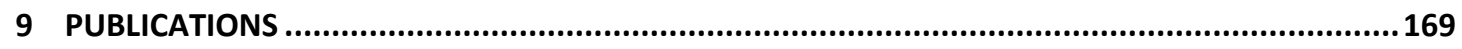


1 Introduction 


\subsection{Neurotrophins and their receptors}

Neurotrophins are growth factors that include Nerve Growth Factor (NGF), Brain-Derived Neurotrophic Factor (BDNF), Neurotrophin-3 (NT-3) and Neurotrophin-4 (NT-4). They exert different functions, such as in cell survival, proliferation, differentiation, axonal growth and synaptic plasticity through binding to their receptors. There are two kinds of receptors for neurotrophins: Tropomyosin-Related Kinase (Trk) neurotrophin receptors and p75 neurotrophin receptor (p75NTR) (Rodriguez-Tebar et al. 1990; Chao 2003). Trk neurotrophin receptors, which consist of TrkA, TrkB and TrkC, belong to the Receptor Tyrosine Kinases (RTKs) family. Different neurotrophins bind specifically to Trk neurotrophin receptors: NGF to TrkA, BDNF and NT4 to TrkB, and NT-3 to TrkC (Figure Intr1) (Huang et al. 2003; Arevalo et al. 2006c). Unlike Trk, all neurotrophins bind to p75NTR, which belongs to the Tumor Necrosis Factor Receptor (TNFR) superfamily.

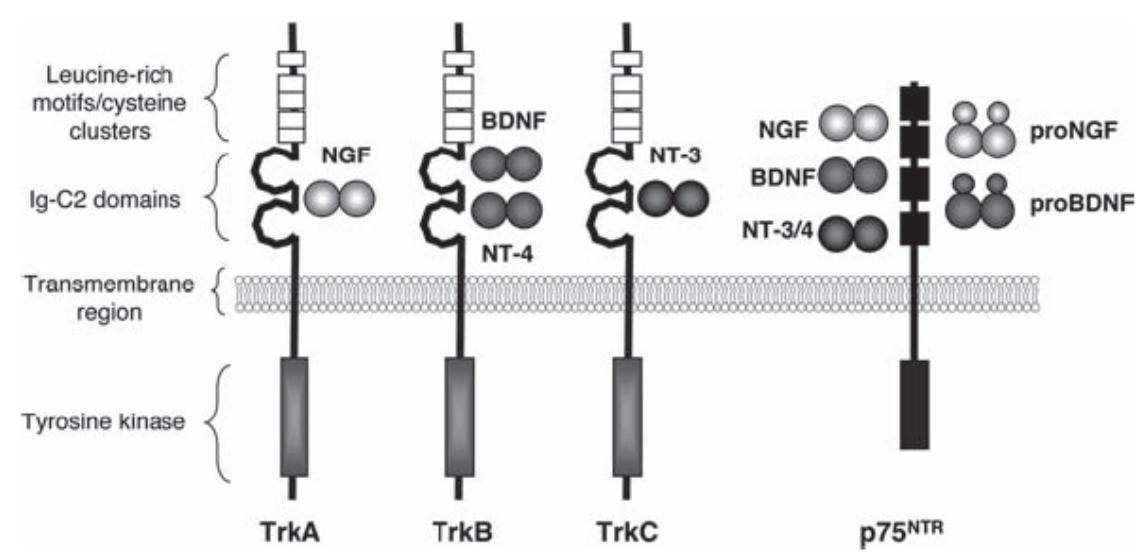

Figure Intr1. Neurotrophins and their receptors (Arevalo et al. 2006c)

Neurotrophins are dimers that bind with equal affinity to p75NTR, and specifically to Trk neurotrophin receptors. Neurotrophins are synthesized as pro-proteins that bind exclusively to p75NTR. Trk neurotrophin receptors contain an extracellular domain composed of three leucine-rich motifs flanked by two cysteine clusters, two immunoglobulin-like C2 type domains (Ig-C2), a single transmembrane domain, and a cytoplasmic region with a kinase domain. The extracellular domains of Trk neurotrophin receptors are involved in ligand binding and also in the prevention of the spontaneous dimerization of the receptor in the absence of ligand. 
The binding of neurotrophins to Trk neurotrophin receptors causes dimerization, activation and signalling transduction in the cytoplasm and therefore promotes and modulates the survival and axonal growth of different neuronal populations during development (Huang et al. 2001). Additionally, they have also been implicated in pain sensation, neuropsychiatric disorders, mood disorders, depression, bipolar disease (Duman 2004) as well as in neurodegenerative diseases such as Alzheimer's, Huntington's and Parkinson's disease (Allen et al. 2006).

\subsubsection{NGF}

NGF was discovered by Rita Levi-Montalcini in the 1950s as a small secreted protein (about $13 \mathrm{kDa}$ ) playing a role in maintaining the survival and growth of neurons. Prior to the availability of cloning techniques, the mouse submandibular gland was the richest source of NGF, since few tissues provide sufficient amounts for NGF isolation. Neurotrophins are synthesized as precursors or pro-neurotrophins, which are cleaved to produce the mature proteins. Both pro-neurotrophins and mature neurotrophins have biological functions, but differ considerably from each other. For example, the unprocessed precursor of the NGF neurotrophin, proNGF, has been suggested to be a death-inducing ligand for the neurotrophin receptor p75NTR (Lee et al. 2001a; Harrington et al. 2004), while NGF is necessary for neuronal survival (Levi-Montalcini 1987).

In the nervous system, NGF is expressed in both neurons and glial cells (Shelton et al. 1986; Fahnestock 1991). By the reverse transcriptase polymerase chain reaction (RT-PCR), the highest concentrations of NGF mRNA were found in the cortex and hippocampus, which are the major targets of the NGF-responsive cholinergic neurons of the basal forebrain nuclei. Moreover, glial cells such as astrocytes and microglia have been found to strongly up-regulate the expression of NGF after local tissue injury and inflammation. The expression of NGF is not limited to the nervous system; the skin is also a rich source of NGF and the epidermis is a recognized site of NGF expression (Di Marco et al. 1993). During development, skin-derived NGF directs innervation and maintains the survival of sensory neurons, whereas in adulthood the NGF produced by the skin is not in 
charge of neuronal survival. Instead, it has been shown to be up-regulated in mast cells, keratinocytes, fibroblasts, lymphocytes and macrophages after skin injury or inflammation (Di Marco et al. 1993), indicating its involvement in inflammatory and immune responses, and in the maintenance of pain hypersensitivity (Pezet et al. 2006).

\subsubsection{The TrkA neurotrophin receptor}

TrkA was originally discovered as a rearrangement of non-muscle tropomyosin with an unknown tyrosine kinase (Martin-Zanca et al. 1986) and was subsequently identified as a receptor for NGF (Kaplan et al. 1991b; Klein et al. 1991). Trk neurotrophin receptors are single transmembrane catalytic receptors with intracellular tyrosine kinase activity. They contain an extracellular domain composed of three leucine-rich motifs flanked by two cysteine clusters, two immunoglobulin-like C2 type domains (Ig-C2), a single transmembrane domain followed by the juxtamembrane region, and a cytoplasmic region with a tyrosine kinase domain (Figure Intr1) (Arevalo et al. 2006c). The cytoplasmic kinase domain includes several tyrosine residues (Tyr) such as Tyr490, Tyr674/675, Tyr751 and Tyr785, whose phosphorylation is necessary for the docking of different adaptor molecules and the induction of downstream signalling pathways.

TrkA is expressed in a variety of cell types, neurons and non-neuronal cells. TrkA is found in certain sensory neurons in the Dorsal Root Ganglia (DRG), the Trigeminal Ganglia (TG) and in the sympathetic neurons in the Superior Cervix Ganglia (SCG) in the Peripheral Nervous System (PNS) (Martin-Zanca et al. 1990) and in a small subset of cholinergic neurons in the caudatoputamen and the basal forebrain in the Central Nervous System (CNS) (Martin-Zanca et al. 1990). The expression of TrkA is not restricted to neurons. It has been observed in glial cells such as the Schwann cells in the PNS, astrocytes and microglia cells in the CNS (Savaskan et al. 2000) and in gliomas and astrocytomas (Kramer et al. 1996). In addition, it has also been found in defensive keratinocytes, immune mast cells, and macrophage cells (Tam et al. 1997; Terenghi et al. 1997).

TrkA, together with accessible NGF, plays an important role in neuronal survival during development and nociception in adulthood. The survival of TrkA-expressing sensory 
neurons in DRGs in the PNS (Crowley et al. 1994; Smeyne et al. 1994) and cholinergic neurons in the CNS depends on their ability to access target-derived NGF (Sofroniew et al. 1990). A considerable body of evidence has shown the importance of NGF-TrkA in pain sensation during adulthood, since mice with a null mutation for either NGF or TrkA are unresponsive to painful stimuli (Crowley et al. 1994; Smeyne et al. 1994).

\subsubsection{The p75NTR neurotrophin receptor}

Although TrkA is the best studied receptor for NGF, p75NTR was the first one identified (Chao et al. 1986). p75NTR is a transmembrane glycoprotein protein of about $75 \mathrm{KDa}$ that belongs to the TNFR superfamily. Like other TNFRs, p75NTR is composed of four cysteine repeats (CR1-CR4) in the extracellular domain that form the ligand-binding domain, a short transmembrane spanning domain, and a short intracellular domain (Arevalo et al. 2006c). Unlike TrkA, p75NTR has no intrinsic catalytic activity; cytoplasmic interactors must be recruited to produce the signals (Hasegawa et al. 2004), which include NADE (Bex3), NRIF, NRAGE, SC1, RhoA, TRAF, FAP-1, etc.

The expression patterns of p75NTR and TrkA overlap extensively in specific neurons in the CNS and in sensory and sympathetic neurons in the PNS (Davies 2000). However, unlike the cell survival and proliferation induced by NGF-TrkA signalling, the NGF-p75NTR complex induces apoptosis. In addition, although proNGF was initially considered as an inactive form of NGF, in fact it can bind to p75NTR and induce apoptotic cell death even in the presence of TrkA (Lee et al. 2001b). Similar to TrkA, the level of p75NTR may be modulated by the inflammatory condition. For example, (1) in Alzheimer's patients, cortical neurons express p75NTR, where p75NTR is not normally detected (Mufson et al. 1992); (2) after nerve lesions or removal of axonal contacts, an up-regulation of p75NTR has been observed in Schwann cells (Lemke et al. 1988), and (3) in multiple sclerosis p75NTR is has been found to be increased in oligodentrocytes, microglia and macrophages (Lee et al. 2001a). 


\subsection{NGF-induced signalling pathways}

NGF exerts its biological functions through binding to TrkA and p75NTR neurotrophin receptors, which trigger different signalling pathways and elicit different biological functions. While NGF-TrkA signalling causes cell survival and is involved in nociception, NGF-p75NTR signalling leads to cell death. This complexity and even the opposite biological functions are due to the different signalling pathways that each receptor uses. Here we review the signalling pathways induced by each NGF receptor and their cross-talk.

\subsubsection{TrkA-mediated NGF signalling}

Upon NGF binding, TrkA neurotrophin receptor dimerizes and is transphosphorylated in the intracellular tyrosine residues, like other RTKs. Among them, the activation of Tyr490 and Tyr785 are those best studied and form the major docking sites for adaptor proteins, such as Src Homology 2 (SH2)-containing protein Shc, fibroblast growth factor receptor substrate-2 (FRS2) and Phospholipase C gamma (PLC-y) (Figure intr2). Three major signalling pathways are initiated: the Mitogen-Activated Protein Kinase (MAPK) pathway, the Phosphatidyllnositol-3-kinase (PI3K)-AKT pathway, and the PLC-y protein kinase C (PKC) pathway (Arevalo et al. 2006c).

\section{The MAPK pathway}

Phosphorylation at the Tyr490 residue in the cytoplasmic domain of TrkA by NGF recruits the $\mathrm{SH} 2$ domain-containing adaptor protein Shc to form a complex with Growth factor Receptor Bound protein 2 (Grb2), the Guanine nucleotide Exchange Factor (GEF) SOS and Gab1/2. Activation of SOS promotes the exchange of GDP for GTP in Ras, (a small GTPase) to make Ras active and then activates a cascade including the protein kinase activity of Raf kinase, the mitogen-activated protein kinase kinase (MEK), and MAPK. Activated MAPK transduces the ligand-induced signalling to the nucleus to modulate gene transcription (Aftab et al. 1997) (Figure Intr2). The MAPK is a pathway induced by the 
activation of different RTKs. However, the biological functions differ considerably, depending on the different ligands and receptors. For example, PC12 cells, a rat adrenal pheochromocytoma cell line, respond to both NGF and Epidermal Growth Factor (EGF) through TrkA and Epidermal Growth Factor Receptor (EGFR), respectively, triggering the MAPK signalling pathway. However, EGF causes cell proliferation, while NGF induces cell differentiation. Why does the same signalling bring about different outcomes? It has been reported that besides triggering a transient MAPK signalling pathway similar to that induced by EGF through Ras activation, the binding of NGF to TrkA leads to a sustained MAPK signalling pathway by the activation of the small GTPase Rap1 (York et al. 1998). Although both EGF and NGF cause a transient activation of Ras, only NGF induces the activation of Rap1, whose activation is sustained for hours (York et al. 1998). Moreover, it has been proposed that endosomes are a site from which NGF induces the prolonged activation of MAPK, since pre-treatment of PC12 cells with brefeldin A, which disrupts the Golgi and endosomal compartments, has little effect on Ras activation but strongly inhibits NGF-induced Rap1 activation and prolonged MAPK activation (Wu et al. 2001; Arevalo et al. 2006a). Nevertheless, how TrkA activation induces Rap activation is not well understood. Arévalo et al. reported that an unusual ankyrin-rich transmembrane protein (ARMS/kidins220) acts as a major and neuronal-specific platform for prolonged MAP kinase signalling by neurotrophins (Arevalo et al. 2004). They found that ARMS is closely associated with Trk neurotrophin receptor tyrosine kinases (Chang et al. 2004), but not the EGF receptor, through interactions between the transmembrane domains of Trk and ARMS. Upon neurotrophin treatment, ARMS is rapidly tyrosine-phosphorylated and provides a docking site for the complex of an adaptor protein, CrkL, and a guanine nucleotide exchange factor, C3G (the CrkL-C3G complex), resulting in Rap1-dependent sustained MAPK activation (Figure Intr2) (Arevalo et al. 2004; Arevalo et al. 2006a). Other groups have proposed that FRS2 would be involved in Rap1 activation (Meakin et al. 1999; Kao et al. 2001).

Activated MAPK is capable of modulating gene expression by phosphorylating transcription factors such as c-Jun (Neuberg et al. 1989), c-Fos (Neuberg et al. 1989), and 
Elk1 (Dalton et al. 1992). In addition, the protein kinase Ribosomal S6 Kinase is another substrate of activated MAPK. Phosphorylated RSK leads to its nuclear translocation and consequently the phosphorylation of Cyclic Adenosine Monophosphate (c-AMP) Response Element-Binding Protein (CREB), which then leads to c-Fos transcription. Activated CREB also regulates bcl-2 transcription, thereby functioning in controlling cell death and survival (Meller et al. 2005).

\section{The PI3KIAKT pathway}

Engagement of Shc to TrkA upon activation induces the formation of a complex with Grb2 and Gab1/2 that can activate PI3K. PI3Ks are a family of lipid kinases defined by their ability to phosphorylate the $3^{\prime}-\mathrm{OH}$ group of the inositol ring in lipid phosphoinositides (Ptdlns) (Blume-Jensen et al. 2001; Jimenez et al. 2002). Thus, the, activation of PI3K could transform Ptdlns $(4) P$ or $\operatorname{Ptdlns}(4,5) P_{2}$ to $P \operatorname{tdlns}(3,4) P_{2}$ or $\operatorname{Ptdlns}(3,4,5) P_{3}$, respectively, on the plasma membrane. These Ptdlns recruit phosphoinositide-dependent kinase 1 (PDK1) and AKT to the plasma membrane through the PH domain and then AKT is phosphorylated and activated by PDK1. AKT is a key downstream effector of PI3K and its activation regulates several transcription factors and regulatory proteins to modulate cell survival, proliferation and other biological functions. For example, the role of Akt in cell survival is through the regulation of its downstream pro-apoptotic and pro-survival effectors: (1) the phosporylation of BAD and Caspase- 9 by Akt inhibits their pro-apoptotic effects (Rothenberger et al. 1996); (2) the phosphorylation of the forkhead transcription factor FKHRL1 by Akt results in FKHRL1 retention in the cytoplasm and the inhibition of FKHRL1-dependent expression of pro-apoptotic proteins (Brunet et al. 1999); (3) the NF-kB pro-survival pathway is activated via Akt phosphorylation of the inhibitor IkB, which causes the dissociation of IKB from NF-KB, thereby allowing the translocation of NF-KB to the nucleus. Once there, it up-regulates the transcription of anti-apoptotic genes, such as the inhibitors of apoptosis proteins (IAPs) and Bcl-2 (Kim et al. 2001). 


\section{The PLC- $\gamma$ pathway}

The phosphorylation of Y785 of TrkA in response to NGF recruits PLC- $\gamma$ through an $\mathrm{SH} 2$ domain. PLC- $\gamma$ signalling is important in mediating growth factor actions both in vitro and in vivo (Choi et al. 2001). Similarly to PI3K, the recruitment of PLC- $\gamma$ to the membrane brings the enzyme to their substrates in the lipid bilayer. Activated PLC- $\gamma$ catalyzes PIP2 to form the second messenger diacylglycerol (DAG) and inositol trisphosphate (IP3) (Berridge et al. 1984; Lopes et al. 2007). DAG activates protein kinase C (PKC), while IP3 triggers calcium release from intracellular stores. Through Raf, active PKC can cause the activation of the MAPK signalling pathway.

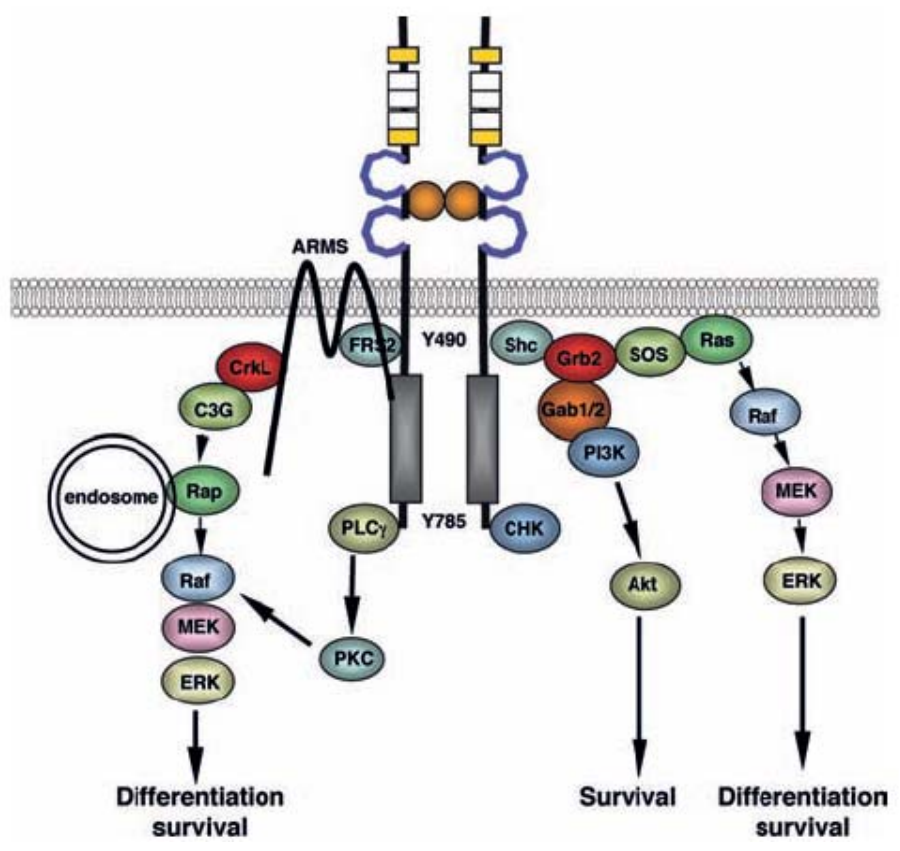

Figure Intr2. Trk receptor-mediated signalling pathways (Arevalo et al. 2006c).

Neurotrophin binding to Trk triggers its dimeration, auto-phosphorylation and recruitment of different adaptor proteins to lead the activation of various signalling pathways, such as the Ras, Rap, PI3K, and PLC-y pathways, which result in survival, neurite outgrowth, gene expression, and synaptic plasticity. 


\subsection{2 p75NTR-mediated NGF signalling}

Unlike TrkA, p75NTR does not have cytoplasmic kinase activity; its activity relies on the adaptor proteins (NADE, NRIF, NRAGE, TRAF, SC1, etc.) bound to the cytoplasmic domain (Figure Intr3). Through different adaptors, p75NTR may cause very different actions: cell death as well as cell survival, neurite elongation and growth arrest (Abrams et al. 1998; Mamidipudi et al. 2002); signalling via NADE (Mukai et al. 2000), NRIF (Casademunt et al. 1999) or NRAGE (Salehi et al. 2000) causes cell apoptosis, but conversely the recruitment of TRAF proteins leads to the activation of transcription factors such as, NF-KB and JNK, thereby promoting cell survival and differentiation (Ye et al. 1999). An interaction between p75NTR and Rho A or SC-1 in a ligand-dependent manner modulates neurite elongation and growth arrest respectively (Chittka et al. 1999; Yamashita et al. 1999).

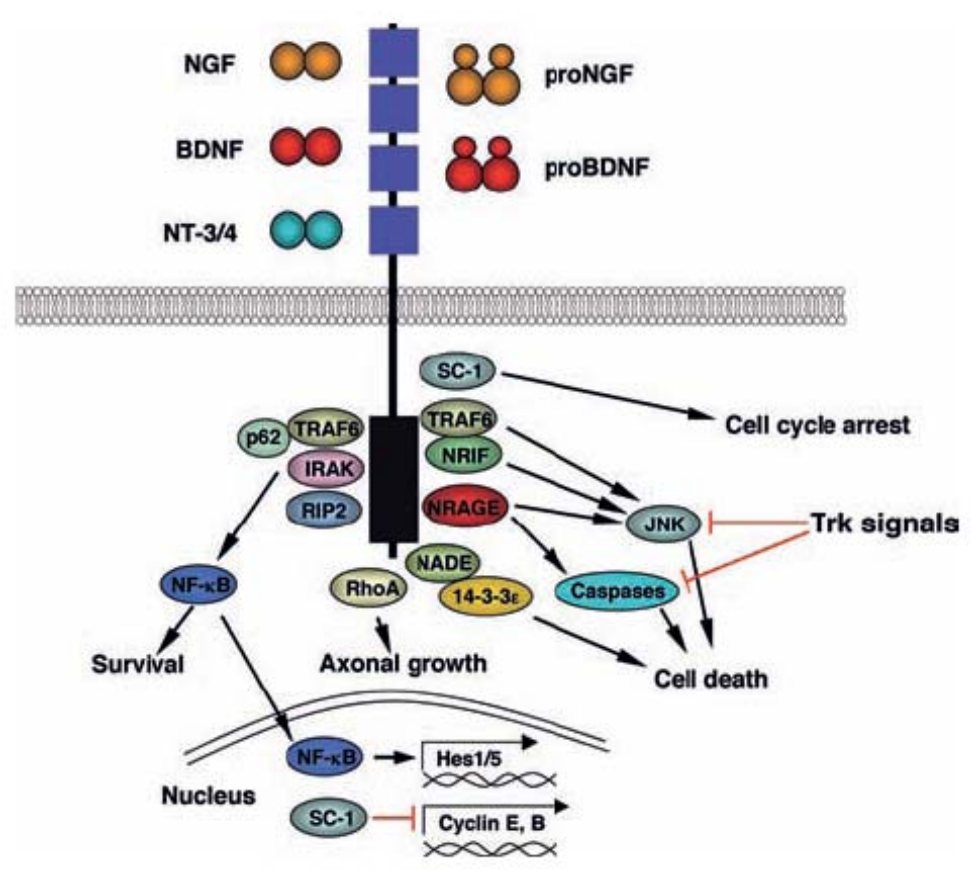

Figure Intr3. The p75NTR signalling pathway (Arevalo et al. 2006c)

Mature neurotrophins or pro-neurotrophins bind to p75NTR to trigger the activation of different signalling pathways through different adaptors that result in diverse, and at times opposite, outcomes such as survival, apoptosis, axonal growth, axonal collapse, and cell cycle arrest. 


\subsubsection{Crosstalk between TrkA and p75NTR}

NGF binds to both TrkA and p75NTR, which are co-expressed in many cells. However, these two types of receptor may lead to opposite actions: cell survival or apoptosis respectively. Thus, what will the final outcome in cells that co-express both receptors be? Do they cooperate or perform their different roles alone? Growing evidence suggests the existence of a significant cross-talk between these receptors (Figure Intr4). It has been found that p75NTR is associated with TrkA, TrkB and TrkC in cells transfected with both receptor types (Bibel et al. 1999) and the co-expression of p75NTR with TrkA causes cell survival and growth instead of cell death (Bibel et al. 1999; Jiang et al. 1999). Moreover, less NGF is needed in cells that co-express TrkA and p75NTR than in those only expressing TrkA (Hempstead et al. 1991). In addition, p75NTR knock-out mice showed a loss of nociceptive sensory neurons (Lee et al. 1992), as in NGF knock-out (Crowley et al. 1994) and TrkA knock-out mice (Smeyne et al. 1994). It therefore seems that p75NTR cooperates with TrkA and that NGF-mediated cell death through p75NTR only occurs when TrkA is absent. TrkA activation can block p75NTR-mediated cell death by several mechanisms. For example, the interaction of both receptors may disrupt the interaction of p75NTR with a death-transducing protein, in analogy with the interaction of the TNF receptor with the suppressor of the death domain protein (Jiang et al. 1999).

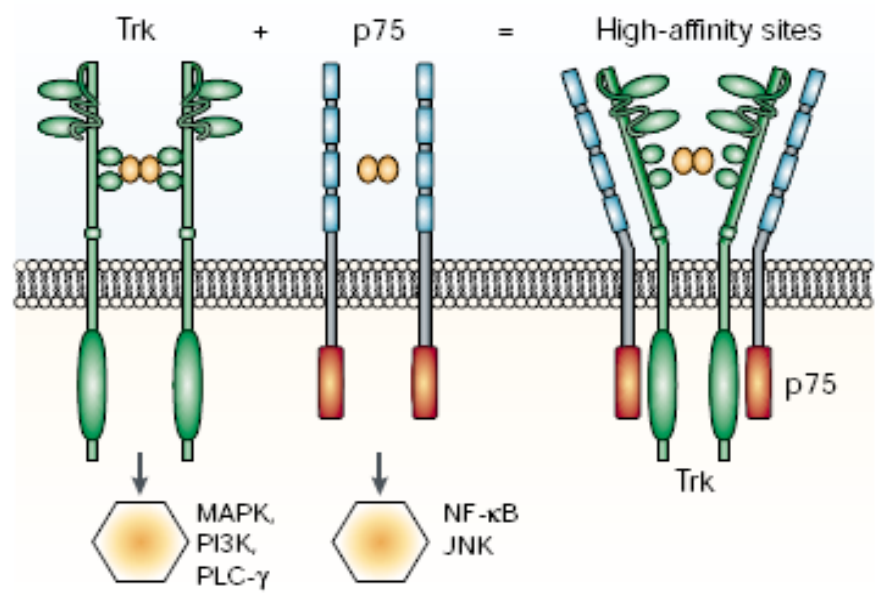

Figure Intr4. Cross-talk between TrkA and p75NTR (Chao 2003)

Interactions between Trk and p75NTR neurotrophin receptors can lead to changes in the binding affinity for neurotrophins. 


\subsection{TrkA ubiquitination}

\subsubsection{Ubiquitination and E3 ubiquitin ligases}

Ubiquitination is a post-translational modification carried out by a set of enzymes to tag ubiquitin, a 76-amino acid polypeptide, to the substrate protein. In this process, ubiquitin is covalently conjugated to the ubiquitin-activating enzyme (E1) in an ATP-dependent manner, after which it is transferred to the ubiquitin-conjugating enzyme (E2), and finally to the substrate protein through the ubiquitin-protein ligase (E3) bridge (Hershko et al. 2000) (Figure Intr5A). Ubiquitin is bound to a substrate protein through the formation of isopeptide bonds between the $\mathrm{C}$ terminus of ubiquitin and lysine $(\mathrm{K})$ present on substrates. Depending on the number of ubiquitin moieties added to the substrate, there are: (i) monoubiquitination, which is the attachment of a single ubiquitin to a protein; (ii) multi-monoubiquitination, in which more than one ubiquitin is added to the target protein and (iii) polyubiquitination, in which several moieties of ubiquitin are added (Figure Intr5B). Since ubiquitin itself contains seven lysine residues (K6, K11, K27, K29, K33, K48, and K63), the conjugated ubiquitin can serve as a substrate for the binding of the next ubiquitin to form a chain (Hershko et al. 1998).

The degradation of a protein is as crucial as its synthesis in the cellular biological functions. It ensures the elimination of signalling proteins and the proteins that participate in cell growth and cell cycle in time. If this does not occur, an unwanted protein will be accumulated and consequently this will lead to functional disorders. In certain cancers, oncogenic targets are mutated such that they are no longer subjected to ubiquitination and therefore escape from degradation and accumulate in the cell. In the nervous system, correct degradation ensures cell-surface protein turnover and substrate delivery to the proteolytic machineries that are required for both synaptic plasticity and self-renewal (Tai et al. 2008), while abnormal protein accumulation could lead to chronic neurodegenerative processes, such as Alzheimer's disease, Parkinson's disease, Lewy body dementia, and amyotrophic lateral sclerosis (Bedford et al. 2011). 
A

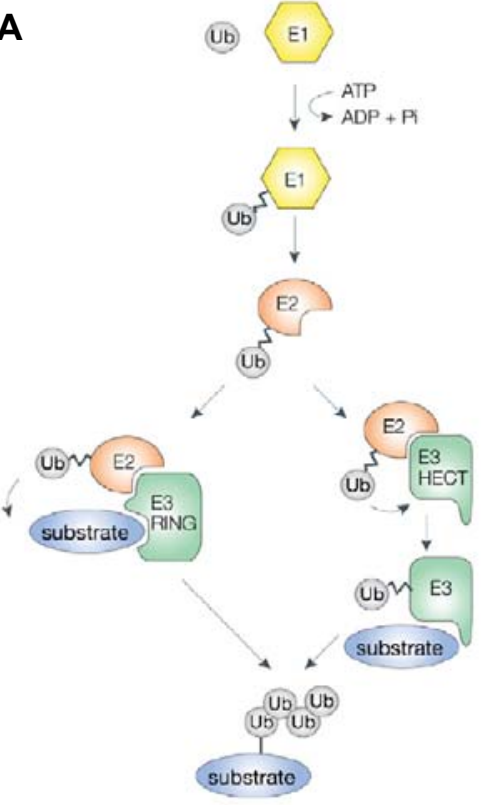

B

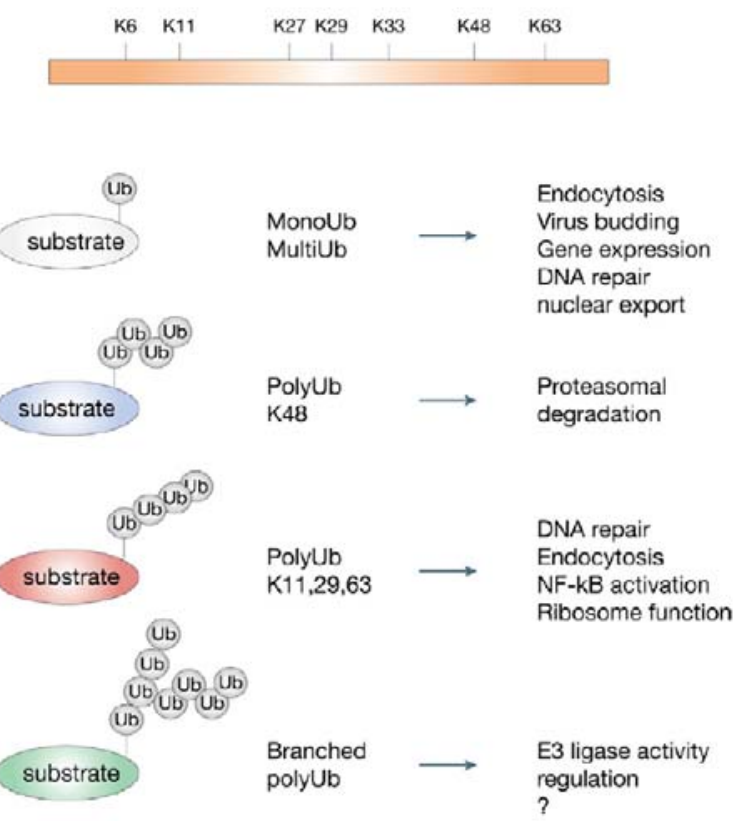

Figure Intr5. Protein ubiquitination (Woelk et al. 2007)

(A) Schematic representation of the ubiquitination process.

(B) Schematic representation of the different Ub modifications with their functional roles. The question mark indicates that the functions of branched chains are largely unknown.

Although protein ubiquitination was first identified as a tag of protein degradation, numerous lines of evidence have shown that ubiquitin labelling is not always fatal for a protein. For example, it has been found that monoubiquitination is involved in histone regulation, endocytosis, virus budding, Golgi reassembly, gene transcription, DNA repair and tumour suppression (Ikeda et al. 2008). K48 polyubiquitination mainly leads to the proteasomal degradation of substrates while, similar to monoubiquitination, K63 polyubiquitination contributes to intracellular trafficking (Hershko et al. 2000; Geetha et al. 2005; Mukhopadhyay et al. 2007). Thus, protein ubiquitination is a complex process that is still not fully understood. Different forms of ubiquitination are related to different fates of the ubiquitinated substrate proteins and hence distinct cell biological functions.

In the process of ubiquitination, much attention has focused on ubiquitin-related enzymes. Interestingly, it was found that mammalian genomes encode just two E1s, dozens of E2s, but more than 500 E3s (Kawabe et al. 2011). Moreover, while E2s share many 
well-conserved catalytic domains, E3 ubiquitin ligases only share a few conserved motifs. Therefore, E3 ubiquitin ligases are considered to carry out the important task of recognizing substrate proteins. E3 ubiquitin ligases are divided into several families based on their structural characteristics, which include (i) the Really Interesting New Gene (RING) family, such as MDM2, and c-Cbl; (ii) the Homologous to E6AP Carboxy Terminus (HECT) family, such as the neural precursor cell-expressed, developmentally down-regulated 4 (Nedd4) family; and (iii) the UFD2 homology (U-box) family. The functional differences between the RING-family and the HECT-family E3 ubiquitin ligases lie in the fact that that the RINGs E3 ubiquitin ligases add ubiquitin carried by E2s directly to the substrates, whereas the HECT E3 ubiquitin ligases form a thiol ester intermediate with ubiquitin transferred from the E2s first and then transfer the ubiquitin to the substrate (Figure Intr5A). In addition, HECT E3 ubiquitin ligases have a direct role in catalysis during ubiquitination, whereas RING and U-box E3 ubiquitin ligases facilitate protein ubiquitination. These latter two E3 ubiquitin ligase types act as adaptor-like molecules by bringing an E2 and a substrate into sufficiently close proximity to promote the ubiquitination of the substrate. While RING-type E3 ubiquitin ligases, such as MDM2 and c-Cbl, can apparently act alone, other E3 ubiquitin ligases are found as components of much larger multi-protein complexes (Ardley et al. 2005).

\subsubsection{E3 ubiquitin ligases for TrkA}

Once activated by NGF, TrkA undergoes activation and ubiquitination (Geetha et al. 2005; Makkerh et al. 2005; Arevalo et al. 2006b). Until now, several groups have reported that different E3 ubiquitin ligases are responsible for TrkA ubiquitination.

\section{TRAF6}

In 2005, Geetha et al. reported that TNF Receptor Associated Factor 6 (TRAF6) can ubiquitinate TrkA in a K63 polyubiquitinating manner (Geetha et al. 2005). TRAF6 is a docking protein with a C-terminal TRAF domain that facilitates its interaction with receptors and other adaptor proteins, while its $\mathrm{N}$-terminal domain possesses an E3 ubiquitin ligase RING motif. A cluster of zinc fingers connects the C-terminal and 
$\mathrm{N}$-terminal regions (Ishida et al. 1996). TRAF proteins are associated with and mediate signal transduction from members of the TNF receptor superfamily. For example, TRAF6 has been found to be associated with p75NTR, functioning as a signal transducer for NGF actions through p75NTR (Khursigara et al. 1999). TRAF6 forms a complex with p75NTR and p62 to ubiquitinate Trk neurotrophin receptors at K485 in the juxtamembrane region (Geetha et al. 2005). Besides ubiquitinating Trk proteins, TRAF6 has been found to be an E3 ubiquitin ligase for Akt and it seems to be essential for Akt ubiquitination, membrane recruitment, and phosphorylation upon growth factor stimulation (Namjou et al. 2012). Recently, TRAF6 has also been reported to be involved in muscle atrophy (Paul et al. 2012), in the pathogenesis of autoimmunity (Mu et al. 2011; Starczynowski et al. 2011), in CD40-mediated cell death (Jundi et al. 2012), in Huntington's disease (Zucchelli et al. 2011) and in cancer (Mu et al. 2011; Starczynowski et al. 2011).

\section{Nedd4-2}

Following the identification of TRAF6 for TrkA ubiquitination, Arévalo et al. reported that Neural precursor cell Expressed Developmentally Down-regulated protein 4-2 (Nedd4-2), a HECT E3 ubiquitin ligase, ubiquitinates TrkA (Arevalo et al. 2006b). Nedd4-2 is specifically associated with TrkA and is phosphorylated upon NGF binding. Nedd4-2 binds to TrkA by recognizing the PPXY motif, a motif that is not present in TrkB; hence, Nedd4-2 cannot bind and ubiquitinate TrkB (Arevalo et al. 2006b). Nedd4-2 belongs to the Nedd4 family, which includes nine members in humans: Nedd4-1, Nedd4-2 (Nedd4L), Itch, WWP1, WWP2, SMURF1, SMURF2, Bul1 and NedL2. All of them contain a catalytic HECT domain at the C-terminus, a $\mathrm{C} 2$ domain in the $\mathrm{N}$ terminus, and several WW domains (Figure Intr6) (Yang et al. 2010). The C2 domain translocates the protein to the membrane upon calcium binding, and the WW domains are known to bind substrate proteins containing PY motifs. The catalytic cysteine within the HECT domain is responsible for ubiquitin transfer (Chen et al. 2007). In 2011, Georgieva et al. demonstrated that the C-terminal tail hydrophobicity of TrkA regulates the strength and activity of Nedd4-2 binding and therefore controls the turnover of TrkA (Georgieva et al. 2011). Additional support for Nedd4-2 in regulating TrkA comes from a study using protein 
microarrays that identified TrkA as a binding partner of Nedd4-2 (Persaud et al. 2009). Although it is clear that Nedd4-2 binds and regulates the levels of TrkA, the mechanism by which Nedd4-2 affects TrkA is not completely understood.

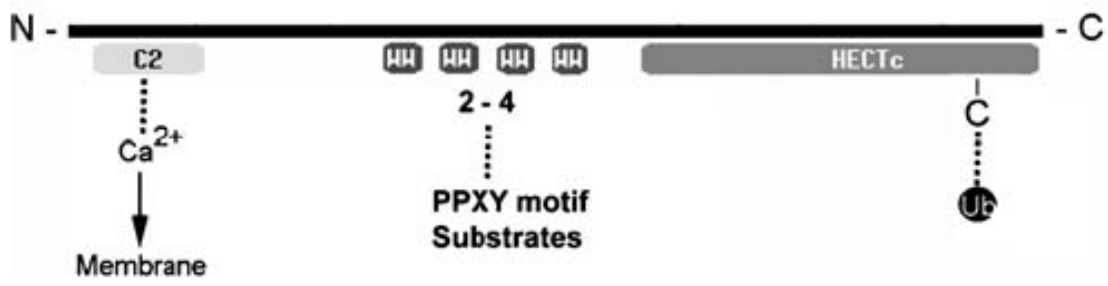

Figure Intr6. Schematic diagram of Nedd4-2 E3 ubiquitin ligases (Chen et al. 2007)

The $\mathrm{C} 2$ domain in the $\mathrm{N}$ terminus translocates the protein to the membrane upon calcium binding, and the two-to-four WW domains are known to bind substrate proteins containing PY motifs. The catalytic cysteine within the HECT domain at the C-terminus is responsible for ubiquitin transfer.

Besides ubiquitinating TrkA and regulating the survival of sensory neurons (Arevalo et al. 2006b), Nedd4-2 has also been identified as an E3 ubiquitin ligase for the Epithelial $\mathrm{Na}$ Channel $(\mathrm{ENaC})$, regulating its degradation and activity. The $\mathrm{ENaC}$ is responsible for salt and fluid re-absorption in the distal nephron, distal colon, and lung epithelia, and abnormal elevations of ENaC activity lead to hypertension (Hummler 1999). It has been found that Nedd4-2 catalyzes both the monoubiquitination and polyubiquitination of ENaC (Zhou et al. 2007). Mutations that delete or disrupt a C-terminal PY motif in the ENaC prevent the interaction of Nedd4-2 and the ENaC and increase renal Na+ absorption, causing Liddle's syndrome (an inherited form of hypertension) (Knight et al. 2006). The roles of Nedd4-2 in $\mathrm{ENaC}$ activity have also been found in the clearance of lung fluid in newborn animals. Mice knocked-out for Nedd 4-2 die perinatally with increased ENaC expression and activity in the lung, which leads to a failure to inflate the lungs, in turn resulting in an inability of the pups to breathe (Zhang et al. 2010; Boase et al. 2011). In addition, Nedd4-2 has been reported to play a protective regulatory role against the development of cystic fibrosis in lung through the regulation of ENaC function (Zhang et al. 2010). 


\section{Cbl}

In 2011, Takahashi reported that the E3 ubiquitin ligase Cbl, a well studied E3 ubiquitin ligase for EGFR (Grovdal et al. 2004; Visser Smit et al. 2009; Zhou et al. 2011), may mediate the ligand-induced ubiquitination and degradation of TrkA (Takahashi et al. 2011). They found that $\mathrm{Cbl}$ and ubiquitinated TrkA were in a complex after NGF stimulation. TrkA ubiquitination and degradation required direct interactions between $\mathrm{Cbl}$ and phosphorylated TrkA and hence the authors concluded that the ligand-induced down-regulation of TrkA is partly regulated through $\mathrm{Cbl}$. Cbl has a highly proline-rich region and a large number of potential tyrosine phosphorylation sites, which could mediate its interactions with the $\mathrm{SH} 3$ and $\mathrm{SH} 2$ domains of signalling proteins, respectively (Blake et al. 1991).

\subsection{Trafficking of RTKs}

Once activated upon ligand binding at the plasma membrane, RTKs are internalized into early endosomes (EE). From here, internalized receptors can be transported to late endosomes (LE) and lysosomes to attenuate receptor-mediated signalling pathways through degradation, or they can be transported back to the recycling endosomes (RE) to be reinserted into the cell surface for functional resensitization (Sorkin et al. 2009). Strong evidence shows that during intracellular trafficking before being degraded receptors are still active in endosomes (Wiley et al. 2001). Thus, cytoplasmic receptor trafficking controls the activation and downstream signalling pathways.

\subsubsection{Internalization pathways of RTKs}

Several pathways have been identified as being responsible for the internalization of RTKs: clathrin-dependent endocytosis, caveolin-dependent endocytosis and clathrin- and caveolin-independent pathways (Aguilar et al. 2005; Sigismund et al. 2005). In the clathrin-dependent pathway, receptors and their bound ligands are invaginated in clathrin-coated vesicles, while in the caveolin-dependent endocytosis, internalization 
occurs in caveolae, which are invaginations of the plasma membrane rich in the protein caveolin and in cholesterol and glycolipids. The clathrin-dependent pathway is the one most studied. However, RTKs are not assigned to any particular internalization pathway; several factors together determine which pathway will be used. For example, it has been reported that low EGF concentrations favour the clathrin-dependent endocytosis of EGFR, whereas high, but still physiologically relevant, concentrations of EGF primarily promote caveolin-dependent internalization (Aguilar et al. 2005). Similar to EGFR, TrkA uses different internalization pathways. Although there is considerable evidence that NGF-TrkA complexes are internalized through a clathrin/dynamin-dependent process (Bilderback et al. 1999; Zweifel et al. 2005), TrkA has also been localized to caveola domains in PC12 cell lines upon NGF treatment (Peiro et al. 2000). In addition, TrkA can also be internalized by the clathrin-caveolin-independent pathways. Shao et al. identified an NGF-induced protein, Pincher (pinocytic chaperone) that mediates the endocytosis and trafficking of NGF and TrkA through macropinacytosis (Shao et al. 2002). They found that in PC12 cells, the overexpression of Pincher dramatically stimulated the NGF-induced endocytosis of TrkA in response to NGF, unexpectedly at sites of clathrin-independent macropinocytosis within cell surface ruffles.

Ubiquitination has been implicated in the process of endocytosis. In yeast, monoubiquitination has been found to be sufficient as an endocytic internalization signal (Terrell et al. 1998) and K63 poly-ubiquitination facilitates endocytosis (Galan et al. 1997). However, in eukaryotic cells this situation is more complicated. Sigismund et al. reported that when EGFR is stimulated with low doses of EGF it is internalized without ubiquitination almost exclusively through the clathrin-dependent pathway but that with higher concentrations of ligand a substantial fraction of the receptor is endocytosed through a clathrin-independent, lipid-raft-dependent route as the receptor becomes ubiquitinated (Sigismund et al. 2005). Regarding the NGF receptor TrkA, Geetha et al. found the K63 polyubiquitination is required for TrkA internalization and signalling (Geetha et al. 2005), while Arévalo et al. revealed that the multi-monoubiquitination of TrkA by Nedd4-2 does not affect TrkA internalization upon NGF treatment (Arevalo et al. 2006b). 
Therefore, which endocytic pathway a receptor will choose relies on many factors, such as the cell type expressing the receptor, the ligand concentration and its level and type of ubiquitination.

\subsubsection{Factors that modulate receptor trafficking}

Intracellular trafficking is a complicated and well-organized process. During this process many proteins and complexes are involved.

\section{ESCRT}

Ligand binding induces the activation and ubiquitination of the receptors and, consequently, their endocytosis to the EE. After the internalized cargoes have reached the EE, the Endosomal Sorting Complex for Transport (ESCRT) machinery recognizes the ubiquitinated cargoes and prevents their recycling and retrograde trafficking (Raiborg et al. 2009). The ESCRT machinery consists of ESCRT-0, ESCRT-I, ESCRT-II and ESCRT-III plus several accessory components. The ESCRT-0 complex comprises two subunits, Hrs and STAM, which localize to endosomal membranes. Both can engage ubiquitinated substrates destined for lysosomal degradation (Mayers et al. 2011). ESCRT-0 has been shown to form domains of clustered cargo but does not deform membranes. In combination, ESCRT-I and ESCRT-II deform the membrane into buds, in which the cargo is confined (Wollert et al. 2010). Following binding with ESCRT-I, ESCRT-II and ESCRT-III, the EEs carrying the internalized cargoes mature to LEs and then fuse with lysosomes for degradation (Grabbe et al. 2011).

\section{DUBs}

As is known, endocytic cargoes can escape from entering the degradation pathway by being transported to the RE and returned to the cell surface. If the ESCRT machinery is in charge of the degradation pathway there must be other modulators involved in the recycling pathway. The ubiquitination of RTKs initiated at the plasma membrane is regarded as the degradation tag for its substrate. However, this is a reversible process. It has been shown that at the EE, deubiquitination of the internalized cargoes caused by 
deubiquitinating enzymes (DUBs) redirects the sorting cargoes to recycling. The role of DUBs in the sorting process has been illustrated by two structurally unrelated endosomal DUBs, AMSH and UBPY, in mammalian cells (McCullough et al. 2006; Row et al. 2006). They are recruited not only by ESCRT-III and Alix but also by the ESCRT-0 subunit STAM to endosomes in order to remove polyubiquitin chains from substrates. Interestingly, UBPY and AMSH have differential substrate preferences. Although both deubiquitinate Lys-63-linked polyubiquitin chains, UBPY, but not AMSH, also uses Lys-48-linked polyubiquitin chains as a substrate (Row et al. 2006; Raiborg et al. 2009), which raises the possibility that these DUBs might function in the remodelling of ubiquitin chains attached to the cargo.

\subsubsection{Intracelluar trafficking of TrkA}

The intracellular trafficking of RTKs plays an important role in their biological functions. Upon NGF treatment, TrkA is internalized to EE regardless of the mode of entry, where the decision is made to be transported either to LE and lysosomes for degradation, or to $\mathrm{RE}$ for reinsertion into the plasma membrane (Figure Intr7). It has been reported that TrkA recycles back to the cell surface in a ligand-dependent manner in both neurosecretory cells and neurons. Conversely, TrkB are predominantly sorted to the degradative pathway (Sommerfeld et al. 2000; Chen et al. 2005). Compared with TrkB, TrkA produces a prolonged activation of $\mathrm{PI} 3 \mathrm{~K} / \mathrm{Akt}$ signalling as well as survival responses upon prolonged neurotrophin treatment (Chen et al. 2005). Therefore, different endocytic trafficking pathways lead to distinct biological properties.

The identification of specific markers of each endosome facilitates the investigation of the intracellular trafficking of internalized receptors. It has been reported that Rab proteins -small GTP-binding proteins of the Ras superfamily- reside in a particular type of endosome and function by recruiting specific effector proteins (Simons et al. 1993; Novick et al. 1997; Armstrong 2000). For example, Rab5 is a marker for EE, while Rab7 is one for LE and Rab11 is a marker for RE (Sorkin et al. 2009). 


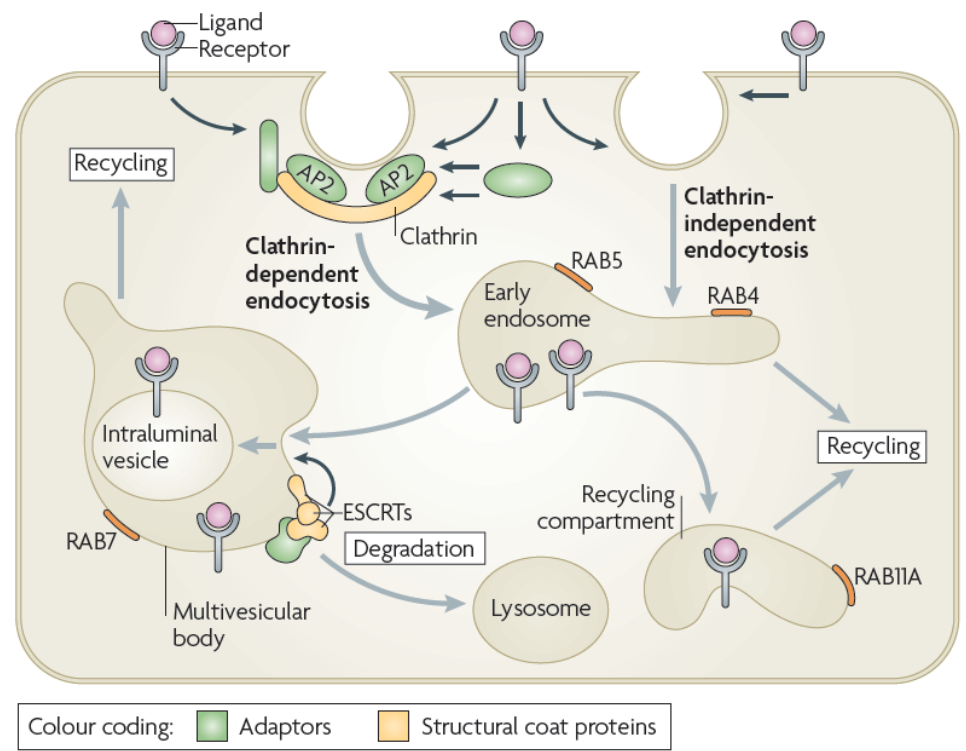

Figure Intr7. Pathways of receptor intracellular trafficking (Sorkin et al. 2009)

Endocytic vesicles derived from both clathrin-dependent and clathrin-independent endocytosis fuse with early endosomes. Following their internalization into early RAB5-containing endosomes, receptors can rapidly recycle back to the plasma membrane, traffic to the recycling compartment that contains RAB11A, or remain in endosomes, which mature into multivesicular bodies (MVBs) and late endosomes. Fusion of late endosomes and MVBs with lysosomes carrying proteolytic enzymes results in cargo degradation.

\subsubsection{Retrograde transport of TrkA}

TrkA is expressed in small DRG neurons that are characterized by the long distance travelled by their axons to reach the tissue they innervate. Therefore, NGF-TrkA signalling triggered in the distal axon innervating the target tissue must travel retrogradely for a considerable distance to the cell body to modulate gene expression (Howe et al. 2001; Delcroix et al. 2003; Zweifel et al. 2005). Early work studying the retrograde transport of ${ }^{125}$ I-NGF showed that NGF is retrogradely transported in sympathetic and sensory neurons (Claude et al. 1982). Neurons were grown in compartmentalized culture dishes and their distal processes were exposed to ${ }^{125}$ I-NGF. After 8 hours of transport, the ${ }^{125}$ I-NGF label was primarily localized to the MVB and the lysosomes when the radioactivity in the cell bodies had reached steady-state. More recently, phosphorylated TrkA (pTrkA) has also been shown to be transported retrogradely, together with neurotrophins, in neurons in vivo in a manner that is dependent on endogenous target-derived NGF, (Tsui-Pierchala et al. 1999). However, it is possible that neurotrophin is not necessary in retrograde endosomes (Maclnnis et al. 2002). It is known that a high 
concentration of Trk neurotrophin receptors, as may be found in Trk-containing vesicles, can support receptor autophosphorylation and signalling regardless of the ligand, at least in PC12 cells (Hempstead et al. 1994). Furthermore, neurotrophins, Trks, and activated components of the Ras-MAP kinase signalling pathway have also been found in retrograde endosomes (Howe et al. 2001).

Because the passive diffusion of signalling effectors is far too slow to account for such retrograde signalling, there must be an active transport pathway or transport machinery responsible for this. Since it is known that TrkA undergoes intracellular trafficking in the EE and LE, in which its activity is maintained, it has been proposed that signalling endosomes carrying TrkA activity are those due to undergo retrograde transport (Grimes et al. 1996; Philippidou et al. 2011) (Figure Intr8). However, the nature and component characteristics of signalling endosomes are not completely understood. In 2003, Delcroix et al. provided evidence that TrkA signalling endosomes showed the characteristics of EEs (Delcroix et al. 2003), although it has also been reported that Rab7, which is present in LEs, is a key component in axonal retrograde transport by controlling a vesicular compartment involved in neurotrophin traffic (Deinhardt et al. 2006). In 2011, Philippidou et al. found that retrogradely transported Trk was delivered to the soma in multivesicular bodies (MVB), which are derived from Pincher- and Rac-dependent macropinocytosis. They further found that retrograde signalling through Pincher-generated Trk-multivesicular bodies was distinctively refractory to signal termination by lysosomal processing, resulting in sustained somal signalling and neuronal gene expression (Philippidou et al. 2011). It seems that apart from the characteristics of endosomes, retrograde signalling endosomes must possess their own distinguishing feature to avoid being degraded before they are transported retrogradely to the cell body. Recently, the role of actin depolymerization in this process has been addressed. It is known that both NGF and NT3 bind to TrkA in a target-derived manner in sympathetic neurons, but only NGF triggers the formation of TrkA-containing signalling endosomes that are transported in a retrograde manner to the somata to promote neuronal survival. Upon comparing the different routes of activated TrkA in response to NGF and NT3, Harrington et al. found that actin depolymerization was 
essential for the initiation of NGF/TrkA endosome trafficking and that the actin filament-severing protein cofilin colocalized to a greater extent with TrkA endosomes in NGF-treated neurons than in those treated with NT3 (Harrington et al. 2011). Moreover, they observed an impaired retrograde transport of TrkA endosomes in NGF-treated neurons when shRNA against cofilin was expressed. In addition, they found that the acidic condition was involved in the retrograde transport of TrkA. NGF can promote a survival signal because, unlike NT3, it forms stable complexes with TrkA under acidic conditions, a property that enables this ligand to trigger TrkA-mediated signalling in endosomes, the recruitment of actin-organizing proteins, and the retrograde transport of TrkA endosomes.

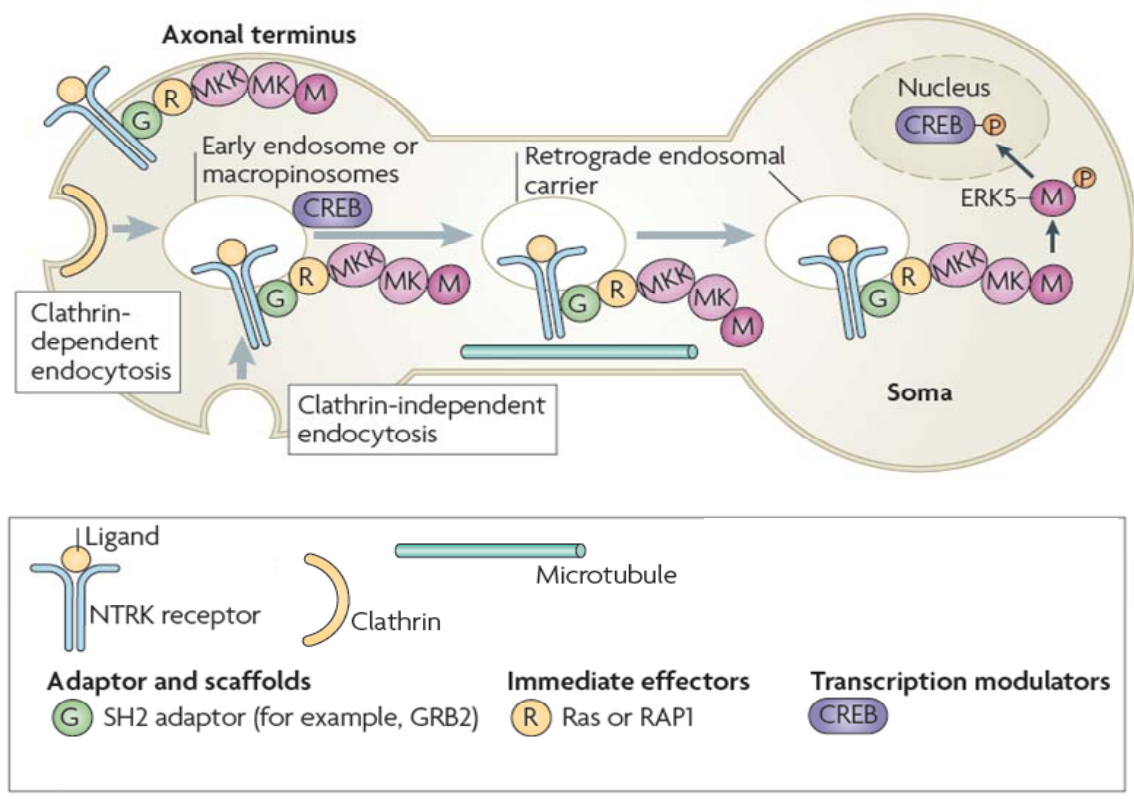

\section{Figure Intr8. Signalling processes that begin at the cell surface and continue in endosomes (Sorkin et al. 2009).}

Ligand-bound nerve growth factor receptors (NTRKs) are internalized by clathrin-dependent and clathrin-independent endocytosis, with associated components of the MAPK signalling cascade, into early endosomes or macropinosomes that have a multivesicular body-like morphology. NTRK signalling complexes are delivered to the soma in retrograde endosomal carriers, which are a population of early or late endosomes and macropinosomes, by dynein motor-mediated microtubular transport. The MAPK extracellular signal-regulated kinase 5 (ERK5) is then phosphorylated and activated in the soma. It phosphorylates the cAMP responsive element-binding protein (CREB), which regulates the transcription of anti-apoptotic genes. 
In sum, through retrograde transport the NGF synthesized and released by target tissues promotes the survival of TrkA-expressing sensory neurons, which are located in the DRGs. Upon analyzing the signalling pathways triggered by retrogradely transported TrkA, it has been found that retrogradely transported TrkA induces the activation of ERK5 and the phosphorylation of CREB in the neuronal soma to maintain cell survival (Figure Intr8) (Kuruvilla et al. 2000; Watson et al. 2001). At the same time, signalling proteins of the Rap1/Erk1/2, p38MAPK, and PI3K/Akt pathways have also been identified in the retrograde endosomes in sensory neurons, revealing the complexity and the importance of the retrograde transport of TrkA in its biological functions (Delcroix et al. 2003).

\subsection{Pain sensation and TrkA-expressing DRG neurons}

According to the International Association for the Study of Pain the definition of pain states that, "Pain is an unpleasant sensory and emotional experience associated with actual or potential tissue damage, or described in terms of such damage". Pain is primarily detected by the nerve terminals of sensory neurons, whose cell bodies are located in the DRG and TG. Sensory neurons are pseudo-unipolar neurons with two branches from one axon that are extended and targeted in two directions: to the periphery and to the spinal cord. Depending on their type, sensory neurons project to different laminae of the spinal cord (Figure Intr9). After synaptic transmission and modulation inside the primary sensory neuron and spinal cord, nociceptive signals reach the brain, where they are finally perceived as sensations of touch, warmth, cold, all kinds of pain, limb movements and limb spatial positions within the context of cognitive and environmental factors (Woolf 2000). 


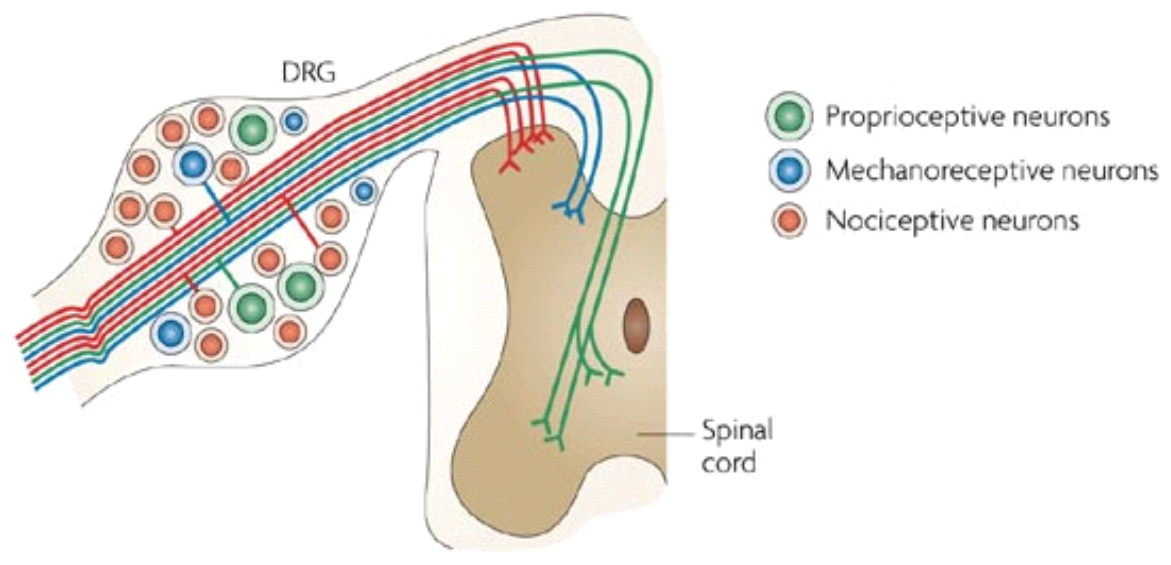

Nature Reviews | Neuroscience

Figure Intr9. Subclasses of dorsal root ganglia neurons and sensory input to the spinal cord (Marmigere et al. 2007)

Different groups of sensory neurons in DRGs project to different lamini of the spinal cord. nociceptive neurons terminating in the dorsal horn, mechanoreceptors in deeper laminae of the dorsal spinal cord, proprioceptive neurons in the intermediate zone and in the ventral spinal cord horn.

Sensory neurons located in the DRGs or TGs are not homogenous. For example, in DRGs they are classified in different groups: (1) based on morphology, sensory neurons can be divided into small-, medium- and large-diameter neurons; (2) according to their axonal fibres, these neurons have been grouped into unmyelinated $C$ fibre, thinly myelinated $A \delta$ fibre, myelinated $A \beta$ fibre and $A \alpha$ fibre neurons; (3) according to their survival-dependence on the corresponding neurotrophins, sensory neurons in the DRGs are divided into TrkA-, TrkB- and TrkC-expressing neurons; (4) they can also be distinguished from each other by whether they express neuropeptides or not, and which type (Table Intr1). There is an overlap among the different classifications; as can be seen in Table Intr1, small diameter neurons contain thinly myelinated A $\delta$ fibre neurons and unmyelinated $\mathrm{C}$ fibre neurons, which can be further classified by the receptor they express and the neuropeptide they release. Aठ fibre neurons can be divided into type I and type II, depending on whether they express TrkA or neuropeptide. (Table Intr1). 
Table Intr1: Classification of sensory neurons from DRG

\begin{tabular}{|c|c|c|c|}
\hline Size (diameter) & Fibres & Sub-groups & Characteristics \\
\hline \multirow{4}{*}{$\begin{array}{c}\text { Small } \\
\text { neurons }\end{array}$} & \multirow{2}{*}{$\begin{array}{c}\text { Aठ fibres } \\
\text { (Thiny-myelinated) }\end{array}$} & Type I & $\begin{array}{l}\text { - Respond to mechanical stimuli } \\
\text { - Respond to chemical stimuli } \\
\text { - have relatively high heat thresholds } \\
\text { (>50 } \mathrm{C} \text { ) (Basbaum et al. 2009). }\end{array}$ \\
\hline & & $\begin{array}{c}\text { Type II } \\
\text { (TrkA+ peptidergic) }\end{array}$ & $\begin{array}{l}\text { - A very high mechanical threshold. } \\
\text { - A much lower heat threshold. } \\
\text { - Activity of this afferent almost } \\
\text { certainly mediates the "first" acute } \\
\text { pain (Basbaum et al. 2009) }\end{array}$ \\
\hline & \multirow[t]{2}{*}{$\begin{array}{c}\text { C fibres } \\
\text { (Unmyelinated) }\end{array}$} & $\begin{array}{l}\text { Peptidergic: } \\
\qquad \text { SP+ } \\
\text { CGRP+ } \\
\text { TrkA+, } \\
\text { (Sah et al. 2003) }\end{array}$ & $\begin{array}{l}\text { Polymodal: } \\
\text { - thermal } \\
\text { - mechanical } \\
\text { - chemical }\end{array}$ \\
\hline & & $\begin{array}{l}\text { non-peptidergic: } \\
\text { CGRP-, TrkA -, c-Ret+, } \\
\text { Mrg+, P2X3+ } \\
\text { (Sah et al. 2003) }\end{array}$ & $\begin{array}{l}\text { A population of such neurons has } \\
\text { been reported as mechanically } \\
\text { insensitive (Perl 2007) }\end{array}$ \\
\hline $\begin{array}{l}\text { medium-sized } \\
\text { neurons }\end{array}$ & $A \beta$ fibres & $\begin{array}{l}\text { TrkB+ and/or } \\
\text { TrkC+ neurons } \\
\text { (de Carlos et al. 2006) }\end{array}$ & $\begin{array}{l}\text { Slight touch, pressure and vibration } \\
\text { low threshold mechanoreceptive } \\
\text { neurons (Djouhri et al. 2004) }\end{array}$ \\
\hline Large neurons & Aa fibres & $\begin{array}{l}\text { TrkC+ neurons } \\
\text { (Matsuo et al. 2000) }\end{array}$ & $\begin{array}{l}\text { proprioceptive neurons (Davies } \\
\text { 1986; Pecho-Vrieseling et al. 2009) }\end{array}$ \\
\hline
\end{tabular}

The role of NGF/TrkA in nociception has been widely addressed: (1) NGF levels were found to be elevated in peripheral neuropathic pain (Herzberg et al. 1997; Li et al. 2003) and direct administration of NGF into the sciatic nerve was seen to produce hyperalgesia (Ruiz et al. 2004); (2) TrkA-expressing neurons are mainly involved in pain sensation (Table Intr1), while TrkB- and TrkC-expressing sensory neurons in the DRGs are mainly in charge of physiological sensations (Marmigere et al. 2007); (3) TrkA and NGF knock-out mice show extensive losses of DRG neurons and fail to respond to painful stimuli (Crowley et al. 1994; Smeyne et al. 1994); (4) elevated NGF levels have been found in peripheral neuropathic pain (Herzberg et al. 1997; Li et al. 2003) and direct administration of NGF into the sciatic nerve produces hyperalgesia (Ruiz et al. 2004); (5) TrkA is the 
gene responsible for congenital insensitivity to pain with anhidrosis (CIPA) (Indo et al. 1996), a hereditary autosomal recessive disorder characterized by recurrent episodic fever, anhidrosis (inability to sweat), the absence of reaction to noxious stimuli, self-mutilating behaviour, and mental retardation. TrkA mutations have been identified in an extracellular domain involved in NGF binding, as well as in the intracellular signal transduction domain in CIPA patients (Indo 2001; Indo 2010). In light of the above, NGF/TrkA can be said to play a very important role in pain sensation. Any alteration or mutation in TrkA may cause disorders or functional changes in nociception.

How does NGF/TrkA regulate pain sensation? NGF/TrkA signalling can modulate pain sensation in several ways. (1) Through regulating TRPV1. TRPV1 belongs to the transient receptor potential channel family (TRP) and has been well studied and its important role in pain sensation has been demonstrated. It has been reported that the use of a chemical method to ablate neurons expressing TRPV1 nullifies heat pain (Mishra et al. 2010). Interestingly, TRPV1 has been shown to be regulated by NGF/TrkA signalling. TRPV1 expression was found to be increased within TrkA-positive neurons after inflammation (Amaya et al. 2004), and upon NGF binding to TrkA the PI3 kinase pathway-Src kinase binds to and phosphorylates TRPV1 for insertion into the surface membrane (Zhang et al. 2005). This explains most of the rapid sensitizing actions of NGF. (2) Through modulating pain-related neuropeptides such as substance $\mathrm{P}(\mathrm{SP})$ and calcitonin gene-related peptide (CGRP). SP was first discovered in 1931 as a tissue extract that caused intestinal contraction in vitro (US et al. 1931). With further investigation, SP was found to be a very important player in pain sensation, especially in carrying the pain signal to the brain (Harmar et al. 1980; Hua et al. 1999; McLeod et al. 1999). The fact that activation of TrkA by NGF in neurons leads to the generation and release of SP suggests a role of TrkA in nociception (Lindsay et al. 1989). The release of CGRP in the dorsal spinal cord has also been associated with nociceptive transmission, while its release from perivascular nerve endings causes neurogenic vasodilatation (Rosenfeld et al. 1983; Benemei et al. 2009). Double labelling using markers for several DRG subpopulations revealed that nearly all (92\%) TrkA-immunoreactive cells express CGRP (Averill et al. 1995). (3) Through other 
modulators of pain. For example, BDNF is synthesized in the primary sensory neurons and is involved in the modulation of painful stimuli (Obata et al. 2006). It has also been found to be up-regulated in DRG through an NGF-dependent mechanism (Michael et al. 1997).

The crucial role NGF/TrkA in nociception sheds light on its application in pain relief. As is known, the widely used non-steroidal anti-inflammatory drugs (NSAIDs) have side effects of gastrointestinal and renal morbidity, while dependence on and addiction to opioids for severe pain limit their use. Therefore, new drugs designed specifically to treat chronic pain are urgently needed to overcome the drawbacks of currently available compounds. In this sense, drugs targeting the NGF/TrkA pathway may be good candidates. To date, antibodies against TrkA and NGF have been developed and applied for the treatment of pain (McMahon et al. 1995; Koltzenburg et al. 1999; Halvorson et al. 2005). Application of the TrkA monoclonal antibody MNAC13 shows potent analgesic effects in inflammatory and chronic pain (Ugolini et al. 2007) and in reducing fracture-related pain, with no evident effect on the density or function of sensory and sympathetic nerve fibres (Ghilardi et al. 2011). The humanized monoclonal antibody against NGF, Tanezumab, has shown equal or greater efficiency than opiates or NSAIDs in preclinical models of pain. Phase I and phase II clinical trials of Tanezumab in osteoarthritis, chronic low back pain and interstitial cystitis have demonstrated the drug's analgesic efficacy across these three chronic pain settings (Cattaneo 2010; Lane et al. 2010; Katz et al. 2011). However, Tanezumab's steady progress faltered when some individuals in phase III osteoarthritis trials developed joint damage (Garber 2011). These side effects suggest that more work needs to be done to address the mechanisms of NGF-TrkA-mediated nociception to provide better insight into how chronic pain can be tackled. 
Introduction 
2 AIMS 
As a continuation of the study of Arevalo et al. on Nedd4-2 (Arevalo et al. 2006b), and based on the published data related to NGF-TrkA signalling, ubiquitination and biological functions, the Aims of this thesis are the following:

- To test the role of TrkA ubiquitination mediated by Nedd4-2 in the turnover and intracellular trafficking of TrkA upon NGF treatment in DRG sensory neurons.

- To assess the role of TrkA ubiquitination mediated by Nedd4-2 in TrkA functions in vitro and in vivo. 
3 Materials and Methods 


\subsection{Materials}

\subsubsection{General reagents}

Table M1. General reagents

\begin{tabular}{|c|c|c|}
\hline Names & Application in the project & Concentration \\
\hline MEM & Cell culture media & -- \\
\hline NeuroBasal & Cell culture media for neurons & -- \\
\hline L-15 & Tissue dissection & -- \\
\hline FBS & Cell culture supplement & $10 \%$ \\
\hline Glucose & Cell culture supplement & $0.4 \%$ \\
\hline Glutamine & Cell culture supplement & $2 \mathrm{mM}$ \\
\hline NGF & Nerve growth factor & $50 \mathrm{ng} / \mathrm{ml}$ \\
\hline BDNF & Brain-derived nerve factor & $25 \mathrm{ng} / \mathrm{ml}$ \\
\hline B27 / NS21 & Neuron culture supplement & $1: 100$ \\
\hline Pen/Strep & Cell culture & $100 \mathrm{U} / \mathrm{ml}$ \\
\hline Trypsin & Tissue dissection & $0.25 \%$ \\
\hline 5-Fluoro Uridine & Inhibitor to kill dividing cells & $2.44 \mu \mathrm{g} / \mathrm{ml}$ \\
\hline Uridine & Cell culture & $2.44 \mu \mathrm{g} / \mathrm{ml}$ \\
\hline Matrigel & Cell substrate & $1: 10$ \\
\hline Poly-D-Lysine (PDL) & Cell substrate & $0.1-1 \mu \mathrm{g} / \mathrm{ml}$ \\
\hline SDS & Protein lysis & $0.2 \%$ \\
\hline Sodium orthovanadate & Inhibitor of all tyrosine protein phosphatases & $1 \mathrm{mM}$ \\
\hline Sodium fluoride (NaF) & Inhibitor of serine/threonine protein phosphatase & $10 \mathrm{mM}$ \\
\hline Aprotinin & Protease inhibitor & $1 \mu \mathrm{g} / \mathrm{ml}$ \\
\hline Leupeptine & Lysosomal protease inhibitor & $2 \mu \mathrm{g} / \mathrm{ml}$ \\
\hline$\beta$-glycerophosphate & Reversible inhibitor of aspartic proteases & $20 \mathrm{mM}$ \\
\hline PMSF & Protease inhibitor & $1 \mathrm{mM}$ \\
\hline
\end{tabular}


Table M1 (continued). General reagents

\begin{tabular}{|c|c|c|}
\hline Names & Application in the project & Concentration \\
\hline BSA & Protein concentration measurement & $1 \mu \mathrm{g} / \mu \mathrm{l}$ \\
\hline Sulfo-NHS-SS-Biotin & Surface protein labeling & $0.5 \mu \mathrm{g} / \mathrm{ml}$ \\
\hline NeutroAvidin-Beads & Biotin pulldown & -- \\
\hline DTT & Stripping biotin from labeled protein & $50 \mathrm{mM}$ \\
\hline Z-VAD-FMK & Inhibitor of apoptosis & $20 \mu \mathrm{M}$ \\
\hline Cycloheximide & Inhibitor of protein translation & $0.1 \mathrm{mg} / \mathrm{ml}$ \\
\hline Trizol & RNA extraction & -- \\
\hline Chloroform & RNA extraction & -- \\
\hline Isopropyl alcohol & RNA extraction & -- \\
\hline GoScriptTM Reverse Transcript Kit & Reverse Transcript & -- \\
\hline Taq DNA polymerase & PCR & -- \\
\hline 2X Master Mix & qPCR & -- \\
\hline Lipofectamine 2000 & Transfection reagent & -- \\
\hline Opti-MEM & Transfection reagent & -- \\
\hline Tween 20 & Cell permeabilization & $0.1 \%$ \\
\hline Triton $\mathrm{X}-100$ & Cell permeabilization & $0.1 \%$ \\
\hline Hoechst 33342 & Staining nulei & $1: 10,000$ \\
\hline Cresyl violet & Staining neurons & $0.5 \%$ \\
\hline Proteinase $\mathrm{K}$ & Tissue digestion & $20 \mu \mathrm{g} / \mathrm{ml}$ \\
\hline PFA & Tissue fixation & $4 \%$ \\
\hline Formalin & Formalin assay & $5 \%$ \\
\hline RNAse A & In situ hybridization & $20 \mu \mathrm{g} / \mathrm{ml}$ \\
\hline Normal Goat Serum & Inmmunofluorescece / In situ hybridization & $10 \%$ \\
\hline Anti-DIG-AP Ab & In situ hybridization & $1: 3000$ \\
\hline AP substrate & In situ hybridization & -- \\
\hline tetramysole & In situ hybridization & $1 \mathrm{mM}$ \\
\hline EDTA & In situ hybridization & $2 \mathrm{mM}$ \\
\hline
\end{tabular}




\subsubsection{Buffers}

- $2 \times$ HBS Buffer

$140 \mathrm{mM} \mathrm{NaCl}, 1.5 \mathrm{mM}$ Na2HPO4, 50 mM HEPES, pH 7.05

- NP-40 Lysis Buffer

$20 \mathrm{mM}$ Tris- $\mathrm{HCl} \mathrm{pH} \mathrm{8,} 137 \mathrm{mM} \mathrm{NaCl}, 10 \%$ glycerol, 1\% Nonidet P-40, 2 mM EDTA with protease and phosphatase inhibitors (Sodium orthavanadate, $\mathrm{NaF}$, Aprotinin, Leupeptin, $\beta$-glycerophosphate and PMSF).

- $2 \times$ SDS Loading Buffer

$100 \mathrm{mM}$ Tris $\mathrm{pH} 7.5,25 \%$ glycerol, $2 \%$ SDS, $0.01 \%$ bromophenol blue

- Electrophoresis Buffer

25 mM Tris, pH8.3; 192 mM Glycine; 0.1\% SDS (1XTris/Glycine/SDS buffer)

- Western Blot Transfer Buffer

25 mM Tris, pH8.3; 192 mM Glycine; Metanol 10\%-20\% (1XTris/Glycine/Metanol buffer)

- TBST Buffer

$10 \mathrm{mM}$ Tris $\mathrm{pH}$ 8, $150 \mathrm{mM} \mathrm{NaCl}, 1 \mathrm{mM}$ EDTA, $0.1 \%$ Tween 20

- Blocking Buffer

$2 \%$ BSA in TBST (primary antibody) or $3 \%$ milk in TBST (secondary antibody)

- Enhanced chemiluminescent buffer (ECL)

Solution A (500 mL): $6.057 \mathrm{~g}$ Tris, $195 \mathrm{mg}$ luminol, $237 \mathrm{mg}$ P-iodophenol, pH 9.35.

Solution $\mathrm{B}: 37 \% \mathrm{H}_{2} \mathrm{O}_{2}$.

( $10 \mathrm{ml}$ solution $\mathrm{A}$ and $1 \mu \mathrm{l}$ solution $\mathrm{B}$ were mixed and added onto blots for 1 minute).

- Membrane-stripping solution

1. Acidic glycine solution (25 mM glycine- $\mathrm{HCl}, \mathrm{pH} 2.5$ )

2. $1 \%$ (w/v) SDS in $\mathrm{H}_{2} \mathrm{O}$

- Biotinylation Buffer

PBS containing $1 \mathrm{mM} \mathrm{CaCl}_{2}, 0.5 \mathrm{mM} \mathrm{MgCl}_{2}$ and $0.5 \mathrm{mg} / \mathrm{ml}$ Biotin

- Homogenization Buffer

$250 \mathrm{mM}$ Sucrose, $20 \mathrm{mM}$ Tricine, $1 \mathrm{mM}$ EDTA, $2 \mathrm{mM} \mathrm{MgCl}_{2}$, $\mathrm{pH} 7.8$ 
- In situ hybridization Acetylation Buffer

$1.3 \mathrm{ml}$ of Triethanolamine (Sigma), $260 \mu \mathrm{l}$ of $12 \mathrm{~N} \mathrm{HCl}, 250 \mu \mathrm{l}$ Acetic anhydride (Fluka), were mixed and reach to a final volume of $100 \mathrm{ml}$ by DEPC treated $\mathrm{H}_{2} \mathrm{O}$.

- In situ hybridization Hybridization Buffer

$50 \mathrm{ml}$ of redistilled formamide (Sigma), $20 \mathrm{ml}$ of $50 \%$ stock dextran sulfate (Sigma), 2 $\mathrm{ml}$ of $50 \times$ Denhardt's (Sigma), $2.5 \mathrm{ml}$ of $10 \mathrm{mg} / \mathrm{ml}$ stock yeast tRNA (Sigma), $6 \mathrm{ml}$ of 5M $\mathrm{NaCl}, 2 \mathrm{ml}$ of $1 \mathrm{M}$ Tris- $\mathrm{HCl}$ (pH8.0), $1 \mathrm{ml}$ of $0.5 \mathrm{M}$ EDTA, $1 \mathrm{ml}$ of $1 \mathrm{M} \mathrm{Na}_{3} \mathrm{PO}_{4}, 5 \mathrm{ml}$ of $20 \%$ Sarcosyl (Sigma) and $10.5 \mathrm{ml}$ of $\mathrm{dH}_{2} \mathrm{O}$ were mixed together to obtain a $100 \mathrm{ml}$ solution.

- In situ hybridization RNAse Buffer.

$880 \mathrm{ml}$ of $\mathrm{dH}_{2} \mathrm{O}, 100 \mathrm{ml}$ of $5 \mathrm{M} \mathrm{NaCl}, 10 \mathrm{ml}$ of Tris- $\mathrm{HCl}(\mathrm{pH} 7.5), 10 \mathrm{ml}$ of $0.5 \mathrm{M}$ EDTA were mixed together to obtain a1-L solution.

- In situ hybridization AP Stain Buffer.

To make $100 \mathrm{ml}: 2 \mathrm{ml}$ of $5 \mathrm{M} \mathrm{NaCl}, 10 \mathrm{ml}$ of $1 \mathrm{M}$ Tris- $\mathrm{HCl}$ (pH 9.5), $5 \mathrm{ml}$ of $1 \mathrm{M} \mathrm{MgCl}_{2}$, $0.1 \mathrm{ml}$ of Tween-20, and $82.9 \mathrm{ml}$ of $\mathrm{dH}_{2} \mathrm{O}$ were mixed together to obtain a $100-\mathrm{ml}$ solution. 


\subsubsection{Antibodies}

Table M2. Antibodies

\begin{tabular}{|c|c|c|c|c|}
\hline Name & Host & Company / Article & Use & MW (kDa) \\
\hline Nedd4-2 & Rabbit & (Arevalo et al. 2006b) & WB $1 \mu \mathrm{g} / \mathrm{ml}$ & $120 / 140$ \\
\hline pPLC- $\gamma$ & Rabbit & Cell Signaling & WB 1:1000 & 155 \\
\hline pTrk (Y490) & Rabbit & Cell Signaling & WB 1:1000 & 140 \\
\hline pAkt (Ser473) & Mouse & Upstate & WB 1:1000 & 60 \\
\hline pMAPK & Mouse & Cell Signaling & WB 1:2000 & $42 / 44$ \\
\hline TrkA (C-14) & Rabbit & Santa Cruz & WB 1:400 & $110 / 140$ \\
\hline TrkA RTA & Rabbit & gift from L. Reichardt & WB 1:2000 & $110 / 140$ \\
\hline TrkA(C14)-agarose & Rabbit & Santa Cruz & IP $10 \mu \mathrm{l} / \mathrm{sam}$ & $110 / 140$ \\
\hline p75 (9992) & Rabbit & Gift from M. Chao & WB 1:2000 & 75 \\
\hline pTrkA (Y785F) & Rabbit & Gift from M. Chao & IF 1:500 & 140 \\
\hline Akt & Mouse & Santa Cruz & WB 1:200 & 60 \\
\hline MAPK & Rabbit & Cell Signaling & WB 1:1000 & $42 / 44$ \\
\hline P4D1 (ubiquitin) & Rabbit & Santa Cruz & WB 1:400 & -- \\
\hline Rab5 & Mouse & BD & IF $1: 50$ & 25 \\
\hline EEA1 & Mouse & $\mathrm{BD}$ & IF 1:250 & 180 \\
\hline Rab11 & Mouse & BD & IF $1: 50$ & 25 \\
\hline Rab7 & Mouse & Sigma & IF $1: 200$ & 25 \\
\hline BIII-Tubulin & Rabbit & Sigma & WB 1:10000 & 55 \\
\hline GFP & Mouse & Molecular Probes and Chemicon & WB 1:3000 & 27 \\
\hline c-fos & Rabbit & Oncogene Research Products & IH 1:7500 & -- \\
\hline Protein A-HRP & & Life Technologies & $1: 8000$ & -- \\
\hline Protein G-HRP & & Life Technologies & $1: 8000$ & -- \\
\hline Anti-mouse HRP & & Jackson Labs & $1: 5000$ & -- \\
\hline Anti-rabbit HRP & & Jackson Labs & $1: 8000$ & -- \\
\hline
\end{tabular}

BD: Beckton Dickinson; Sam: sample 


\subsection{Methods}

\subsubsection{Cell Line Culture}

HEK293 and HEK293FT cell lines were cultured in DMEM media supplemented with 10\% Bovine Serum, $2 \mathrm{mM}$ glutamine and $100 \mathrm{U} / \mathrm{ml}$ Pen/Strep and maintained in a humidified incubator at $37^{\circ} \mathrm{C}$ with $5 \% \mathrm{CO}_{2}$.

\subsubsection{HEK293 cell transfection using Calcium Phosphate}

HEK293 cells $\left(3 \times 10^{6}\right)$ were plated in a $10-\mathrm{cm}$ plate coated with PDL $(0.1 \mathrm{mg} / \mathrm{ml})$ on the day before transfection. The following day, the medium was replaced with fresh medium 2-3 hours before transfection. As mentioned in Table M3, solution A for transfection was prepared by mixing $10 \mu \mathrm{g}$ of plasmid and $150 \mu \mathrm{l}$ of $1 \mathrm{M} \mathrm{CaCl}_{2}$ together to a final volume of $500 \mu \mathrm{l}$ in a sterile 1.5-ml Eppendorf tube. The same amount of Solution B $(2 \times \mathrm{HBS}, \mathrm{pH}$ 7.05) was added in a sterile $15-\mathrm{ml}$ tube. To form the transfection complex, Solution B was

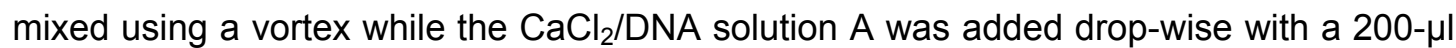
pipette. The mixture was incubated at room temperature for 20 min before distributing it over the HEK293 cells in the culture plate with gentle shaking to make the precipitate become evenly distributed. The cells were incubated for 16 hours and the medium was replaced by fresh medium to remove the $\mathrm{CaCl}_{2} / \mathrm{DNA} / \mathrm{HBS}$ transfection complexes. The cells were incubated for a further 1-2 days and transfection was confirmed for GFP expression under a fluorescence microscope.

Table M3. HEK 293 cell transfection using Calcium Phosphate

\begin{tabular}{cccc}
\hline Solution A & Quantity & Solution B & Quantity \\
\hline Plasmids & $10 \mu \mathrm{g}$ & $2 \times \mathrm{HBS}(\mathrm{pH} \mathrm{7.05)}$ & $500 \mu \mathrm{l}$ \\
$1 \mathrm{M} \mathrm{CaCl}_{2}$ & $150 \mu \mathrm{l}$ & & \\
$\mathrm{H}_{2} \mathrm{O}$ & Up to $500 \mu \mathrm{l}$ & & \\
\hline
\end{tabular}




\subsubsection{HEK293 cell transfection using Lipofectamine 2000}

One day before transfection, $4-8 \times 10^{5}$ cells were plated in each well of a 6-well plate with $1.5 \mathrm{ml}$ of complete growth medium without antibiotics to have the cells at $90-95 \%$ confluence at the time of transfection. $2 \mu \mathrm{g}$ of DNA and $6 \mu$ of Lipofectamine ${ }^{\mathrm{TM}} 2000$ were diluted in $50 \mu \mathrm{l}$ of Opti-MEM (or another medium without serum), respectively, mixed gently, and incubated for $5 \mathrm{~min}$ at room temperature; then, the diluted DNA was mixed with diluted Lipofectamine ${ }^{\mathrm{TM}} 2000$ and incubated for another $20 \mathrm{~min}$ at room temperature (the total volume was $100 \mu \mathrm{l}$ and the ratio of DNA $(\mu \mathrm{g})$ to Lipofectamine ${ }^{\mathrm{TM}} 2000(\mu \mathrm{l})$ was 1:3). When the plasmids did not express any fluorescent protein, a GFP-expressing vector was included in the DNA mixture to monitor transfection efficiency. After incubation, the DNA:Lipfectamine mixture was distributed to each well with cultured cells. The plate was shaken gently to make the DNA:Lipofectamine complex spread evenly and then replaced in the incubator at $37^{\circ} \mathrm{C}$ with $5 \%-95 \% \mathrm{CO}_{2}$-air. After 4-6 hours, the medium was changed with fresh complete medium to prevent toxicity to the cells. GFP expression in cells was used to test the transfection efficiency $24-48$ hours post transfection before performing any additional experiments.

\subsubsection{Lentivirus generation and infection}

The 5'-aagtggacaatttaggccgaa-3' sequence corresponding to nucleotides 727-747 of human Nedd4-2 cDNA was used to generate Nedd4-2 shRNA, which was subsequently cloned into the pLVTHM lentiviral vector. The Nedd4-2 sequence used to generate the shRNA has $100 \%$ homology with rat and mouse Nedd4-2 cDNAs. Control shRNA were generated using the 5'-gcgcgctttgtaggattcg-3' sequence from Euglena gracilis chloroplast DNA between $16 \mathrm{~S}$ and $16 \mathrm{~S}$ rRNA (Kuratomi et al. 2005).

Lentiviruses were generated by co-transfection of lentiviral vectors with helper plasmids into HEK293FT cells using the calcium phosphate method. Briefly, $2.5 \times 10^{6}$ HEK293FT cells were seeded on a $10-\mathrm{cm}$ plate the day before transfection. $9 \mu \mathrm{g}$ of pLVTHM control 
shRNA or pLVTHM-Nedd4-2 shRNA vector together with $6 \mu \mathrm{g}$ of psPAX2 and $5 \mu \mathrm{g}$ of pMD.2G plasmids were transfected into the cells. The medium was replaced with fresh medium without antibiotics after 16 hours of transfection to prevent damage to the cells that were later to become infected. 48 hours after transfection, the supernatants containing the lentivirus were collected, centrifuged at $3500 \mathrm{rpm}$ for $10 \mathrm{~min}$, filtered through a $0.45 \mu \mathrm{M}$ filter, and stored in aliquots at $-80^{\circ} \mathrm{C}$. To infect DRG neurons, $100 \mu \mathrm{l}$ of supernatant-containing lentivirus was added to $1 \times 10^{5}$ DRG cells cultured in the NB medium. Infected neurons were monitored by the expression of GFP. The efficiency of infection was also analyzed by western blot analysis, looking at Nedd4-2 protein depletion.

\subsubsection{Primary DRG Neuron Dissection and Culture}

DRGs were dissected at E13.5 and E15.5 from mouse and rat embryos, respectively. E0.5 was considered the day the plug was observed. The abdominal skin at midline and the muscles of the pregnant mother were cut to obtain the entire uterus, which was placed in a sterile $10-\mathrm{cm}$ tissue culture dish. Embryos were isolated from the uteri by cutting through the tissue surrounding the amniotic fluid, gently squeezed through an incision and placed in another sterile 10-cm dish containing L-15 media (Gibco). Under a dissecting microscope, the head, the tail and the ventral part (belly) of the embryos were removed to obtain the dorsal structures (back) containing the spinal cord. The entire spinal cord was exposed by carefully cutting between the vertebral column and the spinal canal at the rostral end using a micro-dissecting scissors, and the spinal cord was peeled out from the embryo with attached DRGs. Then, the DRGs were plucked off from spinal cord, pooled, and incubated with $0.25 \%$ trypsin (Gibco) in L-15 media for $45 \mathrm{~min}$ at $37^{\circ} \mathrm{C}$ followed by centrifugation to eliminate trypsin. The resulting cells were resuspended in plating medium (MEM, 10\% FBS, 0.4\% glucose, $2 \mathrm{mM}$ glutamine, $100 \mathrm{U} / \mathrm{ml}$ Pen/Strep). Single-cell suspensions were obtained by pipetting up and down 30 times with a 1-ml tip followed by 30 times with a 200- $\mu$ l tip. After counting, cells were plated in plating medium 
supplemented with the corresponding neurotrophin, NGF $(50 \mathrm{ng} / \mathrm{ml})$ or BDNF $(25 \mathrm{ng} / \mathrm{ml})$ overnight on plastic plates coated with Growth Factor Reduced Matrigel 1:10 diluted in MEM as substrate. On the following day, the media were changed to NB media (neurobasal media, B-27 or NS21, $0.4 \%$ glucose, $2 \mathrm{mM}$ glutamine), supplemented with NGF or BDNF, 5-fluorodeoxyuridine (5-FU) and uridine. Proliferating cells were killed after 3-4 days by $5-\mathrm{FU}$ and more than $95 \%$ of cells were neurons after 8 days.

\subsubsection{Western Blot}

\section{A) Sample preparation}

From cells in culture. To prepare samples from cell cultures, 100,000 DRG neurons cultured in 12-well plates were washed with cold PBS for the cells to detach themselves from the wells and after a spin to discard the supernatant the cells were lysed in $500 \mu \mathrm{l}$ of NP40 lysis buffer containing protease inhibitors by gentle shaking at $4^{\circ} \mathrm{C}$ for $40 \mathrm{~min}$. The samples were then centrifuged for $15 \mathrm{~min}$ at $12,000 \mathrm{rpm}$ at $4{ }^{\circ} \mathrm{C}$ and the supernatant containing the proteins was collected.

The Bradford Assay (BioRad) was used to quantify the concentration of the protein and 20 $\mu \mathrm{g}$ of lysates was mixed with $2 \times$ SDS Loading Buffer and boiled for $7 \mathrm{~min}$ to denature the proteins. Proteins were resolved using 6-12\% SDS-PAGE, transferred onto PVDF-membranes, and probed with antibodies against the different proteins. The remaining proteins were stored at $-20^{\circ} \mathrm{C}$ for later usage.

From tissue. To prepare samples from tissues, up to $5 \mathrm{mg}$ of fresh tissue from DRG, cortex or hippocampus was lysed in $500 \mu \mathrm{l}$ of NP-40 lysis buffer in the presence of $0.2 \%$ SDS and protease inhibitors at $4^{\circ} \mathrm{C}$. Samples were then homogenized by pipetting up and down to accelerate the lysis process and incubated at $4^{\circ} \mathrm{C}$ for 40 min with gentle shaking, followed by centrifuging for $15 \mathrm{~min}$ at $12,000 \mathrm{rpm}$ at $4{ }^{\circ} \mathrm{C}$. The supernatants containing proteins were transferred into a clean 1.5-ml Eppendorf tube and quantified for the 
concentration of protein using the Bradford Assay. For longer storage, prepared samples were stored at $-80^{\circ} \mathrm{C}$.

\section{B) Quantification of protein concentrations using the Bradford assay:}

Before quantification, a $5 \times$ Bradford Solution was diluted with $\mathrm{dd}_{2} \mathrm{O}$ to a $1 \times$ Bradford Solution (1/4 V/V). $0,2,5$ and $10 \mu \mathrm{g}$ of BSA were used to set up the standard curve of the assays by dissolving BSA in the buffer used to prepare the samples. Samples were mixed with $1 \mathrm{~mL}$ of $1 \times$ Bradford Solution and measured in a spectrophotometer with an absorbance wavelength of $595 \mathrm{~nm}$. The total protein concentration of each sample was obtained using linear regression fitting, when the $R^{2}$ values were more than 0.95 .

\section{C) Electrophoresis}

Depending on the molecular size of the proteins, different percentages of SDS-PAGE were prepared (e.g. $15 \mathrm{kDa}-12 \% ; 25 \mathrm{kDa}--10 \%$; $50 \mathrm{kDa}$ or higher--6\%), as shown in the table below (Table M4). Care was taken to avoid bubbles in the mixture during its pouring into the assembled supporters. Samples were loaded and run with electrophoresis buffer for $20 \mathrm{~min}$ at $80-100 \mathrm{~V}$ and then120-150 $\mathrm{V}$ for the remaining time until the front dye reached the bottom of the gel.

Table M4. Preparation of PAGE gels

\begin{tabular}{llllllll}
\hline \multicolumn{1}{c}{ Components } & \multicolumn{5}{c}{ Separating gel } & \multicolumn{2}{c}{ Stacking gel } \\
& $\mathbf{6 \%}$ & $\mathbf{7 . 5 \%}$ & $\mathbf{8} \%$ & $\mathbf{1 0 \%}$ & $\mathbf{1 2 \%}$ & $\mathbf{1 5 \%}$ & $\mathbf{4 . 0 \%}$ \\
\hline 30\% Acrylamide $(\mathrm{ml})$ & 2 & 2.5 & 2.7 & 3.3 & 4 & 5 & 0.52 \\
1.5M Tris, pH 8.8 +SDS $(\mathrm{ml})$ & 2.5 & 2.5 & 2.5 & 2.5 & 2.5 & 2.5 & $\mathrm{X}$ \\
\hline 0.5M Tris pH 6.8 +SDS $(\mathrm{ml})$ & $\mathrm{X}$ & $\mathrm{X}$ & $\mathrm{X}$ & $\mathrm{X}$ & $\mathrm{X}$ & $\mathrm{X}$ & 1 \\
\hline $\mathrm{H} 2 \mathrm{O}(\mathrm{ml})$ & 5.5 & 5 & 4.8 & 4.2 & 3.5 & 2.5 & 2.5 \\
$10 \%$ APS $(\mu \mathrm{l})$ & 100 & 100 & 100 & 100 & 100 & 100 & 40 \\
TEMED $(\mu \mathrm{l})$ & 10 & 10 & 10 & 10 & 10 & 10 & 4 \\
\hline
\end{tabular}




\section{D) Transfer of proteins onto a PVDF membrane}

The principle is that proteins with an electrical charge (provided by the SDS bound to them) can be induced to travel through a gel in an electrical field, such that the proteins can be transferred in an electrical field from the gel onto a sturdy support such as a membrane. Briefly, for wet transfer the gel and membrane were sandwiched between sponge and paper (sponge/paper/gel/membrane/paper/sponge) and all were clamped tightly together after ensuring no air bubbles were present between the gel and membrane. The sandwich was then submerged in transfer buffer to which an electrical field was applied $\left(400 \mathrm{~mA}, 4^{\circ} \mathrm{C}, 2\right.$ hours $)$. The negatively-charged proteins were transferred onto the membranes in the electrical field.

\section{E) Antibodies and detection}

Once the proteins had been transfered to the PVDF membrane, antibodies against the proteins we were interested in were used to blot the membrane. First, the membranes were blocked using blocking buffer for $20 \mathrm{~min}$ at room temperature or overnight at $4^{\circ} \mathrm{C}$ to prevent non-specific background binding of the primary and/or secondary antibodies to the membrane (which has a high capacity for binding proteins, and hence antibodies). Then, the primary antibody was diluted in the blocking buffer at the corresponding concentration and the membrane was incubated with shaking overnight at $4^{\circ} \mathrm{C}$. After washing $3 \times 10 \mathrm{~min}$ with TBST at room temperature, the blots were incubated with anti-rabbit or anti-mouse antibodies conjugated with HRP, depending on the source of the primary antibody. The secondary antibodies were diluted in TBST containing $3 \%$ non-fat milk and incubated for $40 \mathrm{~min}$ at room temperature with shaking. After $3 \times 10$ min washes with TBST the signals were detected with ECL buffer.

\section{F) Analysis of Western Blot results}

The results from Western blotting were scanned and analyzed with Image $\mathrm{J}$ for quantification. 


\section{G) Stripping and reprobing the membranes}

When it was necessary to re-blot the membrane, the antibodies were stripped from the membrane by incubating it with the acidic glycine solution $(25 \mathrm{mM}$ glycine- $\mathrm{HCl}, \mathrm{pH} 2.5)$ for $10 \mathrm{~min}$ at room temperature with shaking followed by incubation with $1 \%(\mathrm{w} / \mathrm{v})$ SDS for 10 min. After washing the blots three times for 5 min each with TBST, the blots were re-probed with new primary antibodies.

\subsubsection{Immunoprecipitation (IP)}

To detect specific modifications of certain proteins, it is necessary to separate or concentrate one protein from others; accordingly, immunoprecipitation (IP) is used. The principle of IP is based on the specificity of antibody-antigen binding and subsequent antibody-antigen pulldown with beads that recognize the antibody. Depending on whether the antibody is attached to the beads or not, the processes of IP may involve either one or two steps. In the case where the antibody was labeled directly with Sepharose beads, the total lysates were incubated with the antibodies at $4^{\circ} \mathrm{C}$ overnight with gentle shaking and the immune complexes were recovered by centrifugation and washed 3-5 times using lysis buffer. When the antibody was not labeled, the total lysates were incubated first with these antibodies overnight under gentle shaking, after which Sepharose protein A or G was added, depending on the species of the primary antibodies (Table M5) at $4^{\circ} \mathrm{C}$ for another 1-3 hours. After the incubation of protein A or $G$, the immune complexes were recovered by centrifugation and washed 3-5 times using lysis buffer. The immunoprecipitated proteins were eluted from the beads using $2 \times$ SDS loading buffer under heating, subjected to SDS-PAGE, and immunoblotted with the corresponding antibodies. To avoid problems with the IgG chains during the development of the signals, Protein-A or Protein-G conjugated HRP were used for the secondary antibodies when same species antibodies were used for both immunoprecipitation and western blotting. 
Table M5. Binding affinity of Protein A and Protein G

\begin{tabular}{|c|c|c|}
\hline Species Immunoglobulin Isotype & Protein A & Protein G \\
\hline Mouse $\operatorname{lgG} 1$ & + & +++ \\
\hline $\operatorname{lgG} 2 \mathrm{a}$ & +++ & +++ \\
\hline $\lg \mathrm{+} 2 \mathrm{~b}$ & ++ & ++ \\
\hline $\operatorname{lgG} 3$ & + & + \\
\hline $\lg \mathrm{+}$ & Use anti Mouse IgM \\
\hline All rabbit isotypes & +++ & ++ \\
\hline Rat $\operatorname{lgG} 1$ & - & + \\
\hline $\lg \mathrm{+} 2 \mathrm{a}$ & - & +++ \\
\hline $\lg \mathrm{+} 2 \mathrm{~b}$ & - & ++ \\
\hline $\operatorname{lgG} 2 \mathrm{c}$ & + & ++ \\
\hline
\end{tabular}

\subsubsection{Immunofluorescence (IF)}

The DRG neurons used to perform immunofluorescence (IF) analyses were cultured on coverslips coated with poly-D-Lysine (PDL) at $1 \mathrm{mg} / \mathrm{ml}$ and Growth Factor Reduced Matrigel with a low density to obtain separated cells. Where necessary, the attached DRGs were infected with $10 \mu \mathrm{l}$ of supernatants containing lentivirus expressing control or Nedd4-2 shRNA for 5 days before performing IF. The following day, after NGF starvation in the presence of the caspase inhibitor Z-VAD-FMK, NGF was added for different times to induce TrkA activation and internalization. Cells were then fixed with $4 \%$ paraformaldehyde (PFA) in PBS for 5 min, quenched with $50 \mathrm{mM} \mathrm{NH}_{4} \mathrm{Cl}$ for 10 min, blocked and permeabilized with PBS containing 5\% NGS, $0.1 \%$ Tween-20, $0.1 \%$ TritonX-100 for $30 \mathrm{~min}$, and incubated with primary antibodies overnight at $4^{\circ} \mathrm{C}$. The following day, cells were washed three times with PBS, incubated with the corresponding fluorescent dye-conjugated secondary antibody at room temperature for $40 \mathrm{~min}$, and washed with PBS three times. Images were collected with a Leica confocal microscope. To avoid bleed-through between channels, each channel was acquired separately in the 
co-localization experiments. For co-localization analysis, images of the cells were processed with ImageJ $(\mathrm{NIH})$, using similar threshold settings for all pictures analyzed. Quantification of the processed images was performed with a custom-written program in Matlab.

\subsubsection{Biotinylation Assay}

Sulfo-NHS-SS-biotin reagent (sulfosuccinimidyl-2-[biotinamido]ethyl-1,3-dithiopropionate) is a thiol-cleavable, water soluble and amine-reactive biotinylation reagent that contains an extended spacer arm to reduce the steric hindrances associated with avidin binding (Figure M1).

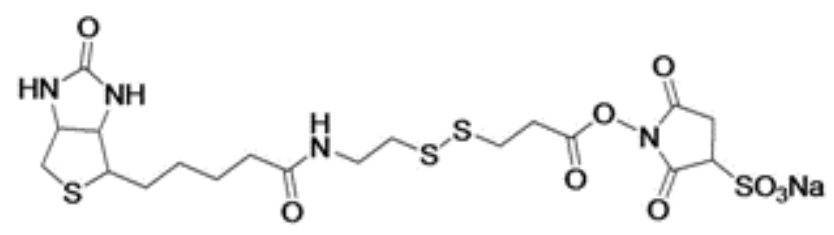

Figure M1. Sulfo-NHS-SS-biotin

Sulfo-NHS-SS-biotin is an excellent reagent for labeling cell surface protein in light of the following characteristics: the N-hydroxysulfosuccinimide (NHS) ester group on Sulfo-NHS-SS-biotin reacts with the $\varepsilon$-amine of lysine residues present in protein to produce a stable product. Sulfo-NHS-SS-biotin is cell membrane-impermeable due to its sodium sulfonate group, and the biotinylated protein can be pulled down and detected using agarose- labeled NeutroAvidin. Moreover, it is possible to cleave the biotin group from the crosslinked target protein under reducing conditions using DTT with Sulfo-NHS-SS-biotin; it is also applied to test the internalization of surface proteins in response to an internalization-induced stimulation. Therefore, the labeling of cell surface proteins with Sulfo-NHS-SS-biotin is a good option for discriminating cell surface proteins from total cell proteins (Figure M2). 


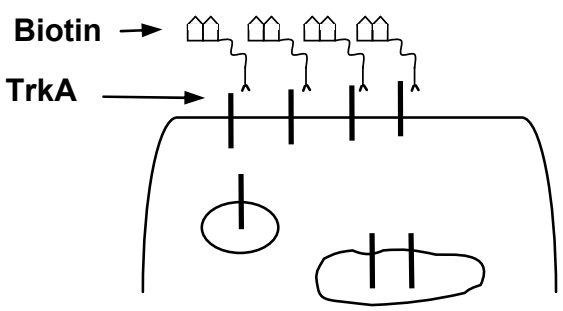

Figure M2. Sulfo-NHS-SS-biotin is an excellent reagent to label cell surface proteins.

\subsubsection{Surface Biotinylation Assay}

DRG neurons from rat embryos were cultured on a 12-well plate at a density 100,000 per well and were infected with control and Nedd4-2 shRNA lentivirus to downregulate the level of Nedd4-2 before NGF-starvation overnight. Cells were stimulated without or with NGF (50 ng/ml) for different times (15 $\mathrm{min}$ and $60 \mathrm{~min}$ ) to induce the internalization of activated TrkA neurotrophin receptors. To have enough amounts of cell-surface labeled proteins, two wells of cells were used for each time-point. Then, cells were washed sequentially with room-temperature and cold PBS, $1 \mathrm{mM} \mathrm{CaCl}_{2}, 0.5 \mathrm{mM} \mathrm{MgCl}_{2}$, chilled on ice, and incubated in $0.5 \mu \mathrm{g} / \mathrm{ml}$ Sulfo-NHS-SS-biotin for $20 \mathrm{~min}$ at $4{ }^{\circ} \mathrm{C}$ to label the membrane proteins (Figure M3). Free biotin was quenched with $0.1 \mathrm{M}$ Glycine for $15 \mathrm{~min}$ at $4{ }^{\circ} \mathrm{C}$. Cells were then washed twice with cold PBS, $1 \mathrm{mM} \mathrm{CaCl}, 0.5 \mathrm{mM} \mathrm{MgCl}_{2}$ and lysed. Biotinylated proteins were isolated from the total cell lysate by immobilization on NeutroAvidin-beads for at least 3 hours at $4^{\circ} \mathrm{C}$. Beads were washed three times with lysis buffer and mixed with $20 \mu \mathrm{l}$ of $2 \times$ SDS sample buffer before boiling for $7 \mathrm{~min}$. Proteins were subjected to SDS-PAGE and immunoblotted with the corresponding antibodies.

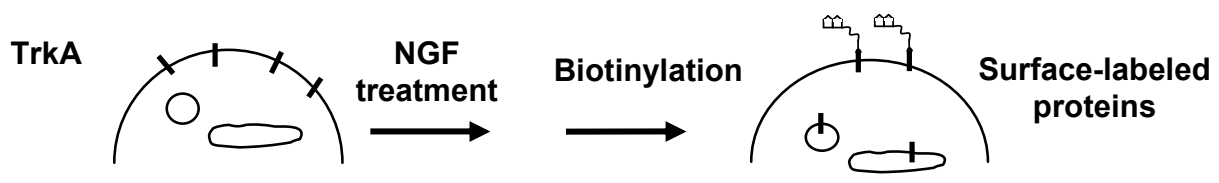

Figure M3. Surface biotinylation assay 


\subsubsection{Internalization Assay}

Unlike the surface biotinylation sssay, which tests surface protein after NGF stimulation, the internalization assay tested internalized surface protein in response to NGF. Briefly, infected DRG neurons were grown in mass cultures, infected with control and Nedd4-2 shRNA lentivirus to downregulate the level of Nedd4-2, and then NGF-starved overnight. As outlined in Figure M4, Neurons were subjected to biotinylation on ice with the reversible membrane-impermeable derivative of Sulfo-NHS-SS-biotin. Cells were then washed twice to eliminate free biotin and incubated in pre-warmed medium at $37^{\circ} \mathrm{C}$ without or with NGF for different times (15 $\mathrm{min}$ and $60 \mathrm{~min}$ ) to induce the internalization of biotinylated surface protein. To remove the remaining surface-labeled proteins, the cell surface-bound biotin was cleaved by reducing its disulfide linkage with $50 \mathrm{mM}$ DTT for 15 min at $4{ }^{\circ} \mathrm{C}$. Cells were lysed and biotinylated proteins were precipitated by NeutroAvidin-beads, eluted from the beads, resolved by SDS-PAGE, and immunoblotted with the corresponding antibodies.

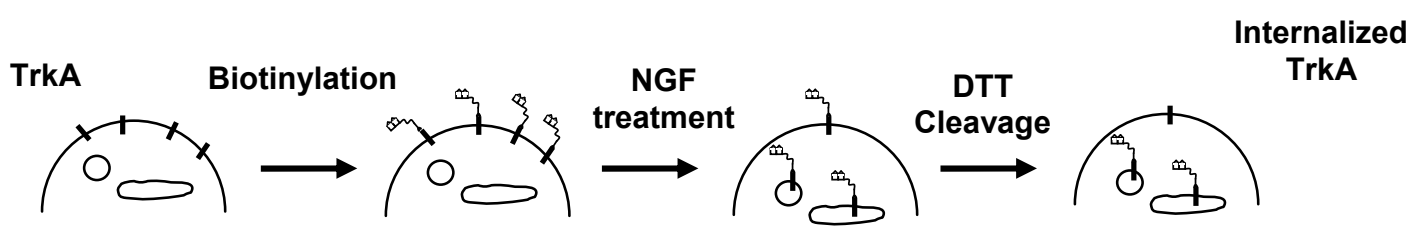

Figure M4. Internalization assay

\subsubsection{Degradation Assay}

Infected DRGs with control and Nedd4-2 shRNA lentivirus were NGF-starved overnight. The following day, neurons were washed with PBS $1 \mathrm{mM} \mathrm{CaCl}_{2}, 0.5 \mathrm{mM} \mathrm{MgCl}_{2}$, chilled on ice, and biotinylated using $0.5 \mu \mathrm{g} / \mathrm{ml}$ Sulfo-NHS-SS-biotin for $20 \mathrm{~min}$ at $4{ }^{\circ} \mathrm{C}$. Cells were sequentially washed with cold and room-temperature PBS, $1 \mathrm{mM} \mathrm{CaCl}_{2}$, and $0.5 \mathrm{mM}$ $\mathrm{MgCl}_{2}$ and were incubated in pre-warmed medium at $37{ }^{\circ} \mathrm{C}$ w/o NGF (50 ng/ml) for different times (15 $\mathrm{min}$ and $60 \mathrm{~min}$ ) to allow the biotinylated receptors to become 
internalized and degraded (Figure M5). Subsequently, cells were washed and lysed using lysis buffer and the biotinylated proteins were precipitated with NeutroAvidin-beads, washed and subjected to SDS-PAGE, and immunoblotted using different antibodies. With this method it was possible to detect surface-labeled proteins that had not been degraded at different time-points, regardless of whether the proteins had been internalized or whether they had returned to the plasma membrane.

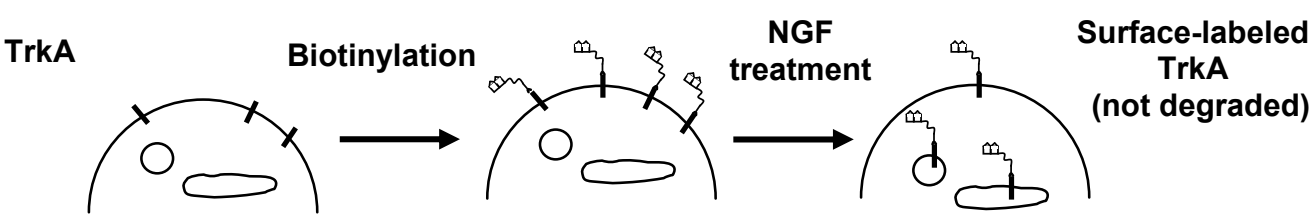

Figure M5. Degradation assay

\subsubsection{TrkA ubiquitination}

\section{Total TrkA ubiquitination in DRGs in response to NGF stimulation}

To test TrkA ubiquitination in rat and mouse DRGs neurons, we first obtained and cultured DRG neurons. Rat DRG neurons were obtained from E15.5 embryos, after which the neurons were infected with lentivirus to downregulate the level of Nedd4-2 and starved overnight on DIV9. The following day, NGF was added for 10 and 30 min to stimulate TrkA. DRG neurons from mouse embryos were obtained and cultured like rat DRG neurons, but without infection. Cells were lysed and TrkA was immunoprecipitated using anti-Trk (C-14) conjugated agarose. TrkA pulled-down complexes were recovered, washed, and resolved using SDS-PAGE and reprobed for anti-Ubiquitin antibody.

\section{TrkA ubiquitination in early endosomes in response to NGF stimulation}

To test the ubiquitination of TrkA in early endosomes in DRGs neurons, we first obtained the early endosomal compartment by immunoprecipitation, using the membrane marker Rab5. Then, the early endosomal compartment was solubilized and TrkA neurotrophin 
receptors were immunoprecipitated for the ubiquitination test (Figure M6). Cultured DRG neurons were prepared as mentioned in the total TrkA ubiquitination assays and then the cells were washed with $1 \mathrm{ml}$ cold PBS followed by centrifuging for $5 \mathrm{~min}$ at $300 \mathrm{xg}$ at $4^{\circ} \mathrm{C}$ to obtain cell pellets. $800 \mu \mathrm{l}$ of homogenization buffer with inhibitors was added to make up a cell suspension solution and cells were homogenized by passing them through a $22 \mathrm{G}$ needle $(1 \mathrm{ml})$ on ice 30 times, followed by 5 times using a $26 \mathrm{G}$ needle to homogenize the cells without solubilizing the membranes. The supernatants containing the membranes were obtained by centrifugation for $10 \mathrm{~min}$ at $800 \mathrm{xg}$ at $4^{\circ} \mathrm{C}$, leaving $40 \mu \mathrm{l}$ apart as input. The remaining supernatants were used to immunoprecipitate early endosomes by incubation with anti-Rab5 antibody overnight, followed by incubation with protein-G Sepharose for another 2 hours. The immunoprecipitated complexes were recovered by centrifugation at $5000 \mathrm{rpm}$ for $1 \mathrm{~min}$ and were resuspended in $750 \mu$ l of lysis buffer containing protease inhibitors in the presence of $0.1 \%$ SDS and incubated for 15 min at $4^{\circ} \mathrm{C}$ to release the proteins specific to the early endosomall compartment into the supernatants, which were used for immunoprecipitating TrkA using anti-Trk (C-14) conjugated agarose. TrkA pulled-down complexes from the early endosomal compartment were recovered, washed, and resoveld using SDS-PAGE and reprobed with anti-Ubiquitin antibody.

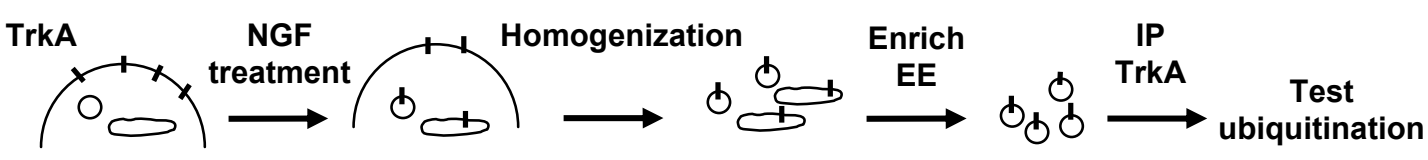

Figure M6. Scheme showing the procedure used to assess TrkA ubiquitination from early and late endosomes.

\subsubsection{NGF-mediated signalling}

DRG neurons from E15.5 rat embryos or E13.5 mouse embryos were cultured in the presence of NGF to select TrkA-expressing neurons. Once the cultures were free of 
dividing cells, the rat DRG neurons were infected with lentivirus expressing control or Nedd4-2 shRNA for 5 days in order to downregulate the level of Nedd4-2. Using GFP as an indicator of the efficiency of infection, by 5 days after infection at least $80 \%$ of the cultured neurons were green under the fluorescence microscope. DRG neurons from mice were cultured like the neurons from rat DRGs but they were not infected. The day before the experiment was performed, cultured DRG neurons were starved of neurotrophins overnight to stop the constitutively activated signals. The starvation was performed in the presence of $20 \mu \mathrm{M}$ Z-VAD-FMK apoptotic inhibitor to prevent the cell death caused by the absence of NGF. On the following day, NGF was added to the medium for $5,15,30,45$ and 60 min to induce TrkA activation, after which stimulated neurons were immediately chilled on ice, washed with cold PBS and lysed in $2 \times$ SDS loading buffer. The lysates were denatured by heating at $100{ }^{\circ} \mathrm{C}$ for $7 \mathrm{~min}$ and then resolved using SDS-PAGE. Western blots were performed to analyze the level of p-Trk, p-Akt (Ser473), p-MAPK, and p-PLC $\gamma$ for the activation of the kinases; Nedd4-2 for the efficiency of infection, and $\beta$-Tubulin III as an indicator of sample loading.

\subsubsection{Retrograde transport}

To mimic the activation of TrkA on the distal axon of DRG neurons in vivo microfluidic chambers (Standard Neuron Device $450 \mu \mathrm{M}$, Xona Microfluidics) were used to separate the environment of cell bodies from the axons (Figure M7). The microfluidic chambers were assembled on glass coverslips previously treated with $1 \mathrm{mg} / \mathrm{mL} P D L$ and placed in a $10 \mathrm{~cm}$ Petri dish the day before dissection of the DRGs, as indicated by the manufacturer. $200 \mu \mathrm{l}$ of NB medium was added to the top wells while $150 \mu \mathrm{l}$ was added to the bottom wells and these were incubated overnight in the incubator to ensure that there would not be any leakage of liquid. The separation of the upper compartment and the lower compartment was monitored by the level of the liquid (Figure M7A). The following day, any chamber with signs of leakage was discarded. Then, the chambers were coated with Matrigel and $10 \mu \mathrm{l}$ of plating medium with NGF containing $2 \times 10^{5}$ rat DRG neurons was 
plated in the hole of the groove on the upper side of each chamber to assess retrograde transport. Cells were allowed to adhere to the coverslip in the groove for $10 \mathrm{~min}$ and additional plating medium was added to each well of the chamber. To isolate each chamber, the volume in the wells on one side of the device was higher than the other side. The plating medium was replaced on the following day with NB medium with NGF and 5FU to kill dividing cells. After three days of culture, the separation of axons of the DRG neurons from the cell bodies was observed (Figure M7B), then, lentivirus infections were performed as a standard procedure at this point. To determine whether the neurons had undergone retrograde transport, red fluorescent beads ( $40 \mathrm{~nm}$, Invitrogen) were added to the distal compartments and the next day the cell body compartment was monitored for red fluorescence. The chambers with a large amount of fluorescence in the proximal compartment were used for the experiments. To test retrograde transport, neurons were NGF-starved overnight as previously described and followed by NGF stimulation in different compartments to test the activation of TrkA.

A

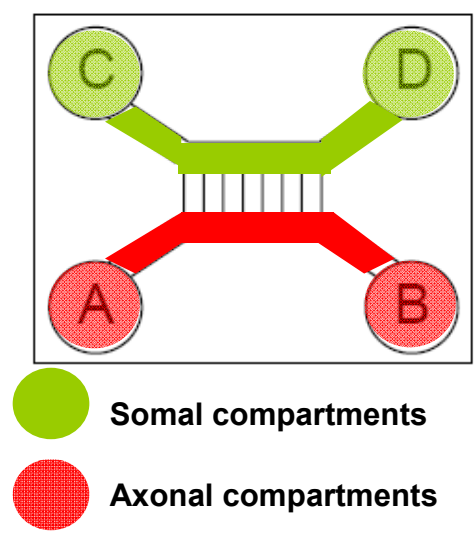

B

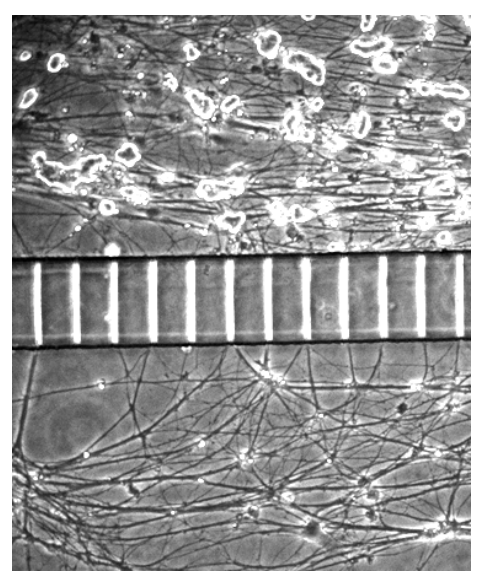

Figure M7. Microfluidic chambers isolate neuronal soma from axons.

(A) Picture of a microfluidic chamber.

(B) DRG neurons were plated in the upper wells (cell body compartment) and axons crossed the microgrooves to localize in the lower wells (axonal compartment). 


\subsubsection{TrkAP782S Knock-in mice generation}

To generate a targeting construct for TrkAP782S knock-in mice, a genomic clone from 129SV/J mice containing exon 17, which encodes the C-terminus of TrkA, was used. The triplet coding for proline (CCA) from this DNA clone was mutated to serine (TCG) using site-directed mutagenesis, which was confirmed by sequencing and the disappearance of a BstXI restriction site. A neomycin-resistance gene flanked by FRT sites containing a loxP site was inserted downstream from exon 17. The truncated diphtheria toxin gene was placed at the end of the short arm for negative selection. The targeting vector was linearized at a unique upstream BstXI site and was electroporated into CJ7 embryonic stem (ES) cells, as previously described (Tessarollo 2001). Ninety-seven G418-resistant colonies were screened for homologous recombination by Southern blot analysis with an external probe. This probe was a $1.2 \mathrm{~Kb}$ DNA fragment that was excised from the pmA5 plasmid by Sacl digestion. A total of 7 clones showed the correct band pattern with the external probe and a Neo probe. Three ES clones were injected into C57BL/6 blastocysts to generate chimeric mice. The chimeras were bred with C57BL/6 mice that expressed the Flp recombinase driven by the actin promoter to remove the Neo cassette and all three gave germline transmission. TrkAP782S genotyping was performed by PCR with tail genomic DNA and the following two primers: TrkA-Pvul-F (5'-ggcgcaggcgatcgagtgtatc-3') and mTrkA-1-B (5'-cctccgcaatggacaggag-3'). A $1.2 \mathrm{~Kb}$ product was amplified from the WT allele, and a $1.4 \mathrm{~Kb}$ product was amplified from the mutant allele. Confirmation of the genotyping was accomplished by digestion of the PCR product with the BstXI restriction enzyme, which digests the wt alleles but not the mutant alleles. In addition, digestion with the Pstl restriction enzyme was performed to confirm that the lack of digestion of mutant TrkA DNA with BstXI was not due to problems with the amplified DNA.

All animals were housed and bred in Specific Pathogen Free (SPF) Animal Facility of the University of Salamanca. All animal care and procedures were done in accordance with protocols approved by the Bioethics Committee of the University of Salamanca and following the European Community guidelines. 


\subsubsection{DRG neuron survival assay}

NGF- and BDNF-dependent rat DRG neurons plated on 24 well-plates were transfected at DIV4-5 with plasmids expressing both GFP and control or Nedd4-2 shRNA using Lipofectamine 2000 (Invitrogen). The following day, the mediUM was replaced with fresh medium containing NGF or BDNF for 48 hours, after which both neurotrophins were withdrawn. Transfected cells were recognized by GFP expression and apoptotic neurons, identified by fragmented or condensed nuclei using Hoechst 33342, were scored 72-120 hours after the corresponding withdrawal of neurotrophin. Images were taken from random fields using a Leica DMI3000B microscope equipped with a Leica DFC300FX camera. To avoid variability among the independent experiments, a figure of $100 \%$ was given to the percentage of apoptosis after counting all cells.

For the E13.5 wild-type and knock-in mice, DRG neurons were dissected and 10.000 cells were plated on a 24-well plate with $0,1,5,10$ or $25 \mathrm{ng} / \mathrm{ml}$ of NGF. The following day, the medium was changed to NB medium containing $5 F U$, keeping the same amount of NGF as that plated. Images were taken from 4 random fields after 6-9 days and living cells were counted. The number of live cells growing in $25 \mathrm{ng} / \mathrm{ml}$ of NGF was considered $100 \%$.

\subsubsection{RNA extraction, Reversed Transcript and qPCR}

DRG neurons from E13.5 wild-type and knock-in TrkP782S mice were dissected as described above and the isolated DRGs were homogenized in Trizol (Gibco) and frozen at $-80^{\circ} \mathrm{C}$ for future RNA extraction. Total RNA was extracted using chloroform and isopropyl alcohol according to the manufacturer. Normally, $0.2-0.5 \mu \mathrm{g} / \mu \mathrm{l}$ of total RNA with high RNA quality (A260/A280>1.8) was obtained. The first cDNA was synthesized using GoScript ${ }^{\mathrm{TM}}$ Reverse Transcription System (Promega) with random primers. The cDNA sample concentration was determined by measuring the absorbance at $260 \mathrm{~nm}$ using a NanoDrop 2000C Spectrophotometer (Thermo Scientific). Quantification of the PCR products (TrkA and $\beta$-actin, as reference gene) was accomplished with a standard curve using 
SYBR-Green. The SYBR-Green was included in a 2 x Master Mix (Applied Biosystems, Madrid, Spain) (SYBR Green dye, dNTPs, Passive Referente (ROX), AmpliTaq Gold DNA polymerase). The final volume of each reaction was $20 \mu \mathrm{l}$ : $10 \mu \mathrm{l}$ of Master Mix, $0.8 \mu \mathrm{l}(16$ pMoles) of each oligonucleotide, $7.4 \mu \mathrm{l}$ of distilled water and $1 \mu \mathrm{l}$ of cDNA at a concentration of $100 \mathrm{ng} / \mu \mathrm{l}$. A standard curve was constructed for each experiment by serial dilutions of cDNA: $0.01 \mathrm{ng} / \mu \mathrm{l}, 0.001 \mathrm{ng} / \mu \mathrm{l}, 0.0001 \mathrm{ng} / \mu \mathrm{l}$ and $0.00001 \mathrm{ng} / \mu \mathrm{l}$. The amplification reaction took place in an $A B I$ Prism 7300 detection system (Applied Biosystems), with the following conditions: $10 \mathrm{~min}$ at $95^{\circ} \mathrm{C}$ followed by 36 cycles of $15 \mathrm{~s}$ at $95^{\circ} \mathrm{C}$ and $1 \mathrm{~min}$ at $53^{\circ} \mathrm{C}$ (TrkA and $\beta$-actin). The primers for TrkA and $\beta$-actin and their amplicons are listed in Table M6. Three PCR reactions were performed for each sample per plate, and each experiment was repeated three times. Data are represented as means \pm SEM and were analyzed using a two-tailed Student's t-test $(n=3)$. Significance for all tests was assumed at $p<0.05$.

Table M6. List of Primers

\begin{tabular}{llll}
\hline Name & Amplicon & Sense & Primer sequence \\
\hline 3-Actin & \multirow{2}{*}{$104 \mathrm{bp}$} & Forward & 5'-AGC CAT GTA CGT AGC CAT CC-3' \\
& & Reverse & 5'-ACC CTC ATA GAT GGG CAC AG-3' \\
\hline TRKA & $156 \mathrm{bp}$ & Forward & 5'-CGA TCC ACC AAG CCA GTA TT-3' \\
& & Reverse & 5'-ATC CTG GGT CTT TCC AGC TT-3' \\
\hline
\end{tabular}

\subsubsection{Thermal Response - Tail Flick Test}

An automatic tail-flick apparatus (Ugo Basile Tail Flick) was used to assess the thermal nociceptive threshold in the mice. Before performing the test with WT and KI mice, the infrared heat stimulus was adjusted in different sets of WT animals to an intensity sufficient elicit a tail-flick latency around 5-6 seconds. The time from the start of the heating till the point when the tail was removed from the heating source was read by the apparatus automatically. 3- to 4-month-old WT and KI male mice (25-30 g) were 
habituated for 30 min in individual cages and manipulated for 3-4 days until they were not stressed by the manipulation. Mice were gently held with a towel and the source of heating was applied to a point marked at $4 \mathrm{~cm}$ from the tip of the tail. To avoid tissue damage, a maximum cutoff of 10 seconds was used. Data were collected, presented as means \pm SEM and tested for statistical significance using Student's t-test.

\subsubsection{Thermal Response - Cold plate Test}

Mice were placed within a transparent Plexiglas chamber $(16 \times 16 \times 27 \mathrm{~cm}, \mathrm{l}-\mathrm{w}-\mathrm{h}$ dimensions) on ice and the temperature of the bottom of the chamber was maintained at $4^{\circ} \mathrm{C}$. The latency to respond with one of the following types of behavior was measured with a stopwatch: licking, lifting, guarding, shaking or biting of the hind paw, or jumping. This test did not result in any signs of tissue injury, such as swelling or post test grooming. Data were collected, presented as means \pm SEM and tested for statistical significance using a Student's t-test.

\subsubsection{Mechanical Response - Paw Pressure Test}

Paw pressure experiments were employed to test how the mice responded to mechanical stimulus using an analgesy-meter device. A gradually increasing mechanical force was applied to the hind paw of the mouse until the mouse felt pain: struggling or moving its paw. The force applied until the mice moved their paws was recorded. Both WT and KI male mice were subjected to this test and data were presented as means \pm SEM and analyzed statistically using a Student's t-test. 


\subsubsection{Inflammation Response - Formalin Assay}

To test the response of the mice to chemically induced inflammatory pain, 3-4-month-old WT and KI male mice (25-30 g) were used for the formalin assay. $20 \mu \mathrm{l}$ of $5 \%$ formalin diluted in PBS was injectedin to the right plantar hind paw of each mouse using a 26-G needle. If the injection caused bleeding, the animal was discarded from the data analysis. Immediately after the injection, the mouse was put in a cage and monitored using a video camera for 55 min without disturbance. For analysis, 55 min recording was split into 11 intervals of 5 min each. During every interval, the time the mice spent in licking or biting the injected paw was recorded and summed. All these data were collected, presented as means \pm SEM and statistically analyzed using Student's t-test. Also, based on the response of the mice to the formalin injection their behavior was divided into phase I (0-15 min) and phase II (15-55 min). Phase I corresponded to the burning effect of formalin and phase II to the inflammatory phase. In each phase, the time a mouse spent in licking or biting its paw was recorded and summed. All these data were collected, presented as means \pm SEM and statistically analyzed using Mann-Whitney test.

\subsubsection{In Situ Hybridization}

PO mice from both WT and KI mice were dissected to obtain the spinal cords with the DRGs, fixed in 4\% PFA for 48 hours, cryoprotected overnight in PBS containing $30 \%$ sucrose, embedded in $\mathrm{OCT}$, and frozen at $-20^{\circ} \mathrm{C}$. The tissue was cut in $12-\mu \mathrm{m}$ thick sections, mounted on positively-charged slides and kept at $-20^{\circ} \mathrm{C}$ until use. Sense and antisense probes used for staining mRNA of CGRP and SP were labeled with digoxigenin. To generate the probes, corresponding regions of each mouse gene were PCR-amplified from mouse tissue and cloned in pcDNA3 in the sense and antisense orientations. For hybridization, slides were warmed to room temperature and fixed in $4 \%$ PFA-PBS solution for $10 \mathrm{~min}$ at room temperature, followed by washing with PBS. Subsequently, slides were pretreated with proteinase $\mathrm{K}$ for digestion and fixed again in $4 \%$ PFA-PBS 
solution for another $10 \mathrm{~min}$, followed by washing with PBS. Acetylation was carried out for 10 min at room temperature and the slides were washed with PBS. The probes diluted in hybridization buffer $(200 \mu \mathrm{l} / \mathrm{slide})$ at $0.5-2 \mu \mathrm{g} / \mathrm{ml}$ were denatured at $80^{\circ} \mathrm{C}$ for $5 \mathrm{~min}$, added to the surface of the slides, covered with parafilm, and incubated in a humidified environment overnight at $55^{\circ} \mathrm{C}$. The next day, slides were rinsed with $5 \times$ SSC 5 min at $65^{\circ} \mathrm{C}$, with $2 \times \mathrm{SSC}$ containing $50 \%$ formamide for $30 \mathrm{~min}$ at $65^{\circ} \mathrm{C}$ and with RNAse buffer for $15 \mathrm{~min}$ at $37^{\circ} \mathrm{C}$. The slides were then treated with $20 \mu \mathrm{g} / \mathrm{ml}$ RNAse A for $30 \mathrm{~min}$ at $37^{\circ} \mathrm{C}$ and washed with RNAse buffer for $15 \mathrm{~min}$ at $65^{\circ} \mathrm{C}$, with $2 \times \mathrm{SSC}$ for $15 \mathrm{~min}$ at $37^{\circ} \mathrm{C}$, $0.1 \times$ SSC for $15 \mathrm{~min}$ at $37^{\circ} \mathrm{C}$ and with PBST (PBS containing $0.1 \%$ Tween-20) for $15 \mathrm{~min}$ at room temperature. Sections were blocked for 1.5 hours with PBST containing $10 \%$ NGS (normal goat serum). Then, anti-digoxigenin alkaline phosphatase-conjugated antibodies (anti-DIG-AP Ab) diluted in PBST containing 1\% NGS at 1:3000 were added and incubated overnight at $4^{\circ} \mathrm{C}$. On the next morning the slides were washed with shaking twice with PBST for 1.5 hours each and twice with AP stain buffer for 10 min each time. BM-purple AP substrate (Roche) mixed with $1 \mathrm{mM}$ tetramysole was added to the slides at room temperature in a dark, humidified environment for 24 to 48 hours, depending on the staining signal. The reaction was stopped by rinsing with water several times and with 2 mM EDTA before mounting in Mowiol. The slides were dried overnight and photos were taken using a Leica microscope.

\subsubsection{Neuron counts}

Tissues from P0 WT and KI mice were fixed in PBS containing 4\% PFA, cryoprotected overnight in PBS containing 30\% sucrose, and frozen. Cyrostat sections $(12 \mu \mathrm{m})$ of DRG were prepared and stained with a solution containing $0.5 \%$ cresyl violet, and cells with visible nucleoli were counted as neurons. 
4 Results 


\subsection{Part one: Results from in vitro-cultured rat DRG neurons}

\subsubsection{Nedd4-2 regulates total- and surface-TrkA levels}

It has been previously described that the exogenous expression of Nedd4-2 regulates TrkA, but not TrkB or TrkC, levels (Arevalo et al. 2006b). Since Nedd4-2 is an E3 ubiquitin ligase for TrkA, we first tested whether endogenous Nedd4-2 modulates the levels of total- and surface-TrkA neurotrophin receptors. We addressed this question using in vitro-isolated DRG neurons from E15.5 rat embryos as a model since most DRG neurons express TrkA during development (Snider 1994). We obtained DRG neurons and cultured them in the presence of NGF $(50 \mathrm{ng} / \mathrm{ml})$ to select TrkA-expressing neurons. Then, these neurons were infected with control and Nedd4-2 shRNA lentivirus at the day in vitro (DIV) 4 to modulate Nedd4-2 protein levels. The rate of infection to perform all the biochemical experiments reported henceforth was at least $90 \%$, as seen from GFP expression (Figure R1A).

A

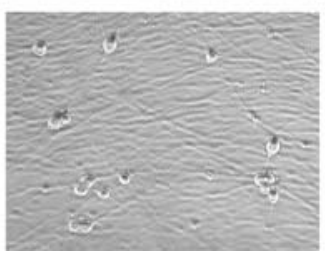

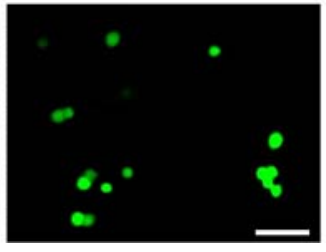

B

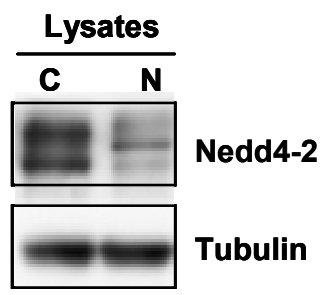

Figure R1. Infection of DRG neurons using Nedd4-2 shRNA lentivirus

(A) Infected DRG neurons were identified by the expression of GFP. Scale bar, $100 \mu \mathrm{m}$.

(B) Cultured DRG neurons were infected on DIV4 with control or Nedd4-2 shRNA lentivirus and extracts were obtained on DIV10 to check the down-regulation of Nedd4-2 using western blot. Tubulin was used as a loading control. Representative western blots are shown. C: Control shRNA lentivirus; N: Nedd4-2 shRNA lentivirus. 
After confirming that the level of Nedd4-2 was dramatically decreased upon infection with lentivirus expressing Nedd4-2 shRNA (Figure R1B), we compared TrkA levels in neurons infected with control and Nedd4-2 shRNA lentivirus and found that Nedd4-2 depletion led to an increase in total TrkA levels (Figure R2A). However, the levels of p75 neurotrophin receptor were not altered by the depletion of Nedd4-2, suggesting that Nedd4-2 did specifically function on TrkA regulation (Figure R2A).

A

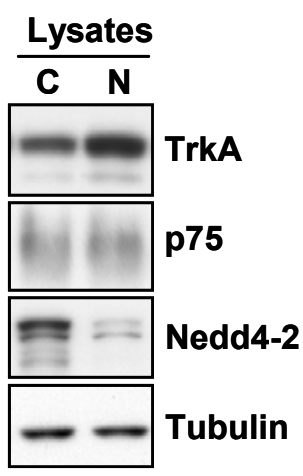

B

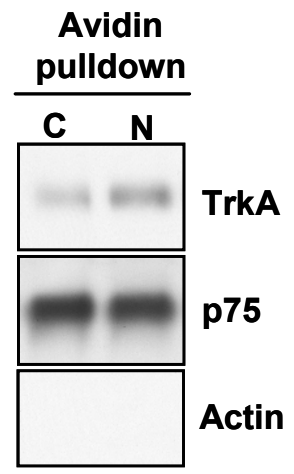

C

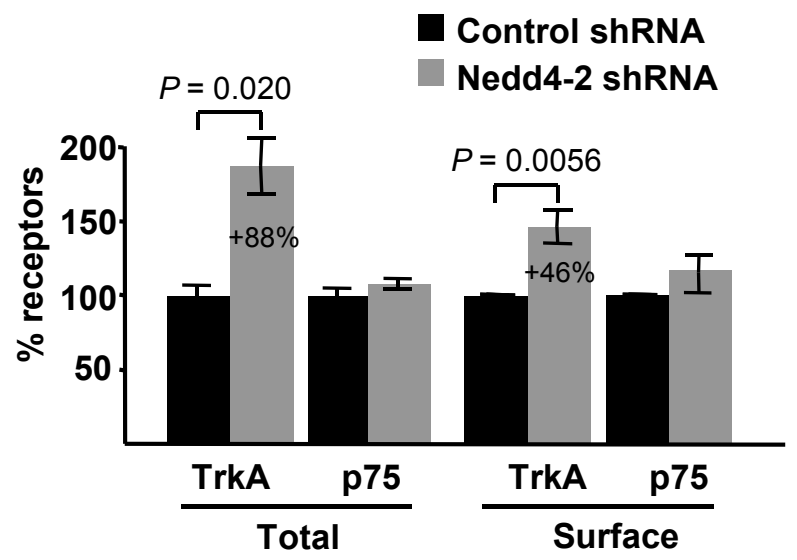

Figure R2. Nedd4-2 depletion increases TrkA levels.

(A) Increased levels of TrkA upon Nedd4-2 depletion. Cultured DRG neurons were infected on DIV4 with control or Nedd4-2 shRNA lentivirus and extracts were obtained on DIV10 to analyze the expression of different proteins using western blot analysis. Tubulin was used as a loading control. Representative western blots are shown. C: Control shRNA lentivirus; N: Nedd4-2 shRNA lentivirus.

(B) Enhanced surface TrkA in Nedd4-2-depleted neurons. Cell surface proteins from NGF-starved DRG neurons infected with control and Nedd4-2 shRNA lentivirus were labeled with biotin and the same amount of proteins was pulled down with Neutroavidin. Western blot analyses were performed with TrkA and p75 antibodies. Representative western blots are shown. C: Control shRNA lentivirus; N: Nedd4-2 shRNA lentivirus.

(C) Quantification of TrkA and p75 proteins in response to Nedd4-2 depletion. Western blots were scanned and quantified using ImageJ software. Data are presented as means \pm SEM. $P$ values were calculated using a two-tailed Student's t-test ( $n=6-8)$. 
To assess whether Nedd4-2 depletion may also influence the surface expression of TrkA, we performed biotinylation experiments. Surface proteins from infected DRG neurons were labeled with sulfo-NHS-SS-biotin, a cell membrane-impermeable compound that only labels cell surface proteins, and pulled-down with Neutroavidin beads followed by western blot analysis. We found that surface-TrkA levels, but not surface-p75 levels, were increased following Nedd4-2 depletion (Figure R2B). Actin was used as a control to confirm that the biotin-labeled proteins indeed came from the cell surface (Figure R2B). Quantification of the data corresponding to the different experiments to detect the total and surface TrkA levels indicated an $88 \%$ increase in total TrkA levels and a $46 \%$ increase in surface TrkA levels in Nedd4-2-depleted neurons as compared to control neurons (Figure R2C). No significant differences in p75 levels were observed (Figure R2C). These results suggest that endogenous Nedd4-2 in sensory neurons regulates the amount of total and surface-bound TrkA, but not p75.

\subsubsection{Nedd4-2 modulates TrkA levels through its degradation}

To address whether Nedd4-2 modulates the degradation rate of TrkA in response to NGF, we performed degradation assays using biotinylation. NGF-dependent DRG neurons were infected with control and Nedd4-2 shRNA lentivirus, starved of NGF overnight in the presence of the anti-apoptotic inhibitor Z-VAD-FMK and protein synthesis inhibitor cycloheximide, and then the cell surface proteins were biotinylated and stimulated with NGF to induce the internalization and degradation of the biotinylated TrkA, as outlined in Figure R3A. After 1 hour of NGF treatment, there was a clear decrease in the amount of biotin-labeled TrkA in the control neurons as a result of TrkA degradation (Figure R3B). However, the amount of biotinylated TrkA neurotrophin receptors from Nedd4-2-depleted neurons was not altered (Figure R3B), suggesting a deficiency of TrkA degradation. Quantification of multiple experiments indicated that no significant degradation of surface TrkA occurred in Nedd4-2-depleted neurons upon NGF stimulation for 1 hour, whereas a $30 \%$ reduction in surface TrkA was noted in control cells (Figure R3C). No differences were detected in the degradation rate of p75 in response to NGF from the control and 
Nedd4-2-depleted neurons (Figure R3D). Therefore, Nedd4-2 levels modulate the degradation rate of TrkA in response to NGF.

A

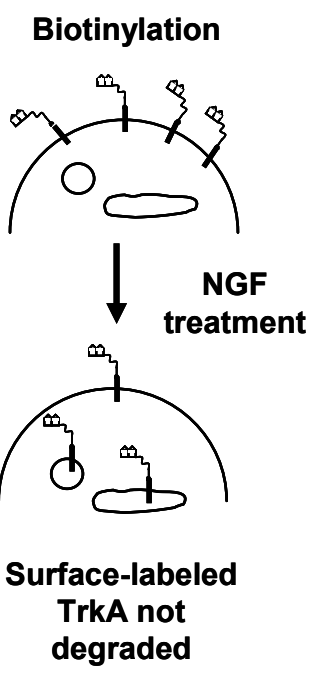

C

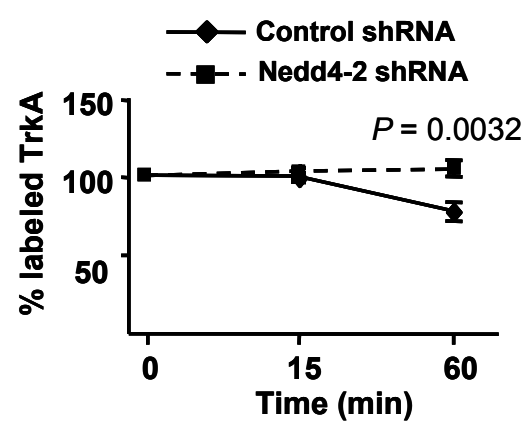

B

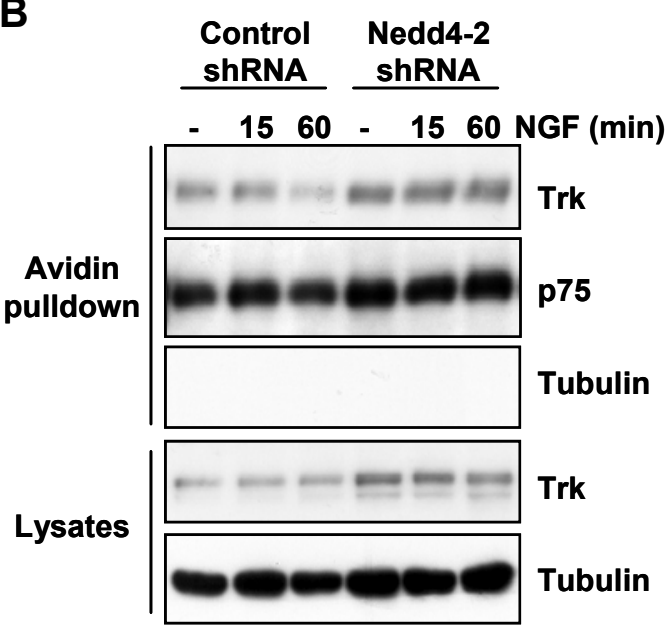

D

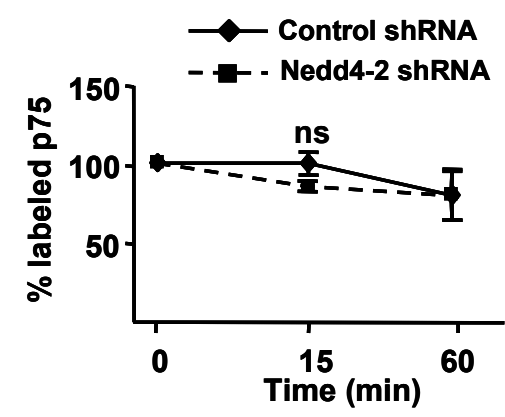

Figure R3. Nedd4-2 regulates TrkA degradation.

(A) A receptor degradation assay was performed as described in Material and Methods and is depicted in the schematic diagram.

(B) Representative western blots are shown. Infected DRG neurons were biotinylated to label surface proteins and treated or not with NGF $(50 \mathrm{ng} / \mathrm{ml})$ for 15 and 60 minutes. An aliquot of the cell lysates was used as loading control and the biotinylated proteins were precipitated with Neutroavidin-agarose. Surface proteins were subsequently subjected to western blot analysis with Trk, p75 and tubulin antibodies. Tubulin was used as a negative control for biotinylated proteins and as a loading control for lysates.

(C) Quantification of surface-labeled TrkA. Data are normalized to the amount of biotinylated TrkA in control and in Nedd4-2-depleted neurons without NGF treatment. Data are presented as means \pm SEM. $P$ values were calculated using a two-tailed Student's t-test $(n=4)$.

(D) Quantification of surface-labeled p75. Data are normalized to the amount of biotinylated p75 in control and in Nedd4-2-depleted neurons without NGF treatment. Data are presented as means \pm SEM. $(n=4)$; ns, not significant. 
The degradation of TrkA protein in response to NGF requires the internalization and trafficking of the receptor to the lysosomes. Any change in this process may affect the outcome of the degradation of TrkA. To assess whether Nedd4-2 protein modulates the internalization rate of TrkA in response to NGF, we performed internalization assays in infected DRG neurons using biotinylation. Infected neurons were NGF-starved as described above and cell surface biotinylation was performed using cleavable biotin, as outlined in Figure R4A. NGF was then applied for 15 or 60 minutes and reducing conditions were applied to strip the biotin from surface proteins that had not been internalized. This treatment allowed the detection of internalized biotinylated proteins, which remained protected from cleavage, upon pulling them down using Neutroavidin beads. We did not observe any significant differences in the rate of internalization of TrkA or p75 in the control and Nedd4-2-depleted cells (Figure R4B, C, D). Thus, these data suggest that Nedd4-2 protein does not regulate the internalization rate of TrkA in response to NGF. These results are consistent with previous observations regarding the lack of differences in the internalization rate of wild-type TrkA and TrkAP782S, a mutant receptor that does not bind Nedd4-2 (Arevalo et al. 2006b). 
A

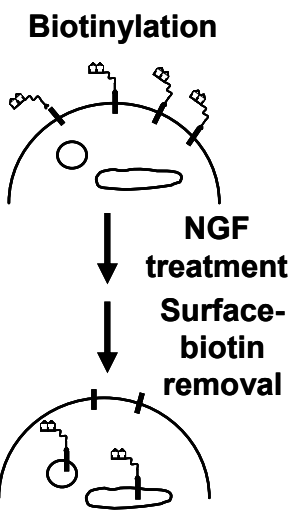

Surface-

labeled

internalized

proteins

C

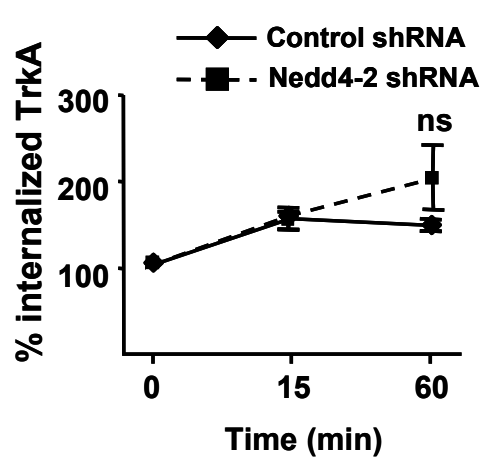

B

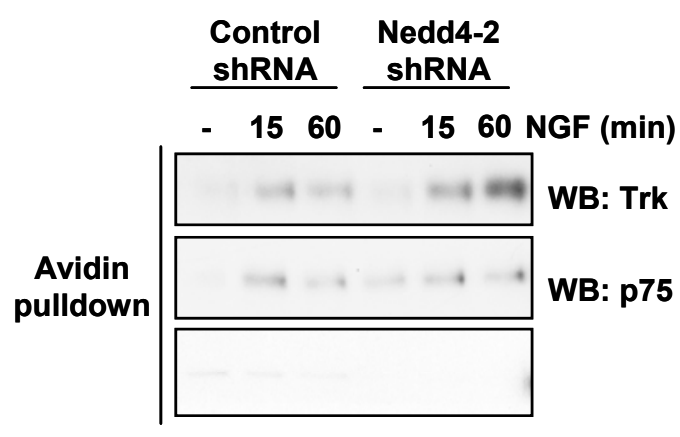

D

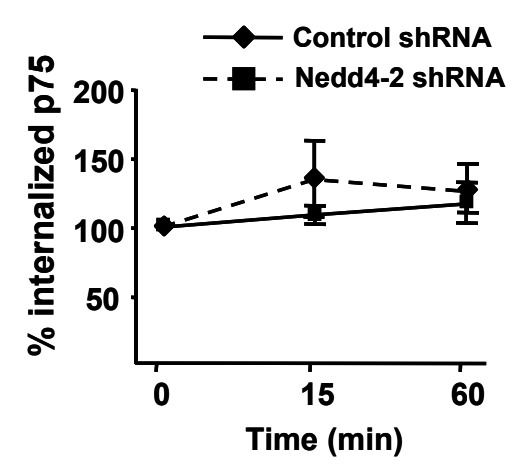

Figure R4. Nedd4-2 does not alter TrkA internalization.

(A) A schematic diagram of the internalization assay using a biotinylation procedure is shown.

(B) Representative western blots are shown. DRG neurons were infected with control and Nedd4-2 shRNA lentivirus. Cells were biotinylated as described in Experimental Procedures and then treated with NGF (50 ng/ml) for 15 and 60 minutes. Biotin from non-internalized proteins was removed using reducing conditions as described in Material and Methods. Cell lysates were prepared, surface proteins were subjected to precipitation with Neutroavidin, and western blot analyses were performed with the corresponding antibodies. Tubulin was used as a negative control for biotinylated proteins.

(C) Quantification of internalized TrkA. Data are presented as means \pm SEM. $(n=3)$; ns, no significant.

(D) Quantification of internalized p75 receptor. Data are presented as means \pm SEM. $(n=3)$. 


\subsubsection{TrkA trafficking at early and late endosomes is regulated by Nedd4-2}

It is known that after NGF stimulation TrkA is internalized and subjected to intracellular trafficking. After observing that Nedd4-2 regulates TrkA degradation, we next addressed whether Nedd4-2 regulates TrkA trafficking using immunofluorescence analysis by detecting the localization of activated TrkA upon depletion of Nedd4-2 in sensory neurons. We performed immunofluorescence analyses to locate activated TrkA upon depletion of Nedd4-2 in sensory neurons by co-staining phosphorylated TrkA with different endosomal markers. Infected NGF-dependent neurons cultured on coverslips and starved as described above, were stimulated with NGF for different times and stained. GFP expression allowed us to detected infected neurons unambiguously. We observed that upon NGF treatment, internalized TrkA co-localized with Rab5 in both the control and Nedd4-2-depleted cells. Upon NGF treatment, more pTrkA was localized in the early endosomes (Rab5 marker) in the Nedd4-2 depleted neurons (Figure R5A). However, considering that surface TrkA levels were increased in the neurons with Nedd4-2 depletion, we normalized pTrkA levels with TrkA surface levels at steady-state in control and Nedd4-2-depleted neurons, as described in Material and Methods. No significant differences in the co-localization of pTrkA with Rab5 were observed between the control and Nedd4-2-depleted neurons at 5 or $30 \mathrm{~min}$ after normalization (Figure R5B). Therefore, these results indicate that depletion of Nedd4-2 does not alter the arrival of activated TrkA at early endosomes, in support of previous data indicating that Nedd4-2 does not modify the internalization of TrkA in response to NGF. 


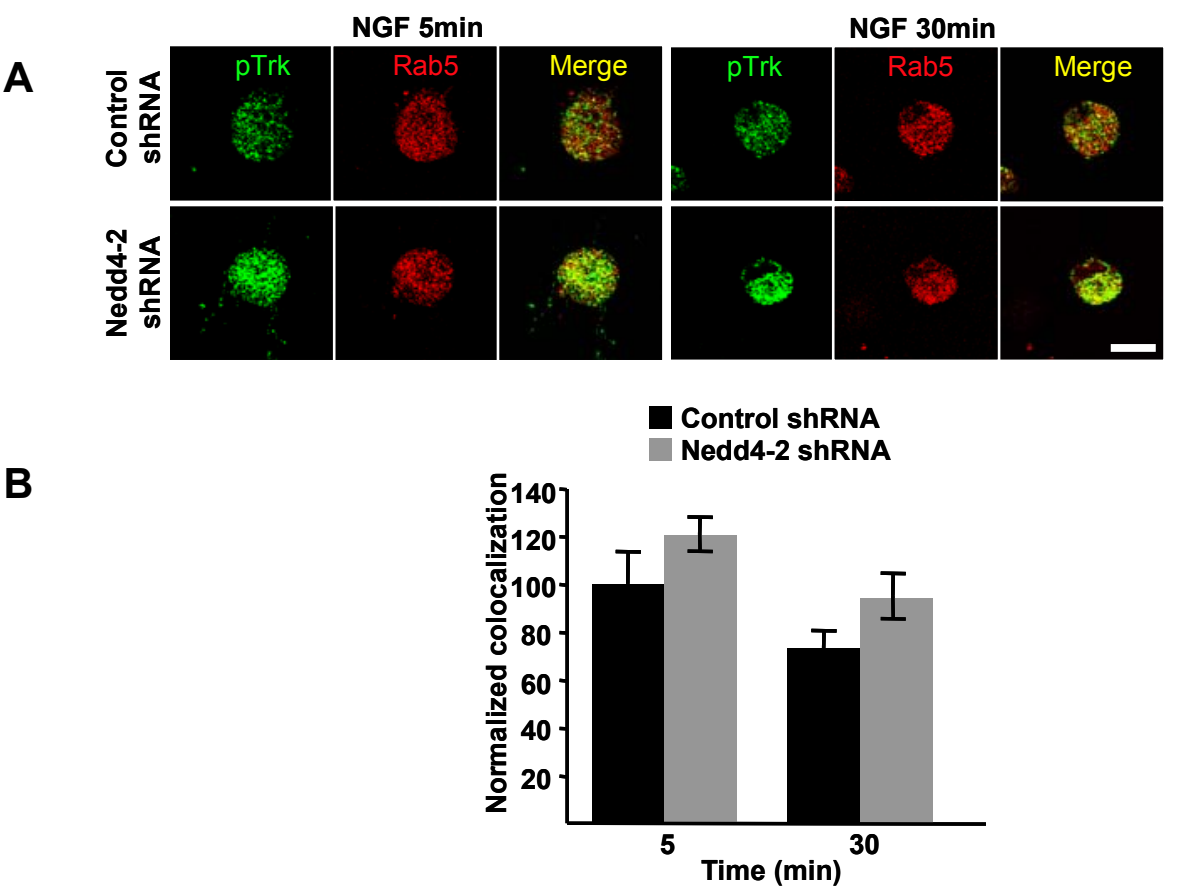

Figure R5. Upon NGF treatment pTrkA localization in early endosomes is not affected by Nedd4-2.

(A) Co-localization of pTrkA with Rab5 compartments upon NGF treatment in control and in Nedd4-2 shRNA-infected DRG neurons. Infected DRG neurons were identified by GFP expression. Immunofluorescence was performed as described in Material and Methods. Images were taken with a confocal microscope. Scale bar, $20 \mu \mathrm{m}$.

(B) Quantification of pTrkA co-localization in Rab5 endosomes. Images were processed using ImageJ and the percentage of co-localization was normalized to the surface levels of TrkA in control and in Nedd4-2-depleted neurons in the absence of NGF. Data are presented as means \pm SEM. $(n=6-9$ neurons/time point).

We next tested whether the level of Nedd4-2 affects the trafficking of activated TrkA to the late endosomes (Rab7 marker). Based on previous data suggesting that Nedd4-2 depletion leads to more TrkA on the surface in the sensory neurons, but does not alter the internalization of TrkA upon NGF stimulation, we expected an increase in activated TrkA levels in late endosomes in a similar way to what was observed for early endosomes Surprisingly, in the Nedd4-2-depleted neurons, less activated TrkA was found in late endosomes after NGF treatment for 10 minutes (Figure R6A). The data were then normalized as stated above and indicated that after 10 min of NGF treatment there was a $40 \%$ decrease in the amount of pTrkA present in the late endosomes of Nedd4-2-depleted cells (Figure R6B). This means that unlike internalization, increased activated TrkA in the 
early endosomes was not efficiently transported to late endosomes. In addition, we analyzed what happened at later time-points upon NGF treatment and found that unlike NGF treatment for 10 minutes, NGF treatment for 30 minutes caused a massive accumulation of pTrkA (three fold increase) in the late endosomes from Nedd4-2-depleted neurons as compared with the control neurons (Figure R6A, B). All these data suggested that Nedd4-2 depletion hindered both the entry of activated TrkA into the late endosomes and the exit from late endosomes to lysosomes.

A
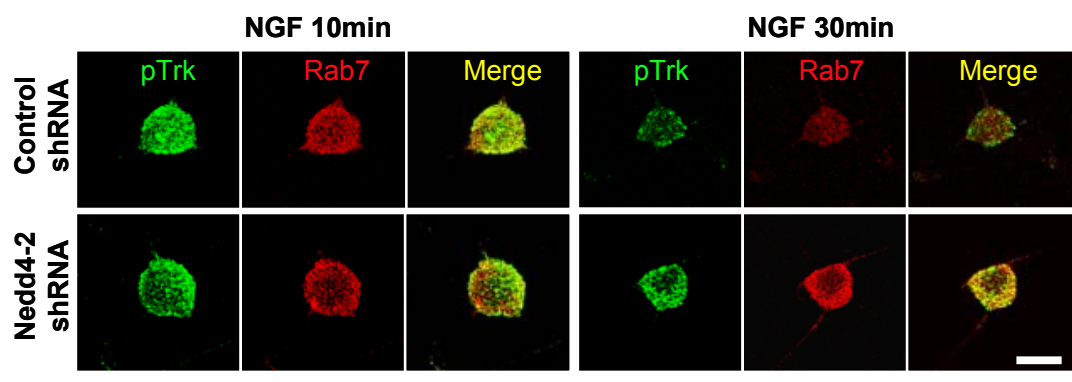

B

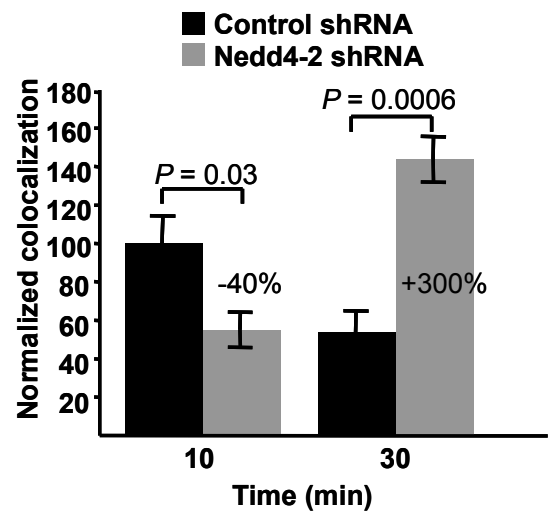

Figure R6. Upon NGF treatment pTrkA localization in late endosomes is regulated

\section{by Nedd4-2.}

(A) Co-localization of pTrkA with Rab7 compartments upon NGF treatment in control and in Nedd4-2 shRNA-infected DRG neurons. Scale bar, $20 \mu \mathrm{m}$.

(B) Quantification of pTrkA co-localization in Rab7 endosomes was performed. Data are presented as means \pm SEM. $P$ values were calculated using a two-tailed Student's t-test ( $n=5-7$ neurons/time point).

To further confirm that the sorting of activated TrkA between early and late endosomes and between late endosomes and lysosomes can be regulated by Nedd4-2, a biochemical approach was applied. Neurons infected with control and Nedd4-2 shRNA lentivirus were NGF-starved overnight, followed by stimulation with NGF $(50 \mathrm{ng} / \mathrm{ml})$ for 10 or $30 \mathrm{~min}$, and the cells were homogenized without detergent to obtain crude membranes. 
Rab5 and Rab7 antibodies were used to pull down the same amount of endomembranes containing these two proteins from control and Nedd4-2-depleted neurons treated with NGF for 10 min (Rab5 immunoprecipitation) or for 30 min (Rab7 immunoprecipitation) and the amount of TrkA protein in the immunoprecipitated membranes was assessed. An increase in TrkA in Rab5- and Rab7-endomembranes from Nedd4-2 depleted neurons as compared to the control neurons was observed after NGF treatment for 10 and $30 \mathrm{~min}$, respectively (Figure R7). Taken together, the results of the co-localization and the biochemical experiments suggest that Nedd4-2 regulates the trafficking of activated TrkA between early endosomes and late endosomes.

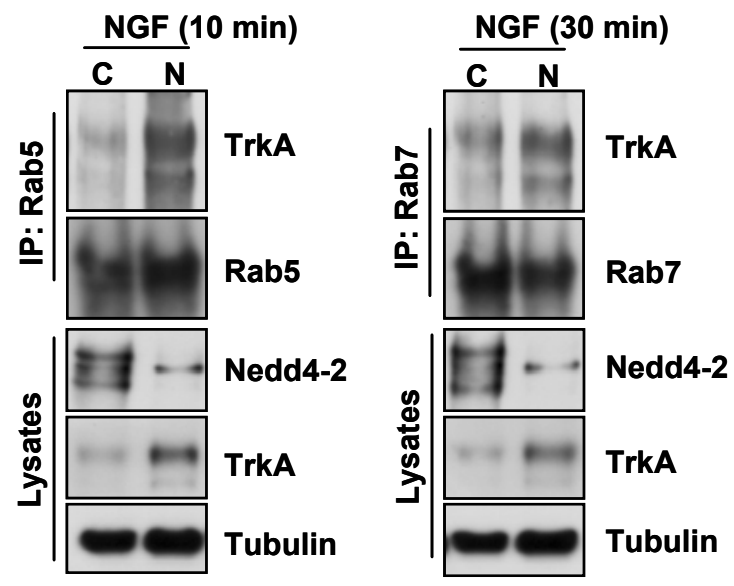

Figure R7. TrkA localization in early endosomes and late endosomes upon NGF stimulation

Infected DRG neurons were treated with NGF for 10 or 30 min and lysates were immunoprecipitated with Rab5 or Rab7 antibodies, respectively. Representative blots are shown $(n=3)$. C: Control shRNA lentivirus; N: Nedd4-2 shRNA lentivirus.

\subsubsection{Increased TrkA recycling to the plasma membrane by Nedd4-2 depletion}

After internalization, activated TrkA that reaches early endosomes can also recycle to the plasma membrane via recycling endosomes (Chen et al. 2005). To address whether Nedd4-2 modulates the recycling of TrkA to the plasma membrane, we performed immunofluorescence analysis in DRG neurons treated with NGF to detect pTrkA in recycling endosomes (Rab11 marker). The images suggested that there may be more 
co-localization of pTrkA within recycling endosomes in Nedd4-2-depleted neurons than in control neurons (Figure R8A). Indeed, quantification analyses of different cells indicated that in Nedd4-2-depleted neurons there was an increased co-localization of pTrkA with Rab11 as compared to control neurons (208\% at $10 \mathrm{~min}$ and $258 \%$ at $30 \mathrm{~min}$ ) (Figure R8B). These data suggest that more TrkA protein recycled to the cell surface when Nedd4-2 is reduced.

A

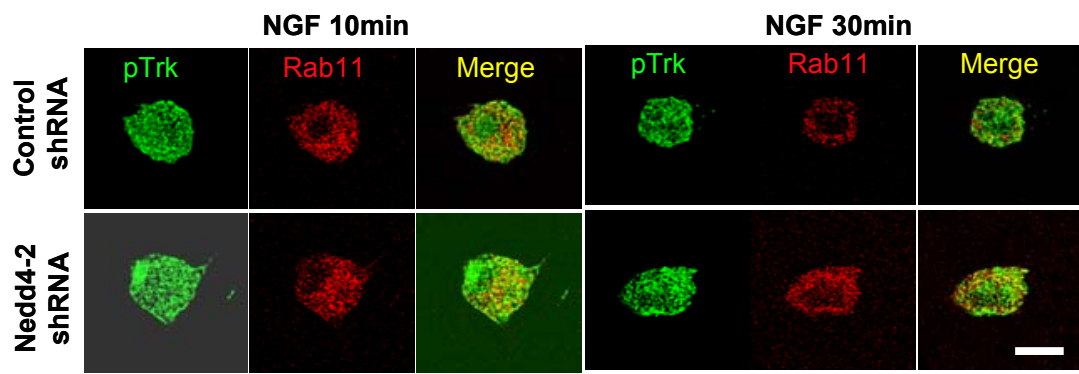

- Control shRNA

B

Nedd4-2 shRNA

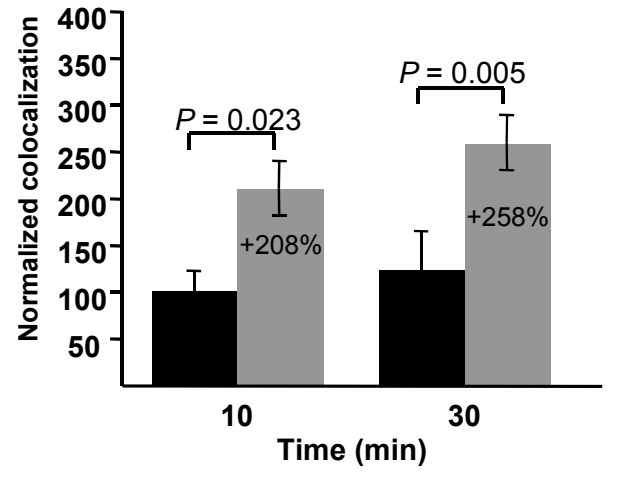

Figure R8. Enhanced pTrkA recycling in Nedd4-2-depleted DRG neurons

(A) Co-localization of pTrkA with Rab11 compartments upon NGF treatment in control and in Nedd4-2 shRNA-infected DRG neurons was performed. Scale bar, $20 \mu \mathrm{m}$.

(B) Quantification of pTrkA co-localization in Rab11 recycling endosomes. Data are presented as means \pm SEM. $P$ values were calculated using a two-tailed Student's t-test ( $n=5-7$ neurons/time point).

Further confirmation for this hypothesis came from biotinylation experiments detecting the amount of TrkA at the cell surface at different time points upon NGF treatment, as outlined in Figure R9A. Upon NGF treatment, we observed a reduction over time in the amount of surface TrkA in control cells (Figure R9B, C). However, in Nedd4-2-depleted neurons this reduction in surface-TrkA levels in response to NGF stimulation was attenuated (Figure $\mathrm{R} 9 \mathrm{~B}, \mathrm{C})$, suggesting that TrkA recycling may be enhanced. In contrast, the reduction in 
p75 from the cell surface in response to NGF was not affected by Nedd4-2 depletion

(Figure R9B, D). Therefore, it may be surmised that TrkA recycles more and is increased in the plasma membrane when Nedd4-2 is depleted, further supporting a potential role for Nedd4-2 in TrkA intracellular trafficking.

A
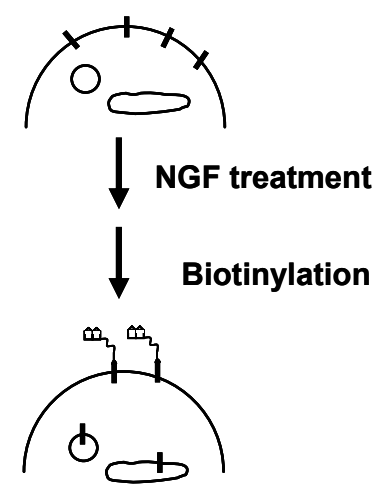

\section{Surface-labeled proteins}

C

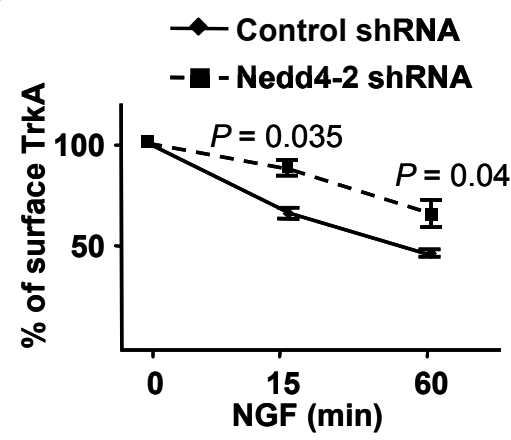

B

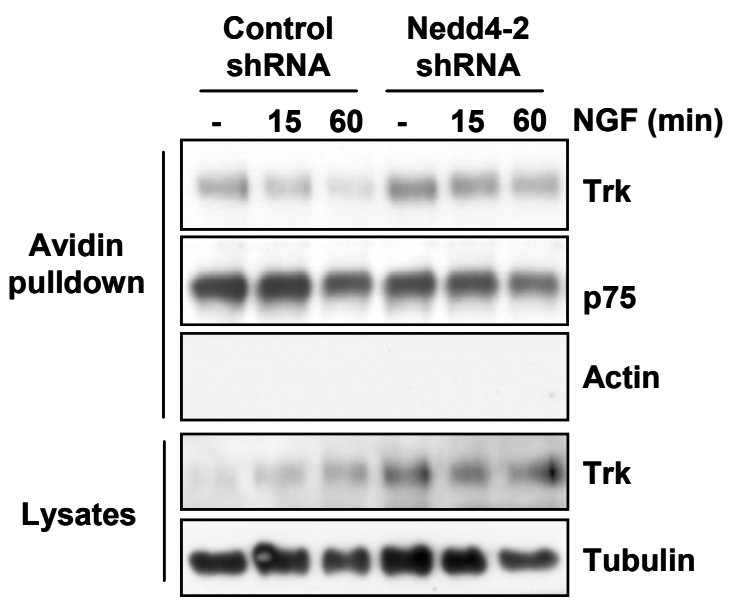

D

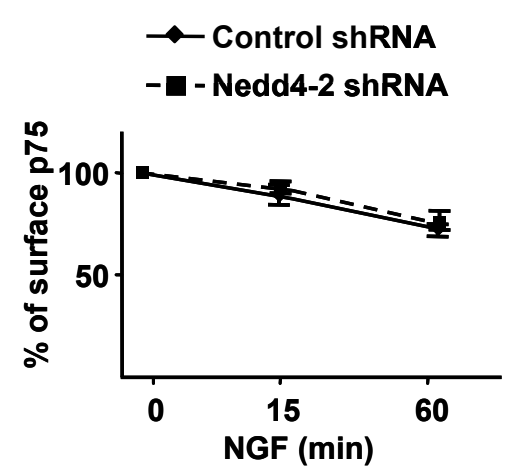

Figure R9. Enhanced surface TrkA in Nedd4-2-depleted DRG neurons upon NGF treatment

(A) A schematic diagram of the surface expression assay using the biotinylation procedure in response to NGF is shown.

(B) Representative western blots are shown. DRG neurons were infected with control and Nedd4-2 shRNA lentivirus. Cells were treated with NGF $(50 \mathrm{ng} / \mathrm{ml})$ for 15 and 60 minutes and then biotinylated as described in Material and Methods. Cell lysates were prepared; surface proteins were subjected to precipitation with neutroavidin-agarose, and western blot analyses were performed with the corresponding antibodies. Tubulin and actin were used as a loading control for lysates and as a negative control for biotinylated proteins, respectively.

(C) Quantification of surface TrkA upon NGF treatment. Data are presented as means \pm SEM. $P$ values were calculated using atwo-tailed Student's t-test $(n=3)$.

(D) Quantification of surface p75 upon NGF treatment. Data are presented as means \pm SEM. $(n=3)$. 


\subsubsection{Nedd4-2 modulates TrkA ubiquitination in early endosomes and late endosomes}

To address whether Nedd4-2-mediated ubiquitination of TrkA may be responsible for the impaired trafficking of activated TrkA between different endosomal compartments, we performed ubiquitination assays. Using cultured DRG neurons infected with control and Nedd4-2 shRNA lentivirus we treated the cells with NGF for 10 min and performed immunoprecipitation of total TrkA. Surprisingly, we did not observe a decreased TrkA ubiquitination in the Nedd4-2-depleted DRG neurons; by contrast, more TrkA was ubiquitinated upon NGF treatment (Figure R10).

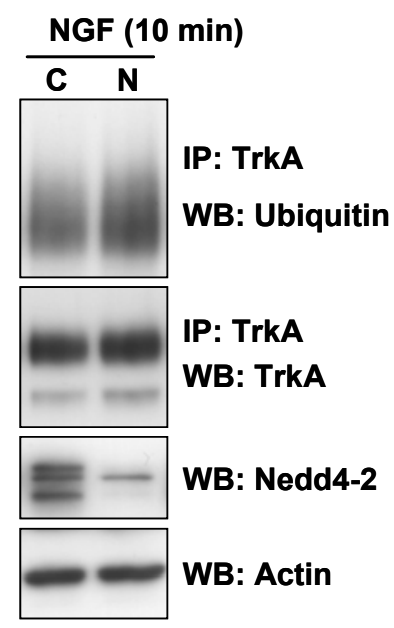

Figure R10. TrkA ubiquitination in response to NGF in rat DRGs

Infected DRG neurons were NGF stimulated for 10 minutes and immunoprecipitated with TrkA antibodies. C: Control shRNA lentivirus; N: Nedd4-2 shRNA lentivirus.

We next tested whether Nedd4-2 was able to ubiquitinate TrkA protein in different endosomal compartments. First, we tested the localization of Nedd4-2 by immunofluorescence using Nedd4-2 antibodies previously generated (Arevalo et al. 2006b). The specificity of Nedd4-2 antibody was confirmed with the lack of Nedd4-2 staining in Nedd4-2-depleted neurons as compared with control neurons (Figure R11A). Staining of NGF-dependent DRG neurons indicated a strong co-localization of Nedd4-2 with the early endosomal markers, EEA1 and Rab5, and with the late endosomal marker, Rab7 (Figure R11B). This localization resembles the localization of Itch, another member of the Nedd4 family (Marchese et al. 2003; Angers et al. 2004). 
A

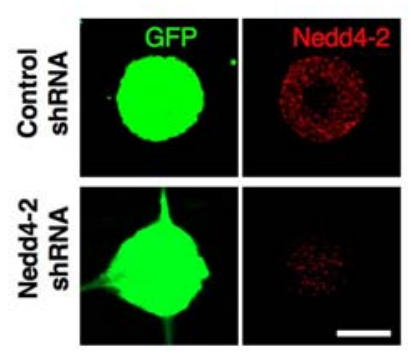

B

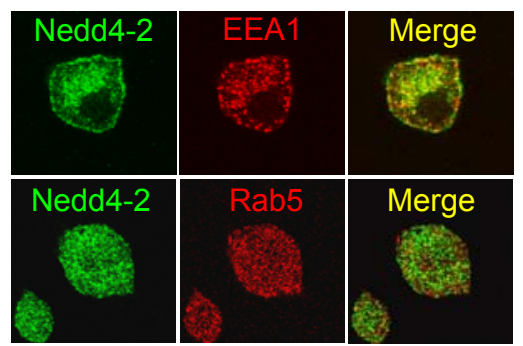

\begin{tabular}{c|c|c} 
Nedd4-2 & Rab7 & Merge \\
Nys & & \\
& & \\
\end{tabular}

Figure R11. Nedd4-2 localization in the early endosomes and late endosomes in sensory neurons

(A) Nedd4-2 antibody specificity. Nedd4-2 antibodies stain Nedd4-2 specifically. DRG neurons infected with control and Nedd4-2 shRNA lentivirus were stained with Nedd4-2 antibodies. Images from control and Nedd4-2 depleted neurons were acquired with a confocal microscope using the same settings and processed in a similar way. Note the almost complete disappearance of the signal in Nedd4-2-depleted neurons. Scale bar, $20 \mu \mathrm{m}$.

(B) Co-localization of Nedd4-2 with early endosomes (EEA1 and Rab5) and late endosomes (Rab7). Scale bar, $20 \mu \mathrm{m}$.

The localization of Nedd4-2 in endosomes led us to hypothesize that Nedd4-2 may play a role in the ubiquitination of TrkA in these compartments. Therefore, we next tested whether Nedd4-2 depletion specifically altered the ubiquitination status of TrkA in early endosomes. Overnight-starved DRG neurons infected with control and Nedd4-2 shRNA lentivirus were treated with NGF for 10 and 30 minutes to activate TrkA and this allowed the receptors to be ubiquitinated. Early endosomes were enriched using Rab5 antibody to pull them down after the neurons had been homogenized. Upon solubilization of the early endosomes, TrkA was immunoprecipitated and the samples were subjected to Western blot to test TrkA ubiquitination. The results showed that TrkA ubiquitination decreased in the early endosomes from Nedd4-2-depleted neurons (Figure R12), which indicated that Nedd4-2 regulates TrkA ubiquitination in the early endosomes. These data further 
supported the role of Nedd4-2-mediated ubiquitination of TrkA in its trafficking in these compartments.

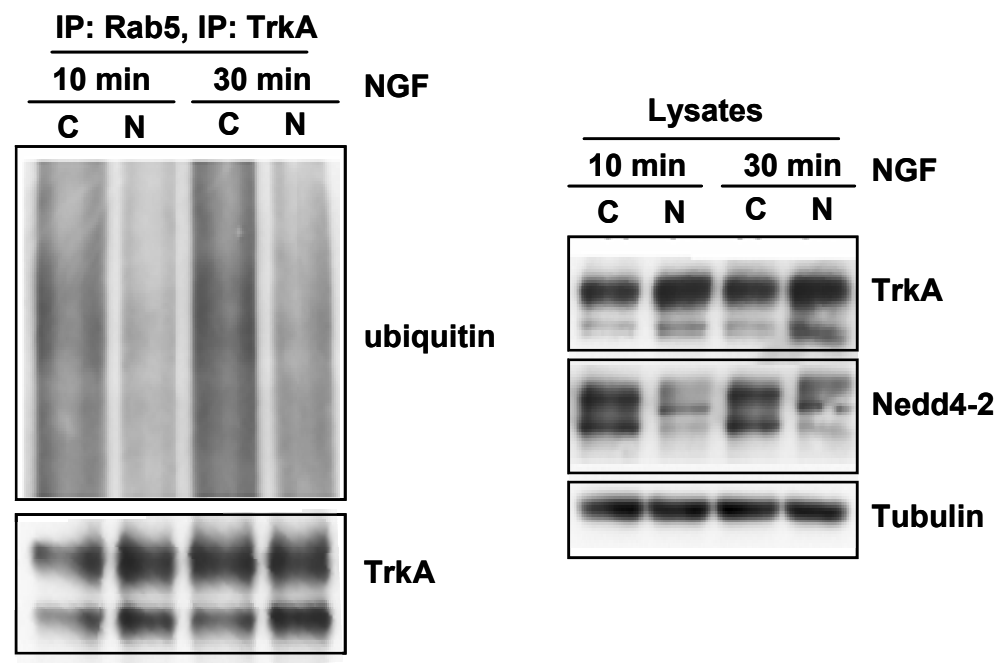

Figure R12. Reduced ubiquitination of activated TrkA in early endosomal compartments

Infected DRG neurons were homogenized, immunoprecipitated with Rab5 antibodies, and then immunoprecipitated with TrkA antibodies after solubilization. Representative blots are shown $(n=2)$. C: Control shRNA lentivirus; N: Nedd4-2 shRNA lentivirus.

\subsubsection{Nedd4-2 depletion increases TrkA signalling in rat DRG neurons}

From the results presented above we hypothesized that if the down-regulation of Nedd4-2 caused impaired degradation of activated TrkA and led to an increased surface level of TrkA, there could consequently be an increased downstream signalling induced by NGF. To address this issue, we infected NGF-dependent DRG neurons with control and Nedd4-2 shRNA lentivirus and performed western blot analyses in response to NGF. Infected neurons were NGF-starved overnight in the presence of the anti-apoptotic inhibitor Z-VAD-FMK, after which NGF was applied for different times $(0,5,15,30,45,60$ min). Cell lysates were collected and analyzed by western blot. Activation of TrkA and several signalling pathways downstream from TrkA was tested using antibodies that recognized activated forms of TrkA, PLCY, Akt and MAPK in order to assess the role of Nedd4-2. The levels of Nedd4-2 and $\beta$-tubulin were examined for the efficiency of 
infection and sample loading, respectively. Our results showed that the depletion of Nedd4-2 resulted in an enhancement of the phosphorylation of TrkA and, subsequently, the PLCY, Akt and MAPK signalling pathways (Figure R13). Thus, these data demonstrated that Nedd4-2 regulates NGF-mediated signalling.

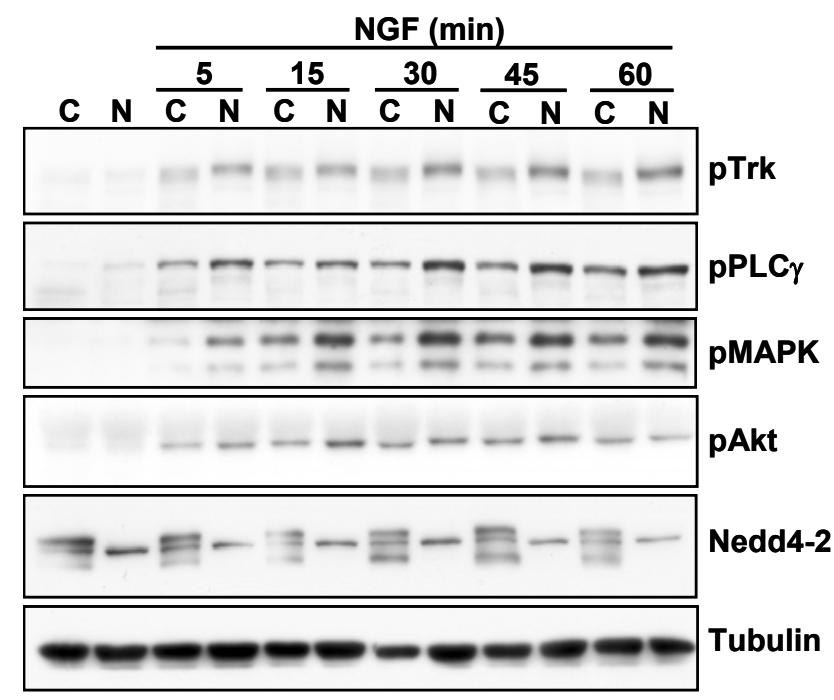

Figure R13. Nedd4-2 modulates NGF-mediated signalling.

Nedd4-2 depletion enhances NGF-mediated signalling. DRG neurons were infected with control and Nedd4-2 shRNA lentivirus, and cell extracts were analyzed upon NGF treatment. Active TrkA, PLCY, MAPK and Akt were assessed using antibodies that recognized specific phosphorylated residues. Representative blots are shown $(n=5)$. C: Control shRNA lentivirus; N: Nedd4-2 shRNA lentivirus.

\subsubsection{Nedd4-2 depletion enhances the retrograde transport of TrkA in DRG neurons}

Unlike other cell types, sensory neurons are characterized by their long axons, which in vivo reach the target area far away from where the cell body is located. An additional fate of internalized TrkA protein that escapes degradation is retrograde transport to the cell body (Zweifel et al. 2005). To address whether the depletion of Nedd4-2 affected the retrograde transport of activated TrkA, we set up compartmentalized DRG cultures using microfluidic compartments. Using this small device, we were able to accomplish the separation of the soma and the axon of the DRG neurons (Figure R14A). DRG neurons growing on microfluidic compartments were infected with control or Nedd4-2 shRNA lentivirus on DIV4, NGF-starved overnight at DIV7, and then stimulated with NGF at the 
distal axons for 15 hours. The rate of retrograde transport in the neurons was monitored by the presence of red fluorescent beads in the cell body compartment that were applied to the distal compartment (Figure R14B). Nedd4-2 depletion resulted in an increased amount of activated TrkA in the cell body compartment as compared with control neurons (Figure R14C). Therefore, the retrograde transport of activated TrkA is also affected by Nedd4-2 protein.

A

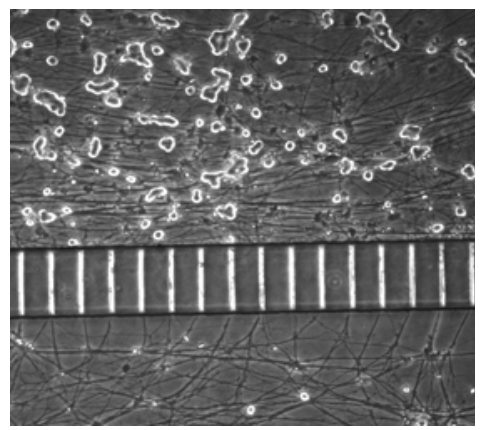

B

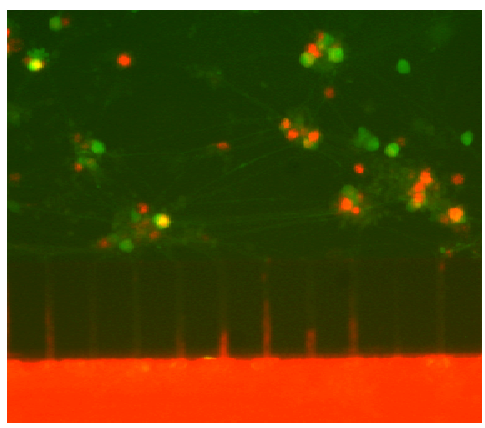

C

\begin{tabular}{|c|c|c|c|c|}
\hline C & $\mathbf{N}$ & C & $\mathbf{N}$ & Lentivirus \\
\hline- & - & + & + & NGF to Distal Axon \\
\hline+ & + & - & - & NGF to Cell Body \\
\hline & $2=$ & & $=$ & pTrk \\
\hline 100 & 90 & 23 & 74 & \\
\hline$=$ & $=$ & $=$ & & Tubulin \\
\hline
\end{tabular}

Figure R14. Enhanced NGF-mediated retrograde transport of pTrkA in Nedd4-2 depleted DRG neurons

(A) DRG neurons growing in microfluidic chambers. The cell body compartment (the upper part) and the axon compartment (the lower part) are clearly separated.

(B) Retrograde transport in infected neurons. The retrograde transport in the infected neurons (GFP-positive) was monitored by the presence of red fluorescence in the cell body compartment. Red-fluorescent beads were added to the distal compartment 15 hours before subsequent experiments were performed. Scale bar, $20 \mu \mathrm{m}$.

(C) Increased retrograde transport of pTrkA upon Nedd4-2 depletion. Neurons were infected with control or Nedd4-2 shRNA lentivirus and either not stimulated or stimulated with NGF for $10 \mathrm{~min}$ in the cell body compartment or for 15 hours in the distal compartment. Lysates were analyzed by western blot with pTrkA antibodies. Tubulin was used as a loading control. Numbers represent pTrk intensity normalized with respect to tubulin loading. C: Control shRNA lentivirus; N: Nedd4-2 shRNA lentivirus. A representative experiment is shown. 


\subsubsection{Nedd4-2 depletion increases the survival of NGF-dependent DRG neurons}

What are the functional consequences of Nedd4-2 depletion on DRG neurons? It is known that DRG neurons depend on neurotrophins through Trk-mediated signalling for their survival during development (Snider 1994). To address whether Nedd4-2 modulates the neurotrophin-dependence survival of sensory neurons, we obtained NGF- and BDNF-dependent neurons and knocked down their levels of Nedd4-2. The dissected neurons were transfected on DIV4-5 with plasmids that expressed GFP and control shRNA or GFP and Nedd4-2 shRNA. The presence of GFP allows transfected neurons to be identified unambiguously. Apoptotic and non-apoptotic cells were scored at $72-120 \mathrm{~h}$ after NGF or BDNF withdrawal (Figure R15A). The percentage of apoptosis observed upon NGF withdrawal in the Nedd4-2-depleted neurons was only $34.9 \%$, which was significantly reduced in comparison with the $60.8 \%$ of control transfected neurons (Figure R15B). Upon BDNF deprivation, no differences in apoptosis between control (58.7\%) and Nedd4-2 shRNA (58.6\%) transfected cells were observed in BDNF-dependent neurons (Figure R15B). Therefore, these data indicate that Nedd4-2 is able to specifically influence the survival of NGF-dependent sensory neurons through the modulation of NGF-mediated signalling. 
A

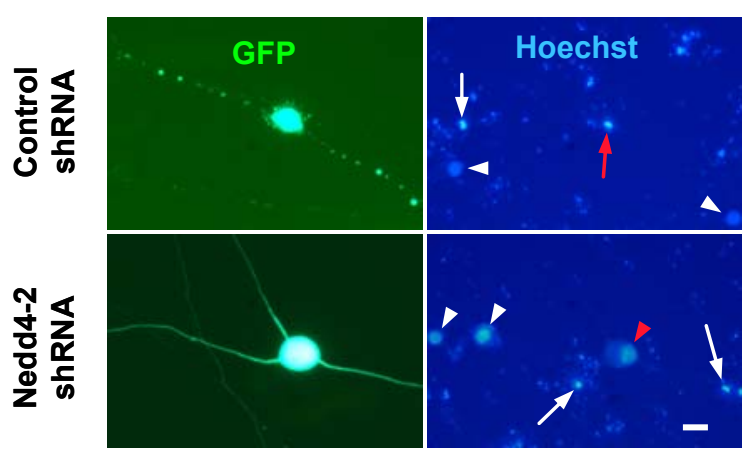

B

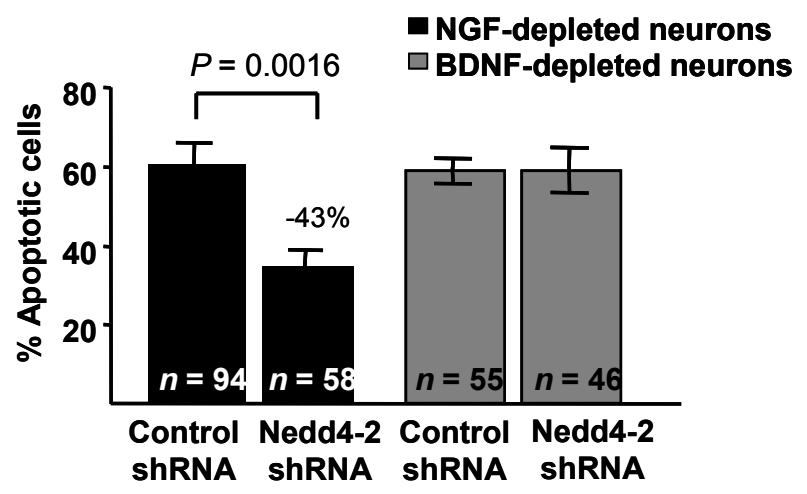

Figure R15. Nedd4-2 modulates NGF-mediated cell survival.

(A) Images showing NGF-dependent DRG neurons transfected with control and Nedd4-2 shRNA plasmids. Transfected cells were visualized by GFP expression, and non-apoptotic cells (arrowheads) and apoptotic cells (arrows) were identified by staining with Hoechst3342. Transfected neurons in the Hoechst3342 panel are indicated in red. Scale bar, $20 \mu \mathrm{m}$.

(B) Quantification of apoptosis in NGF- and BDNF-dependent neurons transfected with control and Nedd4-2 shRNA plasmids upon withdrawal of the corresponding neurotrophin for 72-120h. Data are presented as Mean \pm SEM. $P$ values were calculated using a two-tailed Student's t-test $[n=$ number of cells from 6 (NGF) and 3 (BDNF) independent experiments]. 


\subsection{Part two: Results from TrkAP782S mutant mice}

\subsubsection{Generation of a knock-in (KI) mouse model expressing TrkAP782S}

Nedd4-2 is an E3 ubiquitin ligase for several different substrates (Yang et al. 2010) and hence shRNA against Nedd4-2 could exert indirect effects on TrkA protein. Previously, it has been reported that the PPVY785 motif present in TrkA, but not in TrkB, is required for the binding of Nedd4-2 (Arevalo et al. 2006b). In order to rule out the possibility that our results in rat DRG neurons using Nedd4-2 shRNA lentivirus might be the consequence of an indirect effect, we mutated the PPVY785 motif of TrkA by replacing the first proline with a serine to obtain TrkAP782S, which would prevent the binding of Nedd4-2 to TrkA. TrkAP782S was assumed to be incapable of either binding to or being ubiquitinated by Nedd4-2. To confirm this, we transfected HEK293 cells with a Nedd4-2 plasmid together with a plasmid expressing WT TrkA or TrkAP782S to test the binding of Nedd4-2 to TrkAP782S and the ubiquitination of TrkAP782S. The results revealed that TrkAP782S lost the ability to bind Nedd4-2 and, consequently, it was not ubiquitinated (Figure R16), confirming that Nedd4-2 is an E3 ubiquitin ligase for TrkA that leads to its ubiquitination through the PPXY motif. 


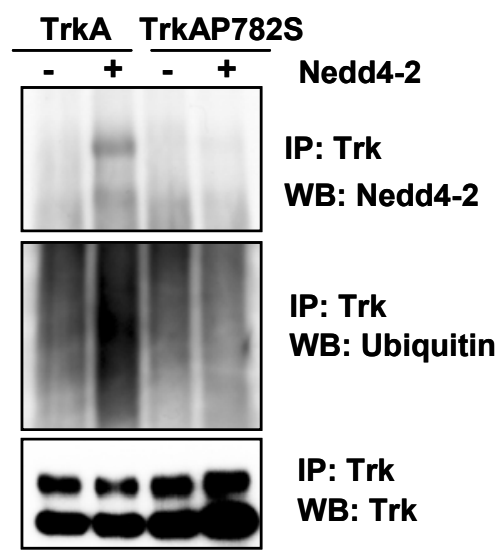

Figure R16. TrkAP782S can not be ubiquitinated by Nedd4-2

Nedd4-2 ubiquitinates and binds to wild-type TrkA but not TrkAP782S. Lysates from HEK293 cells transfected with TrkA or TrkAP782S receptor and FLAG-Nedd4-2 were immunoprecipitated using Trk antibodies. Western blots were performed to assess Nedd4-2 co-immunoprecipitation, TrkA ubiquitination and TrkA levels.

Based on these in vitro data, our laboratory, in collaboration with Dr. Lino Tessarollo (National Cancer Institute, Bethesda), generated a TrkAP782S mutant knock-in (KI) mouse. This mouse carries a point mutation in the TrkA DNA sequence in the codon that encodes proline 782 . Thus, proline 782 was replaced by serine, the amino acid present in TrkB. The targeting vector to generate the TrkAP782S mouse was constructed by introducing two changes in the proline 782 codon to code for serine in exon 17 (Figure $\mathrm{R} 17 \mathrm{~A})$. The presence of these changes was confirmed by sequencing and by the disappearance of a BstXI restriction site. Targeted ES cells were monitored by southern blotting with an external probe (Figure R17B) and with an internal (neo) probe (data not shown), as described in Material and Methods. KI mice were generated from targeted ES cells and they were viable and fertile, with a normal life span. The genotyping of the mice was performed by PCR, amplifying 1185 bp and 1385 bp fragments from the WT and KI, respectively (Figure R17C). The presence of the mutation was assessed by the lack of digestion of the PCR fragment with BstXI in the KI mice (Figure R17D). To confirm that the mutant amplified DNA could be digested, we used the Pstl restriction site (Figure R17D). 
A

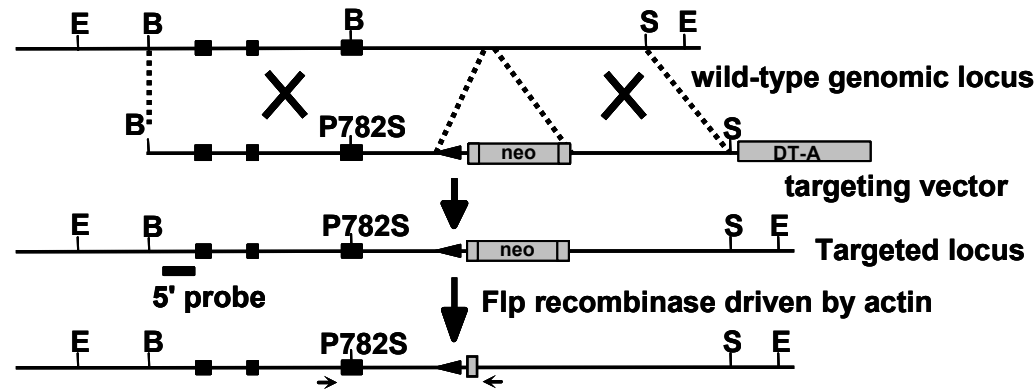

B

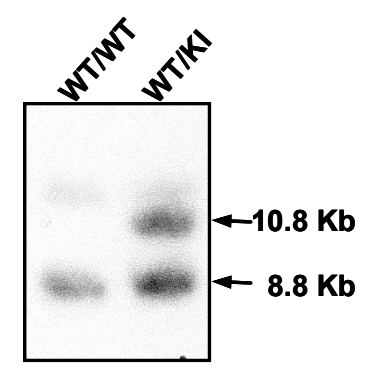

C
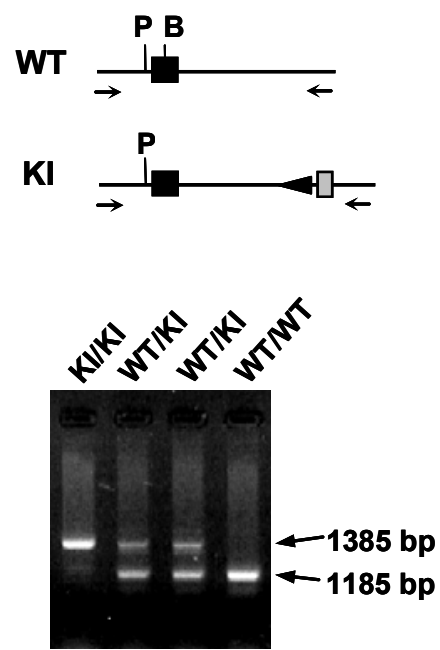

D

\begin{tabular}{c|c|c|c|} 
& PCR fragment & BstXI digestion & Pstl digestion \\
\hline WT & 1185 & $1016+169$ & $1041+144$ \\
\hline KI & 1385 & 1385 & $1241+144$ \\
\hline
\end{tabular}

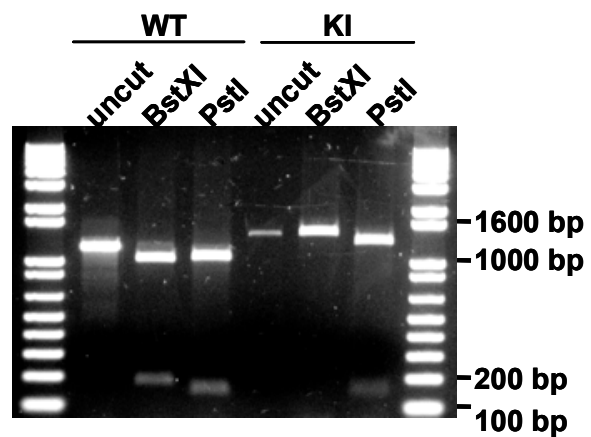

\section{Figure R17. Generation of TrkAP782S mutant mice}

(A) Schematics of the wild-type TrkA allele, the targeting vector, and the targeted allele before and after Flp recombination. Neo, neomycin resistance gene; DT-A, diphtheria toxin. E, B, and S denote the sites for the restriction enzymes EcoRV, BstXI, and Smal; black rectangles denote exons; triangles denote loxP sites; and grey rectangles flanking the neo cassette denote FRT sites.

(B) Southern blot analysis of genomic DNA from WT and targeted ES cells after digestion with EcoRV. As indicated in the schematics in (A), the $5^{\prime}$ probe detects an 8.8-kb fragment from WT alleles, and a 10.8-kb fragment from the targeted ES cells.

(C) PCR genotyping using mouse tail DNAs is shown. The primer sites used for genotyping are shown (arrows). B: BstXI; P: Pstl.

(D) The presence of the mutation that changes the proline to serine was assessed by BstXI digestion in the PCR product obtained from the KI animals. The upper panel shows the expected DNA fragments from digestion of the amplified DNA from the WT and KI mice. Note the lack of digestion with BstXI, but not with $P s t l$, in the amplified DNA fragment from the KI mouse. 


\subsubsection{Reduced amounts of TrkAP782S and TrkAP782S mRNA in KI DRGs}

Depletion of Nedd4-2 in rat DRGs afforded increased amounts of TrkA (Figure R2). To address the levels of TrkA in DRGs from KI mice, we obtained DRG neurons from WT and KI E13.5 embryos and cultured them in the presence of NGF to select TrkA-expressing neurons. To our surprise, we found that total TrkA levels were decreased in the KI mice as compared with those seen in WT mice (Figure R18A). To further confirm this, two different antibodies were used for TrkA detection in order to check the possibility that the mutation of TrkAP782S may cause some defect in the antibody recognition. It is known that C-14 antibody recognizes the cytoplasmic part of TrkA, while RTA antibody binds to the extracellular sequence of TrkA. We found a $32 \%$ and $23 \%$ reduction in TrkA levels with the C-14 and RTA antibodies, respectively (Figure R18B). These data suggest that the decrease in TrkA levels in the KI DRG neurons is not a consequence of the altered binding affinity of $\mathrm{C}-14$ antibody. Therefore, $\mathrm{KI}$ neurons express lower amounts of TrkAP782S protein.

A

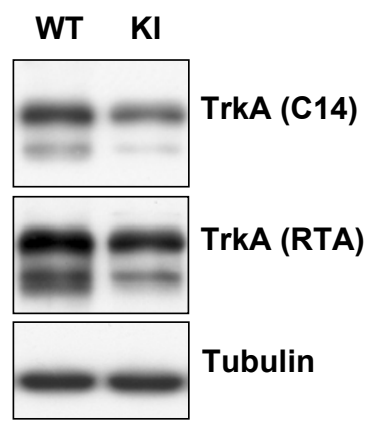

B

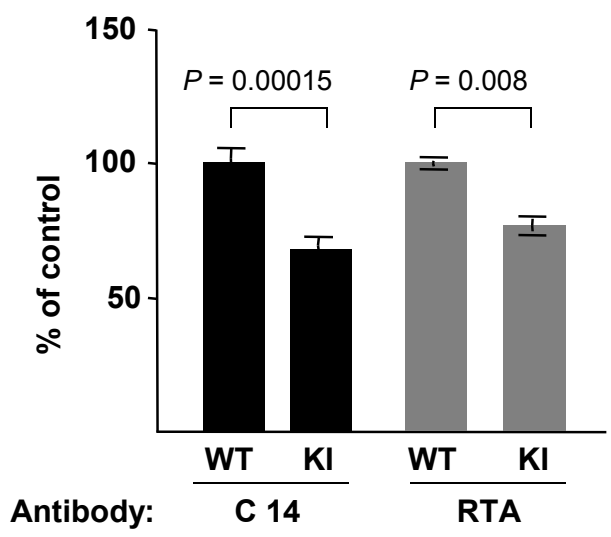

Figure R18. Reduced TrkA levels in the DRGs of KI mice

(A) Reduced amount of TrkAP782S protein in KI mice. Lysates from cultured DRG neurons were subjected to western blot analysis with the indicated antibodies.

(B) Quantification of TrkA expression in WT and KI DRG neurons. Data are presented as means \pm SEM. $P$ values were calculated using a two-tailed Student's t-test $(n=4)$. 
The level of a given protein is determined by its synthesis and degradation. Any alteration in these two processes may result in changes in the levels of the protein. To elucidate the cause of the decreased TrkAP782S levels in the KI mice, we measured the mRNA levels of TrkA by RT-qPCR using RNA extracted from E13.5 WT and KI DRG neurons in collaboration with Dr. Raquel Rodríguez's lab. The results showed that the mRNA level of TrkAP782S was significantly decreased as compared with TrkA in DRGs (Figure R19A), suggesting a potential cause for the reduced TrkAP782S protein levels in KI DRGs. Interestingly, when we normalized TrkA protein levels to the corresponding mRNA, the data showed that the decrease in TrkAP782S at protein level (23\%, Figure R18) was less prominent than that at transcriptional level (40\%, Figure R19A), suggesting that the degradation of TrkAP782S may be slower than TrkA (Figure R19B). Taken together, the results pointing to the decreased TrkAP782S levels are probably due to a reduced amount of mRNA synthesis.

A

TrkA

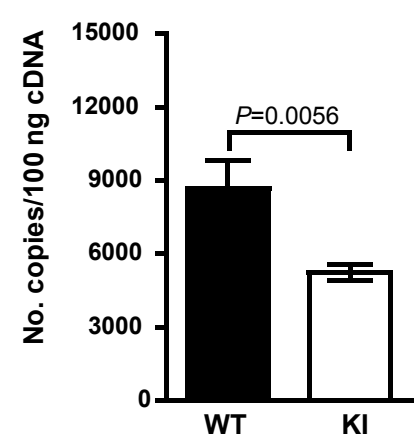

B-Actin

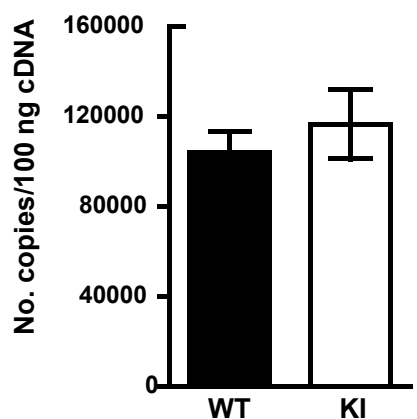

B

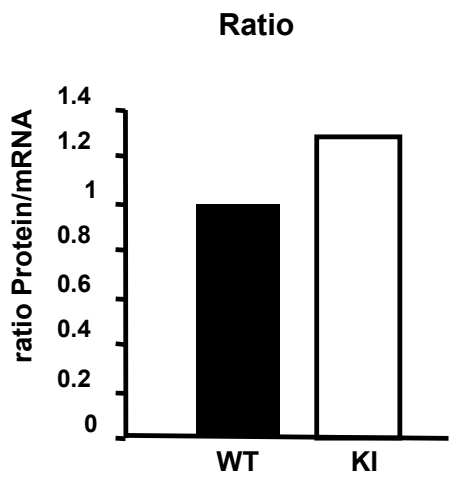

Figure R19. qPCR analysis shows a reduced expression of TrkAP782S mRNA levels in KI mice.

(A) mRNA extracted from the DRGs of E13.5 embryos from WT and KI mice were used for reverse transcription, followed by qPCR. The number of TrkA transcripts are shown as means \pm SEM $(n=2)$.

(B) Protein/mRNA ratio. 


\subsubsection{Impaired degradation of TrkAP782S in response to NGF treatment}

Based on the fact that with less mRNA expression in TrkAP782S neurons there is a greater relative amount of protein, we surmised that TrkAP782S may be more resistant to degradation than TrkA in response to NGF. Moreover, this idea is supported by previous findings such as the fact that the over-expression of Nedd4-2 decreased the levels of TrkA (Arevalo et al. 2006b) and the depletion of Nedd4-2 in sensory neurons resulted in a slower degradation rate of TrkA (Yu et al. 2011). The degradation rates of surface TrkAP782S and TrkA upon NGF treatment were measured in the WT and KI DRGs using biotinylation assays (Figure R20A). We observed a decrease in biotin-labeled WT TrkA levels upon NGF treatment for one hour in the WT DRG neurons, a consequence of the above degradation (Figure R20B, C). However, the difference in surface TrkAP782S levels before and after NGF treatment was indistinguishable in the KI DRG neurons (Figure R20B, C). No significant changes in p75 levels were observed upon NGF treatment in either WT or KI DRG neurons (Figure R20D). These data suggested that the degradation rate of TrkAP782S was slower than TrkA, similar to what we observed in the rat DRGs depleted of Nedd4-2 (Figure R3). 
A

Biotinylation

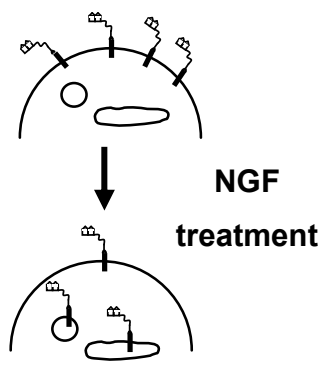

Surface-labeled TrkA

(not degraded)

C

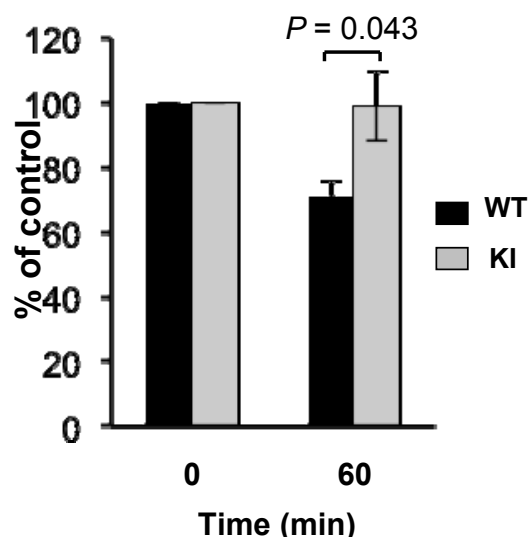

B

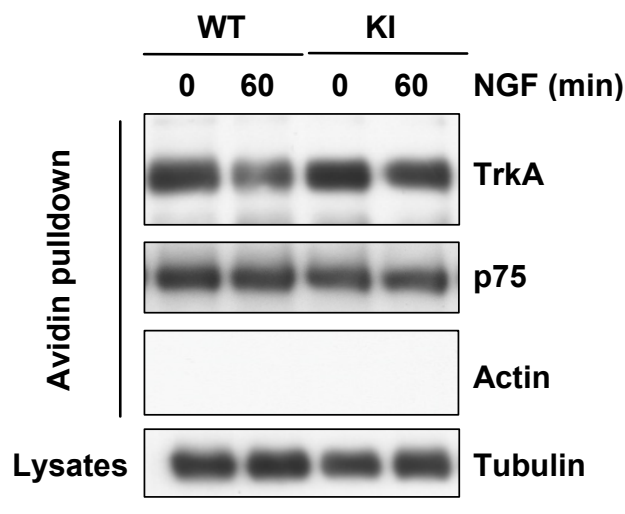

D

p75

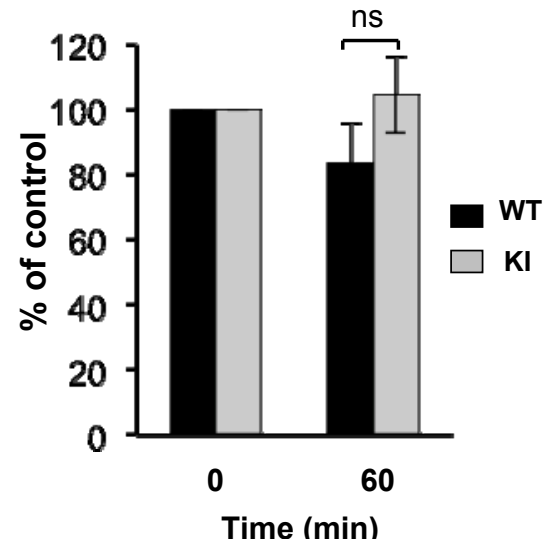

Figure R20. TrkAP782S and TrkA degradation in response to NGF in mouse DRGs

(A) A receptor degradation assay was performed as described in Material and Methods and is depicted in the schematic diagram.

(B) Representative western blots are shown. Actin was used as a negative control for biotinylated proteins and tubulin was used as a loading control for lysates.

(C) Quantification of surface-labeled TrkA. Data are normalized to the amount of biotinylated TrkA in WT and in $\mathrm{KI}$ neurons without NGF treatment. Data are presented as means \pm SEM. $P$ values were calculated using a two-tailed Student's t-test $(n=4)$.

(D) Quantification of surface-labeled p75. Data are normalized in the same way as TrkA. Data are presented as means \pm SEM. $P$ values were calculated using a two-tailed Student's t-test $(n=4)$.

In the degradative pathway, activated TrkA is internalized into early endosomes and then transferred to late endosomes and lysosomes for degradation. To test whether this trafficking route of TrkAP782S is altered in DRG neurons, we applied 
immunofluorescence to test the colocalization of pTrkA with the early endosomal marker EEA1 or with the late endosomal marker Rab7 in the WT and KI DRG neurons upon NGF treatment. Unlike WT TrkA, more activated TrkAP782S was found in the early endosomes with NGF treatment for 30 minutes (Figure R21A, B), indicating an accumulation of activated TrkAP782S there. However, we failed to observe any increase in the colocalization of activated TrkAP782S with the late endosomal marker Rab7 upon NGF treatment; instead, a significantly decreased amount of activated TrkAP782S was found in the late endosomes (Figure R22A, B), suggesting that less TrkAP782S is transported from early endosomes to late endosomes for degradation.

A

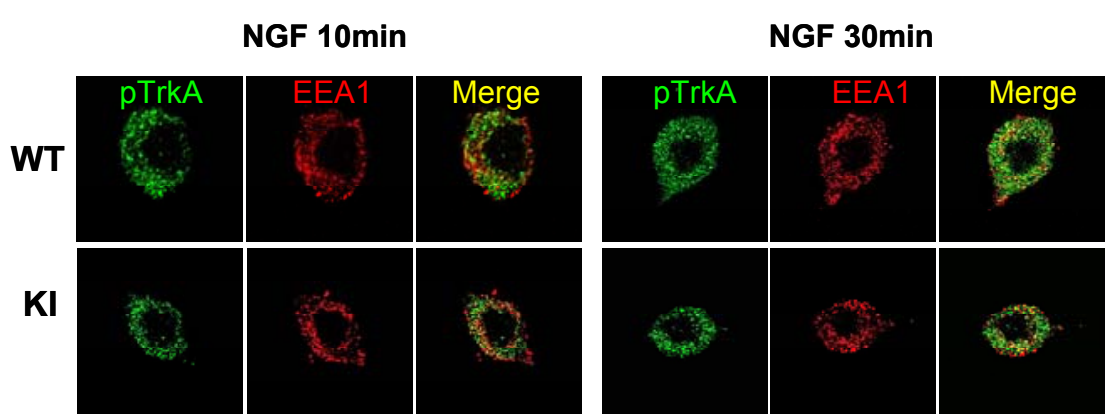

B

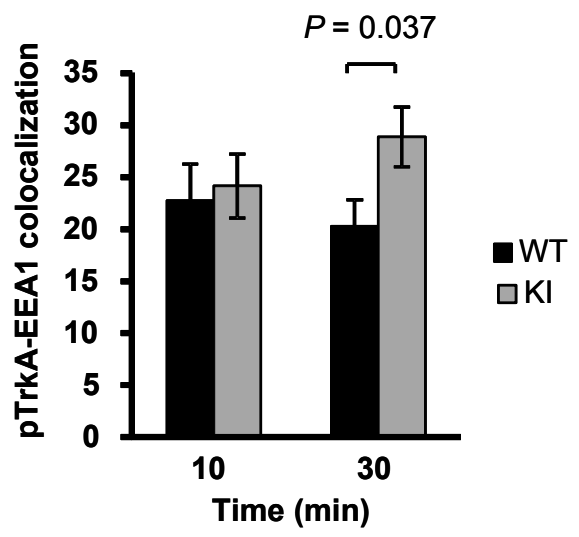

Figure R21. pTrkAP782S and pTrkA localization in early endosomes in mice DRGs

(A) Co-localization of pTrkA with EEA1 upon NGF treatment in WT and KI DRG neurons.

(B) Quantification of pTrkA co-localization in early endosomes. Data are presented as means \pm SEM. $P$ values were calculated using a two-tailed Student's t-test ( $n=5-7$ neurons/time-point). 
A

NGF 10min

NGF 30min
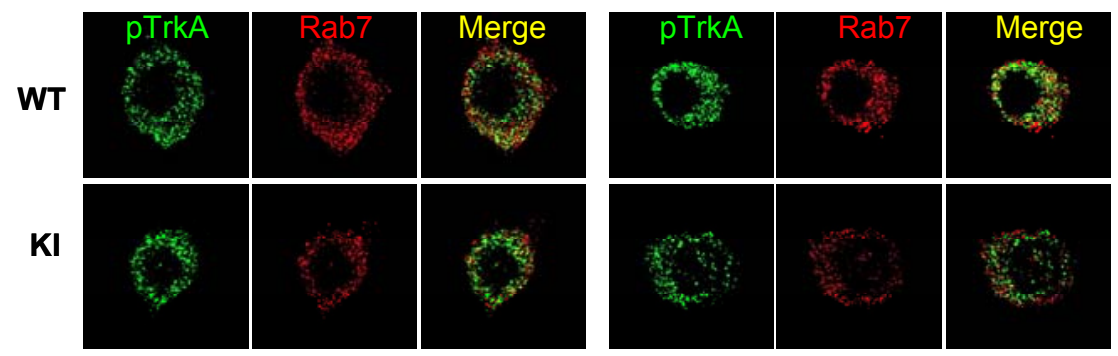

B

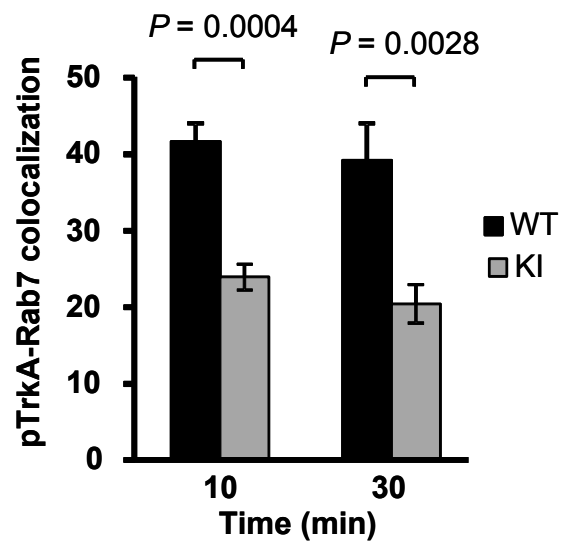

Figure R22. pTrkAP782S and pTrkA localization in late endosomes in mouse DRGs

(A) Co-localization of pTrkA with Rab7 upon NGF treatment in WT and KI DRG neurons.

(B) Quantification of pTrkA co-localization in late endosomes. Data are presented as means \pm SEM. $P$ values were calculated using a two-tailed Student's t-test ( $n=5-7$ neurons/time-point).

\subsubsection{Enhanced surface TrkA in DRG neurons from $\mathrm{KI}$ mice with NGF treatment}

As shown above, the degradation pathway of activated TrkAP782S is impaired compared with WT TrkA. To determine whether this would lead to an increased amount of TrkAP782S recycling, we tested the amount of TrkA at the cell surface at different time-points upon NGF treatment, as outlined in Figure R23A. We found that NGF treatment for one hour resulted in a clear reduction in surface WT TrkA in DRG neurons (Figure R23B). However, the reduction rate of surface TrkAP782S levels was much slower in DRG neurons (Figure R23B, C). No differences were detected in the decrease of surface p75 levels in response to NGF (Figure R23B, D). Additionally, when we tested the level of activated TrkA among the surface-TrkA neurotrophin receptors, we found that 
the level of activated TrkAP782S was similar or even higher than WT TrkA in DRG neurons with NGF treatment for one hour (Figure R23B). Together with the data from Arévalo et. al (Arevalo et al. 2006b) indicating that the internalization of TrkAP782S is not altered, these data revealed that the recycling of TrkAP782S is enhanced when Nedd4-2 does not bind to it, further supporting a potential role for Nedd4-2 in TrkA intracellular trafficking.

A

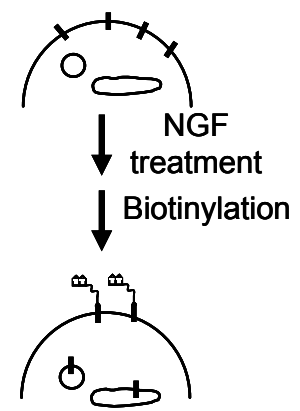

Surface-labeled proteins

C

TrkA

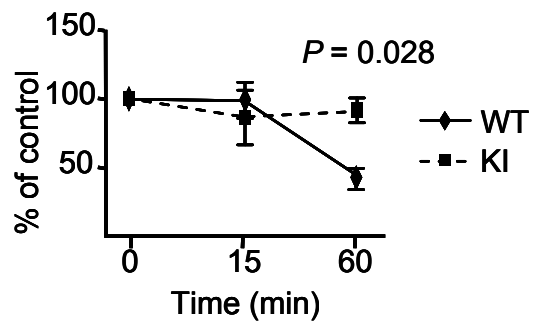

B

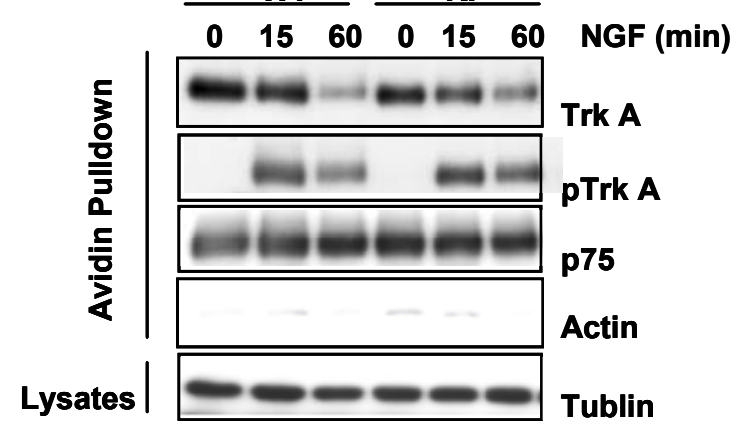

D p75

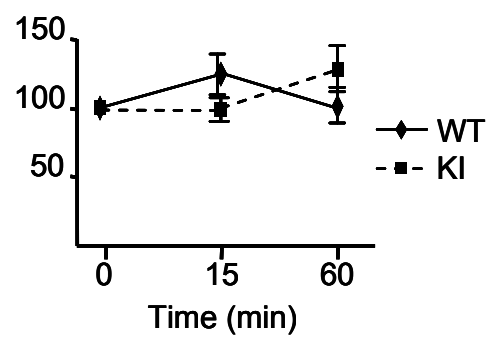

Figure R23. Enhanced surface pTrkA with NGF treatment in DRGs of KI mice

(A) A schematic diagram of the surface expression assay using the biotinylation procedure in response to NGF is shown.

(B) Representative western blots are shown. Cultured DRG neurons from E13.5 embryos of WT and KI mice were starved and treated with NGF $(50 \mathrm{ng} / \mathrm{ml})$ for 15 and 60 minutes and then biotinylated as described in Material and Methods. Cell lysates were prepared, labeled surface proteins were subjected to precipitation with neutroavidin-agarose, and western blot analyses were performed with the corresponding antibodies. Tubulin and actin were used as a loading control for lysates and as a negative control for biotinylated proteins, respectively.

(C) Quantification of surface TrkA upon NGF treatment. Data are presented as means \pm SEM. $P$ values were calculated using a two-tailed Student's t-test $(n=3)$.

(D) Quantification of surface p75 upon NGF treatment. Data are presented as means \pm SEM. $(n=3)$. 


\subsubsection{Decreased ubiquitination of TrkAP782S in the KI DRG neurons}

The ubiquitination of an internalized receptor determines its intracellular trafficking route (Grant et al. 2009; Grabbe et al. 2011). In order to find a possible reason for the enhanced TrkAP782S recycling, we next compared the ubiquitination level of TrkA with TrkAP782S upon NGF treatment in DRG neurons. We found that upon NGF treatment, TrkAP782S was less, but still, ubiquitinated than WT TrkA in the primary cultured DRG neurons (Figure R24), indicating that interruption of Nedd4-2 binding to TrkAP782S decreased TrkAP782S ubiquitination and hence may cause the alteration in TrkAP782S intracellular trafficking. At the same time, it also suggests that Nedd4-2 is not the only E3 ubiquitin ligase for TrkA in DRG neurons.

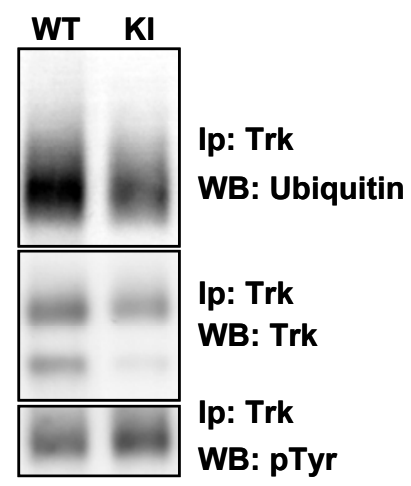

Figure R24. TrkAP782S is less ubiquitinated in vivo than wild-type TrkA.

Equal amount of lysates from WT and KI cultured DRG neurons treated with NGF $(50 \mathrm{ng} / \mathrm{ml})$ were immunoprecipitated with TrkA antibodies. Western blot analyses were performed to assess the levels of ubiquitination, expression and phosphorylation of TrkA proteins. A representative experiment is shown ( $n$ =3). WT: wild type TrkA; Kl: TrkAP782S.

\subsubsection{Enhanced TrkA signalling in DRG neurons from KI mice}

We have previously demonstrated that the down-regulation of Nedd4-2 levels led to enhanced surface-TrkA levels and an increase TrkA recycling in rat DRG neurons and, consequently, enhanced TrkA and its downstream signalling pathways upon NGF treatment. To address the NGF-mediated signalling emanating from TrkAP782S, DRG 
neurons from E13.5 WT and KI embryos were dissected and cultured as described previously. The results revealed that NGF-dependent neurons from KI mice showed an increase in TrkA, PLCy, Akt and MAPK activation after NGF treatment in comparison with that observed in WT DRG neurons, although the level of TrkAP782S was lower than that of TrkA (Figure R25). Therefore, the blockade of Nedd4-2 binding to TrkAP782S caused an increased TrkA activation and an increase in its downstream signalling pathways.

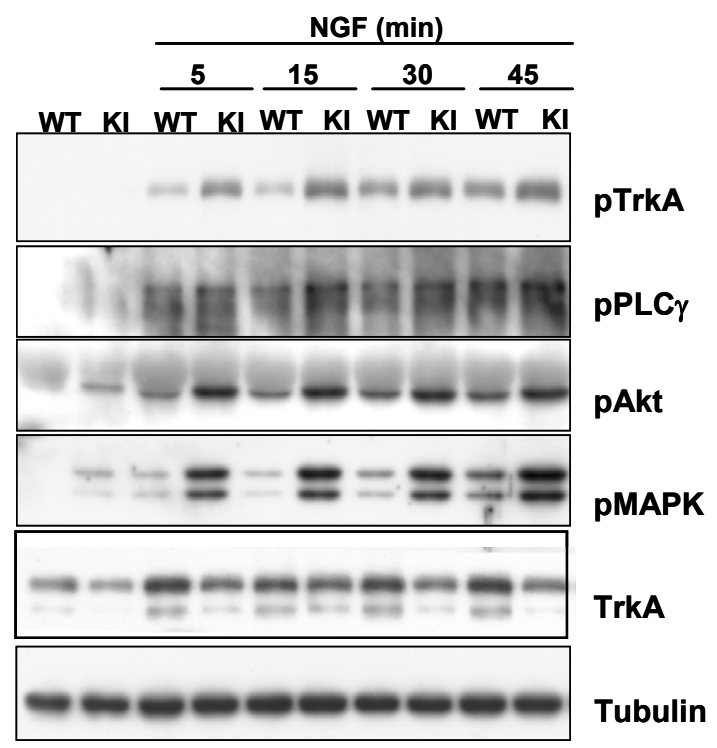

Figure R25. Enhanced NGF-mediated signalling in DRG neurons from KI mice

DRG neurons were obtained and cultured for 6-8 days, as described in Material and Methods. NGF was withdrawn and Z-VAD-FMK $(20 \mu \mathrm{M})$ was added to the neurons overnight. Stimulation with NGF $(50 \mathrm{ng} / \mathrm{ml})$ was performed for different times, and cell extracts were assessed using antibodies that recognized specific phosphorylated residues for TrkA, PLC $\gamma$, MAPK and Akt. Representative western blots are shown $(n=4)$. WT: wild-type TrkA; KI: TrkAP782S.

\subsubsection{Enhanced survival of DRG neurons in vitro and in vivo from KI mice}

To address the functional consequences of TrkAP782S expression in DRGs, we performed survival assays in NGF-dependent neurons obtained from WT and KI mice. Dissected neurons were plated with different amounts of NGF $(25,20,5,1,0 \mathrm{ng} / \mathrm{ml})$ and survival was monitored on DIV6-9. Without NGF application, the dissected neurons died on the second day after plating. We found that the survival of WT DRG neurons was 
related to the NGF concentration: the lower the concentration of NGF, the fewer living cells were observed. However, in the KI DRG neurons cell survival was not dependent on the NGF concentration: decreasing the amount of NGF did not lead to the death of such neurons (Figure R26). In particular, we found that KI neurons cultured with $1 \mathrm{ng} / \mathrm{ml}$ NGF survived as well as neurons cultured with $25 \mathrm{ng} / \mathrm{ml}$ NGF, while only $20.09 \%$ of WT neurons survived with $1 \mathrm{ng} / \mathrm{ml}$ NGF (Figure R26). Therefore, TrkAP782S confers a survival advantage to DRG neurons in limiting amounts of NGF, very probably due to the increased TrkA signalling.

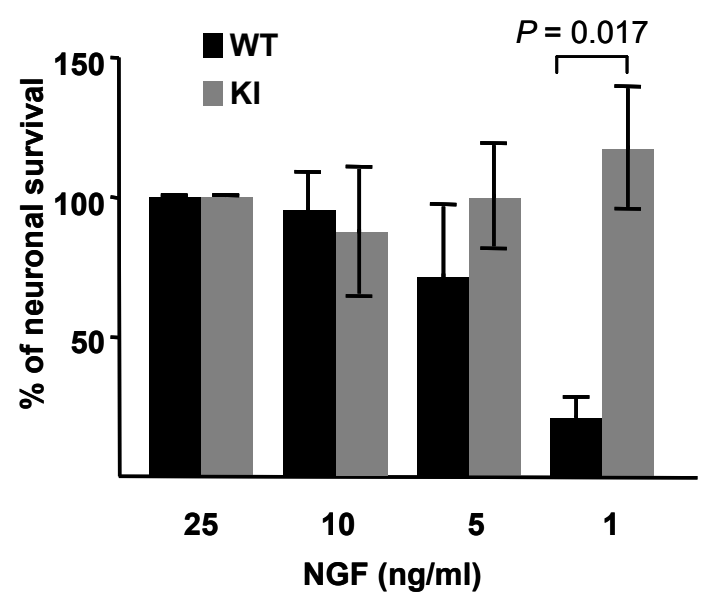

Figure R26. Increased survival of DRG neurons from KI mice with limiting amounts of NGF

DRG neurons from WT and KI embryos were plated on different amounts of NGF (25, 20, 10, 5, and 1 $\mathrm{ng} / \mathrm{ml}$ ). The number of live neurons present in medium supplemented with $25 \mathrm{ng} / \mathrm{ml}$ of NGF was considered $100 \%$ and the rest were normalized with respect to this percentage. Data are presented as means \pm SEM. $P$ values were calculated using a two-tailed Student's t-test $(n=5)$. WT: wild-type TrkA; KI: TrkAP782S.

During development, DRG neurons project to their targets and receive trophic support from the neurotrophins provided by the tissues innervated. Neurons that do not receive enough signalling will die (Snider 1994). In vitro survival experiments revealed that DRG neurons from $\mathrm{KI}$ mice survived better than the neurons from WT mice with limiting amounts of NGF in the culture (Figure R26). We next addressed whether the survival of DRG neurons in WT and KI was different during development. We counted the neurons in the L3 ganglia from WT and KI mice at P0 (Figure R27A) and found that the average 
number of neurons in the KI DRGs $(4578 \pm 276)$ was significantly increased compared to that in the WT DRGs $(3100 \pm 191)$ (Figure R27B). Therefore, the lack of Nedd4-2 binding to TrkAP782S leads to an increased cell survival in vitro and enhanced neuronal numbers in vivo.

A

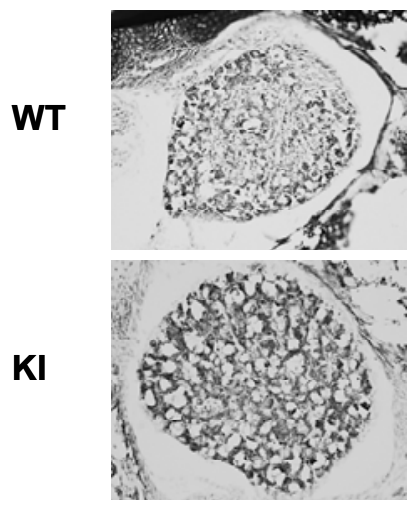

B

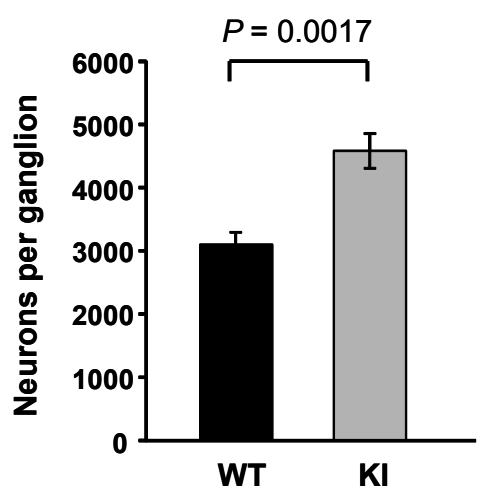

Figure R27. Number of neurons in L3 ganglia in WT and KI mice

(A) Cresyl violet staining of L3 Ganglion from WT and KI mice at P0.

(B) Quantification of the numbers of DRG neurons. Data are presented as means \pm SEM. $P$ values were calculated using a two-tailed Student's t-test $(n=4)$. WT: wild-type TrkA; KI: TrkAP782S.

\subsubsection{Enhanced sensitivity to acute noxious thermal stimuli in KI mice}

It has previously been reported that TrkA is expressed in the nociceptive small-diameter neurons in DRGs (Martin-Zanca et al. 1990) and that NGF-TrkA signalling is involved in nociception (Crowley et al. 1994; Smeyne et al. 1994; Vega et al. 1994; Ugolini et al. 2007). To test whether KI mice exhibited changes in their nociception, we performed tail flick and cold-plate assays to assess their threshold to acute noxious thermal stimuli. Using an automatic tail flick apparatus, the latency to remove the tail from the heat source was measured. We observed a significant decrease in withdrawal latency in the $\mathrm{KI}$ mice $(3.09 \pm 0.2 \mathrm{~s})$ as compared with WT mice $(5.8 \pm 0.1 \mathrm{~s})$ (Figure R28A), suggesting that KI mice are more sensitive to acute noxious heat stimuli. Then, we performed another thermal stimulus involving cold. WT and $\mathrm{KI}$ mice were placed in a container with the surface temperature at $0-4^{\circ} \mathrm{C}$. The time from when the mice were placed on the cold 
surface until they started licking/biting, lifting, shaking the hind paw or jumping was recorded. Similar to what we observed in the tail flick test, KI mice $(4 \pm 0.55 \mathrm{~s})$ presented an earlier response than WT mice $(6.33 \pm 0.58 \mathrm{~s})$ to the noxious cold stimulus (Figure $\mathrm{R} 28 \mathrm{~B})$. All these data indicate that $\mathrm{KI}$ mice are more sensitive to acute noxious thermal stimuli.

A

Tail Flick

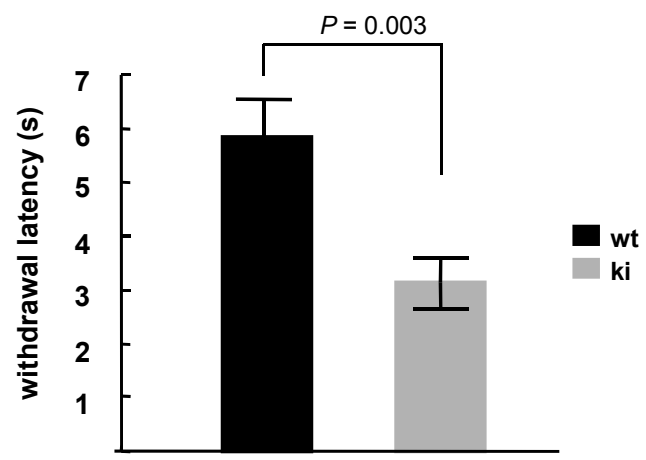

B

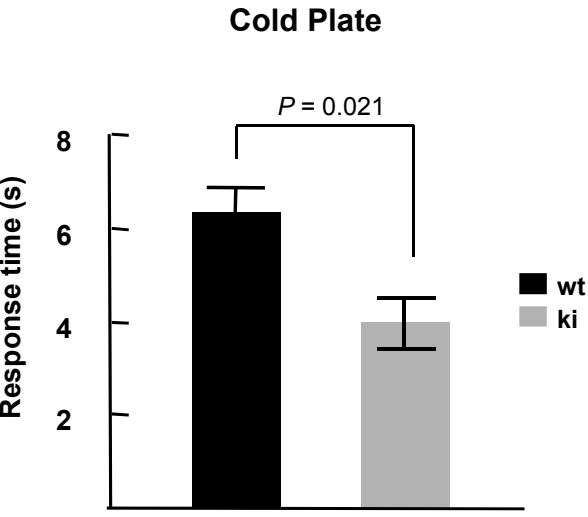

Figure R28. Response of WT and KI mice to acute noxious thermal stimuli

(A) Withdrawal latency of WT and KI mice to noxious heat. KI mice showed a significantly decreased latency. Data were expressed as means $\pm \mathrm{SEM}, P$ values were calculated using a two-tailed Student's t-test (WT mice $n=11, \mathrm{KI}$ mice $n=15$ ). WT: wild-type TrkA; KI: TrkAP782S.

(B) Withdrawal latency of WT and $\mathrm{KI}$ mice to noxious cold. Data were expressed as means $\pm \mathrm{SEM}$. $P$ values were calculated using a two-tailed Student's t-test (WT mice $n=9 ; \mathrm{KI}$ mice $n=5)$. WT: wild-type TrkA; KI: TrkAP782S.

\subsubsection{WT and KI mice respond similarly to acute noxious mechanical stimuli}

To test how KI mice respond to mechanical stimull, we subjected the WT and KI mice to the Randall-Selitto Test to measure the maximal pressure they were able to support on the paw. No significant differences were observed between the WT and KI mice in their withdrawal latency (Figure R29), indicating that the sensitivity in the KI mice for noxious mechanical stimuli was not altered. 


\section{Paw pressure}

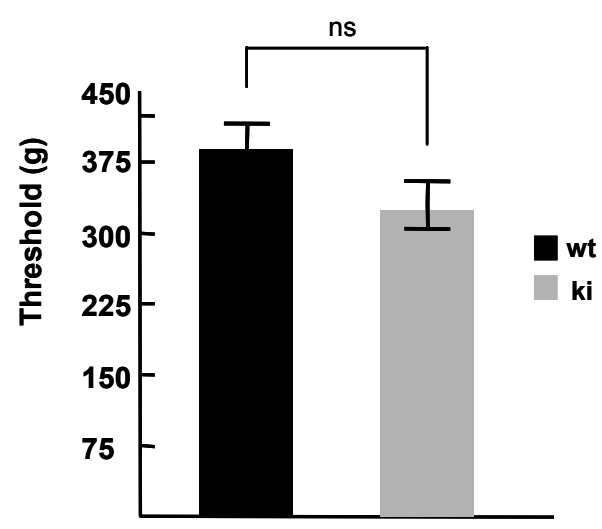

Figure R29. Responses of WT and KI mice to acute noxious mechanical stimulus Data were expressed as means \pm SEM, $P$ values were calculated using a two-tailed Student's t-test (WT mice $n=20 ; \mathrm{KI}$ mice $n=20$ ). WT: wild type TrkA; KI: TrkAP782S.

\subsubsection{Enhanced response of $\mathrm{KI}$ mice to formalin induced inflammatory pain}

It is widely documented that NGF acts as an inflammatory mediator (Pezet et al. 2001). After observing an increased TrkA signalling upon NGF treatment in the DRG neurons from KI mice, we hypothesized that KI mice may respond differently to inflammatory pain. To test this idea, we performed the formalin assay. Briefly, $20 \mu \mathrm{l}$ of $5 \%$ formalin was injected into the right hind paw of both the WT and the $\mathrm{KI}$ mice to induce chemical inflammation in the injected area, and the mice were recorded using a video camera for 55 minutes. Licking or biting of the injected paw was considered to reflect pain. The time each animal spent on these types of behaviour was counted every 5 minutes. We found that the $\mathrm{KI}$ mice spent a significantly increased time in licking or biting the formalin-injected paw than the WT mice, suggesting that they felt more pain (Figure R30A). Moreover, the responses of the mice to formalin injection was also analyzed by the presence of two different phases. Phase I, the acute phase (0-15 min), corresponded to the burning phase, whereas phase II, the chronic phase (15-55 min), corresponded to the inflammatory phase (Hunskaar et al. 1987). The KI mice spent more time licking/biting the formalin injected paw than the WT mice in both phases (WT mice: $51 \pm 7.65 \mathrm{~s} v \mathrm{~s}$. KI mice: $105.4 \pm 14.6$ s in phase I; WT mice: $205.9 \pm 43$ s vs. KI mice: $478.7 \pm 63$ s in phase II) 
(Figure R30B). Therefore, $\mathrm{KI}$ mice present a higher sensitivity to inflammatory pain than WT mice.

A Formalin Assay

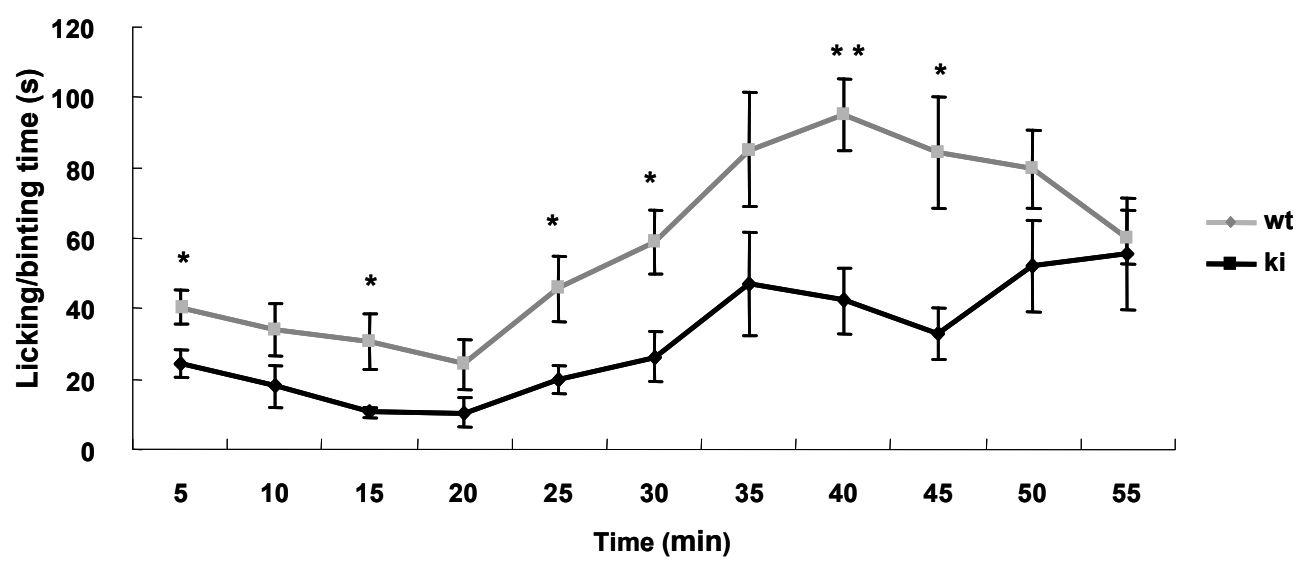

B

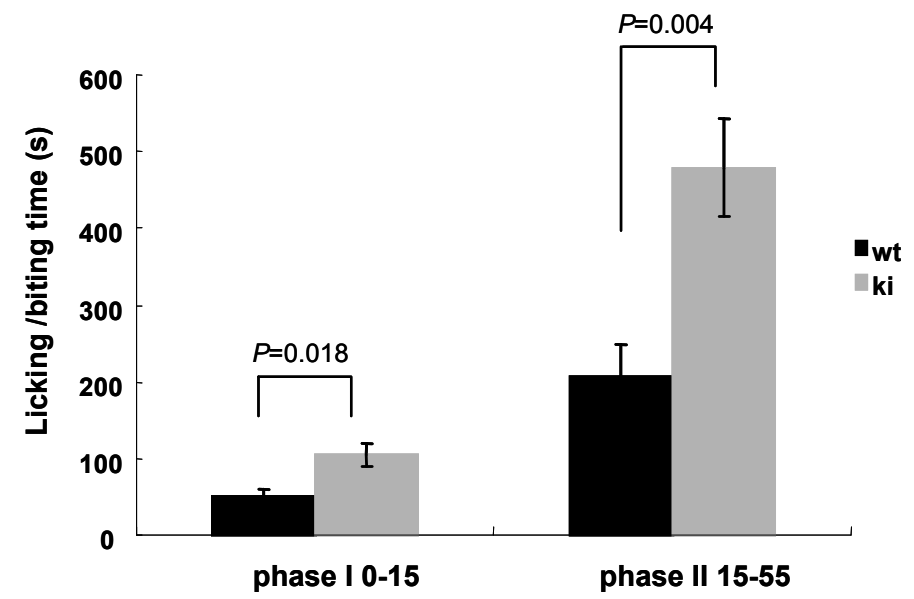

Figure R30. Response of WT and KI mice to noxious chemical stimulation

(A) The time spent in licking/biting the injected paw every 5 minute are shown for the total time-course (55 minutes after formalin injection). Data are expressed as means \pm SEM (WT mice $n=10$; KI mice $n$ $=13) .{ }^{*} p<0.05,{ }^{* *} \mathrm{P}<0.01$ (Mann-Whitney test).

(B) Total licking/biting time in phase I (0-15 minute after formalin injection) and phase II (15-55 minutes after formalin injection). Data are expressed as means \pm SEM. $P$ values were calculated using a two-tailed Student's t-test (WT mice $n=10, \mathrm{KI}$ mice $n=13$ ). WT: wild-type TrkA; KI: TrkAP782S.

The expression of c-fos has been described to increase rapidly in response to neuronal activity (Greenberg et al. 1987). To address the neuronal activity in the spinal cord of the WT and $\mathrm{KI}$ mice in the formalin assay, c-fos expression was tested. Two hours after the formalin injection into the hind paw, the animals were perfused with paraformaldehyde, 
and c-fos expression was assessed by immunohistochemistry. We observed an increased c-fos expression in the lumbar spinal cord of the ipsilateral side in the $\mathrm{KI}$ mice (Figure R31), consistent with the behavioural response to formalin injection in which the $\mathrm{KI}$ mice spent more time licking/biting the injected paw; however, no signal of c-fos was detected on the contralateral side (Figure R31). Therefore, the neurons in the spinal cord of the $\mathrm{KI}$ mice are more active than those in the WT mice after formalin injection, which may explain why $\mathrm{KI}$ mice are more sensitive to inflammation.

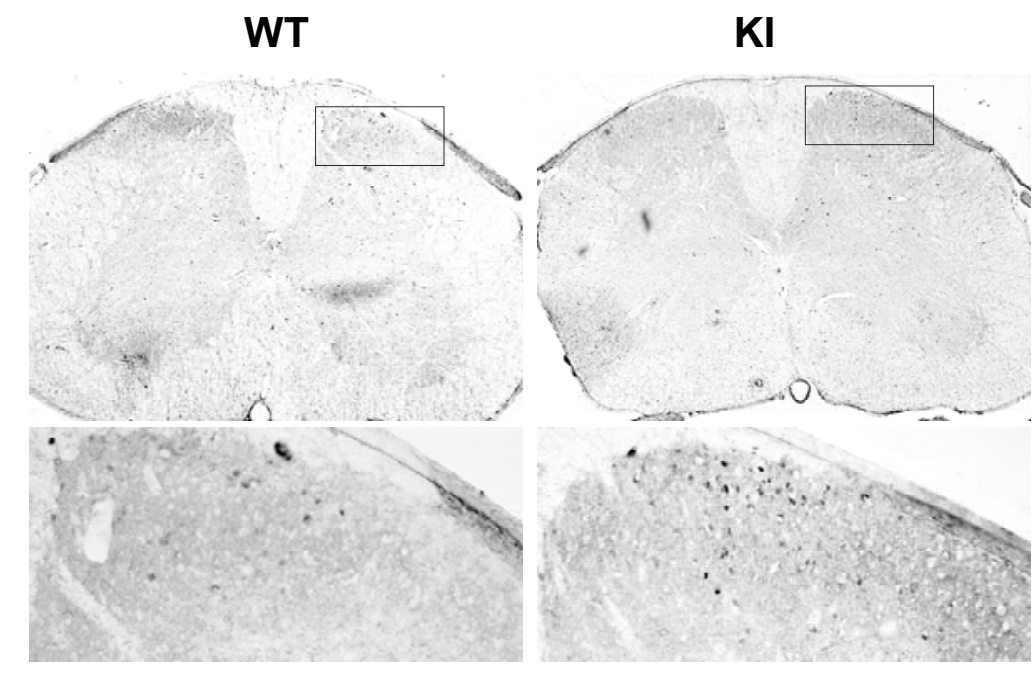

Figure R31. C-fos expression in the lumbar spinal cord in WT and KI mice after formalin injection

Immunohistochemistry using c-Fos antibody was performed in spinal cord sections two hours after formalin injection into the right hind paw. Top: C-fos staining was observed in the ipsilateral spinal cord dorsal horn. Bottom: c-fos staining at higher magnification. WT, wild-type mice; KI, TrkAP782S mutant mice.

\subsubsection{KI mice have a up-regulated mRNA levels of pain-related neuropeptides}

To address the mechanism of enhanced sensitivity in the $\mathrm{KI}$ mice, we focused on the neuropeptides SP and CGRP, because these are involved in the pain sensation to noxious stimuli, and at the same time are regulated by TrkA signalling (Harmar et al. 1980; 
Otten et al. 1980). We tested the levels of SP and CRGP mRNA in DRG from P0 WT and $\mathrm{KI}$ mice using in situ hybridization. The results showed a slight increase in CGRP and an evidently enhanced expression of SP in the DRGs of KI mice compared with WT mice (Figure R32), suggesting that $\mathrm{KI}$ mice are born with a higher expression of the neuropeptide SP--a major regulator in pain transmission. These results therefore offered a possible explanation for the lower threshold to acute thermal stimuli in the $\mathrm{KI}$ mice.

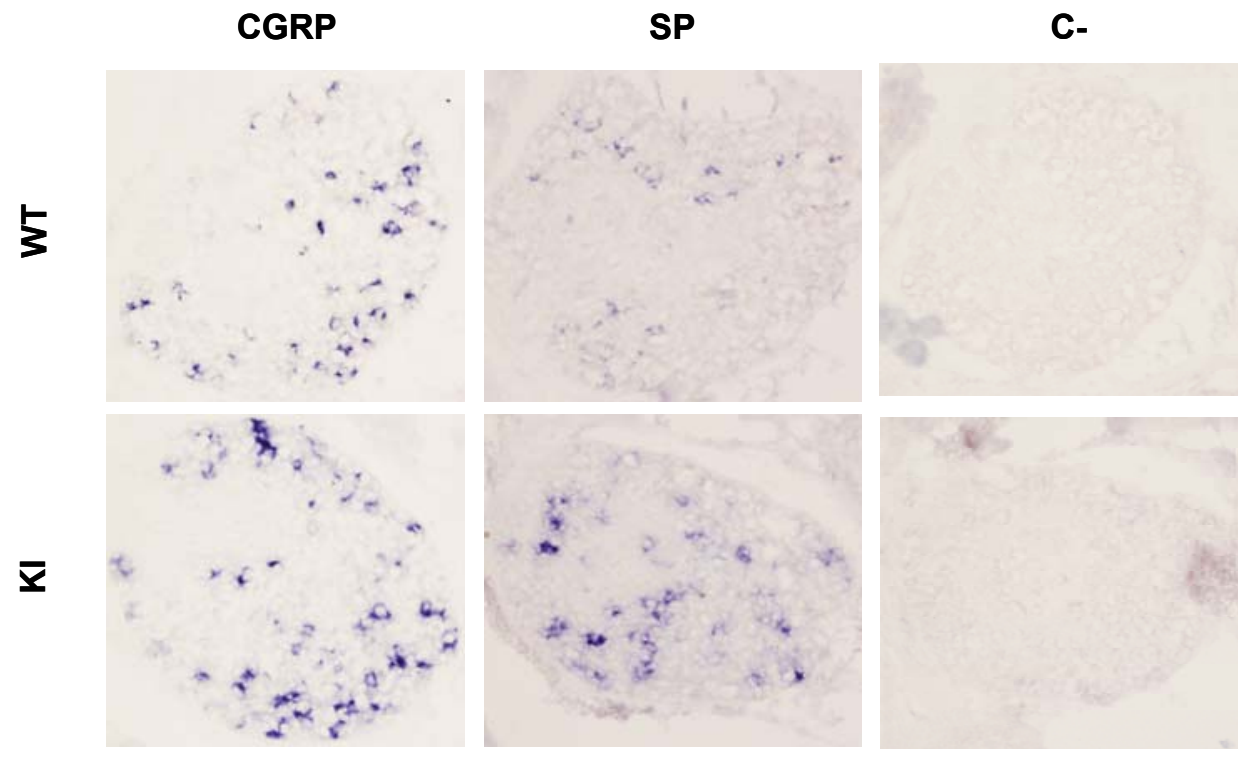

Figure R32. Expression of SP and CGRP in DRGs from WT and KI mice at P0

SP and CGRP were detected using in situ hybridization in DRGs from WT and KI mice at PO. New-born mice at P0 were fixed and secctioned. Stainings were compared between the same DRGs (L3-4) from the WT and KI mice. C-, control staining with sense probe for SP. 


\section{Discussion}


In the present work we provide evidence that the E3 ubiquitin ligase Nedd4-2 modulates NGF-mediated TrkA functions in vitro and in vivo via the modulation of TrkA ubiquitination and the regulation of TrkA intracellular trafficking. The depletion of Nedd4-2 in rat DRG neurons alters the intracellular sorting of activated TrkA by reducing the level of TrkA ubiquitination in early endosomes, which results in enhanced TrkA recycling and retrograde transport and decreased TrkA degradation. Consequently, TrkA signalling is increased in these neurons and so is their survival with limited amounts of NGF. The function of Nedd4-2 on TrkA is specific, since alterations in Nedd4-2 levels do not affect the neurotrophin receptor p75. The fact that Nedd4-2 regulates TrkA intracellular trafficking was further confirmed in DRG neurons from a $\mathrm{KI}$ mouse expressing a mutant TrkA protein that does not bind Nedd4-2. In addition, we observed that KI mice are more sensitive to acute noxious thermal stimuli and chronic inflammatory pain. These effects may be due to the increased amount of DRG neurons, an increased expression of SP in DRGs, and enhanced neuronal activity in the spinal cord upon stimulation. Taken together, TrkA ubiquitination mediated by Nedd4-2 plays an important role in NGF-mediated functions.

\subsection{Nedd4-2 regulates TrkA trafficking in the early and late endosomal compartments}

Since Nedd4-2 is an E3 ubiquitin ligase for TrkA (Arevalo et al. 2006b), we tested the role of Nedd4-2 in TrkA degradation. The results indicated that TrkA degradation in response to NGF treatment was much slower in the Nedd4-2-depleted DRG neurons than in control neurons (Figure R3). Therefore, Nedd4-2 depletion leads to a decreased degradation of TrkA in response to NGF treatment and this therefore results in increased amounts of total- and surface-TrkA in DRG neurons (Figure R2). Like other RTKs, TrkA undergoes intracellular trafficking before being degraded in lysosomes upon NGF treatment. In order to determine whether the slower TrkA degradation observed in Nedd4-2.-depleted DRG neurons was a consequence of some alteration in TrkA trafficking, we assessed TrkA 
intracellular transport in DRGs upon NGF treatment. We first tested whether Nedd4-2 depletion caused any change in the internalization of TrkA once it had been activated. On one hand, internalization is the initial step of intracellular trafficking of activated TrkA and any alteration in this step will affect the ensuing trafficking; on the other hand, ubiquitination of the activated receptor by an E3 ubiquitin ligase may be involved in regulating endocytosis (Terrell et al. 1998). Geetha et al. found that K63 poly-ubiquitination is required for TrkA internalization and signalling. They observed that TrkA is a substrate of TRAF6, and an absence of TRAF6 abrogated TrkA poly-ubiquitination and internalization (Geetha et al. 2005). However, in 2006 Arévalo et al. reported that TrkA can also be multi-monoubiquitinated by Nedd4-2 in an NGF-dependent manner, and that this ubiquitination did not have any effect on TrkA internalization (Arevalo et al. 2006b). Our data using immunofluorescence and biochemical experiments showed that the internalization of activated TrkA in Nedd4-2-depleted neurons is as efficient as in control neurons (Figure R4, R5). Therefore, the internalization of activated TrkA is not affected by the depletion of Nedd4-2 in rat DRG neurons. Together with the results from Geetha et al., these observations suggest that different forms of ubiquitination of TrkA may be involved in distinct biological functions. While the K63 poly-ubiquitination of TrkA may affect its internalization, the multi-monoubiquitination of TrkA caused by Nedd4-2 does not affect internalization and may have a role in intracellular trafficking.

Activated receptors in early endosomes following internalization can be transported either to late endosomes for degradation or to recycling endosomes to be reinserted back into the plasma membrane. Since both routes afford completely different outcomes, a small alteration in this process may result in distinct biological functions. To assess the trafficking of activated TrkA, we applied immunofluorescence staining of pTrkA together with the late endosomal marker Rab7 or with the recycling endosomal marker Rab11. Upon NGF treatment for 10 minutes, we observed less pTrkA in late endosomes (Figure R6), but more pTrkA in recycling endosomes (Figure R8) in Nedd4-2-depleted DRG neurons, indicating that Nedd4-2 depletion alters TrkA intracellular trafficking by 
transporting more TrkA to the recycling route. Interestingly, after NGF treatment for 30 minutes we found an increase in pTrkA levels in both the late endosomes and in the recycling endosomes in Nedd4-2-depleted DRG neurons (Figure R6, R8). These results suggest a hindered transport of pTrkA from late endosomes to lysosomes, in addition to an increased recycling of TrkA in these neurons. Therefore, Nedd4-2 must play an important role in the regulation of TrkA intracellular trafficking in the early and late endosomal compartments. The depletion of Nedd4-2 impaired TrkA degradation by diverting more TrkA to the recycling pathway, which was further confirmed by testing surface TrkA levels after NGF treatment of DRG neurons (Figure R9).

\subsection{Nedd4-2 regulates TrkA intracellular trafficking by modulating its ubiquitination level in early endosomes}

Ubiquitination and ESCRT are well-known factors involved in control of the intracellular trafficking of internalized receptors. The level of ubiquitination and/or ubiquitination type of internalized EGFR have been reported to be necessary for its proper endosomal sorting and degradation (Huang et al. 2006). ESCRT is the factor responsible for sorting the internalized cargoes by recognizing the ubiquitin attached to them and thus preventing their recycling and retrograde trafficking (Raiborg et al. 2009). Therefore, the level and forms of ubiquitination of a receptor determine its fate. The fact that Nedd4-2 is an E3 ubiquitin ligase for TrkA led us to speculate that the depletion of Nedd4-2 might affect the intracellular trafficking of TrkA through modulation of its ubiquitination. To determine whether Nedd4-2 depletion might lead to less TrkA ubiquitination, we compared the ubiquitination level of TrkA in Nedd4-2-depleted DRG neurons with that observed in control neurons upon NGF treatment. We did not find any decrease in TrkA ubiquitination, as would have been expected (Figure R10). In contrast, an increased degree of TrkA ubiquitination was observed in the Nedd4-2-depleted neurons, possibly due to the enhanced TrkA levels and activation. It has been reported that co-transfection of TrkA together with Nedd4-2 in HEK293 cells leads to TrkA ubiquitination (Arevalo et al. 2006b). 
A possible explanation as to why Nedd4-2 depletion does not reduce the ubiquitination levels of TrkA in DRG neurons is that Nedd4-2 might not be the only E3 ubiquitin ligase for TrkA. If Nedd4-2 were indeed the only E3 ubiquitin ligase for TrkA, the depletion of Nedd4-2 would bring about a strong defect in TrkA ubiquitination, but this was not the case. E3 ubiquitin ligases are well known to be responsible for substrate recognition in the process of ubiquitination, but one substrate may be ubiquitinated by more than one E3 ubiquitin ligase. Several E3 ubiquitin ligases have been identified for TrkA: TRAF6 (Geetha et al. 2005; Georgieva et al. 2011), Nedd4-2 (Arevalo et al. 2006b; Georgieva et al. 2011) and Cbl (Takahashi et al. 2011). Also, one E3 ubiquitin ligase can be assigned for several substrates. For example, besides TrkA, AKT is another substrate of TRAF6 (Yang et al. 2009); Nedd4-2 was found to be an E3 ubiquitin ligase responsible for ENaC ubiqutination (Staub et al. 1996), and Cbl is well-known to be responsible for the direct ubiquitination of EGFR. The complexity of the ubiquitination indicates that the depletion of Nedd4-2, one of the E3 ubiquitin ligases for TrkA, may only affect the ubiquitination of TrkA partially. In addition, Nedd4-2 belongs to the family of Nedd4 E3 ubiquitin ligase proteins (Ingham et al. 2004; Yang et al. 2010). All members of the family share structural homology, with a $\mathrm{C} 2$ domain in the $\mathrm{N}$-terminus, several WW domains, and a HECT catalytic domain at the C-terminus (Harvey et al. 1999). According to different reports using genetic approaches to knock out their expression, Nedd4 family members exert specific functions (Fang et al. 2002; Yamashita et al. 2005). However, other studies have indicated that there is also functional compensation and redundancy among different Nedd4 proteins (Myat et al. 2002; Wilkin et al. 2004).

In order to determine how and where Nedd4-2 functions on TrkA, we tested the location of Nedd4-2 in DRG neurons and found that Nedd4-2 was located in early endosomes and late endosomes (Figure R11). Considering the delay taken by activated TrkA to arrive at late endosomes, and the accumulation of activated TrkA in late endosomes at later time-points when Nedd4-2 is depleted (Figure R6), Nedd4-2 may be functioning to ubiquitinate TrkA at the early and late endosome stages. Accordingly, we tested TrkA ubiquitination in early endosomes. We found that unlike the results concerning total TrkA 
ubiquitination, the TrkA present in early endosomes was ubiquitinated to a considerably lesser extent in Nedd4-2-depleted rat DRG neurons than in control neurons (Figure R12). Additionally, by immunofluorescence more activated TrkA was found in the early endosomes in these DRG neurons because of the unchanged internalization rate of TrkA in the Nedd4-2-depleted rat DRGs (Figure R4, R5). Thus, Nedd4-2 depletion results in an increased level of activated, but less ubiquinated, TrkA in early endosomes, which could not be recognized by ESCRT as efficiently as the ubiquitinated TrkA in the control DRG neurons. Consequently, more TrkA becomes available for recycling. All these data suggest that early endosomes would be a potential site where Nedd4-2 functions in TrkA trafficking and sorting to the degradative pathway through ubiquitination. Although this may be a mechanism of Nedd4-2 in regulating TrkA intracellular trafficking, further analysis of the forms of TrkA ubiquitination and the ubiquitination level of TrkA in late endosomes in response to NGF should provide more information and evidence for the roles of Nedd4-2 in TrkA trafficking and sorting.

\subsection{Implications of alterations in the proper TrkA trafficking caused by Nedd4-2}

In neurons, TrkA signalling may emanate from the cell surface as well as from intracellular compartments (Grimes et al. 1996; Zhang et al. 2000). There are reports in the literature supporting the notion that activated TrkA exerts its signalling function from early endosomes (Howe et al. 2001; Wu et al. 2001) as well as from late endosomes (Deinhardt et al. 2006; Hisata et al. 2007). The impaired trafficking of activated TrkA protein caused by Nedd4-2 depletion in sensory neurons resulted in the availability of increased TrkA levels at the cell surface and in intracellular compartments, which consequently brought about an enhanced TrkA signalling as assessed by the activation of PLC- $\gamma$, Akt and MAPK. When we tested the biological functions of the increased signalling pathways using a cell survival assay, we found that the DRG neurons from rat E15.5 embryos with Nedd4-2 depletion survived longer than control neurons in response to NGF deprivation 
(Figure R15). These data were further supported by the enhanced survival of DRG neurons from $\mathrm{KI}$ mice with limited amounts of NGF in vitro as compared with the survival of the DRG neurons of WT mice (Figure R26). Furthermore, we observed more DRG neurons in vivo in the L3 ganglion(ganglia) of KI mice at P0 (Figure R27), possibly due to their increased cell survival during development. In sum, Nedd4-2 affects cell survival in vitro and in vivo by regulating TrkA trafficking and signalling in DRG neurons.

It has been reported that in DRGs TrkA is mainly expressed in nociceptive neurons (Fang et al. 2005). The importance of NGF-TrkA signalling in nociception has been demonstrated in: 1) TrkA and NGF knock-out mice, which fail to respond to painful stimuli (Crowley et al. 1994; Smeyne et al. 1994); 2) patients with CIPA (Indo et al. 1996); 3) the application of antagonists of NGF-TrkA signalling (Mantyh et al. 2011) and anti-TrkA and anti-NGF monoclonal antibodies for pain relief (Ugolini et al. 2007; Abdiche et al. 2008). Thus, as well as testing the biological functions of TrkA ubiquitination mediated by Nedd4-2 in its signalling, trafficking and cell survival in vitro and in vivo, we also tested its roles in nociception in vivo. By using tail-flick, cold plate and paw pressure tests, we applied different kinds of acute stimuli to the mice and found that $\mathrm{KI}$ mice were more sensitive to noxious heat and cold but not to pressure stimuli (Figure R28, R29). Why do $\mathrm{KI}$ mice show a modified response to specific stimuli? There are several lines of evidence that could be explored to address this question: first, TrkA-positive sensory neurons are $C$ fibre and $A \delta$ fibre neurons, which are polymodal and responsible for almost all kinds of pain transduction. However, not all C fibre and Aঠ fibre neurons are TrkA-positive neurons (Table Intr1), suggesting that the processing of thermal and mechanical stimuli may be separate in distinct afferent circuits (Basbaum et al. 2009); second, the majority of TrkA-positive neurons express TRPV1, which has been shown to mediate responses to thermal but not mechanical pain (Mishra et al. 2010); third, ablation of non-peptidergic Mrgprd neurons in DRGs, which do not express TrkA, attenuates mechanosensation, but not thermosensation (Cavanaugh et al. 2009). Therefore, increased signalling and recycling of TrkA in sensory neurons in $\mathrm{KI}$ mice may only result in an alteration to the response to thermal stimuli. By applying in situ hybridization, we tested the expression of 
the nociceptive peptide SP in DRGs at P0 in both WT and KI mice. On one hand, NGF has been reported to regulate SP in adult sensory neurons through both TrkA and p75 receptors (Lindsay et al. 1989; Skoff et al. 2006), and on the other hand there is evidence that mice overexpressing SP display hyperalgesia, as measured by faster reaction times on the tail withdrawal (McLeod et al. 1999). We observed an enhanced expression of SP in KI DRGs (Figure R32), which accounts for the increased sensitivity to acute noxious thermal stimuli in $\mathrm{KI}$ mice. It has been reported that the expression of TRPV1, a well studied and accepted channel for heat sensation, can be enhanced by increased TrkA signalling ( $\mathrm{Ji}$ et al. 2002), and neurons expressing TRPM8, a channel related to cold sensation, also express TrkA (Nealen et al. 2003; Kobayashi et al. 2005; Caspani et al. 2009; Shailendra Kapoor 2012). All these reports suggest that further work addressing the expression of TRPV1 and TRPM8 in DRGs should provide more evidence on how increased TrkA signalling leads to the increased sensitivity of $\mathrm{KI}$ mice to acute noxious thermal stimuli.

To test whether the $\mathrm{KI}$ mice responded to inflammation differently from the WT mice, formalin was injected into the hind paw of the mice and the time each mouse spent licking/biting the injected paw was recorded as an indicator of pain sensation. Formalin injection causes a biphasic profile of the response (Figure R30A): phase I and phase II (Hunskaar et al. 1987). The difference between phase I and phase II lies in that phase I is due to a direct effect on nociceptors, while in phase II a prolonged inflammatory response is involved. We observed a significant increase in the time spent by the $\mathrm{K} 1$ mice biting/licking in both phase I and phase II (Figure R30B), indicating enhanced nociception. The increased sensitivity to formalin of the $\mathrm{KI}$ mice was further confirmed by the increased c-fos expression in the spinal cord two hours after formalin injection (Figure R31), which is a widely recognized marker of neuronal activity (Greenberg et al. 1986; VanElzakker et al. 2008). Formalin injection causes the release of inflammatory mediators including NGF, which is well-known to modulate hypersensitivity in inflamed tissue through binding to TrkA and p75NTR (Nicol et al. 2007). The enhanced NGF-TrkA signalling in the DRGs of KI mice (Figure R25) may lead to hypersensitivity in several ways. First, as reported by us, 
an increased retrograde NGF-TrkA signalling was observed in the DRGs depleted with Nedd4-2 (Figure R14); second, enhanced signalling may regulate the expression of genes related to nociception, such as the neuropeptides SP and CGRP, which are well known to induce hypersensitivity (Oku et al. 1987; Nakamura-Craig et al. 1989; De Felipe et al. 1998); third, according to published data NGF-TrkA signalling can also regulate the activity (Vellani et al. 2001) and surface insertion of TRPV1 (Stein et al. 2006), the channel involved in NGF-mediated hypersensitivity (Caterina et al. 1997; Michael et al. 1999; Ji et al. 2002).

In sum, the results reported here support the notion that correct modulation of the trafficking of TrkA by ubiquitination in response to NGF is a critical event for its functions both in vitro and in vivo. Increased TrkA signalling caused by the inhibition of Nedd4-2 binding in $\mathrm{KI}$ mice renders them more sensitive to acute noxious thermal pain and chronic inflammatory pain. In addition, it is believed that NGF and TrkA may also play important roles in the aetiology of neurodegenerative diseases, such as Alzheimer's. A hypothesis of impaired NGF transport and the subsequent degeneration of cholinergic neurons could be evoked to account for one of the earliest events in Alzheimer's disease (Connor et al. 1996). Future experiments involving the modulation of TrkA protein levels and NGF-mediated signalling may help to further understand the role of NGF in nociception and neurodegenerative diseases. The identification of Nedd4-2 as a molecular determinant of TrkA trafficking represents a potential target for controlling the level of TrkA activation and NGF-TrkA-mediated functions. 
6 Conclusions 
1. Nedd4-2-mediated ubiquitination of TrkA regulates its degradation upon NGF treatment in DRG sensory neurons.

2. Nedd4-2-mediated ubiquitination of TrkA at early endosomes modulates TrkA intracellular trafficking upon NGF treatment.

3. TrkA ubiquitination mediated by Nedd4-2 plays a critical role in NGF-mediated signalling and survival in vitro and in vivo.

4. TrkA ubiquitination mediated by Nedd4-2 modulates nociceptive response to acute noxious thermal and inflammatory stimuli in a TrkA-mutant mouse model, in which Nedd4-2 does not ubiquitinate TrkA. 
7 Resumen en Español 


\section{INTRODUCCIÓN}

\section{Las neurotrofinas y sus receptores}

El funcionamiento correcto del sistema nervioso necesita de distintos factores de crecimiento procedentes de los tejidos inervados para la formación de las conexiones neuronales apropiadas durante el desarrollo y su mantenimiento en el sistema nervioso adulto. Entre otros factores, las neurotrofinas tienen un papel primordial tanto para el desarrollo como para el correcto funcionamiento del sistema nervioso. Las neurotrofinas se han implicado directamente en patologías humanas que incluyen desórdenes neuropsiquiátricos (depresión, esquizofrenia y alteraciones del comportamiento) y enfermedades neurodegenerativas (enfermedad de Alzheimer, Parkinson y Huntington). En mamíferos se han descrito cuatro neurotrofinas, NGF (nerve growth factor), BDNF

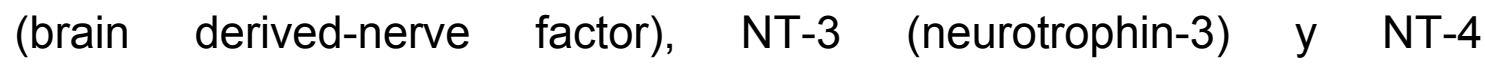
(neurotrophin-4). Todas las neurotrofinas se unen al receptor p75 con una afinidad parecida, mientras que se unen a los receptores tirosina quinasa Trk de forma específica. Así, NGF, BDNF o NT-4 y NT-3 se unen específicamente a TrkA, TrkB o TrkC, repectivamente (Chao 2003; 
Huang et al. 2003). TrkA fue el primer miembro identificado de la familia de receptores Trk (Martin-Zanca et al. 1986) y es el receptor para NGF (Kaplan et al. 1991a; Klein et al. 1991). La expresión de TrkA está principalmente localizada en ganglios del sistema nervioso periférico, aunque también se expresa de forma muy restringida en el sistema nervioso central (Martin-Zanca et al. 1990). Los ratones KO para TrkA y NGF presentan una reducción severa en el número de neuronas de los ganglios raquídeos, cervical superior y trigémino, que se traduce en una insensibilidad a altas temperaturas (Crowley et al. 1994; Smeyne et al. 1994). En humanos, mutaciones inactivantes en el gen TrkA se han asociado directamente con una neuropatía sensorial denominada CIPA (Congenital Insensitivity to Pain with Anhidrosis) (Indo et al. 1996; Indo 2001).

\section{Vías de señalización activadas por TrkA y NGF}

La unión de NGF al receptor TrkA provoca su dimerización y la fosforilación de distintos residuos de tirosina entre los que se encuentran Y490 y Y785. Una vez fosforilados se convierten en lugares de anclaje 
para distintas proteínas que contienen dominios del tipo SH2. Así, en el residuo fosforilado Y490 se une la proteína adaptadora Shc, que a su vez une Grb2 para activar las rutas SOS-Ras-Raf-Mek-Erk y Gab1/2-PI3K-Akt. Por su parte el residuo fosforilado Y785 une PLC $\gamma$ para activar la ruta PKC-Raf-Mek-Erk. NGF activa la ruta de Erk o MAPK de forma transitoria a través de Ras o de forma sostenida a través de Rap1 (York et al. 1998). La activación de Rap1 puede estar mediada mediante la activación de FRS2, que es una proteína adaptadora que se uniría a Y490 fosforilada, (Meakin et al. 1999), o mediante la proteína adaptadora ARMS/Kidins220 (Arevalo et al. 2004; Arevalo et al. 2006a). Las activaciones de estas rutas de señalización determinarían la modulación de otras proteínas citoplásmicas y de factores de transcripción que regularían la expresión de distintos genes.

\section{Ubiquitinación de TrkA}

Los receptores Trk se ubiquitinan una vez que se activan por la unión de su ligando en la parte extracelular. La ubiquitinación es un proceso en el que un pequeño polipéptido de 76 aminoácidos 
denominado ubiquitina se une de forma covalente a un sustrato a través de una lisina. Esta reacción está mediada por la acción sucesiva de tres grupos de enzimas, una E1, que activa la ubiquitina, una E2 que conjuga la ubiquitina y una E3 que bien hace de puente entre la E2 con la ubiquitina conjugada y el sustrato para que sea ubiquitinado (E3 ubiquitina ligasas de la familia RING) o bien une la ubiquitina para transferirla directamente al sustrato (E3 ubiquitina ligasas de la familia HECT). Dependiendo del número de ubiquitinas que se unan al sustrato y la forma de unión tenemos distintos tipos de ubiquitinación: 1) monoubiquitinación, en la que una única ubiquitina se une al sustrato; 2) multi-monoubiquitinación, en la que varias ubiquitinas se unen cada una directamente al sustrato; y 3) poliubiquitinación, en la que un sustrato puede llevar numerosas ubiquitinas que están unidas entre sí. Dependiendo del residuo de lisina de la ubiquitina al que esté unida otra ubiquitina para formar la cadena tenemos distintos tipos de poliubiquitinación (K6, K11, K27, K29, K48 y K63). Cada tipo de ubiquitinación están implicada en distintos procesos celulares. La 
ubiquitinación de receptores tirosina quinasa se ha implicado en la internalización, transporte y degradación de los receptores.

Se ha descrito que los receptores Trk se ubiquitinan en respuesta a su activación (Geetha et al. 2005; Makkerh et al. 2005; Arevalo et al. 2006b). Geetha et al., publicaron que TRAF6 es una E3 ubiquitina ligasa que puede ubiquitinar TrkA mediante poliubiquitinación en K63. Este proceso se produciría mediante la formación de un complejo de TRAF6 con p62 y p75NTR. En 2006, Arevalo et al. identificaron una proteína E3 ubiquitina ligasa, Nedd4-2, que se une, ubiquitina y degrada especificamente el receptor TrkA. Nedd4-2 se une a un motivo del receptor TrkA, PPSY, que está muy conservado en todos los receptores TrkA de distintas especies pero no en los receptores TrkB o TrkC (Arevalo et al. 2006b). Nedd4-2 pertenece a la familia Nedd4 de E3 ubiquitina ligasas. Se ha descrito que puede haber redundancia en las funciones desempeñadas por distintos miembros de la familia (Myat et al. 2002; Wilkin et al. 2004; Yamashita et al. 2005). La expresión exógena de Nedd4-2 en neuronas que dependen de NGF para sobrevivir tiene como resultado la apoptosis de estas células debido a una reducción en los 
niveles de TrkA y consiguientemente de las vías de señalización mediadas por NGF (Arevalo et al. 2006b). Sin embargo, neuronas que dependen de BDNF no están afectadas. Además, la implicación de Nedd4-2 en la ubiquitinación de TrkA ha sido confirmada en estudios realizados por otros grupos independientes (Persaud et al. 2009; Georgieva et al. 2011). En 2011, Takahashi et al. sugirieron que c-Cbl podría ubiquitinar TrkA (Takahashi et al. 2011). A pesar de estos estudios el papel de la ubiquitinación de TrkA en las distintas funciones mediadas por NGF está aún por determinar.

\section{Tráfico de los receptores Trk}

Una vez activados por su ligando, los receptores Trk se internalizan rápidamente para seguir tres posibles rutas: reciclaje a la membrana plasmática, degradación vía lisosomas o, a diferencia de otros receptores tirosina quinasa, transporte retrógrado desde el axón al cuerpo celular. Los receptores TrkA una vez internalizados llegan a los endosomas tempranos donde son clasificados. Por una parte son enviados a los endosomas tardíos y cuerpos multivesiculares para su posterior 
degradación y por otra pueden ser enviados a los endosomas de reciclaje para regresar a la membrana plasmática y comenzar un nuevo ciclo de unión de NGF, activación e internalización. A diferencia de TrkA, los receptores TrkB una vez internalizados son enviados principalmente hacia la ruta de degradación a los lisosomas (Sommerfeld et al. 2000; Chen et al. 2005). Los mecanismos moleculares por los que distintos receptores Trk tienen distinto destino apenas se conocen. 


\section{OBJETIVOS}

Los objetivos que se plantearon para la realización de esta tesis fueron los siguientes:

Objetivo 1- Estudiar el papel de la ubiquitinación de TrkA mediada por Nedd4-2 en la modulación de los niveles y en el tráfico intracelular del receptor en respuesta a NGF en neuronas sensoriales de ganglios raquídeos.

Objetivo 2- Investigar el papel de la ubiquitinatión de TrkA mediada por Nedd4-2 en las funciones de TrkA tanto in vitro como in vivo. 


\section{METODOLOGIA Y DISEÑO EXPERIMENTAL}

Para estudiar los objetivos descritos previamente se utilizaron técnicas en las que el laboratorio del Dr. Juan Carlos Arévalo tiene una experiencia contrastada.

Para la realización de esta tesis se han utilizado las siguientes técnicas:

1- Biología Molecular: Se han usado los métodos básicos de clonación de ADN de acuerdo con los protocolos descritos. Se han generado lentivirus para reducir la expresión de Nedd4-2. Así, se ha utilizado la secuencia 5'-aagtggacaatttaggccgaa-3' correspondiente a los nucleótidos 727-747 del cDNA humano de Nedd4-2 para generar un shRNA que fue posteriormente clonado en el vector lentiviral pLVTHM. La secuencia utilizada tiene una homología del $100 \%$ con la secuencia de rata y ratón. Como control se generó un shRNA correspondiente a la secuencia de 5'-gcgcgctttgtaggattcg-3' del DNA de cloroplastos de Euglena gracilis (Kuratomi et al. 2005). Los lentivirus se generaron mediante la co-transfección en células HEK293FT de los plásmidos pLVTHM generados junto con otros dos plásmidos (pMD2.G y pPAX2) 
para generar lentivirus deficientes en replicación. Los sobrenadantes de los cultivos que contienen los lentivirus fueron utilizados para infectar neuronas sensoriales.

2- Cultivo de líneas celulares y de neuronas primarias: Se han utilizado cultivos celulares de las líneas celulares HEK293, HEK293FT y PC12. Las neuronas primarias se obtuvieron de los ganglios raquídeos de embriones E15.5 de rata y E13.5 de ratón. Se sembraron en placas tratadas con Matrigel en un medio que contiene un $10 \%$ de suero bovino fetal y NGF (50 ng/ml) o BDNF (25 ng/ml). Al siguiente día, el medio se sustituyó por medio neurobasal suplementado con B27 y glutamina junto con un antimitótico (5-fluorouridina) y se le añadió la correspondiente neurotrofina. Las células gliales y fibroblastos contaminantes del cultivo desaparecieron 2-3 días después.

3- Bioquímica: Las proteínas se analizaron mediante la técnica de western blot. Las proteínas de la superficie celular se marcaron con biotina a $4^{\circ} \mathrm{C}$ y se separaron del resto usando una matriz con avidina, que 
une específicamente biotina, y posteriormente, se identificaron mediante análisis de western blot. Se realizaron distintas aproximaciones con la biotina para estudiar el marcaje de superficie, la degradación y la internalizacion. La ubiquitinación del receptor TrkA se analizó mediante una inmunoprecipitación del receptor para posteriormente realizar western blot utilizando anticuerpos que reconocen ubiquitina.

4- Biología celular: Para los análisis de inmunofluorescencia se utilizaron cultivos de neuronas sensoriales en cubreobjetos tratados con poly-lysina y matrigel. Las neuronas se infectaron o transfectaron para regular la expresión de distintas proteínas y posteriormente, se estimularon como se indica en cada experimento. Los cubres con las células se recogieron y procesaron para realizar las inmunofluorescencias con los correspondientes anticuerpos.

5- Animales transgénicos: Se generó un ratón transgénico en colaboración con el Dr. Lino Tessarollo ( $\mathrm{NCl})$ en el que se introdujo una mutación en el codón correspondiente a la prolina 782 que se cambió a 
serina (TrkAP782S). El ratón TrkAP782S generado se utilizó para obtener neuronas de ganglios raquídeos que se usaron para estudios de tráfico del receptor, señalización y supervivencia. Además estos ratones se utilizaron para estudios anatómicos y de comportamiento.

6- Estudios de comportamiento: El ratón TrkAP782S se utilizó para distintos estudios de tipo comportamental en los que NGF/TrkA se han implicado directamente. Así, se realizaron estudios de desviación de la cola en respuesta a calor (tail flick), de sensibilidad al frío (cold plate), de sensibilidad a dolor por presión (Randall-Sellito test) y de sensibilidad a dolor inflamatorio mediante la inyección de formalina en la pata trasera.

El mantenimiento de la colonia de ratones y los estudios llevados a cabo con ellos se realizaron de acuerdo con los protocolos aprobados por el Comité de Bioética de la Universidad de Salamanca y siguiendo las directrices marcadas por la Comunidad Europea. 


\section{RESULTADOS}

\section{Nedd4-2 modula el tráfico y los niveles de TrkA regulando}

\section{su degradación.}

Previamente, se había descrito que la sobreexpresión de Nedd4-2 in vitro produce un descenso en los niveles del receptor TrkA (Arevalo et al. 2006b). Sin embargo, se desconocían los efectos de Nedd4-2 expresada endógenamente en neuronas sensoriales. Para la realización de esta tesis doctoral se generaron lentivirus que expresaban un shRNA control y otro que reconocía específicamente la secuencia de Nedd4-2, lo que permitió la reducción de los niveles endógenos de Nedd4-2 en neuronas en cultivo infectadas con lentivirus que expresan el shRNA contra Nedd4-2 (Figure R1). Este descenso de Nedd4-2 provocó un incremento tanto en los niveles totales como en los de superficie del receptor TrkA, (Figure R2). Sin embargo, los niveles del receptor de neurotrofinas p75 no se vieron afectados.

Para estudiar la degradación, reciclaje e internalización del receptor TrkA que se activa en la membrana plasmática en respuesta a NGF se utilizaron una serie de aproximaciones que consistieron en el 
marcaje de las proteínas de membrana con biotina para poder seguirlas.

Los resultados obtenidos indican que la degradación del receptor TrkA activo, pero no de $\mathrm{p} 75$, se produjo de manera más lenta cuando se redujeron los niveles de Nedd4-2 (Figure R3). Estos datos sugirieron que la proteína Nedd4-2 estaría implicada en el proceso de degradación del receptor TrkA. También se realizaron experimentos para estudiar si Nedd4-2 podría afectar a la internalización del receptor TrkA. Los resultados obtenidos indicaron que la reducción de los niveles de Nedd4-2 no altera la internalización de TrkA ni de p75 en respuesta a NGF (Figure R4).

Todos estos datos obtenidos referentes al papel de Nedd4-2 en la degradación e internalización de TrkA se complementaron con experimentos de immunofluorescencia que indicaron que hay una acumulación de receptores TrkA activos en determinados compartimentos celulares cuando se redujo la proteína Nedd4-2. Concretamente, se observó que los receptores TrkA llegan a los endosomas tempranos con independencia de los niveles de Nedd4-2 (Figure R5), pero tardan más tiempo en llegar a los endosomas tardíos 
(Figure R6) cuando se reducen los niveles de Nedd4-2. Estos resultados fueron además confirmados utilizando fraccionamientos subcelulares para obtener fracciones enriquecidas en endosomas tempranos y tardíos. De este modo, se observó que cuando se elimina Nedd4-2 los receptores TrkA se acumulan en endosomas tempranos y tardíos a distintos tiempos que cuando Nedd4-2 está presente (Figure R7). Todos estos datos indican que Nedd4-2 modula el tráfico de los receptores TrkA en respuesta a NGF.

Cuando se estudió cuál puede ser el destino de los receptores TrkA que se acumulan al reducir los niveles de Nedd4-2, se observó que había más receptores TrkA en endosomas de reciclaje (Figure R8) y que el reciclaje de TrkA a la membrana se incrementó una vez que ha sido internalizado en respuesta a NGF (Figure R9). Por lo tanto, si los receptores TrkA no son enviados a la degradación por la falta de Nedd4-2 vuelven más la membrana plasmática.

\section{Nedd4-2 modula la ubiquitinación de TrkA en endosomas}

\section{tempranos}


Posteriormente, se investigó la ubiquitinación del receptor TrkA en respuesta a NGF cuando se redujeron los niveles de Nedd4-2. Sorprendentemente, se observó que no hubo un descenso en los niveles de ubiquitinación del receptor TrkA, al contrario, parecía que había un incremento (Figure R10), que podría ser debido al incremento en los niveles y la activación de TrkA. Para tratar de explicar estos resultados realizamos estudios de inmunofluorescencia para identificar la localización de Nedd4-2. Se detectó que Nedd4-2 estaba presente en endosomas tempranos y tardíos por la co-localización con los marcadores EEA1 y Rab5 (endosomas tempranos) y Rab7 (endosomas tardíos) (Figure R11). A la vista de estos datos se realizaron estudios adicionales de ubiquitinación de los receptores TrkA obtenidos de endosomas tempranos, los cuales indicaron que Nedd4-2 ubiquitina el receptor TrkA en estos compartimentos (Figure R12). Todos estos resultados juntos indican que Nedd4-2 está presente en endosomas tempranos y tardíos, que ubiquitina el receptor TrkA en compartimentos específicos y que esta modificación del receptor es necesaria para que TrkA progrese en la ruta de degradación hacia los lisosomas. 


\section{Nedd4-2 regula la señalización y la supervivencia de}

\section{neuronas dependientes de NGF}

Posteriormente, se realizaron distintos experimentos para estudiar como la reducción de Nedd4-2 afectaba la señalización de NGF mediada por TrkA. Como resultado del aumento de TrkA en neuronas con niveles reducidos de Nedd4-2 se observó un incremento concomitante en las vías de señalización activadas por la adición de NGF a las neuronas. Así, se produjo un incremento en la activación de TrkA, PLC $\gamma$, MAPK y Akt en las neuronas tratadas con NGF que presentan niveles disminuidos de Nedd4-2 (Figure R13). Estos resultados sugieren que Nedd4-2 modula los niveles del receptor activo y las cascadas de señalización en respuesta a NGF.

Las neuronas sensoriales presentan su cuerpo celular en los ganglios raquídeos o en el ganglio trigémino mientras que su axones proyectan bien a la médula espinal o inervan distintos órganos o la piel. La mayor parte de estas neuronas obtienen las señales necesarias para sobrevivir mediadas por la activación del receptor TrkA mediante las 
neurotrofinas a partir de los tejidos que inervan. Por lo tanto, la activación de TrkA debe viajar a través del axón desde la terminal al cuerpo neuronal mediante un transporte retrógrado. Para estudiar si Nedd4-2 modulaba el transporte retrógrado de TrkA, se utilizaron cámaras microfluídicas que permiten la separación del cuerpo neuronal de las terminales axónicas in vitro. Así, se obtuvieron neuronas de ganglios raquídeos, se sembraron en las cámaras microfluídicas y los axones fueron capaces de pasar al compartimento distal. Estas neuronas fueron infectadas con lentivirus para eliminar la expresión de Nedd4-2, posteriormente, se estimularon con NGF en el compartimento distal y finalmente, se recogieron extractos correspondientes al compartimento que tiene los cuerpos neuronales. Los análisis de western blot indicaron que el transporte retrógrado de TrkA activado en las neuronas sensoriales $\sin$ Nedd4-2 se incrementó con respecto al de neuronas control (Figure R14). Por lo tanto, Nedd4-2 modula el transporte retrógrado de TrkA activado.

Además, como las neuronas sensoriales dependen de NGF o BDNF para su supervivencia se realizaron experimentos en los que se 
eliminó la expresión de Nedd4-2, después se retiró el NGF o BDNF y se analizaron el número de neuronas apoptóticas. Las neuronas dependientes de NGF presentaron una supervivencia incrementada cuando se les eliminó Nedd4-2 mientras que las dependientes de BDNF no (Figure R15). Estos resultados indicaron que Nedd4-2 modula la supervivencia de las neuronas dependientes de NGF mediante la regulación de los niveles de TrkA pero no las dependientes de BDNF.

\section{El receptor TrkAP782S, insensible a la acción de Nedd4-2, presenta una degradación y tráfico alterados}

Se ha descrito que Nedd4-2 tiene numerosos sustratos. Para confirmar de modo independiente que los efectos de Nedd4-2 no eran un resultado indirecto sobre TrkA se realizaron otras aproximaciones experimentales. En colaboración con el Dr. Lino Tessarollo (National Cancer Institute, USA) se generó un ratón modificado genéticamente que expresa un receptor TrkA mutante (knock-in) que no une Nedd4-2 y, por tanto, es insensible a la acción de esta E3 ubiquitina ligasa (Figure 
R16). Previamente, se había identificado que el dominio PPXY presente en TrkA, pero no TrkB, era el responsable entre la unión de TrkA y Nedd4-2 (Arevalo et al. 2006b). El codón codificante para la Prolina 782 fue mutado a un codón codificante para Serina, el aminoácido presente en TrkB. De esta manera se destruyó el motivo PPXY de TrkA. La construcción y el "screening" de los clones de las células ES se muestran en la Figure R17. Se obtuvieron neuronas de ganglios raquídeos a partir de embriones E13.5 procedentes de ratones silvestres y knock-in para realizar una serie de experimentos. Lo primero que se hizo fue mirar los niveles de expresión de TrkA. La hipótesis que se barajó, basada en los datos obtenidos con la eliminación de Nedd4-2, era que los niveles de TrkAP782S deberían incrementarse debido a la falta de unión y ubiquitinación por Nedd4-2 de este receptor mutante. Sorprendentemente, se observó un descenso significativo (23\%) en los niveles de TrkA en los ratones mutantes (Figure R18), lo contrario de lo esperado. Para explicar estas discrepancias entre los datos obtenidos con las muestras de rata y ratón se realizaron experimentos en los que se analizaron los niveles de ARNm producidos por el ratón mutante en 
las neuronas de ganglios raquídeos. Se observó un descenso en los niveles de ARNm (40\%) en los ratones knock-in mediante PCR cuantitativa (Figure R19), que podrían ser responsables del descenso en los niveles de proteína. Cuando se analizó la relación entre la cantidad de proteína presente y el ARNm producido, se vio un que había un incremento en el ratón knock-in, lo que indicó que para una misma cantidad de ARNm se producía más TrkAP782S que TrkA silvestre. Estos datos sugirieron que la proteína TrkAP782S podría ser más estable que TrkA.

Para estudiar si TrkAP782S era más estable que TrkA se realizaron experimentos de degradación con neuronas de los ratones mediante biotinilización, de la misma manera que la descrita anteriormente con las neuronas de rata. Se observó que TrkAP782S es más resistente a la degradación en respuesta a NGF que el receptor silvestre (Figure R20). Estos datos se complementaron mediante estudios adicionales del tráfico del receptor en los que se encontró que el receptor mutante se acumulaba en endosomas tempranos (Figure R21), no llegaba de forma eficiente a los endosomas tardíos (Figure R22) y volvía más a la 
membrana en endosomas de reciclaje (Figure R23). Por lo tanto, el receptor TrkAP782S una vez activado era más resistente a la degradación debido a un problema de tráfico entre distintos compartimentos. Posteriormente, se estudió la ubiquitinación del receptor TrkAP782S. Se observó un descenso en la ubiquitinación del receptor TrkAP782S respecto al TrkA silvestre (Figure R24), que no era debida a ningún problema en la activación del receptor ya que presentaba un incremento en su fosforilación (Figure R24). Por lo tanto, el receptor TrkAP782S se degrada menos que el silvestre debido a un defecto en su ubiquitinación.

\section{La expresión de TrkAP782S confiere un incremento en la} señalización y supervivencia de las neuronas que lo expresan Además, se investigó la activación de TrkAP782S y de las vías de señalización en respuesta a NGF. Se observó un incremento en la activación del receptor TrkAP782S usando neuronas en cultivo procedentes de este ratón knock-in que iba acompañado de un incremento en la señalización con respecto a neuronas obtenidas de un 
ratón silvestre (Figure R25). Estos datos fueron muy similares a los previamente descritos cuando se eliminó la expresión de Nedd4-2. Para estudiar las consecuencias funcionales de este aumento en la señalización en TrkAP782S, se realizaron experimentos de supervivencia utilizando neuronas sensoriales, las cuales dependen de la señalización dependiente de NGF. Usando NGF a una concentración de $25 \mathrm{ng} / \mathrm{ml}$ observamos aproximadamente la misma supervivencia en las neuronas que expresaban TrkA silvestre y TrkAP782S. Sin embargo, las neuronas que expresaban TrkAP782S presentaban una mayor supervivencia (105\%) que las que expresaban TrkA (20\%) cuando se cultivaron en concentraciones limitantes de NGF $(1 \mathrm{ng} / \mathrm{ml})$ respecto a las que sobrevivían con $25 \mathrm{ng} / \mathrm{ml}$ de NGF (Figure R26). Estos datos fueron además confirmados in vivo cuando se cuantificó el número de neuronas presentes en los ganglios raquídeos L3. Los ratones knock-in presentaron un incremento significativo en el número de neuronas respecto a los silvestres (Figure R27). Estos datos indicaron que la expresión del receptor TrkAP782S confiere una ventaja para la supervivencia de las neuronas que lo expresan debido al incremento en 
su activación y en las vías de señalización dependientes de NGF al no ser afectado por la ubiquitinación mediada por Nedd4-2.

\section{Los ratones knock-in que expresan TrkAP782S presentan} un incremento en la sensibilidad a dolor térmico e inflamatorio

Previamente se había descrito que los ratones "knock-out" para TrkA y NGF son insensibles a estímulos dolorosos por calor (Crowley et al. 1994; Smeyne et al. 1994). Por lo tanto, se realizaron estudios en ratones silvestres y knock-in para investigar su sensibilidad a dolor por calor, frío y presión en colaboración con la Dra. Raquel Rodríguez (INCyL, Universidad de Salamanca). Los resultados obtenidos indicaron que el ratón knock-in es más sensible que el silvestre a un estímulo doloroso producido por calor o frío (Figure R28). Sin embargo el ratón knock-in se comporta de la misma manera que el silvestre en respuesta a un estímulo mecánico (Figure 29). Estos datos indicaron que la expresión de TrkAP782S confiere un incremento en la sensibilidad de los ratones knock-in a estímulos dolorosos de tipo térmico. 
Posteriormente, se realizaron otros estudios para comprobar la sensibilidad de los ratones mutantes a estímulos de tipo inflamatorio, en los que TrkA/NGF han sido directamente implicados. Así, la inflamación inducida mediante la inyección de formalina en la pata de los ratones, produjo un incremento en el tiempo que los ratones knock-in experimentaron distintos síntomas asociados al dolor inflamatorio en comparación con los silvestres (Figure R30). Este aumento en los tiempos de respuesta en los ratones mutantes se correlacionó con el incremento en la actividad neuronal en la médula espinal medida mediante la expresión de c-fos (Figure R31). Por lo tanto, los ratones knock-in presentaron una sensibilidad a dolor inflamatorio incrementada.

Finalmente, se realizaron hibridaciones in situ para estudiar la expresión de los ARNm correspondientes a los genes Sustancia $P$ y CGRP, dos moléculas que se han relacionado directamente con la sensibilidad al dolor y que son modulados por la activación del receptor TrkA en respuesta a NGF. Los resultados obtenidos indicaron que la expresión de Sustancia $P$ está incrementada en gran medida en los 
ganglios raquídeos procedentes de los ratones knock-in mientras que se observó un ligero aumento en los niveles de CGRP (Figure R32).

Todos los datos obtenidos con el ratón knock-in apoyan los datos anteriores obtenidos con las neuronas sensoriales de rata en las que se redujo la expresión de Nedd4-2 e indican que la ubiquitinación de TrkA mediada por Nedd4-2 juega un papel muy importante en las funciones mediadas por NGF/TrkA. 


\section{CONCLUSIONES}

1. La ubiquitinación de TrkA mediada por Nedd4-2 modula la degradación de TrkA en neuronas sensoriales.

2. La ubiquitinación de TrkA mediada por Nedd4-2 en los endosomas tempranos modula el tráfico intracelular del receptor en respuesta a NGF.

3. La ubiquitinación de TrkA mediada por Nedd4-2 juega un papel crucial en la señalización mediada por NGF y en la supervivencia de neuronas sensoriales in vitro e in vivo.

4. La ubiquitinación de TrkA mediada por Nedd4-2 modula la respuesta nociceptiva a estímulos térmicos e inflamatorios en un modelo de ratón que expresa un receptor TrkA insensible a la acción de Nedd4-2. 
Resumen en Español 


\section{References}


Abdiche, Y. N., D. S. Malashock, et al. (2008). "Probing the binding mechanism and affinity of tanezumab, a recombinant humanized anti-NGF monoclonal antibody, using a repertoire of biosensors." Protein Sci 17(8): 1326-1335.

Abrams, E. J., J. Weedon, et al. (1998). "Association of human immunodeficiency virus (HIV) load early in life with disease progression among HIV-infected infants. New York City Perinatal HIV Transmission Collaborative Study Group." J Infect Dis 178(1): 101-108.

Aftab, D. T., J. Kwan, et al. (1997). "Ras-independent transformation by v-Src." Proc Natl Acad Sci U S A 94(7): 3028-3033.

Aguilar, R. C. and B. Wendland (2005). "Endocytosis of membrane receptors: two pathways are better than one." Proc Natl Acad Sci U S A 102(8): 2679-2680.

Allen, S. J. and D. Dawbarn (2006). "Clinical relevance of the neurotrophins and their receptors." Clin Sci (Lond) 110(2): 175-191.

Amaya, F., G. Shimosato, et al. (2004). "NGF and GDNF differentially regulate TRPV1 expression that contributes to development of inflammatory thermal hyperalgesia." Eur J Neurosci 20(9): 2303-2310.

Angers, A., A. R. Ramjaun, et al. (2004). "The HECT domain ligase itch ubiquitinates endophilin and localizes to the trans-Golgi network and endosomal system." J Biol Chem 279(12): 11471-11479.

Ardley, H. C. and P. A. Robinson (2005). "E3 ubiquitin ligases." Essays Biochem 41: 15-30.

Arevalo, J. C., D. B. Pereira, et al. (2006a). "Identification of a switch in neurotrophin signaling by selective tyrosine phosphorylation." J Biol Chem 281(2): 1001-1007.

Arevalo, J. C., J. Waite, et al. (2006b). "Cell survival through Trk neurotrophin receptors is differentially regulated by ubiquitination." Neuron 50(4): 549-559.

Arevalo, J. C. and S. H. Wu (2006c). "Neurotrophin signaling: many exciting surprises!" Cell Mol Life Sci 63(13): 1523-1537.

Arevalo, J. C., H. Yano, et al. (2004). "A unique pathway for sustained neurotrophin signaling through an ankyrin-rich membrane-spanning protein." EMBO J 23(12): 2358-2368.

Armstrong, J. (2000). "How do Rab proteins function in membrane traffic?" Int J Biochem Cell Biol 32(3): 303-307.

Averill, S., S. B. McMahon, et al. (1995). "Immunocytochemical localization of trkA receptors in chemically identified subgroups of adult rat sensory neurons." Eur J Neurosci 7(7): 1484-1494.

Basbaum, A. I., D. M. Bautista, et al. (2009). "Cellular and molecular mechanisms of pain." 139(2): 267-284.

Bedford, L., J. Lowe, et al. (2011). "Ubiquitin-like protein conjugation and the ubiquitin-proteasome system as drug targets." Nat Rev Drug Discov 10(1): 29-46.

Benemei, S., P. Nicoletti, et al. (2009). "CGRP receptors in the control of pain and inflammation." Curr Opin Pharmacol 9(1): 9-14.

Berridge, M. J. and R. F. Irvine (1984). "Inositol trisphosphate, a novel second messenger in cellular signal transduction." Nature 312(5992): 315-321.

Bibel, M., E. Hoppe, et al. (1999). "Biochemical and functional interactions between the neurotrophin receptors trk and p75NTR." EMBO J 18(3): 616-622.

Bilderback, T. R., V. R. Gazula, et al. (1999). "Caveolin interacts with Trk A and p75(NTR) and regulates neurotrophin signaling pathways." J Biol Chem 274(1): 257-263. 
Blake, T. J., M. Shapiro, et al. (1991). "The sequences of the human and mouse c-cbl proto-oncogenes show v-cbl was generated by a large truncation encompassing a proline-rich domain and a leucine zipper-like motif." Oncogene 6(4): 653-657.

Blume-Jensen, P. and T. Hunter (2001). "Oncogenic kinase signalling." Nature 411(6835): 355-365.

Boase, N. A., G. Y. Rychkov, et al. (2011). "Respiratory distress and perinatal lethality in Nedd4-2-deficient mice." Nat Commun 2: 287.

Brunet, A., A. Bonni, et al. (1999). "Akt promotes cell survival by phosphorylating and inhibiting a Forkhead transcription factor." Cell 96(6): 857-868.

Casademunt, E., B. D. Carter, et al. (1999). "The zinc finger protein NRIF interacts with the neurotrophin receptor p75(NTR) and participates in programmed cell death." EMBO J 18(21): 6050-6061.

Caspani, O., S. Zurborg, et al. (2009). "The contribution of TRPM8 and TRPA1 channels to cold allodynia and neuropathic pain." PLoS One 4(10): e7383.

Caterina, M. J., M. A. Schumacher, et al. (1997). "The capsaicin receptor: a heat-activated ion channel in the pain pathway." Nature 389(6653): 816-824.

Cattaneo, A. (2010). "Tanezumab, a recombinant humanized mAb against nerve growth factor for the treatment of acute and chronic pain." Curr Opin Mol Ther 12(1): 94-106.

Cavanaugh, D. J., H. Lee, et al. (2009). "Distinct subsets of unmyelinated primary sensory fibers mediate behavioral responses to noxious thermal and mechanical stimuli." Proc Natl Acad Sci U S A 106(22): 9075-9080.

Chang, M. S., J. C. Arevalo, et al. (2004). "Ternary complex with Trk, p75, and an ankyrin-rich membrane spanning protein." J Neurosci Res 78(2): 186-192.

Chao, M. V. (2003). "Neurotrophins and their receptors: a convergence point for many signalling pathways." Nat Rev Neurosci 4(4): 299-309.

Chao, M. V., M. A. Bothwell, et al. (1986). "Gene transfer and molecular cloning of the human NGF receptor." Science 232(4749): 518-521.

Chen, C. and L. E. Matesic (2007). "The Nedd4-like family of E3 ubiquitin ligases and cancer." Cancer Metastasis Rev 26(3-4): 587-604.

Chen, Z. Y., A. leraci, et al. (2005). "A novel endocytic recycling signal distinguishes biological responses of Trk neurotrophin receptors." Mol Biol Cell 16(12): 5761-5772.

Chittka, A. and M. V. Chao (1999). "Identification of a zinc finger protein whose subcellular distribution is regulated by serum and nerve growth factor." Proc Natl Acad Sci U S A 96(19): 10705-10710.

Choi, D. Y., J. J. Toledo-Aral, et al. (2001). "Sustained signaling by phospholipase C-gamma mediates nerve growth factor-triggered gene expression." Mol Cell Biol 21(8): 2695-2705.

Claude, P., E. Hawrot, et al. (1982). "Binding, internalization, and retrograde transport of 125I-nerve growth factor in cultured rat sympathetic neurons." J Neurosci 2(4): 431-442.

Connor, B., D. Young, et al. (1996). "Trk receptor alterations in Alzheimer's disease." Brain Res Mol Brain Res 42(1): 1-17. 
Crowley, C., S. D. Spencer, et al. (1994). "Mice lacking nerve growth factor display perinatal loss of sensory and sympathetic neurons yet develop basal forebrain cholinergic neurons." Cell 76(6): 1001-1011.

Dalton, S. and R. Treisman (1992). "Characterization of SAP-1, a protein recruited by serum response factor to the c-fos serum response element." Cell 68(3): 597-612.

Davies, A. M. (1986). "The survival and growth of embryonic proprioceptive neurons is promoted by a factor present in skeletal muscle." Dev Biol 115(1): 56-67.

Davies, A. M. (2000). "Neurotrophins: neurotrophic modulation of neurite growth." Curr Biol 10(5): R198-200.

de Carlos, F., J. Cobo, et al. (2006). "Abnormal development of pacinian corpuscles in double trkB;trkC knockout mice." Neurosci Lett 410(3): 157-161.

De Felipe, C., J. F. Herrero, et al. (1998). "Altered nociception, analgesia and aggression in mice lacking the receptor for substance P." Nature 392(6674): 394-397.

Deinhardt, K., S. Salinas, et al. (2006). "Rab5 and Rab7 control endocytic sorting along the axonal retrograde transport pathway." Neuron 52(2): 293-305.

Delcroix, J. D., J. S. Valletta, et al. (2003). "NGF signaling in sensory neurons: evidence that early endosomes carry NGF retrograde signals." Neuron 39(1): 69-84.

Di Marco, E., M. Mathor, et al. (1993). "Nerve growth factor binds to normal human keratinocytes through high and low affinity receptors and stimulates their growth by a novel autocrine loop." J Biol Chem 268(30): 22838-22846.

Djouhri, L. and S. N. Lawson (2004). "Abeta-fiber nociceptive primary afferent neurons: a review of incidence and properties in relation to other afferent A-fiber neurons in mammals." Brain Res Brain Res Rev 46(2): 131-145.

Duman, R. S. (2004). "Role of neurotrophic factors in the etiology and treatment of mood disorders." Neuromolecular Med 5(1): 11-25.

Fahnestock, M. (1991). "Structure and biosynthesis of nerve growth factor." Curr Top Microbiol Immunol 165: 1-26.

Fang, D., C. Elly, et al. (2002). "Dysregulation of T lymphocyte function in itchy mice: a role for Itch in TH2 differentiation." Nat Immunol 3(3): 281-287.

Fang, X., L. Djouhri, et al. (2005). "trkA is expressed in nociceptive neurons and influences electrophysiological properties via Nav1.8 expression in rapidly conducting nociceptors." J Neurosci 25(19): 4868-4878.

Galan, J. M. and R. Haguenauer-Tsapis (1997). "Ubiquitin lys63 is involved in ubiquitination of a yeast plasma membrane protein." EMBO J 16(19): 5847-5854.

Garber, K. (2011). "Fate of novel painkiller mAbs hangs in balance." Nat Biotechnol 29(3): 173-174.

Geetha, T., J. Jiang, et al. (2005). "Lysine 63 polyubiquitination of the nerve growth factor receptor TrkA directs internalization and signaling." Mol Cell 20(2): 301-312.

Georgieva, M. V., Y. de Pablo, et al. (2011). "Ubiquitination of TrkA by Nedd4-2 regulates receptor lysosomal targeting and mediates receptor signaling." J Neurochem 117(3): 479-493.

Ghilardi, J. R., K. T. Freeman, et al. (2011). "Sustained blockade of neurotrophin receptors TrkA, TrkB and TrkC reduces non-malignant skeletal pain but not the maintenance of sensory and sympathetic nerve fibers." Bone 48(2): 389-398. 
Grabbe, C., K. Husnjak, et al. (2011). "The spatial and temporal organization of ubiquitin networks." Nat Rev Mol Cell Biol 12(5): 295-307.

Grant, B. D. and J. G. Donaldson (2009). "Pathways and mechanisms of endocytic recycling." Nat Rev Mol Cell Biol 10(9): 597-608.

Greenberg, M. E., A. L. Hermanowski, et al. (1986). "Effect of protein synthesis inhibitors on growth factor activation of c-fos, c-myc, and actin gene transcription." Mol Cell Biol 6(4): 1050-1057.

Greenberg, M. E., Z. Siegfried, et al. (1987). "Mutation of the c-fos gene dyad symmetry element inhibits serum inducibility of transcription in vivo and the nuclear regulatory factor binding in vitro." Mol Cell Biol 7(3): 1217-1225.

Grimes, M. L., J. Zhou, et al. (1996). "Endocytosis of activated TrkA: evidence that nerve growth factor induces formation of signaling endosomes." $\mathrm{J}$ Neurosci 16(24): 7950-7964.

Grovdal, L. M., E. Stang, et al. (2004). "Direct interaction of Cbl with pTyr 1045 of the EGF receptor (EGFR) is required to sort the EGFR to lysosomes for degradation." Exp Cell Res 300(2): 388-395.

Halvorson, K. G., K. Kubota, et al. (2005). "A blocking antibody to nerve growth factor attenuates skeletal pain induced by prostate tumor cells growing in bone." $\underline{\text { Cancer Res }}$ 65(20): 9426-9435.

Harmar, A., J. G. Schofield, et al. (1980). "Cycloheximide-sensitive synthesis of substance P by isolated dorsal root ganglia." Nature 284(5753): 267-269.

Harrington, A. W., B. Leiner, et al. (2004). "Secreted proNGF is a pathophysiological death-inducing ligand after adult CNS injury." Proc Natl Acad Sci U S A 101(16): 6226-6230.

Harrington, A. W., C. St Hillaire, et al. (2011). "Recruitment of actin modifiers to TrkA endosomes governs retrograde NGF signaling and survival." Cell 146(3): 421-434.

Harvey, K. F., A. Dinudom, et al. (1999). "All three WW domains of murine Nedd4 are involved in the regulation of epithelial sodium channels by intracellular $\mathrm{Na}+. " \mathrm{~J}$ Biol Chem 274(18): 12525-12530.

Hasegawa, Y., S. Yamagishi, et al. (2004). "p75 neurotrophin receptor signaling in the nervous system." Biotechnol Annu Rev 10: 123-149.

Hempstead, B. L., R. B. Birge, et al. (1994). "Expression of the v-crk oncogene product in PC12 cells results in rapid differentiation by both nerve growth factor- and epidermal growth factor-dependent pathways." Mol Cell Biol 14(3): 1964-1971.

Hempstead, B. L., D. Martin-Zanca, et al. (1991). "High-affinity NGF binding requires coexpression of the trk proto-oncogene and the low-affinity NGF receptor." Nature 350(6320): 678-683.

Hershko, A. and A. Ciechanover (1998). "The ubiquitin system." Annu Rev Biochem 67: 425-479.

Hershko, A., A. Ciechanover, et al. (2000). "Basic Medical Research Award. The ubiquitin system." Nat Med 6(10): 1073-1081.

Herzberg, U., E. Eliav, et al. (1997). "NGF involvement in pain induced by chronic constriction injury of the rat sciatic nerve." Neuroreport 8(7): 1613-1618. 
Hisata, S., T. Sakisaka, et al. (2007). "Rap1-PDZ-GEF1 interacts with a neurotrophin receptor at late endosomes, leading to sustained activation of Rap1 and ERK and neurite outgrowth." J Cell Biol 178(5): 843-860.

Howe, C. L., J. S. Valletta, et al. (2001). "NGF signaling from clathrin-coated vesicles: evidence that signaling endosomes serve as a platform for the Ras-MAPK pathway." Neuron 32(5): 801-814.

Hua, X. Y., P. Chen, et al. (1999). "Intrathecal substance P-induced thermal hyperalgesia and spinal release of prostaglandin E2 and amino acids." Neuroscience 89(2): 525-534.

Huang, E. J. and L. F. Reichardt (2001). "Neurotrophins: roles in neuronal development and function." Annu Rev Neurosci 24: 677-736.

Huang, E. J. and L. F. Reichardt (2003). "Trk receptors: roles in neuronal signal transduction." Annu Rev Biochem 72: 609-642.

Huang, F., D. Kirkpatrick, et al. (2006). "Differential regulation of EGF receptor internalization and degradation by multiubiquitination within the kinase domain." Mol Cell 21(6): 737-748.

Hummler, E. (1999). "Implication of ENaC in salt-sensitive hypertension." J Steroid Biochem Mol Biol 69(1-6): 385-390.

Hunskaar, S. and K. Hole (1987). "The formalin test in mice: dissociation between inflammatory and non-inflammatory pain." Pain 30(1): 103-114.

Ikeda, F. and I. Dikic (2008). "Atypical ubiquitin chains: new molecular signals. 'Protein Modifications: Beyond the Usual Suspects' review series." EMBO Rep 9(6): 536-542.

Indo, Y. (2001). "Molecular basis of congenital insensitivity to pain with anhidrosis (CIPA): mutations and polymorphisms in TRKA (NTRK1) gene encoding the receptor tyrosine kinase for nerve growth factor." Hum Mutat 18(6): 462-471.

Indo, Y. (2010). "Nerve growth factor, pain, itch and inflammation: lessons from congenital insensitivity to pain with anhidrosis." Expert Rev Neurother 10(11): 1707-1724.

Indo, Y., M. Tsuruta, et al. (1996). "Mutations in the TRKA/NGF receptor gene in patients with congenital insensitivity to pain with anhidrosis." Nat Genet 13(4): 485-488.

Ingham, R. J., G. Gish, et al. (2004). "The Nedd4 family of E3 ubiquitin ligases: functional diversity within a common modular architecture." Oncogene 23(11): 1972-1984.

Ishida, T., S. Mizushima, et al. (1996). "Identification of TRAF6, a novel tumor necrosis factor receptor-associated factor protein that mediates signaling from an amino-terminal domain of the CD40 cytoplasmic region." J Biol Chem 271(46): 28745-28748.

Ji, R. R., T. A. Samad, et al. (2002). "p38 MAPK activation by NGF in primary sensory neurons after inflammation increases TRPV1 levels and maintains heat hyperalgesia." Neuron 36(1): 57-68.

Jiang, Y., J. D. Woronicz, et al. (1999). "Prevention of constitutive TNF receptor 1 signaling by silencer of death domains." Science 283(5401): 543-546.

Jimenez, C., C. Hernandez, et al. (2002). "The p85 regulatory subunit controls sequential activation of phosphoinositide 3-kinase by Tyr kinases and Ras." J Biol Chem 277(44): 41556-41562.

Jundi, M., A. Nadiri, et al. (2012). "CD40-mediated cell death requires TRAF6 recruitment." Immunobiology 217(3): 375-383. 
Kao, S., R. K. Jaiswal, et al. (2001). "Identification of the mechanisms regulating the differential activation of the mapk cascade by epidermal growth factor and nerve growth factor in PC12 cells." J Biol Chem 276(21): 18169-18177.

Kaplan, D. R., B. L. Hempstead, et al. (1991a). "The trk proto-oncogene product: a signal transducing receptor for nerve growth factor." Science 252(5005): 554-558.

Kaplan, D. R., D. Martin-Zanca, et al. (1991b). "Tyrosine phosphorylation and tyrosine kinase activity of the trk proto-oncogene product induced by NGF." Nature 350(6314): 158-160.

Katz, N., D. G. Borenstein, et al. (2011). "Efficacy and safety of tanezumab in the treatment of chronic low back pain." Pain 152(10): 2248-2258.

Kawabe, H. and N. Brose (2011). "The role of ubiquitylation in nerve cell development." Nat Rev Neurosci 12(5): 251-268.

Khursigara, G., J. R. Orlinick, et al. (1999). "Association of the p75 neurotrophin receptor with TRAF6." J Biol Chem 274(5): 2597-2600.

Kim, A. H., G. Khursigara, et al. (2001). "Akt phosphorylates and negatively regulates apoptosis signal-regulating kinase 1." Mol Cell Biol 21(3): 893-901.

Klein, R., V. Nanduri, et al. (1991). "The trkB tyrosine protein kinase is a receptor for brain-derived neurotrophic factor and neurotrophin-3." Cell 66(2): 395-403.

Knight, K. K., D. R. Olson, et al. (2006). "Liddle's syndrome mutations increase Na+ transport through dual effects on epithelial $\mathrm{Na}+$ channel surface expression and proteolytic cleavage." Proc Natl Acad Sci U S A 103(8): 2805-2808.

Kobayashi, K., T. Fukuoka, et al. (2005). "Distinct expression of TRPM8, TRPA1, and TRPV1 mRNAs in rat primary afferent neurons with adelta/c-fibers and colocalization with trk receptors." J Comp Neurol 493(4): 596-606.

Koltzenburg, M., D. L. Bennett, et al. (1999). "Neutralization of endogenous NGF prevents the sensitization of nociceptors supplying inflamed skin." Eur J Neurosci 11(5): 1698-1704.

Kramer, K., W. Gerald, et al. (1996). "Prognostic value of TrkA protein detection by monoclonal antibody 5C3 in neuroblastoma." Clin Cancer Res 2(8): 1361-1367.

Kuratomi, G., A. Komuro, et al. (2005). "NEDD4-2 (neural precursor cell expressed, developmentally down-regulated 4-2) negatively regulates TGF-beta (transforming growth factor-beta) signalling by inducing ubiquitin-mediated degradation of Smad2 and TGF-beta type I receptor." Biochem J 386(Pt 3): 461-470.

Kuruvilla, R., H. Ye, et al. (2000). "Spatially and functionally distinct roles of the PI3-K effector pathway during NGF signaling in sympathetic neurons." Neuron 27(3): 499-512.

Lane, N. E., T. J. Schnitzer, et al. (2010). "Tanezumab for the treatment of pain from osteoarthritis of the knee." N Engl J Med 363(16): 1521-1531.

Lee, F. S., A. H. Kim, et al. (2001a). "The uniqueness of being a neurotrophin receptor." Curr Opin Neurobiol 11(3): 281-286.

Lee, K. F., E. Li, et al. (1992). "Targeted mutation of the gene encoding the low affinity NGF receptor p75 leads to deficits in the peripheral sensory nervous system." Cell 69(5): 737-749.

Lee, R., P. Kermani, et al. (2001b). "Regulation of cell survival by secreted proneurotrophins." Science 294(5548): 1945-1948. 
Lemke, G. and M. Chao (1988). "Axons regulate Schwann cell expression of the major myelin and NGF receptor genes." Development 102(3): 499-504.

Levi-Montalcini, R. (1987). "The nerve growth factor 35 years later." Science 237(4819): 1154-1162.

Li, L., C. J. Xian, et al. (2003). "Lumbar 5 ventral root transection-induced upregulation of nerve growth factor in sensory neurons and their target tissues: a mechanism in neuropathic pain." Mol Cell Neurosci 23(2): 232-250.

Lindsay, R. M., C. Lockett, et al. (1989). "Neuropeptide expression in cultures of adult sensory neurons: modulation of substance $P$ and calcitonin gene-related peptide levels by nerve growth factor." Neuroscience 33(1): 53-65.

Lopes, C. M., J. I. Remon, et al. (2007). "Protein kinase A modulates PLC-dependent regulation and PIP2-sensitivity of K+ channels." Channels (Austin) 1(2): 124-134.

Maclnnis, B. L. and R. B. Campenot (2002). "Retrograde support of neuronal survival without retrograde transport of nerve growth factor." Science 295(5559): 1536-1539.

Makkerh, J. P., C. Ceni, et al. (2005). "p75 neurotrophin receptor reduces ligand-induced Trk receptor ubiquitination and delays Trk receptor internalization and degradation." EMBO Rep 6(10): 936-941.

Mamidipudi, V. and M. W. Wooten (2002). "Dual role for p75(NTR) signaling in survival and cell death: can intracellular mediators provide an explanation?" J Neurosci Res 68(4): 373-384.

Mantyh, P. W., M. Koltzenburg, et al. (2011). "Antagonism of nerve growth factor-TrkA signaling and the relief of pain." Anesthesiology 115(1): 189-204.

Marchese, A., C. Raiborg, et al. (2003). "The E3 ubiquitin ligase AIP4 mediates ubiquitination and sorting of the G protein-coupled receptor CXCR4." Dev Cell 5(5): 709-722.

Marmigere, F. and P. Ernfors (2007). "Specification and connectivity of neuronal subtypes in the sensory lineage." Nat Rev Neurosci 8(2): 114-127.

Martin-Zanca, D., M. Barbacid, et al. (1990). "Expression of the trk proto-oncogene is restricted to the sensory cranial and spinal ganglia of neural crest origin in mouse development." Genes Dev 4(5): 683-694.

Martin-Zanca, D., S. H. Hughes, et al. (1986). "A human oncogene formed by the fusion of truncated tropomyosin and protein tyrosine kinase sequences." Nature 319(6056): 743-748.

Matsuo, S., H. Ichikawa, et al. (2000). "Proprioceptive afferents survive in the masseter muscle of trkC knockout mice." Neuroscience 95(1): 209-216.

Mayers, J. R., I. Fyfe, et al. (2011). "ESCRT-0 assembles as a heterotetrameric complex on membranes and binds multiple ubiquitinylated cargoes simultaneously." $\underline{\mathrm{J} \text { Biol Chem }}$ 286(11): 9636-9645.

McCullough, J., P. E. Row, et al. (2006). "Activation of the endosome-associated ubiquitin isopeptidase AMSH by STAM, a component of the multivesicular body-sorting machinery." Curr Biol 16(2): 160-165.

McLeod, A. L., J. Ritchie, et al. (1999). "Transgenic mice over-expressing substance P exhibit allodynia and hyperalgesia which are reversed by substance $\mathrm{P}$ and N-methyl-D-aspartate receptor antagonists." Neuroscience 89(3): 891-899. 
McMahon, S. B., D. L. Bennett, et al. (1995). "The biological effects of endogenous nerve growth factor on adult sensory neurons revealed by a trkA-lgG fusion molecule." Nat Med 1(8): 774-780.

Meakin, S. O., J. I. MacDonald, et al. (1999). "The signaling adapter FRS-2 competes with Shc for binding to the nerve growth factor receptor TrkA. A model for discriminating proliferation and differentiation." J Biol Chem 274(14): 9861-9870.

Meller, R., M. Minami, et al. (2005). "CREB-mediated Bcl-2 protein expression after ischemic preconditioning." J Cereb Blood Flow Metab 25(2): 234-246.

Michael, G. J., S. Averill, et al. (1997). "Nerve growth factor treatment increases brain-derived neurotrophic factor selectively in TrkA-expressing dorsal root ganglion cells and in their central terminations within the spinal cord." J Neurosci 17(21): 8476-8490.

Michael, G. J. and J. V. Priestley (1999). "Differential expression of the mRNA for the vanilloid receptor subtype 1 in cells of the adult rat dorsal root and nodose ganglia and its downregulation by axotomy." J Neurosci 19(5): 1844-1854.

Mishra, S. K. and M. A. Hoon (2010). "Ablation of TrpV1 neurons reveals their selective role in thermal pain sensation." Mol Cell Neurosci 43(1): 157-163.

Mu, Y., R. Sundar, et al. (2011). "TRAF6 ubiquitinates TGFbeta type I receptor to promote its cleavage and nuclear translocation in cancer." Nat Commun 2: 330.

Mufson, E. J. and J. H. Kordower (1992). "Cortical neurons express nerve growth factor receptors in advanced age and Alzheimer disease." Proc Natl Acad Sci U S A 89(2): 569-573.

Mukai, J., T. Hachiya, et al. (2000). "NADE, a p75NTR-associated cell death executor, is involved in signal transduction mediated by the common neurotrophin receptor p75NTR." J Biol Chem 275(23): 17566-17570.

Mukhopadhyay, D. and H. Riezman (2007). "Proteasome-independent functions of ubiquitin in endocytosis and signaling." Science 315(5809): 201-205.

Myat, A., P. Henry, et al. (2002). "Drosophila Nedd4, a ubiquitin ligase, is recruited by Commissureless to control cell surface levels of the roundabout receptor." Neuron 35(3): 447-459.

Nakamura-Craig, M. and T. W. Smith (1989). "Substance P and peripheral inflammatory hyperalgesia." Pain 38(1): 91-98.

Namjou, B., C. B. Choi, et al. (2012). "Evaluation of TRAF6 in a large multi-ancestral lupus cohort." Arthritis Rheum.

Nealen, M. L., M. S. Gold, et al. (2003). "TRPM8 mRNA is expressed in a subset of cold-responsive trigeminal neurons from rat." $\underline{\mathrm{J} \text { Neurophysiol }}$ 90(1): 515-520.

Neuberg, M., J. Adamkiewicz, et al. (1989). "A Fos protein containing the Jun leucine zipper forms a homodimer which binds to the AP1 binding site." Nature 341(6239): 243-245.

Nicol, G. D. and M. R. Vasko (2007). "Unraveling the story of NGF-mediated sensitization of nociceptive sensory neurons: ON or OFF the Trks?" Mol Interv 7(1): 26-41.

Novick, P. and M. Zerial (1997). "The diversity of Rab proteins in vesicle transport." Curr Opin Cell Biol 9(4): 496-504.

Obata, K. and K. Noguchi (2006). "BDNF in sensory neurons and chronic pain." Neurosci Res 55(1): 1-10. 
Oku, R., M. Satoh, et al. (1987). "Release of substance $P$ from the spinal dorsal horn is enhanced in polyarthritic rats." Neurosci Lett 74(3): 315-319.

Otten, U., M. Goedert, et al. (1980). "Requirement of nerve growth factor for development of substance P-containing sensory neurones." Nature 287(5778): 158-159.

Paul, P. K., S. Bhatnagar, et al. (2012). "The E3 Ubiquitin Ligase TRAF6 Intercedes in Starvation-Induced Skeletal Muscle Atrophy through Multiple Mechanisms." Mol Cell Biol 32(7): 1248-1259.

Pecho-Vrieseling, E., M. Sigrist, et al. (2009). "Specificity of sensory-motor connections encoded by Sema3e-Plxnd1 recognition." Nature 459(7248): 842-846.

Peiro, S., J. X. Comella, et al. (2000). "PC12 cells have caveolae that contain TrkA. Caveolae-disrupting drugs inhibit nerve growth factor-induced, but not epidermal growth factor-induced, MAPK phosphorylation." J Biol Chem 275(48): 37846-37852.

Perl, E. R. (2007). "Ideas about pain, a historical view." Nat Rev Neurosci 8(1): 71-80.

Persaud, A., P. Alberts, et al. (2009). "Comparison of substrate specificity of the ubiquitin ligases Nedd4 and Nedd4-2 using proteome arrays." Mol Syst Biol 5: 333.

Pezet, S. and S. B. McMahon (2006). "Neurotrophins: mediators and modulators of pain." Annu Rev Neurosci 29: 507-538.

Pezet, S., B. Onteniente, et al. (2001). "Differential regulation of NGF receptors in primary sensory neurons by adjuvant-induced arthritis in the rat." Pain 90(1-2): 113-125.

Philippidou, P., G. Valdez, et al. (2011). "Trk retrograde signaling requires persistent, Pincher-directed endosomes." Proc Natl Acad Sci U S A 108(2): 852-857.

Raiborg, C. and H. Stenmark (2009). "The ESCRT machinery in endosomal sorting of ubiquitylated membrane proteins." Nature 458(7237): 445-452.

Rodriguez-Tebar, A., G. Dechant, et al. (1990). "Binding of brain-derived neurotrophic factor to the nerve growth factor receptor." Neuron 4(4): 487-492.

Rosenfeld, M. G., J. J. Mermod, et al. (1983). "Production of a novel neuropeptide encoded by the calcitonin gene via tissue-specific RNA processing." Nature 304(5922): 129-135.

Rothenberger, S., M. R. Food, et al. (1996). "Coincident expression and distribution of melanotransferrin and transferrin receptor in human brain capillary endothelium." Brain Res 712(1): 117-121.

Row, P. E., I. A. Prior, et al. (2006). "The ubiquitin isopeptidase UBPY regulates endosomal ubiquitin dynamics and is essential for receptor down-regulation." J Biol Chem 281(18): 12618-12624.

Ruiz, G., D. Ceballos, et al. (2004). "Behavioral and histological effects of endoneurial administration of nerve growth factor: possible implications in neuropathic pain." Brain Res 1011(1): 1-6.

Sah, D. W., M. H. Ossipo, et al. (2003). "Neurotrophic factors as novel therapeutics for neuropathic pain." Nat Rev Drug Discov 2(6): 460-472.

Salehi, A. H., P. P. Roux, et al. (2000). "NRAGE, a novel MAGE protein, interacts with the p75 neurotrophin receptor and facilitates nerve growth factor-dependent apoptosis." Neuron 27(2): 279-288.

Savaskan, E., F. Muller-Spahn, et al. (2000). "Alterations in trk A, trk B and trk C receptor immunoreactivities in parietal cortex and cerebellum in Alzheimer's disease." Eur Neurol 44(3): 172-180. 
Shailendra Kapoor, M. D. (2012). "TRPM8 antagonists and their emerging role in the modulation of pain and allodynia." Biochem Biophys Res Commun.

Shao, Y., W. Akmentin, et al. (2002). "Pincher, a pinocytic chaperone for nerve growth factor/TrkA signaling endosomes." J Cell Biol 157(4): 679-691.

Shelton, D. L. and L. F. Reichardt (1986). "Studies on the expression of the beta nerve growth factor (NGF) gene in the central nervous system: level and regional distribution of NGF mRNA suggest that NGF functions as a trophic factor for several distinct populations of neurons." Proc Natl Acad Sci U S A 83(8): 2714-2718.

Sigismund, S., T. Woelk, et al. (2005). "Clathrin-independent endocytosis of ubiquitinated cargos." Proc Natl Acad Sci U S A 102(8): 2760-2765.

Simons, K. and M. Zerial (1993). "Rab proteins and the road maps for intracellular transport." Neuron 11(5): 789-799.

Skoff, A. M. and J. E. Adler (2006). "Nerve growth factor regulates substance $P$ in adult sensory neurons through both TrkA and p75 receptors." Exp Neurol 197(2): 430-436.

Smeyne, R. J., R. Klein, et al. (1994). "Severe sensory and sympathetic neuropathies in mice carrying a disrupted Trk/NGF receptor gene." Nature 368(6468): 246-249.

Snider, W. D. (1994). "Functions of the neurotrophins during nervous system development: what the knockouts are teaching us." Cell 77(5): 627-638.

Sofroniew, M. V., N. P. Galletly, et al. (1990). "Survival of adult basal forebrain cholinergic neurons after loss of target neurons." Science 247(4940): 338-342.

Sommerfeld, M. T., R. Schweigreiter, et al. (2000). "Down-regulation of the neurotrophin receptor TrkB following ligand binding. Evidence for an involvement of the proteasome and differential regulation of TrkA and TrkB." J Biol Chem 275(12): 8982-8990.

Sorkin, A. and M. von Zastrow (2009). "Endocytosis and signalling: intertwining molecular networks." Nat Rev Mol Cell Biol 10(9): 609-622.

Starczynowski, D. T., W. W. Lockwood, et al. (2011). "TRAF6 is an amplified oncogene bridging the RAS and NF-kappaB pathways in human lung cancer." J Clin Invest 121(10): 4095-4105.

Staub, O., S. Dho, et al. (1996). "WW domains of Nedd4 bind to the proline-rich PY motifs in the epithelial $\mathrm{Na}+$ channel deleted in Liddle's syndrome." EMBO J 15(10): 2371-2380.

Stein, A. T., C. A. Ufret-Vincenty, et al. (2006). "Phosphoinositide 3-kinase binds to TRPV1 and mediates NGF-stimulated TRPV1 trafficking to the plasma membrane." $\underline{\mathrm{J} \text { Gen Physiol }}$ 128(5): 509-522.

Tai, H. C. and E. M. Schuman (2008). "Ubiquitin, the proteasome and protein degradation in neuronal function and dysfunction." Nat Rev Neurosci 9(11): 826-838.

Takahashi, Y., N. Shimokawa, et al. (2011). "Ligand-induced downregulation of TrkA is partly regulated through ubiquitination by Cbl." FEBS Lett 585(12): 1741-1747.

Tam, S. Y., M. Tsai, et al. (1997). "Expression of functional TrkA receptor tyrosine kinase in the HMC-1 human mast cell line and in human mast cells." Blood 90(5): 1807-1820.

Terenghi, G., D. Mann, et al. (1997). "trkA and trkC expression is increased in human diabetic skin." Neurosci Lett 228(1): 33-36.

Terrell, J., S. Shih, et al. (1998). "A function for monoubiquitination in the internalization of a G protein-coupled receptor." Mol Cell 1(2): 193-202. 
Tessarollo, L. (2001). "Manipulating mouse embryonic stem cells." Methods Mol Biol 158: 47-63.

Tsui-Pierchala, B. A. and D. D. Ginty (1999). "Characterization of an NGF-P-TrkA retrograde-signaling complex and age-dependent regulation of TrkA phosphorylation in sympathetic neurons." J Neurosci 19(19): 8207-8218.

Ugolini, G., S. Marinelli, et al. (2007). "The function neutralizing anti-TrkA antibody MNAC13 reduces inflammatory and neuropathic pain." Proc Natl Acad Sci U S A 104(8): 2985-2990.

US, V. E. and J. H. Gaddum (1931). "An unidentified depressor substance in certain tissue extracts." J Physiol 72(1): 74-87.

VanElzakker, M., R. D. Fevurly, et al. (2008). "Environmental novelty is associated with a selective increase in Fos expression in the output elements of the hippocampal formation and the perirhinal cortex." Learn Mem 15(12): 899-908.

Vega, J. A., E. Vazquez, et al. (1994). "Immunohistochemical localization of the high-affinity NGF receptor (gp140-trkA) in the adult human dorsal root and sympathetic ganglia and in the nerves and sensory corpuscles supplying digital skin." Anat Rec 240(4): 579-588.

Vellani, V., S. Mapplebeck, et al. (2001). "Protein kinase C activation potentiates gating of the vanilloid receptor VR1 by capsaicin, protons, heat and anandamide." J Physiol 534(Pt 3): 813-825.

Visser Smit, G. D., T. L. Place, et al. (2009). "Cbl controls EGFR fate by regulating early endosome fusion." Sci Signal 2(102): ra86.

Watson, F. L., H. M. Heerssen, et al. (2001). "Neurotrophins use the Erk5 pathway to mediate a retrograde survival response." Nat Neurosci 4(10): 981-988.

Wiley, H. S. and P. M. Burke (2001). "Regulation of receptor tyrosine kinase signaling by endocytic trafficking." Traffic 2(1): 12-18.

Wilkin, M. B., A. M. Carbery, et al. (2004). "Regulation of notch endosomal sorting and signaling by Drosophila Nedd4 family proteins." Curr Biol 14(24): 2237-2244.

Woelk, T., S. Sigismund, et al. (2007). "The ubiquitination code: a signalling problem." Cell Div 2: 11.

Wollert, T. and J. H. Hurley (2010). "Molecular mechanism of multivesicular body biogenesis by ESCRT complexes." Nature 464(7290): 864-869.

Woolf, C. J. (2000). "Pain." Neurobiol Dis 7(5): 504-510.

Wu, C., C. F. Lai, et al. (2001). "Nerve growth factor activates persistent Rap1 signaling in endosomes." J Neurosci 21(15): 5406-5416.

Yamashita, M., S. X. Ying, et al. (2005). "Ubiquitin ligase Smurf1 controls osteoblast activity and bone homeostasis by targeting MEKK2 for degradation." Cell 121(1): 101-113.

Yamashita, T., K. L. Tucker, et al. (1999). "Neurotrophin binding to the p75 receptor modulates Rho activity and axonal outgrowth." Neuron 24(3): 585-593.

Yang, B. and S. Kumar (2010). "Nedd4 and Nedd4-2: closely related ubiquitin-protein ligases with distinct physiological functions." Cell Death Differ 17(1): 68-77.

Yang, W. L., J. Wang, et al. (2009). "The E3 ligase TRAF6 regulates Akt ubiquitination and activation." Science 325(5944): 1134-1138. 
Ye, X., P. Mehlen, et al. (1999). "TRAF family proteins interact with the common neurotrophin receptor and modulate apoptosis induction." J Biol Chem 274(42): 30202-30208.

York, R. D., H. Yao, et al. (1998). "Rap1 mediates sustained MAP kinase activation induced by nerve growth factor." Nature 392(6676): 622-626.

Yu, T., L. Calvo, et al. (2011). "Regulation of trafficking of activated TrkA is critical for NGF-mediated functions." Traffic 12(4): 521-534.

Zhang, W., T. Na, et al. (2010). "Down-regulation of intestinal apical calcium entry channel TRPV6 by ubiquitin E3 ligase Nedd4-2." J Biol Chem 285(47): 36586-36596.

Zhang, X., J. Huang, et al. (2005). "NGF rapidly increases membrane expression of TRPV1 heat-gated ion channels." EMBO J 24(24): 4211-4223.

Zhang, Y., D. B. Moheban, et al. (2000). "Cell surface Trk receptors mediate NGF-induced survival while internalized receptors regulate NGF-induced differentiation." J Neurosci 20(15): 5671-5678.

Zhou, L. and H. Yang (2011). "The von Hippel-Lindau tumor suppressor protein promotes c-Cbl-independent poly-ubiquitylation and degradation of the activated EGFR." PLoS One 6(9): e23936.

Zhou, R., S. V. Patel, et al. (2007). "Nedd4-2 catalyzes ubiquitination and degradation of cell surface ENaC." J Biol Chem 282(28): 20207-20212.

Zucchelli, S., F. Marcuzzi, et al. (2011). "Tumor necrosis factor receptor-associated factor 6 (TRAF6) associates with huntingtin protein and promotes its atypical ubiquitination to enhance aggregate formation." J Biol Chem 286(28): 25108-25117.

Zweifel, L. S., R. Kuruvilla, et al. (2005). "Functions and mechanisms of retrograde neurotrophin signalling." Nat Rev Neurosci 6(8): 615-625. 


\section{Publications}




\section{Regulation of Trafficking of Activated TrkA Is Critical for NGF-Mediated Functions}

\author{
Tao Yu ${ }^{1}$, Laura Calvo ${ }^{1}$, Begoña Anta ${ }^{1}$, \\ Saray López-Benito ${ }^{1}$, Eileen Southon ${ }^{2}$, \\ Moses V. Chao ${ }^{3}$, Lino Tessarollo ${ }^{2}$ \\ and Juan C. Arévalo ${ }^{1, *}$
}

\begin{abstract}
${ }^{1}$ Department of Cell Biology and Pathology, Instituto de Neurociencias de Castilla y León (INCyL), Universidad de Salamanca, Salamanca 37007, Spain

${ }^{2}$ Neural Development Group, Mouse Cancer Genetics

Program, Center for Cancer Research, National Cancer Institute, Frederick, MD 21702, USA

${ }^{3}$ Molecular Neurobiology Program, Skirball Institute of Biomolecular Medicine, Departments of Cell Biology, Physiology and Neuroscience, Psychiatry, and Neural Sciences, New York University School of Medicine, New York, NY 10016, USA

*Corresponding author: Juan C. Arévalo,

arevalojc@usal.es
\end{abstract}

Upon activation by nerve growth factor (NGF), TrkA is internalized, trafficked and sorted through different endosomal compartments. Proper TrkA trafficking and sorting are crucial events as alteration of these processes hinders NGF-mediated functions. However, it is not fully known which proteins are involved in the trafficking and sorting of TrkA. Here we report that Nedd4-2 regulates the trafficking of TrkA and NGF functions in sensory neurons. Depletion of Nedd4-2 disrupts the correct sorting of activated TrkA at the early and late endosome stages, resulting in an accumulation of TrkA in these compartments and, as a result of the reduced trafficking to the degradative pathway, TrkA is either reverted to the cell surface through the recycling pathway or retrogradely transported to the cell body. In addition, Nedd4-2 depletion enhances TrkA signaling and the survival of NGF-dependent dorsal root ganglion neurons, but not those of brain-derived neurotrophic factor-dependent neurons. Furthermore, neurons from a knock-in mouse expressing a TrkA mutant that does not bind Nedd4-2 protein exhibit increased NGF-mediated signaling and cell survival. Our data indicate that TrkA trafficking and sorting are regulated by Nedd4-2 protein.

Key words: Nedd4-2, neurons, NGF, trafficking, TrkA

Received 22 September 2010, revised and accepted for publication 23 December 2010, uncorrected manuscript published online 24 December 2010, published online 1 February 2011

Neurotrophins are growth factors involved in different functions in the nervous system, including survival, proliferation, differentiation, axonal growth and synaptic plasticity. All neurotrophins, including nerve growth factor (NGF), brain-derived neurotrophic factor (BDNF), neurotrophin-3 (NT-3) and neurotrophin-4 (NT-4), exert their functions by binding to two different receptors, namely the neurotrophin receptors Trk, which belong to the receptor tyrosine kinase (RTK) superfamily, and the neurotrophin receptor $\mathrm{p} 75$, which belongs to the tumor necrosis factor receptor superfamily. All neurotrophins bind to p75 with similar affinity, whereas there is preferential binding to specific Trk receptors: NGF to TrkA, BDNF and NT-4 to TrkB and NT-3 to TrkC $(1,2)$.

The trafficking and sorting of activated RTKs is a complex and highly regulated process that is seminal for the regulation of the specificity and duration of RTK-generated responses (3-7). The first stage of RTKs upon internalization in response to ligand binding is the early endosome, where their cargo can follow different fates; receptors that are mainly recycled back to the plasma membrane (i.e. transferring receptor) are sent to recycling endosomes, whereas those destined mainly for degradation (i.e. the EGF receptor) are sorted to late endosomes and then to lysosomes (5). The trafficking of Trks is critical for neurotrophin-mediated functions. Trk proteins represent a class of RTKs that are both recycled and degraded upon neurotrophin-induced internalization (8-10). However, different Trks are differentially regulated; although TrkA is mainly recycled, TrkB is predominantly degraded $(11,12)$. In contrast to other RTKs, Trks also undergo retrograde transport from the tip of the axon to the soma, TrkA being the most widely studied neurotrophin receptor (13-19). Retrogradely transported TrkA modulates the survival of NGF-dependent neurons (13-19). Although the trafficking of activated TrkA upon NGF binding needs to be tightly regulated, little is known about the molecular machinery involved in the trafficking and sorting of activated TrkA.

The ubiquitination of RTKs has emerged as a critical event for appropriate receptor trafficking and degradation (20). Ubiquitination is a reversible protein modification that is achieved by two opposite families of enzymes: E3 ubiquitin ligases, which add the ubiquitin protein to other proteins, and deubiquitinases, which remove the ubiquitin (21). Three independent groups have reported the ubiquitination of Trk proteins (22-24). We have previously reported that TrkA, but not TrkB, binds through a PPXY motif and is ubiquitinated by Nedd4-2 (22), an E3 ubiquitin ligase that belongs to the family of Nedd4 E3 ubiquitin ligases (25). Over-expression of Nedd4-2 in heterologous cells leads specifically to the downregulation of TrkA protein without altering the internalization rate of the receptor (22). Additional support for our findings comes from an unbiased study using protein microarrays that identified TrkA as a binding partner of Nedd4-2 (26). Although it is 
clear that Nedd4-2 regulates the levels of TrkA, the mechanism by which Nedd4-2 exerts its function is unknown.

Here we report a new function for Nedd4-2 protein. In addition to modulating TrkA levels (22), Nedd4-2 regulates the trafficking of TrkA protein in response to NGF in dorsal root ganglion (DRG) neurons. We observed that Nedd4-2 depletion delays the arrival of active TrkA to late endosomes, but not to early endosomes, with a subsequent increase in the recycling and retrograde transport of TrkA. As a result, Nedd4-2 affects TrkA signaling and the survival of NGF-dependent sensory neurons; this is a specific effect because Nedd4-2 depletion does not alter the survival of BDNF-dependent DRG neurons. Furthermore, we have generated a knock-in mouse that expresses a TrkA mutant protein that does not bind Nedd4-2. Sensory neurons obtained from this knock-in show increased NGFmediated signaling and survival. Our data identify Nedd4-2 as a critical protein in the trafficking of TrkA and, therefore, in NGF-mediated functions.

\section{Results}

\section{Nedd4-2 regulates TrkA receptor levels}

To address whether the endogenous expression of Nedd4-2 modulates the levels of TrkA, we infected DRG neurons with lentivirus expressing specific shRNA to deplete Nedd4-2. Most DRG neurons express TrkA during development and depend on NGF for their survival (27), suggesting that these neurons offer an excellent model to study NGF and its receptor TrkA in vitro. Accordingly, we obtained DRG neurons from E15.5 rat embryos and cultured them in the presence of NGF $(50 \mathrm{ng} / \mathrm{mL})$ to select TrkA-expressing neurons. Then, the neurons were infected with control and Nedd4-2 shRNA lentivirus at day in vitro (DIV) 4 to modulate Nedd4-2 protein levels. The rate of infection to perform all the biochemical experiments reported here was at least $90 \%$, as seen from green fluorescent protein (GFP) expression (Figure S1). Cell lysates from infected neurons were subjected to western blot analysis to assess Nedd4-2 protein levels. Infection with Nedd4-2 shRNA lentivirus rendered a dramatic decrease in the levels of endogenous Nedd4-2 protein (Figure 1A). Concomitant with Nedd4-2 depletion, the amount of total TrkA was increased, whereas p75 levels were not affected (Figure 1A). To assess whether Nedd4-2 depletion might influence the surface expression of NGF receptors, we performed biotinylation experiments. Surface proteins from infected DRG neurons were labeled with biotin and pulled down with neutroavidin beads. Surface TrkA levels, but not surface p75 levels, were increased following Nedd4-2 depletion (Figure 1B). Quantification of the data corresponding to the different experiments indicated an $88 \%$ increase in total TrkA levels and a $46 \%$ increase in surface TrkA levels in Nedd4-2-depleted neurons as compared with the control neurons (Figure 1C). No significant differences in p75 levels were observed. These results suggest that endogenous Nedd4-2 in sensory neurons
A

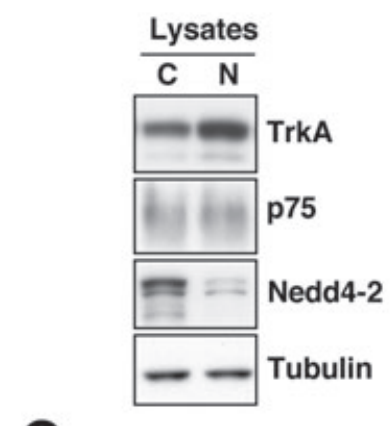

C

Avidin

pulldown

C $\mathrm{N}$

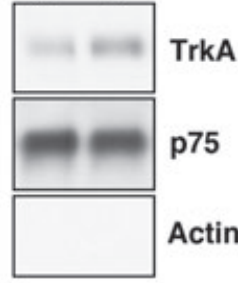

Actin

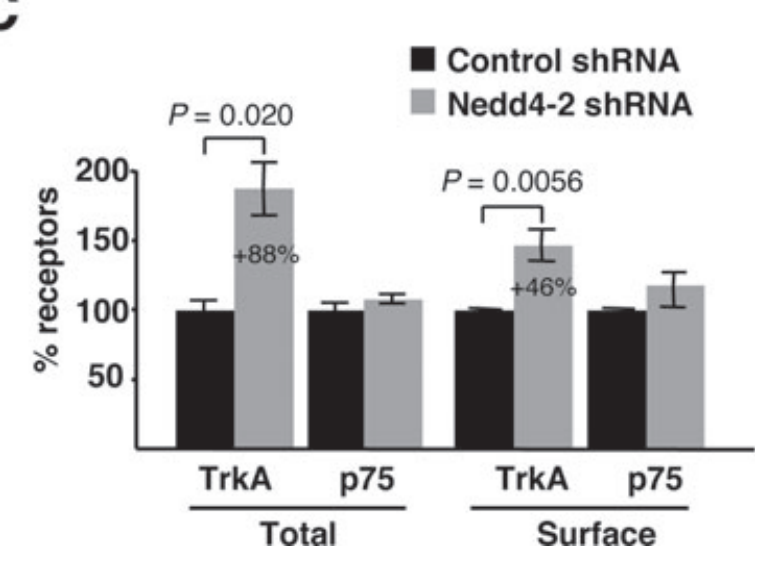

Figure 1: Nedd4-2 depletion increases TrkA levels. A) Increased levels of TrkA upon Nedd4-2 depletion. Cultured DRG neurons were infected on DIV4 with control or Nedd42 shRNA lentivirus and extracts were obtained on DIV10 to analyze the expression of different proteins using western blot analysis. Tubulin was used as a loading control. Representative western blots are shown. C, control shRNA lentivirus; N, Nedd4-2 shRNA lentivirus. B) Enhanced surface TrkA in Nedd4-2depleted neurons. Cell-surface proteins from NGF-starved DRG neurons infected with control and Nedd4-2 shRNA lentivirus were labeled with biotin and the same amount of proteins was pulled down with neutroavidin. Western blot analyses were performed with TrkA and p75 antibodies. Representative western blots are shown. C, control shRNA lentivirus; N, Nedd4-2 shRNA lentivirus. C) Quantification of TrkA and p75 proteins in response to Nedd42 depletion. Western blots were scanned and quantified using IMAGEJ software. Data are presented as means \pm SEM. p-Values were calculated using a two-tailed Student's $t$-test $(n=6-8)$.

regulates the amount of total and surface-bound TrkA, but not $\mathrm{p} 75$.

\section{Nedd4-2 modulates the degradation of TrkA in response to NGF}

Upon activation, TrkA receptors are ubiquitinated and terminated by degradation (22-24). To address whether Nedd4-2 modulates the degradation rate of TrkA, we performed degradation assays in response to NGF using biotinylation. NGF-dependent DRG neurons infected with control and Nedd4-2 shRNA lentivirus were NGF-starved overnight in the presence of the anti-apoptotic inhibitor 
A
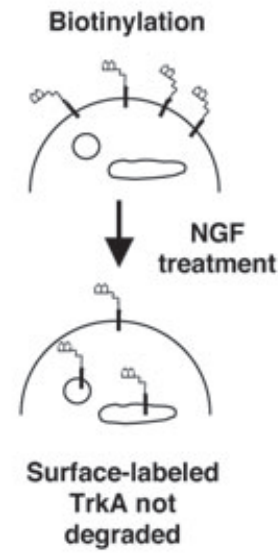

C

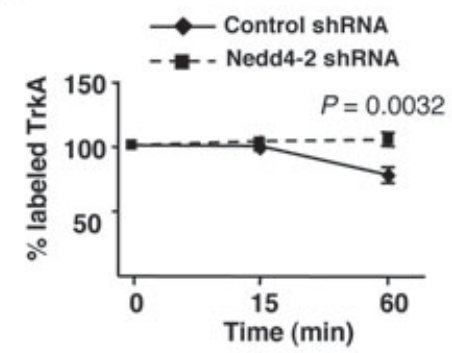

E

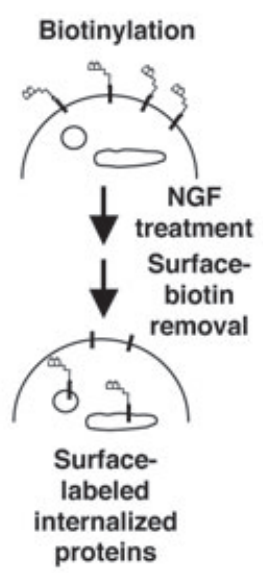

B

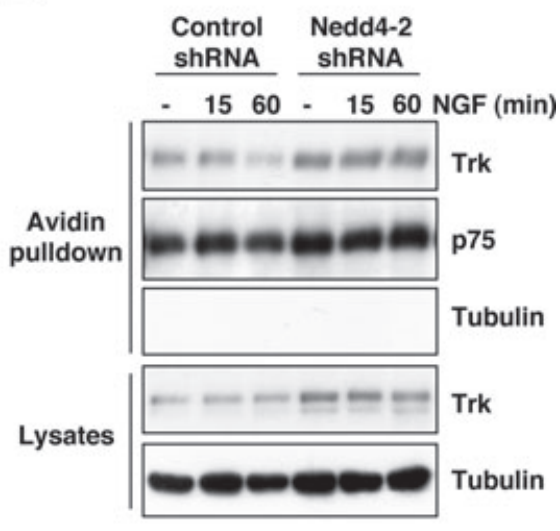

D

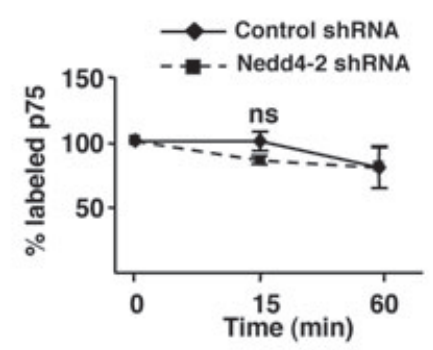

$\mathbf{F}$

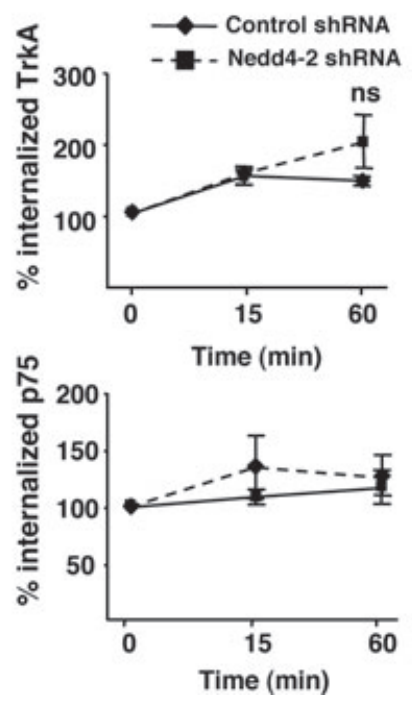

Figure 2: Nedd4-2 regulates TrkA degradation. A) A receptor degradation assay was performed as described in Materials and Methods and is depicted in the schematic diagram. Infected DRG neurons were biotinylated to label surface proteins and treated or not with NGF (50 ng/mL) for 15 and $60 \mathrm{~min}$. An aliquot of the cell lysates was used as loading control and the biotinylated proteins were precipitated with neutroavidin-agarose. Surface proteins were subsequently subjected to western blot analyses with Trk, p75 and tubulin antibodies. B) Representative western blots are shown. Tubulin was used as a negative control for biotinylated proteins and as a loading control for lysates. C) Quantification of surface-labeled TrkA. Data are normalized to the amount of biotinylated TrkA in control and in Nedd4-2depleted neurons without NGF treatment. Data are presented as means \pm SEM. p-Values were calculated using two-tailed Student's $t$-test $(n=4)$. D) Quantification of surface-labeled p75. Data are normalized to the amount of biotinylated p75 in control and in Nedd4-2depleted neurons without NGF treatment. Data are presented as means $\pm \operatorname{SEM}(n=4) ; \mathrm{ns}$, not significant. E) A schematic diagram of the internalization assay using biotinylation procedure is shown. DRG neurons were infected with control and Nedd4-2 shRNA lentivirus. Cells were biotinylated as described in Experimental Procedures and then treated with NGF ( $50 \mathrm{ng} / \mathrm{mL}$ ) for 15 and $60 \mathrm{~min}$. Biotin from non-internalized proteins was removed using reducing conditions as described in Materials and Methods. Cell lysates were prepared, surface proteins were subjected to precipitation with neutroavidin and western blot analyses were performed with the corresponding antibodies. Tubulin was used as a negative control for biotinylated proteins and as a loading control for lysates. F) Quantification of internalized TrkA receptor. Data are presented as average $\pm \operatorname{SEM}(n=3)$; ns, not significant. G) Quantification of internalized p75 receptor. Data are presented as average $\pm \operatorname{SEM}(n=3)$.
Z-VAD-FMK, and then the cell-surface proteins were biotinylated, followed by NGF stimulation $(50 \mathrm{ng} / \mathrm{mL})$, as described in Materials and Methods and outlined in Figure 2A. After $1 \mathrm{~h}$ of NGF treatment, there was a clear decrease in the amount of biotin-labeled TrkA in the control neurons as a result of TrkA degradation (Figure 2B). However, the amount of biotinylated TrkA receptors from Nedd4-2-depleted neurons was not altered (Figure 2B). Quantification of multiple experiments indicated no significant degradation of surface TrkA in Nedd4-2-depleted neurons upon NGF stimulation for $1 \mathrm{~h}$, whereas a $30 \%$ reduction in surface TrkA was noted in the control cells (Figure 2C). The levels of biotinylated TrkA were similar in the control and Nedd4-2 shRNA neurons after 15 min of NGF treatment (Figure 2B,C). No differences were detected in the degradation rate of $\mathrm{p} 75$ in response to NGF from the control and Nedd4-2-depleted neurons (Figure 2B,D). Therefore, Nedd4-2 levels modulate the degradation rate of TrkA in response to NGF.

The degradation of TrkA protein in response to NGF requires the internalization and trafficking of the receptor 
to the lysosomes. To assess whether Nedd4-2 protein modulates the internalization rate of TrkA in response to NGF, we performed internalization assays in infected DRG neurons using biotinylation. Neurons were NGF-starved as described above and cell-surface biotinylation was performed using cleavable biotin, as outlined in Figure 2E. NGF $(50 \mathrm{ng} / \mathrm{mL}$ ) was then applied for 15 or 60 min and reducing conditions were applied to strip the biotin from surface proteins that had not been internalized. This treatment allows the detection of internalized biotinylated proteins, which remained protected from cleavage, upon pulling them down using neutroavidin beads. We did not observe any significant differences in the rate of internalization of TrkA or p75 in the control and Nedd4-2-depleted cells (Figure 2F,G). Thus, these data suggest that Nedd4-2 protein does not regulate the internalization rate of TrkA in response to NGF. These results are consistent with previous observations regarding the lack of differences in the internalization rate of wild-type TrkA and TrkAP782S, a mutant receptor that does not bind Nedd4-2 (22).

\section{TrkA trafficking at early and late endosomes is regulated by Nedd4-2}

To address whether Nedd4-2 regulates TrkA trafficking, we performed immunofluorescence analysis to detect the localization of activated TrkA upon depletion of Nedd4-2 in sensory neurons. First, we identified the localization of Nedd4-2 in neurons using a previously described antibody (22). Staining of NGF-dependent DRG neurons indicated a strong colocalization of Nedd4-2 with the early endosomal markers EEA1 and Rab5 and with the late endosomal marker Rab7 (Figure 3A). This localization resembles the localization of Itch, another member of the Nedd4 family $(28,29)$. The specificity of Nedd4-2 antibody was confirmed using Nedd4-2-depleted neurons, which showed almost no signal from the Nedd4-2 antibody as compared with the control neurons (Figure S2). These data indicate that Nedd4-2 is located in early and late endosomes.

To address whether Nedd4-2 modulates the location of activated TrkA, we performed stainings with antibodies against phosphorylated TrkA $(22,30)$ and different endosomal markers. Infected NGF-dependent neurons were starved as described above, stimulated with NGF ( $50 \mathrm{ng} / \mathrm{mL}$ ) for different times and stained. Upon NGF treatment, internalized TrkA colocalized with Rab5 in both the control and Nedd4-2-depleted cells (Figure 3B). No staining with pTrkA antibody was observed in the absence of NGF (data not shown). To quantify the colocalization, we used a custom program, as described in Materials and Methods, and the data corresponding to the surface levels of TrkA at steady state in control and Nedd4-2-depleted neurons were normalized. No significant differences in the colocalization of pTrkA with Rab5 were observed between the control and Nedd4-2-depleted neurons at 5 or $30 \mathrm{~min}$ after normalization (Figure $3 \mathrm{C}$ ). Therefore, these results indicate that depletion of Nedd4-2 does not alter the arrival of activated TrkA at early endosomes, in support of previous data indicating that Nedd4-2 does not modify the internalization of TrkA in response to NGF.

In addition, we performed immunofluorescence studies of activated TrkA with the late endosomal marker Rab7 in response to Nedd4-2 depletion (Figure 3D). Our data normalized as stated above indicated that after $10 \mathrm{~min}$ of NGF treatment there was a $40 \%$ decrease in the amount of pTrkA present in the late endosomes of Nedd4-2depleted cells (Figure 3E). However, at later time-points along NGF treatment (30 min) there was a massive accumulation of pTrkA receptors in late endosomes from Nedd4-2-depleted neurons as compared with the control neurons (300\% increase) (Figure 3E). Accordingly, the sorting of activated TrkA between early and late endosomes and between late endosomes and lysosomes seems to be regulated by Nedd4-2. These data were further confirmed using a biochemical approach. Neurons infected with control and Nedd4-2 shRNA lentivirus were NGF-starved overnight, followed by stimulation with NGF (50 ng/mL) for 10 or $30 \mathrm{~min}$ and the cells were homogenized without detergent to obtain crude membranes. Rab5 and Rab7 antibodies were used to immunoprecipitate the same amount of endomembranes containing these two proteins from control and Nedd4-2-depleted neurons treated with NGF for 10 min (Rab5 immunoprecipitation) or for 30 min (Rab7 immunoprecipitation) and the amount of TrkA protein in the immunoprecipitated membranes was assessed. An increase in TrkA in Rab5and Rab7-endomembranes from Nedd4-2-depleted neurons as compared with the control neurons was observed after NGF treatment for 10 and $30 \mathrm{~min}$, respectively (Figure 3F). Taken together, the colocalization and the biochemical experiments suggest that Nedd4-2 regulates the trafficking of activated TrkA at the level of early and late endosomes.

To address whether Nedd4-2 depletion altered specifically the ubiquitination status of TrkA in early endosomes, we performed immunoprecipitation of activated TrkA from Rab5-immunoprecipitated endomembranes. Western blots using ubiquitin antibodies indicated that TrkA ubiquitination was reduced in Nedd4-2-depleted neurons (Figure 3G). Therefore, these data indicate that Nedd42 ubiquitinates TrkA at the early endosomes, further supporting the role of Nedd4-2 protein in these compartments.

\section{Increased TrkA recycling to the plasma membrane by Nedd4-2 depletion}

Activated TrkA that reaches early endosomes can also recycle to the plasma membrane via recycling endosomes (31). To address whether Nedd4-2 modulates the recycling of activated receptors to the plasma membrane, we performed immunofluorescence analysis in DRG neurons using the recycling endosomal marker Rab11. Our data suggested that there might be more colocalization of pTrkA with recycling endosomes in Nedd4-2-depleted neurons than in control neurons (Figure 4A). Indeed, 
A
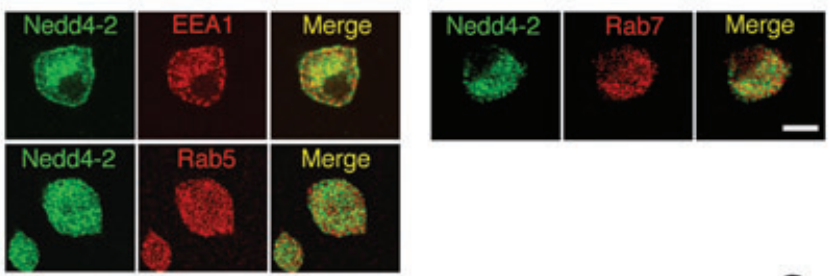

B

NGF 5 min

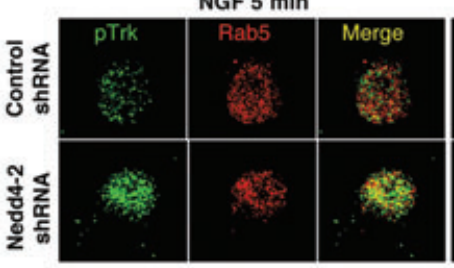

C

D

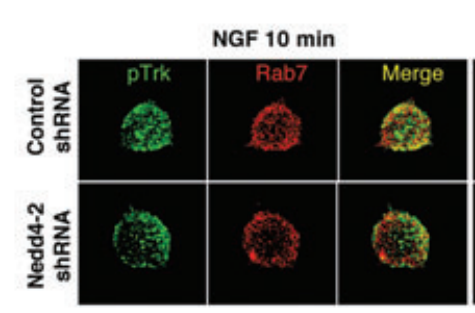

F

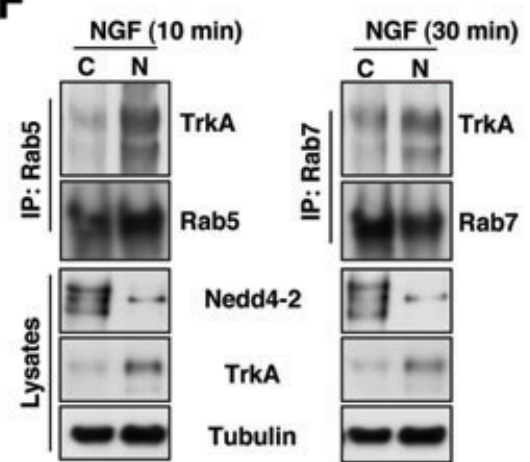

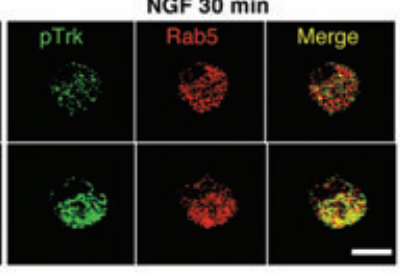

G

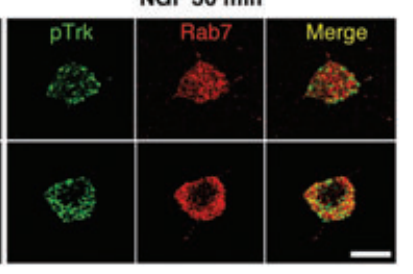

E
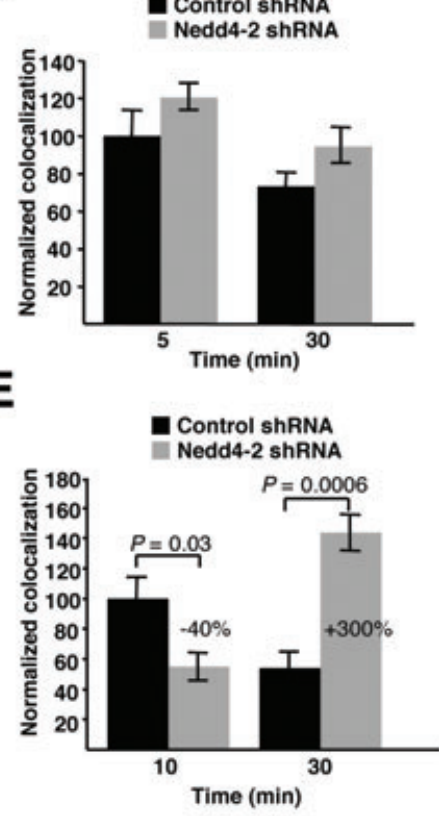

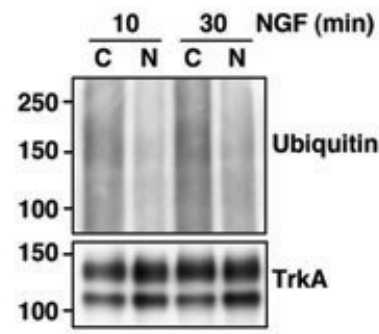

1: IP: Rab5

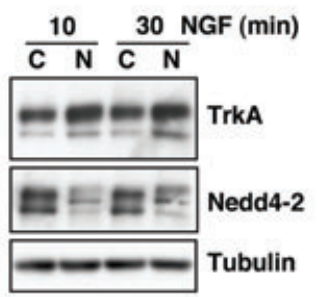

Lysates
2: IP: TrkA

Figure 3: Upon NGF treatment pTrkA localization is regulated by Nedd4-2. A) Colocalization of Nedd4-2 with early (EEA1 and Rab5) and late endosomes (Rab7). Scale bar, $20 \mu \mathrm{m}$. B) Colocalization of pTrkA with Rab5 compartments upon NGF treatment in control and in Nedd4-2 shRNA-infected DRG neurons. Infected DRG neurons were identified by GFP expression (data not shown) as described in Figure S1. Immunofluorescence was performed as described in Materials and Methods. Images were taken with a confocal microscope. Scale bar, $20 \mu \mathrm{m}$. C) Quantification of pTrkA colocalization in Rab5 endosomes. Images were processed using IMAGEJ and the percentage of colocalization was normalized to the surface levels of TrkA in control and in Nedd4-2-depleted neurons in the absence of NGF. Data are presented as means \pm SEM ( $n=6-9$ neurons/time-point). D) Colocalization of pTrkA with Rab7 compartments upon NGF treatment in control and in Nedd4-2 shRNA-infected DRG neurons was performed as described in Figure 3B. Scale bar, $20 \mu \mathrm{m}$. E) Quantification of pTrkA colocalization in Rab7 endosomes was performed as described in Figure 3C. Data are presented as means \pm SEM. p-Values were calculated using a two-tailed Student's $t$-test ( $n=5-7$ neurons/time-point). F) Immunoprecipitation of Rab5- and Rab7-endomembranes. Infected DRG neurons were treated with NGF for 10 or 30 min and lysates were immunoprecipitated with Rab5 or Rab7 antibodies, respectively. Representative blots are shown ( $n=3)$. C, control shRNA lentivirus; N, Nedd4-2 shRNA lentivirus. G) Reduced ubiquitination of activated TrkA in endosomal compartments. Infected DRG neurons were homogenized, immunoprecipitated with Rab5 antibodies and, after solubilization, immunoprecipitated with TrkA antibodies. Representative blots are shown $(n=2)$. C, control shRNA lentivirus; N, Nedd4-2 shRNA lentivirus. 
Yu et al.

A

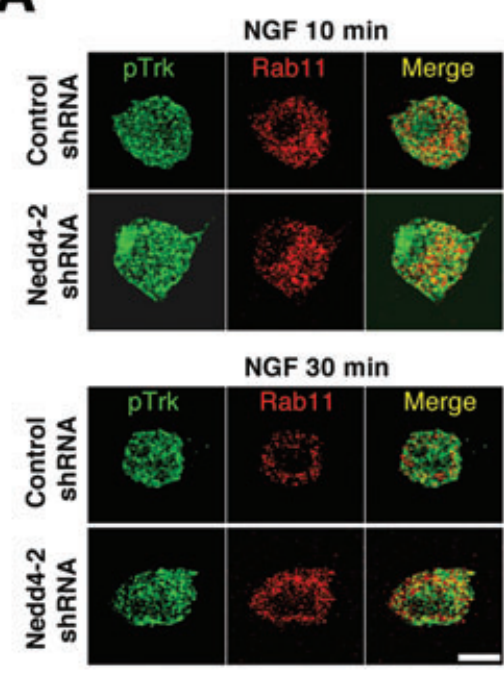

C

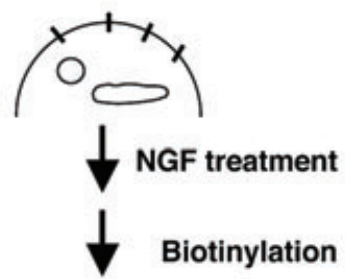

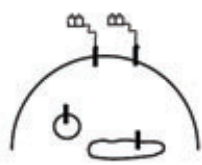

Surface-labeled proteins

E

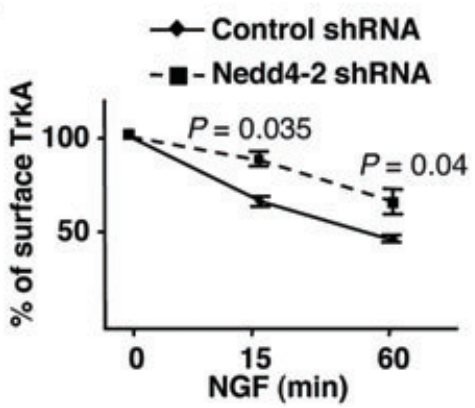

B

Control shRNA

- Nedd4-2 shRNA

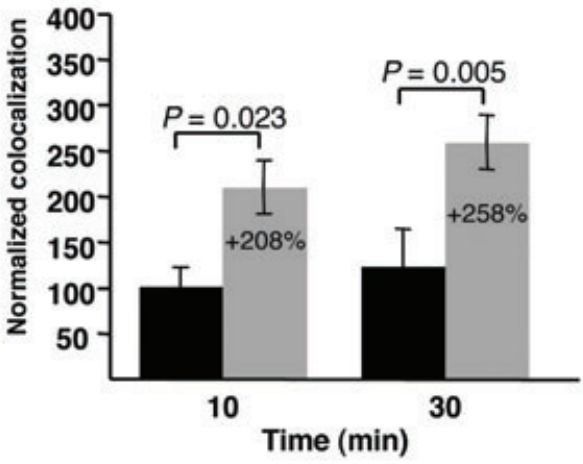

D

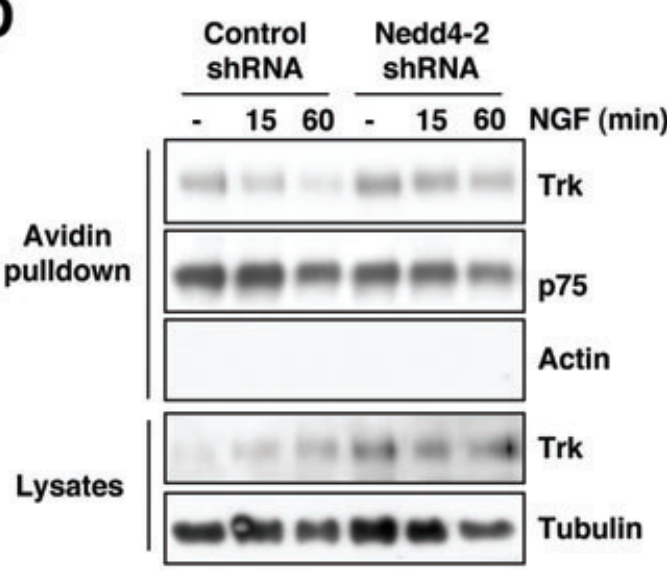

$\mathbf{F}$

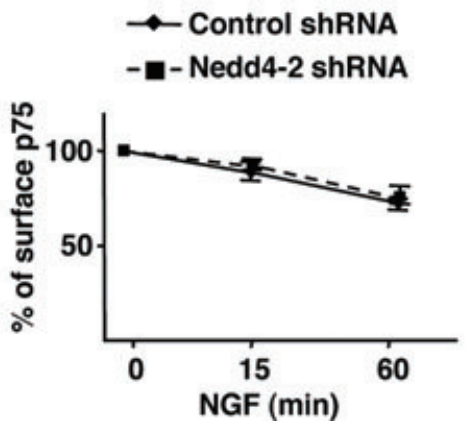

Figure 4: Enhanced pTrkA recycling and surface expression of TrkA in Nedd4-2-depleted DRG neurons. A) Colocalization of $p$ TrkA with Rab11 compartments upon NGF treatment in control and in Nedd4-2 shRNA-infected DRG neurons was performed as described in Figure 3B. Scale bar, $20 \mu \mathrm{m}$. B) Quantification of pTrkA colocalization in Rab11 endosomes was done as described in Figure 3C. Data are presented as means \pm SEM. $p$-Values were calculated using a two-tailed Student's $t$-test ( $n=5-7$ neurons/time-point). C) A schematic diagram of the surface expression assay using the biotinylation procedure in response to NGF is shown. DRG neurons were infected with control and Nedd4-2 shRNA lentivirus. Cells were treated with NGF (50 ng/mL) for 15 and 60 min and then biotinylated as described in Materials and Methods. Cell lysates were prepared, surface proteins were subjected to precipitation with neutroavidin-agarose and western blot analyses were performed with the corresponding antibodies. D) Representative western blots are shown. Tubulin and actin were used as a loading control for lysates and as a negative control for biotinylated proteins, respectively. E) Quantification of surface TrkA upon NGF treatment. Data are presented as means \pm SEM. p-Values were calculated using a two-tailed Student's $t$-test $(n=3)$. F) Quantification of surface p75 upon NGF treatment. Data are presented as means $\pm \operatorname{SEM}(n=3)$. 
quantification analyses of different cells indicated that in Nedd4-2-depleted neurons there was an increased colocalization of pTrkA with Rab11 as compared with the control neurons $(208 \%$ at $10 \mathrm{~min}$ and $258 \%$ at $30 \mathrm{~min}$ ) (Figure 4B). These data suggest that more TrkA protein recycles to the cell surface when Nedd4-2 is reduced. Further confirmation for this hypothesis came from biotinylation experiments aimed at detecting the amount of TrkA at the cell surface at different time-points upon NGF treatment, as outlined in Figure 4C. Upon NGF treatment $(50 \mathrm{ng} / \mathrm{mL})$, we observed a reduction over time in the amount of surface TrkA in control cells (Figure 4D,E). However, in Nedd4-2-depleted neurons this reduction in TrkA surface levels in response to NGF stimulation was attenuated (Figure 4D,E), suggesting that TrkA recycling may be enhanced. In contrast, the reduction in p75 from the cell surface in response to NGF was not affected by Nedd4-2 depletion (Figure 4D,F). Therefore, it may be surmised that TrkA recycles more and is increased in the plasma membrane when Nedd4-2 is depleted, further supporting a potential role for Nedd4-2 in TrkA sorting at early endosomes.

\section{Nedd4-2 depletion enhances the retrograde transport of TrkA in DRG neurons}

An additional fate of internalized TrkA protein that escapes degradation is retrograde transport to the cell body (19). To address whether the depletion of Nedd4-2 affected the retrograde transport of activated TrkA, we set up compartmentalized NGF-dependent DRG cultures using microfluidic compartments. The rate of retrograde transport in neurons was monitored by the uptake and retrograde transport of red-fluorescent beads by healthy neurons projecting axons to the beads-containing distal compartment (Figure 5A). These beads do not bind to TrkA protein. DRG neurons were infected with lentivirus expressing control or Nedd4-2 shRNA on DIV4, NGFstarved overnight at DIV7 and then stimulated with NGF $(50 \mathrm{ng} / \mathrm{mL})$ at the distal axons for $15 \mathrm{~h}$. Nedd4-2 depletion resulted in an increased amount of activated TrkA in the cell body compartment as compared with the control neurons (Figure 5B). Therefore, the retrograde transport of activated TrkA is also affected by Nedd4-2 protein.

\section{Nedd4-2 depletion increases TrkA signaling and the survival of NGF-dependent DRG neurons}

What are the functional consequences of Nedd4-2 depletion on DRG neurons? To address whether TrkA-mediated signaling was affected by Nedd4-2 expression, we infected NGF-dependent DRG neurons with control and Nedd4-2 shRNA lentivirus and performed western blot analysis in response to NGF. Infected neurons were NGFstarved overnight in the presence of the anti-apoptotic inhibitor Z-VAD-FMK, after which NGF $(50 \mathrm{ng} / \mathrm{mL})$ was applied for different times. Cell lysates were collected and analyzed by western blot using antibodies that recognized activated forms of TrkA, phospholipase $\mathrm{C} \gamma(\mathrm{PLC} \gamma)$, Akt and
A

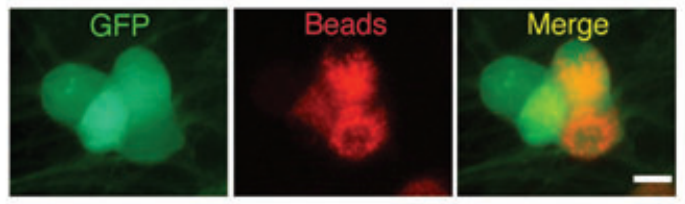

B

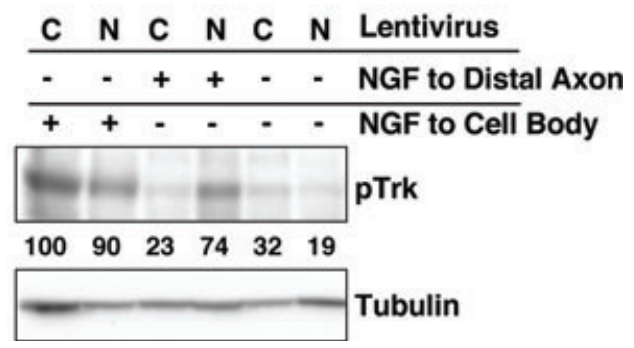

Figure 5: Enhanced NGF-mediated retrograde transport of pTrkA in Nedd4-2-depleted DRG neurons. A) DRG neurons growing in microfluidic chambers. Retrograde transport in infected neurons (GFP positive) was monitored by the presence of red fluorescence in the cell body compartment. Red-fluorescent beads were added to the distal compartment $15 \mathrm{~h}$ before subsequent experiments were performed. Scale bar, $20 \mu \mathrm{m}$. B) Increased retrograde transport of pTrkA upon Nedd4-2 depletion. Neurons were infected with control or Nedd4-2 shRNA lentivirus and either not stimulated or stimulated with NGF for $10 \mathrm{~min}$ in the cell body compartment or for $15 \mathrm{~h}$ in the distal compartment. Lysates were analyzed by western blot with pTrkA antibodies. Tubulin was used as a loading control. Numbers represent pTrk intensity normalized with respect to tubulin loading. C, control shRNA lentivirus; N, Nedd4-2 shRNA lentivirus.

MAPK. Our results indicate that the depletion of Nedd42 resulted in an enhancement of the phosphorylation of TrkA and, subsequently, the PLC $\gamma$, Akt and MAPK signaling pathways (Figure 6A). Thus, these data suggested that Nedd4-2 regulates NGF-mediated signaling.

DRG neurons depend on neurotrophins through Trkmediated signaling for their survival during development (27). To address whether Nedd4-2 modulates the dependence of sensory neuron survival on neurotrophins, we obtained NGF- and BDNF-dependent neurons and modified their levels of Nedd4-2. The dissected neurons were transfected on DIV4-5 with plasmids that expressed GFP and control shRNA or GFP and Nedd4-2 shRNA. The presence of GFP allows transfected neurons to be identified unambiguously. Apoptotic and non-apoptotic cells were scored at $72-120 \mathrm{~h}$ after NGF or BDNF withdrawal (Figure 6B). Non-transfected neurons showed approximately $60 \%$ of apoptosis upon neurotrophin deprivation for $72-120 \mathrm{~h}$ (data not shown). The percentage of apoptosis observed upon NGF withdrawal in the Nedd42-depleted neurons was only $34.9 \%$, i.e. significantly reduced in comparison with $60.8 \%$ of control transfected neurons (Figure 6C). Upon BDNF deprivation, no differences in apoptosis between control (58.7\%) and 
A

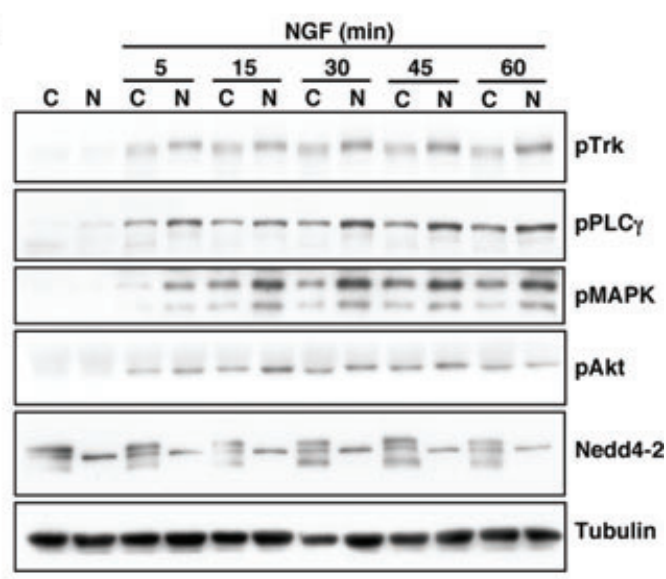

B

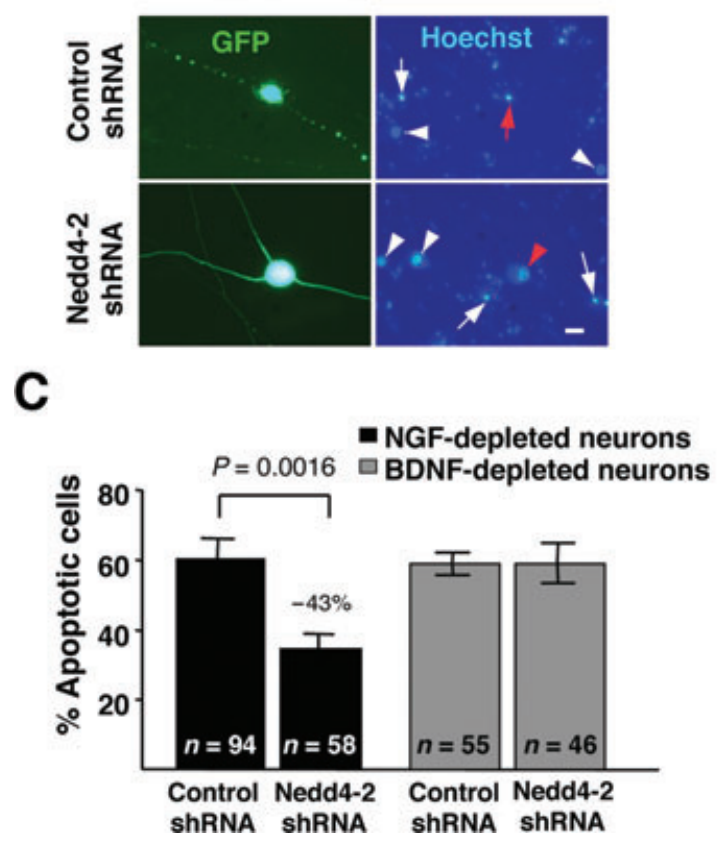

Figure 6: Nedd4-2 modulates NGF-mediated signaling and cell survival. A) Nedd4-2 depletion enhances NGF-mediated signaling. DRG neurons were infected with control and Nedd4-2 shRNA lentivirus, and cell extracts were analyzed upon NGF treatment. Active TrkA, PLC $\gamma$, MAPK and Akt were assessed using antibodies that recognized specific phosphorylated residues. Representative blots are shown $(n=5)$. C, control shRNA lentivirus; N, Nedd4-2 shRNA lentivirus. B) Images showing NGF-dependent DRG neurons transfected with control and Nedd4-2 shRNA plasmids. Transfected cells were visualized by GFP expression, and non-apoptotic cells (arrowheads) and apoptotic cells (arrows) were identified by staining with Hoechst 3342. Transfected neurons in the Hoechst 3342 panel are indicated in red. Scale bar, $20 \mu \mathrm{m}$. C) Quantification of apoptosis in NGF- and BDNF-dependent neurons transfected with control and Nedd4-2 shRNA plasmids upon withdrawal of the corresponding neurotrophin for 72-120 h. Data are presented as means \pm SEM. p-Values were calculated using a two-tailed Student's $t$-test [ $n$, number of cells from six (NGF) and three (BDNF) independent experiments].
Nedd4-2 shRNA (58.6\%) transfected cells were observed in BDNF-dependent neurons (Figure 6C). Therefore, these data indicate that Nedd4-2 is able to specifically influence the survival of NGF-dependent sensory neurons through the modulation of NGF-mediated signaling.

\section{Enhanced TrkA signaling and survival in DRG neurons from TrkAP782S mutant mice}

As Nedd4-2 can ubiquitinate several different proteins (32), shRNA could exert multiple direct and indirect effects on TrkA protein. To rule out the possibility that our results might be the consequence of an indirect effect, we followed a different experimental approach. Thus, we disrupted the binding of Nedd4-2 to TrkA. Previously, we have reported that the PPVY785 motif present in TrkA, but not in TrkB, is required for the binding of Nedd4-2 (22). Therefore, we generated a knock-in mouse carrying a point mutation in TrkA protein in the codon that encodes for proline 782, which was replaced by serine (Figure 7A-D), the amino acid present in TrkB. This mutant TrkA protein (TrkAP782S) lacks the PPVY motif and does not bind Nedd4-2 (22), but is still able to engage PLC $\gamma$ (Figure S3). The targeting vector was constructed by introducing two changes in the proline 782 codon to code for serine in exon 17 (Figure 7A). The presence of these changes was confirmed by sequencing and by the disappearance of a BstXI restriction site. Targeted embryonic stem (ES) cells were monitored by Southern blot with an external probe (Figure 7B) and with an internal (neo) probe (data not shown), as described in Materials and Methods. Knock-in TrkAP782S mice (KI) were viable and fertile with a normal life span. Genotyping of the mice was performed by polymerase chain reaction (PCR), amplifying 1185 and $1385 \mathrm{bp}$ fragments from the wild-type and $\mathrm{KI}$, respectively (Figure $7 \mathrm{C}$ ). The presence of the mutation was assessed by the lack of digestion of the PCR fragment with BstXI in the KI mice (Figure 7D). To confirm that the mutant-amplified DNA could be digested, we used the Pstl restriction site (Figure 7D).

To assess whether the NGF-mediated signaling emanating from TrkAP782S protein was altered, we obtained DRG neurons from E13.5 WT and KI embryos. Neurons were cultured and processed as described previously for rat DRG neurons. NGF-dependent neurons from KI mice did present an increase in TrkA, PLC $\gamma$, Akt and MAPK activation as compared with the WT neurons (Figure 7E). Therefore, TrkAP782S protein, which does not bind Nedd4-2, is more active upon NGF binding, leading to a higher activation of the downstream signaling cascades.

During development, DRG neurons project to their targets and receive trophic support from the neurotrophins provided by the tissues innervated. Neurons that do not receive enough signaling will die (27). To address the functional consequences of TrkAP782S expression in DRGs, we performed survival assays in NGF-dependent neurons obtained from WT and KI mice. Dissected neurons were plated on different amounts of $\operatorname{NGF}(25,20,5$ and 
A

B

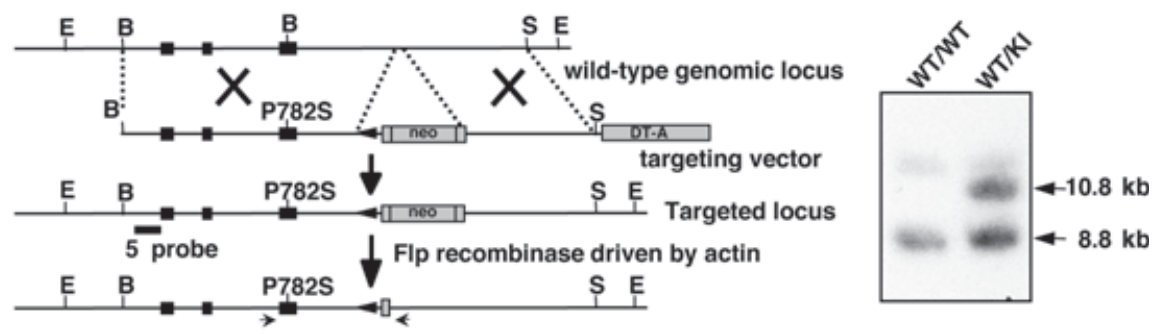

C
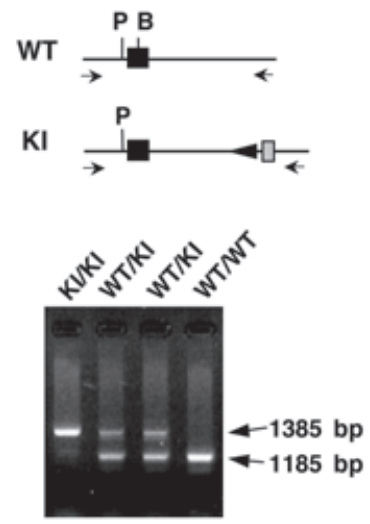

E

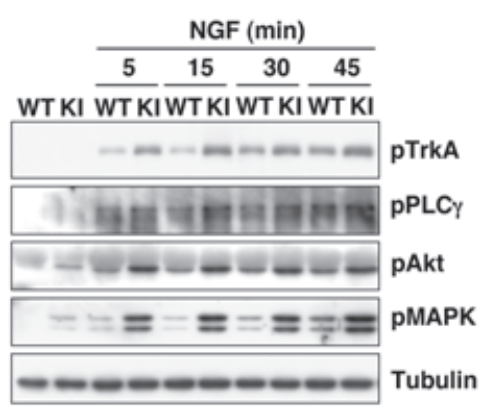

D

\begin{tabular}{c|c|c|c|} 
& PCR fragment & BstXI digestion & Pstl digestion \\
\hline WT & 1185 & $1016+169$ & $1041+144$ \\
\hline KI & 1385 & 1385 & $1241+144$ \\
\hline
\end{tabular}

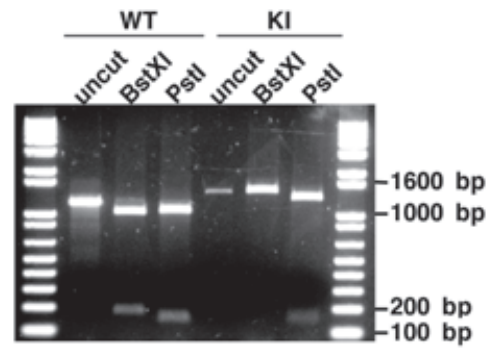

$\mathbf{F}$

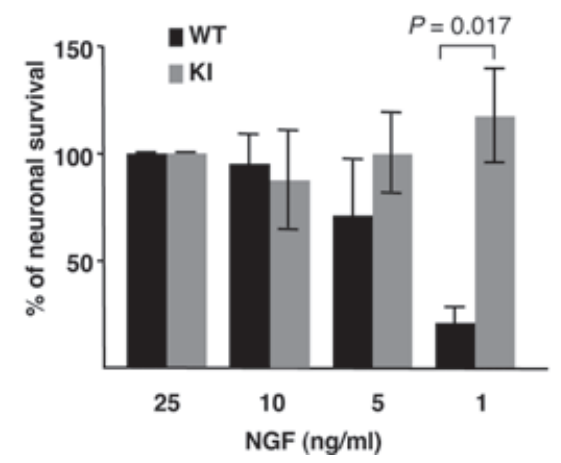

Figure 7: DRG neurons from TrkAP782S mouse display enhanced NGF-mediated signaling and survival. A) Schematics of the wild-type TrkA allele, the targeting vector and the targeted allele before and after Flp recombination. Neo, neomycin-resistance gene; DT-A, diphtheria toxin. E, B and S denote the sites for the restriction enzymes EcoRV, BstXI and Smal; black rectangles denote exons; triangles denote loxP sites and gray rectangles flanking Neo cassette denote FRT sites. B) Southern blot analysis of genomic DNA from WT and targeted ES cells after digestion with EcoRV. As indicated in the schematics of (A), the $5^{\prime}$ probe detects an 8.8-kb fragment from WT alleles and a 10.8-kb fragment from the targeted ES cells. C) PCR genotyping using mouse tail DNAs is shown. The primer sites used for genotyping are shown (arrows). B, BstXI; P, Pstl. D) The presence of the mutation that changes the proline to alanine was assessed by Bst XI digestion in the PCR product obtained from the KI animals. The upper panel shows the expected DNA fragments from digestion of the amplified DNA from the WT and KI mice. Note the lack of digestion with BstXI, but not with Pstl, in the amplified DNA fragment from the KI mouse. E) Enhanced NGF-mediated signaling in DRG neurons from TrkAP782S mice. DRG neurons were obtained and cultured for $6-8$ days, as described in Materials and Methods. NGF was withdrawn and Z-VAD-FMK (20 $\mu$ M) was added to the neurons overnight. Stimulation with NGF $(50 \mathrm{ng} / \mathrm{mL})$ for different times was performed and cell extracts were assessed using antibodies that recognized specific phosphorylated residues for TrkA, PLC $\gamma$, MAPK and Akt. Representative western blots are shown $(n=4)$. KI, TrkAP782S; WT, wild-type TrkA. F) Increased survival of DRG neurons from TrkAP782S mice in limiting amounts of NGF. DRG neurons from WT TrkA and TrkAP782S embryos were plated on different amounts of NGF $(25,20,10,5 \mathrm{and} 1 \mathrm{ng} / \mathrm{mL})$. The number of live neurons present in medium supplemented with $25 \mathrm{ng} / \mathrm{mL}$ of NGF was considered $100 \%$ and the rest were normalized with respect to this percentage. Data are presented as means \pm SEM. p-Values were calculated using two-tailed Student's $t$-test $(n=5)$. KI, TrkAP782S; WT, wild-type TrkA. 
$1 \mathrm{ng} / \mathrm{mL}$ ) and survival was monitored on DIV6-9. We observed similar survival percentages in neurons from the WT and $\mathrm{KI}$ when 25, 10 and $5 \mathrm{ng} / \mathrm{mL}$ of NGF were applied to cultured neurons (Figure 7F), although at a concentration of $5 \mathrm{ng} / \mathrm{mL}$ the trend was for there to be lower survival in WT neurons than in $\mathrm{KI}$ neurons (71 versus $99.8 \%$ survival). Using $1 \mathrm{ng} / \mathrm{mL}$ of $\mathrm{NGF}$, the survival of WT neurons was compromised; only $20.9 \%$ of WT neurons survived at $1 \mathrm{ng} / \mathrm{mL}$ in comparison with $100 \%$ in $25 \mathrm{ng} / \mathrm{mL}$ (Figure 7F). In contrast, the percentage of $\mathrm{KI}$ neurons that survived at $1 \mathrm{ng} / \mathrm{mL}$ was very similar to that obtained with $\mathrm{KI}$ neurons cultured with $25 \mathrm{ng} / \mathrm{mL}$ of NGF (Figure 7F). Accordingly, mutant TrkAP782S confers a survival advantage to DRG neurons in limiting amounts of NGF, probably because of the increased TrkA signaling.

\section{Discussion}

Here we provide evidence that Nedd4-2 protein regulates the degradation of TrkA through modulation of the trafficking of the receptor. Depletion of Nedd4-2 caused impairment in the ubiquitination of TrkA in the early endosomes and in the sorting of activated TrkA at the early and late endosomes, the compartments where Nedd4-2 is located, which resulted in enhanced TrkA recycling and retrograde transport. In addition, TrkA signaling and the survival of NGF-dependent DRG neurons were enhanced in response to reduced levels of Nedd4-2. Moreover, neurons from a knock-in mouse expressing a TrkA mutant that does not bind Nedd4-2 behaved in the same way as regards NGF-mediated signaling and the survival effects of Nedd4-2-depleted neurons.

\section{Nedd4-2 regulates TrkA trafficking at the early and late endosome compartments}

The trafficking of activated RTKs is a complex and highly regulated process that modulates the magnitude and controls the specificity of the ligand-elicited response. Alterations of RTK trafficking have profound consequences as regards the amount of receptors and therefore in the final outcome of each specific receptor. Our experiments involving decreasing the levels of Nedd4-2 protein in TrkA-dependent neurons indicate an increase in the levels of TrkA through an alteration of receptor trafficking. Our colocalization and biochemical experiments suggest that activated TrkA in Nedd4-2-depleted neurons arrive at the early endosomes as efficiently as in control neurons. However, once there TrkA is not sorted properly to the late endosomes in Nedd4-2-depleted neurons; instead, there is increased cell-surface recycling and an intracellular accumulation of active TrkA protein. The accumulation of activated TrkA in endosomal compartments has been reported previously when Rab7 activity was impaired (33).

The decision to recycle RTKs is made at the early endosome compartment $(5,6)$. Nedd4-2 depletion leads to increased recycling of TrkA to the plasma membrane (Figure 4A,B) and subsequent accumulation of surface
TrkA (Figures $1 \mathrm{C}$ and $4 \mathrm{D}, \mathrm{E}$ ). As Nedd4-2 is located at the early endosomes (Figure $3 \mathrm{~A}$ ) and considering the delay of activated TrkA in reaching late endosomes when Nedd4-2 is depleted (Figure 3E), we believe that Nedd4-2 must play a role in TrkA trafficking at the early endosomes. In addition, Nedd4-2 is also located in the late endosomes (Figure $3 \mathrm{~A}$ ) and considering the accumulation of activated TrkA at later time-points in late endosomes (Figure 3E), it is very plausible that Nedd4-2 is functioning at the late endosome stage as well. All together, our results indicate that Nedd4-2 is functioning at the early and late endosomal compartments. Nedd4-2 depletion leads to an increased recycling and to a slight accumulation of activated TrkA at the early endosomes in early time-points. At later timepoints upon NGF treatment, Nedd4-2 depletion results in an accumulation of activated TrkA at the late endosomes. To sum up, Nedd4-2 seems to regulate the trafficking and sorting of active TrkA to the degradative pathway acting at the early and late endosome stages. Our data also showed that Nedd4-2 ubiquitinates activated TrkA receptors in early endosomes (Figure $3 \mathrm{G}$ ), suggesting that this may be a mechanism by which TrkA trafficking is regulated. In addition, the effect is specific for TrkA in response to NGF, as p75 trafficking kinetics was not affected. The availability of extra TrkA protein at the cell surface and at intracellular compartments in Nedd4-2-depleted cells renders these neurons more responsive to NGF treatment. This may contribute in part to the increased TrkA signaling observed following NGF treatment (Figure 6A).

Nedd4-2 belongs to the family of Nedd4 E3 ubiquitin ligase proteins $(25,32)$. All members of the family share structural homology, with a C2 domain in the N-terminus, several WW domains and an HECT catalytic domain at the C-terminus $(25,34,35)$. The localization of Nedd4 proteins depends on the C2 domain, which is a phospholipid membrane interaction motif. According to different reports using genetic approaches to knock out their expression, Nedd4 family members exert specific functions (36-40). However, other studies have indicated that there is also functional compensation and redundancy among different Nedd4 proteins $(38,41,42)$. Here, we have described a specific function for Nedd4-2 in the modulation of TrkA trafficking. In the absence of Nedd4-2 TrkA turnover, albeit at lower kinetics, still proceeds, suggesting that other E3 ubiquitin ligases can compensate for Nedd4-2 depletion. Future work should address the role of other members of the Nedd4 family on TrkA protein, mainly those ones that might be upregulated or compensate in response to Nedd4-2 depletion.

The endosomal sorting of RTKs upon activation is initiated by their ubiquitination by specific E3 ubiquitin ligases, which often begins at the plasma membrane and continues on endosomal membranes. In the endosome membrane, ubiquitinated RTKs are recognized and captured by the endosomal sorting complex required for transport machinery. Alteration of the ubiquitination of TrkA mediated by Nedd4-2 impairs the sorting and 
trafficking of the receptor, leading to an accumulation of activated TrkA in compartments where it is not normally accumulated. The level of ubiquitination and/or specific ubiquitination mediated by Nedd4-2 on the TrkA receptor may be required for proper endosomal sorting and degradation, as previously described for EGF receptor (43). Further analysis of the residues that are ubiquitinated in TrkA in response to NGF should shed light on the type and levels of ubiquitination provided by Nedd4-2 that are required for correct TrkA trafficking and sorting.

\section{Implications of alterations of proper TrkA trafficking}

In neurons, TrkA signaling may emanate from the cell surface as well as from intracellular compartments $(4,44)$. There are reports in the literature supporting the notion that activated TrkA exerts its signaling function as from the early endosome $(16,18,45,46)$ as well as from late endosomes $(33,47,48)$. The impaired trafficking of activated TrkA protein in response to Nedd4-2 depletion in sensory neurons results in enhanced signaling, as assessed by the activation of PLC $\gamma$, Akt and MAPK and, finally, in increased survival of these neurons (Figure 6). These data are further supported by the use of sensory neurons obtained from a newly generated knock-in mouse that expresses a mutant TrkA that does not bind Nedd4-2 (Figure 7). These results suggest an important role for Nedd4-2 and its interaction with TrkA in the modulation of NGF-mediated signaling pathways.

The results reported here highlight the notion that correct modulation of the trafficking of the neurotrophin receptor TrkA in response to NGF is a critical event for its functions. It is believed that NGF and TrkA may play important roles in the etiology of neurodegenerative diseases such as Alzheimer's. A hypothesis of impaired NGF transport and the subsequent degeneration of cholinergic neurons may underlie one of the earliest events in Alzheimer's disease (49). Future experiments involving the modulation of TrkA protein levels and NGF-mediated signaling may help to further understand the role of NGF in neurodegenerative diseases. The identification of Nedd4-2 as a molecular determinant of TrkA trafficking represents a potential target for control of the level of TrkA activation.

\section{Materials and Methods}

\section{Materials}

The following antibodies were used: rabbit Nedd4-2 polyclonal and phosphoTrkA (Y785) have been used previously (22); p75 (9992); Trk (C-14) and P4D1 (ubiquitin) (Santa Cruz); Rab7 and blll-Tubulin (Sigma); phosphoTrk (Y490), phosphoPLC $\gamma$ and phosphoMAPK (Cell Signaling); EEA1, Rab5 and Rab11 (Beckton Dickinson); phosphoAkt (Ser473) (Upstate) and GFP (Molecular Probes and Chemicon).

\section{Plasmids}

The sequence 5'-aagtggacaatttaggccgaa-3' of Nedd4-2 cDNA corresponding to nucleotides 727-747 was used to generate Nedd4-2 shRNA using the lentiviral vector pLVTHM. Control shRNA lentivirus was generated using the sequence $5^{\prime}$-gcgcgctttgtaggattcg-3' from Euglena gracilis chloroplast DNA between s16 S and $16 \mathrm{~S}$ rRNA (50).

\section{DRG neuron culture}

DRGs were dissected from E15.5 rat embryos, incubated and dissociated with $0.25 \%$ trypsin in L-15 media for $45 \mathrm{~min}$ at $37^{\circ} \mathrm{C}$. Cells were centrifuged to eliminate trypsin and resuspended in plating medium (MEM, 10\% FBS, $0.4 \%$ glucose, $2 \mathrm{~mm}$ glutamine and $100 \mathrm{U} / \mathrm{mL}$ Pen/Strep). A single-cell suspension was obtained by pipetting up and down 30 times with a $200-\mu \mathrm{L}$ tip. After counting, cells were plated using plating medium and the corresponding neurotrophin, NGF (50 ng/mL) or BDNF ( $25 \mathrm{ng} / \mathrm{mL})$ overnight on plastic plates coated with Growth Factor Reduced Matrigel (BD Biosciences) as substrate. On the following day, the medium was changed to NB (neurobasal medium, B-27, 0.4\% glucose, 2 mm glutamine), NGF or BDNF and 5-fluorodeoxyuridine (5FU; $2.44 \mu \mathrm{g} / \mathrm{mL}$ ) and uridine $(2.44 \mu \mathrm{g} / \mathrm{mL})$. Proliferating cells disappeared after $3-4$ days and more than $95 \%$ of cells at DIV8 were neurons.

\section{Lentivirus generation and infection}

The lentivirus used in this study was generated by cotransfection using calcium phosphate in $293 \mathrm{FT}$ cells. $293 \mathrm{FT}$ cells $\left(2.5 \times 10^{6}\right)$ seeded in a 10 -cm plate the day before were transfected with $9 \mu \mathrm{g}$ of pLVTHM control shRNA or pLVTHM-Nedd4-2 shRNA together with $6 \mu \mathrm{g}$ of psPAX2 and $5 \mu \mathrm{g}$ of pMD.2G plasmids. The medium was changed to fresh medium without antibiotics after $16 \mathrm{~h}$. Forty-eight hours later, the supernatant containing the lentivirus was collected, centrifuged at $2750 \times \mathrm{g}$ for $10 \mathrm{~min}$, filtered through a $0.45 \mu \mathrm{m}$ filter and stored in aliquots at $-80^{\circ} \mathrm{C}$. To infect $1 \times 10^{5}$ DRG cells, $100 \mu \mathrm{L}$ of supernatant containing lentivirus was added to the NB medium. Infected neurons were monitored by the expression of GFP. According to the western blot analysis, Nedd4-2 levels decreased by $80-90 \%$ within $3-4$ days after infection.

\section{Western blot analysis}

Cells were lysed in a lysis buffer (10 mm Tris pH 7.4, $150 \mathrm{~mm} \mathrm{NaCl}, 2 \mathrm{~mm}$ ethylenediaminetetraacetic acid, $1 \%$ Nonidet P-40, 1 mm phenylmethylsulfonyl fluoride, $1 \mu \mathrm{g} / \mathrm{mL}$ aprotinin, $2 \mu \mathrm{g} / \mathrm{mL}$ leueptine, $1 \mathrm{~mm}$ vanadate, $10 \mathrm{~mm}$ sodium fluoride and $20 \mathrm{~mm} \beta$-glycerophosphate) for $40 \mathrm{~min}$ at $4^{\circ} \mathrm{C}$ with gentle shaking and centrifuged at $13000 \times \mathrm{g} 15 \mathrm{~min}$ to eliminate the debris. Lysates were added $5 \times$ SDS buffer and boiled for 7 min to denature the proteins. Proteins were resolved by SDS-PAGE and western blots were performed with antibodies against the different proteins. To avoid problems with the immunoglobulin chains we used ProtA- or ProtG-conjugated horseradish peroxidase when same species antibodies were used for both immunoprecipitation and western blot as previously described (51).

\section{NGF-mediated signaling}

Rat NGF- and BDNF-dependent DRGs at DIV3 were infected with lentivirus expressing control or Nedd4-2 shRNA for 5 days. After overnight starvation of neurotrophins in the presence of $20 \mu \mathrm{M}$ Z-VAD-FMK, DRGs were either not stimulated or stimulated with NGF $(50 \mathrm{ng} / \mathrm{mL})$ for $5,15,30$, 45 and $60 \mathrm{~min}$ at $37^{\circ} \mathrm{C}$ and then immediately chilled on ice. Cells were washed with cold PBS and lysed in $2 \times$ SDS loading buffer (100 mM Tris pH $7.5,25 \%$ glycerol, $2 \%$ SDS and $0.01 \%$ bromophenol blue). Lysates were denatured by heating at $100^{\circ} \mathrm{C}$ for $7 \mathrm{~min}$ and then used for SDS-PAGE. Western blots were performed to analyze pTrk, pAkt Ser473, p-pMAPK, pPLC $\gamma$, Nedd4-2 and $\beta$-tubulin III.

\section{Surface biotinylation assays}

Infected DRGs with control and Nedd4-2 shRNA lentivirus were NGFstarved as indicated above. Cells were stimulated with or without NGF $(50 \mathrm{ng} / \mathrm{mL})$ for different times $(15$ and $60 \mathrm{~min})$ to induce the internalization of biotinylated surface proteins. Subsequently, cells were washed sequentially using room temperature PBS and cold PBS, chilled 
on ice and incubated in $0.5 \mu \mathrm{g} / \mathrm{mL}$ Sulfo-NHS-SS-biotin (Pierce) dissolved in biotinylation buffer (PBS, $1 \mathrm{mM} \mathrm{CaCl}_{2}$ and $0.5 \mathrm{~mm} \mathrm{MgCl}_{2}$ ) for 20 min at $4^{\circ} \mathrm{C}$ to label the membrane proteins. Free biotin was quenched with $0.1 \mathrm{M}$ glycine for 15 min at $4^{\circ} \mathrm{C}$. Cells were then washed twice with cold PBS and lysed as indicated above. Biotinylated proteins were isolated from the total cell lysate by immobilization on neutroavidin beads (Pierce) for at least $3 \mathrm{~h}$ at $4^{\circ} \mathrm{C}$. Beads were washed three times with lysis buffer and $20 \mu \mathrm{L}$ of $2 \times \mathrm{SDS}$ sample buffer was added before boiling for $7 \mathrm{~min}$. Proteins were subjected to SDS-PAGE and immunoblotted with the corresponding antibodies.

\section{Internalization assay}

Unlike the surface biotinylation assay, which tests surface protein after NGF $(50 \mathrm{ng} / \mathrm{mL})$ induction, the internalization assay tested internalized surface protein in response to NGF. Briefly, infected DRG neurons were grown in mass cultures for 8-10 days and then NGF-starved overnight, as indicated previously. Neurons were subjected to biotinylation on ice with the reversible membrane-impermeable derivative of biotin (sulfo-NHS-SSbiotin from Pierce; $0.5 \mathrm{mg} / \mathrm{mL}$ in PBS). Cells were then washed twice to eliminate free biotin and incubated in prewarmed medium at $37^{\circ} \mathrm{C}$ with or without NGF for different times (15 and $60 \mathrm{~min}$ ) to induce the internalization of biotinylated surface protein. To address the rate of internalization, the remaining cell-surface biotin was cleaved by reducing its disulfide linkage with $50 \mathrm{~mm}$ DTT for $15 \mathrm{~min}$ at $4^{\circ} \mathrm{C}$ to assess only the internalized protein. Cells were lysed and biotinylated proteins were precipitated, eluted from the beads, resolved by SDS-PAGE and immunoblotted with the corresponding antibodies.

\section{Degradation assays}

Infected DRGs with control and Nedd4-2 shRNA lentivirus were NGFstarved as indicated above. The following day, neurons were washed with PBS, chilled on ice and biotinylated using $0.5 \mu \mathrm{g} / \mathrm{mL}$ Sulfo-NHS-SS-biotin for $20 \mathrm{~min}$ at $4^{\circ} \mathrm{C}$. Cells were sequentially washed with cold and room temperature PBS and incubated in prewarmed medium at $37^{\circ} \mathrm{C}$ with or without NGF ( $50 \mathrm{ng} / \mathrm{mL}$ ) for different times ( 15 and $60 \mathrm{~min}$ ) to allow the biotinylated receptors to become internalized and degraded. Subsequently, cells were washed and lysed using lysis buffer and the biotinylated proteins were precipitated with neutroavidin beads, washed and subjected to SDS-PAGE and immunoblotted using different antibodies. With this method it was possible to detect surface-labeled proteins that had not been degraded at different time-points, regardless of whether the proteins had been internalized or whether they had returned to the plasma membrane.

\section{Immunofluorescence and quantification}

The DRG neurons used to perform immunofluorescence analysis were cultured on coverslips coated with $1 \mathrm{mg} / \mathrm{mL}$ poly-D-Lysine and Growth Factor Reduced Matrigel and then infected with $10 \mu \mathrm{L}$ of lentivirus expressing control or Nedd4-2 shRNA for 5 days. After NGF starvation as described above, $50 \mathrm{ng} / \mathrm{mL}$ NGF was added to induce TrkA activation and internalization. Cells were then fixed with $4 \%$ paraformaldehyde in PBS for 5 min, quenched with $50 \mathrm{mM} \mathrm{NH}_{4} \mathrm{Cl}$ for $10 \mathrm{~min}$, blocked and permeabilized with PBS containing 5\% normal goat serum, $0.1 \%$ Tween-20, 0.1\% Triton X-100 for $30 \mathrm{~min}$ and incubated with primary antibodies overnight at $4^{\circ} \mathrm{C}$. The following day, cells were washed three times with PBS, incubated with the corresponding fluorescent secondary antibody at room temperature for $40 \mathrm{~min}$ and washed with PBS three times. Images were collected with a Leica confocal microscope. To avoid bleed-through between channels, each channel was acquired separately in the colocalization experiments. For colocalization analyses, images of the cells were processed with the functions of IMAGEJ (National Institutes of Health, NIH), using similar threshold settings for all pictures analyzed, as described (52). Quantification of the processed images was performed with a custom-written program in MATLAB.

\section{Retrograde transport}

DRG neurons were obtained from E.15.5 embryos as described above. Compartmentalized cultures using microfluidic chambers (Standard Neuron
Device $450 \mu \mathrm{m}$, Xona Microfluidics) were used to assess retrograde transport. The microfluidic chambers were assembled on glass coverslips previously treated with $1 \mathrm{mg} / \mathrm{mL}$ PDL the day before dissection of the DRGs, as indicated by the manufacturer. Two hundred microliters of NB media was added to the top wells and $150 \mu \mathrm{L}$ to the bottom wells and incubated overnight in the incubator. The following day, any chamber with signs of leakage was discarded. The chambers were coated with Matrigel and $2 \times 10^{5}$ cells in $10 \mu \mathrm{L}$ of plating medium with NGF were plated in each chamber. Cells were allowed to adhere to the coverslip for $10 \mathrm{~min}$ and additional plating medium was added to each well of the chamber. To isolate each chamber, the volumes in the wells on one side of the device were higher than the other side. The difference in volume creates hydrostatic pressure, thus isolating each compartment fluidically. The plating medium was replaced on the following day with NB medium with NGF $(50 \mathrm{ng} / \mathrm{mL}$ ) and $5 \mathrm{FU}$. Lentivirus infections were performed as described previously. To assess that there was retrograde transport, we have used red-fluorescent beads $(40 \mathrm{~nm}$ ) from Invitrogen.

\section{DRG neuron survival}

NGF- and BDNF-dependent rat DRG neurons plated on 24-well plates were transfected at DIV4-5 with plasmids expressing GFP and control or Nedd42 shRNA using lipofectamine 2000 (Invitrogen) overnight. The following day, the medium was replaced, keeping NGF or BDNF for $48 \mathrm{~h}$, after which both neurotrophins were withdrawn. Transfected cells were recognized by GFP expression and apoptotic neurons, identified by fragmented or condensed nuclei using Hoechst 33342, were scored 72-120 h after the corresponding withdrawal of neurotrophin. Images were taken at random fields using a Leica DMI3000B microscope equipped with a Leica DFC300FX camera. To avoid variability among the independent experiments, a figure of $100 \%$ was given to the percentage of apoptosis after counting all cells.

DRGs from E13.5 WT and KI mice were dissected as indicated above. Ten thousand cells per well in a 24-well plate were plated with $25,10,5,1$ or $0 \mathrm{ng} / \mathrm{mL}$ of NGF. The following day, the medium was changed to NB medium and 5FU, keeping the same amount of NGF as when they had been plated. Images were taken from four random fields after 6-9 days and live cells were counted. The number of live cells growing in $25 \mathrm{ng} / \mathrm{mL}$ of NGF was considered $100 \%$.

\section{TrkAP782S knock-in mice}

To generate a targeting construct for TrkA P782S knock-in mice, a genomic clone from 129SV/J mice containing exon 17, which encodes the C-terminus of TrkA, was used. The triplet coding for proline (CCA) was mutated to serine (TCG) using directed mutagenesis and this was checked by sequencing and the disappearance of a BstXI restriction site. A neomycin-resistance gene flanked by FRT sites containing a loxP site was inserted downstream from exon 17. The truncated diphtheria toxin gene was placed at the end of the short arm for negative selection. The targeting vector was linearized at a unique upstream $B s t X I$ site and was electroporated into CJ7 ES cells, as previously described (53). Ninety-seven G418-resistant colonies were screened for homologous recombination by Southern blot analysis with an external probe. This probe was a $1.2-\mathrm{kb}$ DNA fragment that was excised from the pmA5 plasmid by Sacl digestion. A total of seven clones showed the correct band pattern with the external probe (Figure 7) and a Neo probe (data not shown). Three ES clones were injected into C57BL/6 blastocysts to generate chimeric mice. The chimeras were bred with $\mathrm{C} 57 \mathrm{BL} / 6$ mice that expressed the Flp recombinase driven by the actin promoter to remove the Neo cassette and all three gave germline transmission. TrkAP782S genotyping was performed by PCR with tail genomic DNA and the following two primers: TrkA-Pvul-F (5'ggcgcaggcgatcgagtgtatc- $\left.3^{\prime}\right)$ and mTrkA-1-B (5'-cctccgcaatggacaggag- $\left.3^{\prime}\right)$. A $1.2-\mathrm{kb}$ product was amplified from the WT allele and a $1.4-\mathrm{kb}$ product was amplified from the mutant allele. Confirmation of the genotyping was accomplished by digestion of the PCR product with the BstXI restriction enzyme, which digests the wt alleles but not the mutant alleles. In addition, digestion with the Pstl restriction enzyme was performed to confirm that 
the lack of digestion of mutant TrkA DNA with BstXI was not because of the problems with the amplified DNA.

All animals were housed and bred in the SPF Animal Facility of the University of Salamanca. Proper measures were taken to reduce the pain or discomfort of experimental animals. All animal care and procedures were done in accordance with protocols approved by the Bioethics Committee of the University of Salamanca and following the European Community guidelines.

\section{Acknowledgments}

We thank D. Trono for the lentiviral plasmids; Dionisio Martín-Zanca and Raquel Rodríguez for critical review of the manuscript and helpful discussions and Pilar Pérez for helpful discussions; Enrique LópezPoveda for his help writing the MATLAB custom program to quantify immunofluorescence images. We also thank a reviewer for his/her perseverance helping us to interpret the results. This work was supported by Ministerio de Ciencia e Innovación Grant (BFU2008-00162), by a Marie Curie International Reintegration Grant within the 7th European Community Framework Programme, by Conserjería de Educación (SA074A08) and Conserjeria de Sanidad de Junta Castilla y León to J. C. A. J. C. A. is a 'Ramón y Cajal' Investigator from the University of Salamanca and NARSAD 2009 Young Investigator Awardee. This research was supported by the Intramural Research Program of the NIH, Center for Cancer Research, National Cancer Institute (for E. S. and L. T.).

\section{Supporting Information}

Additional Supporting Information may be found in the online version of this article:

Figure S1: Infection of DRG neurons using lentivirus. Infected DRG neurons were identified by the expression of GFP. Scale bar, $100 \mu \mathrm{m}$.

Figure S2: Nedd4-2 antibodies stain specifically Nedd4-2. DRG neurons infected with control and Nedd4-2 shRNA lentivirus were stained with Nedd4-2 antibodies. Images from control and Nedd4-2-depleted neurons were collected with a confocal microscope using the same settings and processed in a similar way. Note the almost complete disappearance of the signal in Nedd4-2-depleted neurons. Scale bar, $20 \mu \mathrm{m}$.

Figure S3: TrkAP782S receptor binds to PLC $\gamma$. HEK 293 cells were transfected with empty vector (-), wild-type TrkA, TrkAP782S or TrkAY785F. Endogenous PLC $\gamma$ was immunoprecipitated and western blot analysis was performed to test the binding of PLC $\gamma$ to different TrkA receptors. Note the similar binding of wild-type TrkA and TrkAP782S to PLC $\gamma$ and the lack of binding of TrkAY785F.

Please note: Wiley-Blackwell are not responsible for the content or functionality of any supporting materials supplied by the authors. Any queries (other than missing material) should be directed to the corresponding author for the article.

\section{References}

1. Huang EJ, Reichardt LF. Trk receptors: roles in neuronal signal transduction. Annu Rev Biochem 2003;72:609-642.

2. Chao MV. Neurotrophins and their receptors: a convergence point for many signalling pathways. Nat Rev 2003;4:299-309.

3. Vieira AV, Lamaze C, Schmid SL. Control of EGF receptor signaling by clathrin-mediated endocytosis. Science (New York, NY) 1996;274:2086-2089

4. Zhang Y, Moheban DB, Conway BR, Bhattacharyya A, Segal RA. Cell surface Trk receptors mediate NGF-induced survival while internalized receptors regulate NGF-induced differentiation. J Neurosci 2000;20:5671-5678.
5. Sorkin A, von Zastrow M. Endocytosis and signalling: intertwining molecular networks. Nat Rev Mol Cell Biol 2009;10:609-622.

6. Gould GW, Lippincott-Schwartz J. New roles for endosomes: from vesicular carriers to multi-purpose platforms. Nat Rev Mol Cell Biol 2009;10:287-292.

7. Sadowski L, Pilecka I, Miaczynska M. Signaling from endosomes: location makes a difference. Exp Cell Res 2009;315:1601-1609.

8. Knusel B, Gao H, Okazaki T, Yoshida T, Mori N, Hefti F, Kaplan DR. Ligand-induced down-regulation of Trk messenger RNA, protein and tyrosine phosphorylation in rat cortical neurons. Neuroscience 1997;78:851-862.

9. Jullien J, Guili V, Reichardt LF, Rudkin BB. Molecular kinetics of nerve growth factor receptor trafficking and activation. J Biol Chem 2002;277:38700-38708.

10. Saxena $S$, Howe $C L$, Cosgaya JM, Steiner P, Hirling H, Chan JR, Weis J, Kruttgen A. Differential endocytic sorting of p75NTR and TrkA in response to NGF: a role for late endosomes in TrkA trafficking. Mol Cell Neurosci 2005;28:571-587.

11. Sommerfeld MT, Schweigreiter R, Barde YA, Hoppe E. Downregulation of the neurotrophin receptor TrkB following ligand binding. Evidence for an involvement of the proteasome and differential regulation of TrkA and TrkB. J Biol Chem 2000;275:8982-8990.

12. Chen ZY, leraci $A$, Teng $H$, Dall $H$, Meng $C X$, Herrera DG, Nykjaer $A$, Hempstead BL, Lee FS. Sortilin controls intracellular sorting of brainderived neurotrophic factor to the regulated secretory pathway. J Neurosci 2005;25:6156-6166.

13. Ure DR, Campenot RB. Retrograde transport and steady-state distribution of 125I-nerve growth factor in rat sympathetic neurons in compartmented cultures. J Neurosci 1997;17:1282-1290.

14. Kuruvilla R, Ye H, Ginty DD. Spatially and functionally distinct roles of the PI3-K effector pathway during NGF signaling in sympathetic neurons. Neuron 2000;27:499-512.

15. Kuruvilla R, Zweifel LS, Glebova NO, Lonze BE，Valdez G, Ye H, Ginty DD. A neurotrophin signaling cascade coordinates sympathetic neuron development through differential control of TrkA trafficking and retrograde signaling. Cell 2004;118:243-255.

16. Ye H, Kuruvilla R, Zweifel LS, Ginty DD. Evidence in support of signaling endosome-based retrograde survival of sympathetic neurons. Neuron 2003;39:57-68.

17. Ginty DD, Segal RA. Retrograde neurotrophin signaling: Trk-ing along the axon. Curr Opin Neurobiol 2002;12:268-274.

18. Delcroix JD, Valletta JS, Wu C, Hunt SJ, Kowal AS, Mobley WC. NGF signaling in sensory neurons: evidence that early endosomes carry NGF retrograde signals. Neuron 2003;39:69-84.

19. Zweifel LS, Kuruvilla R, Ginty DD. Functions and mechanisms of retrograde neurotrophin signalling. Nat Rev 2005;6:615-625.

20. Hicke L, Dunn R. Regulation of membrane protein transport by ubiquitin and ubiquitin-binding proteins. Annu Rev Cell Dev Biol 2003;19:141-172.

21. Komander $\mathrm{D}$. The emerging complexity of protein ubiquitination. Biochem Soc Trans 2009;37:937-953.

22. Arevalo JC, Waite J, Rajagopal R, Beyna M, Chen ZY, Lee FS, Chao MV. Cell survival through Trk neurotrophin receptors is differentially regulated by ubiquitination. Neuron 2006;50:549-559.

23. Geetha T, Jiang J, Wooten MW. Lysine 63 polyubiquitination of the nerve growth factor receptor TrkA directs internalization and signaling. Mol Cell 2005;20:301-312.

24. Makkerh JP, Ceni C, Auld DS, Vaillancourt F, Dorval G, Barker PA. p75 neurotrophin receptor reduces ligand-induced Trk receptor ubiquitination and delays Trk receptor internalization and degradation. EMBO Rep 2005;6:936-941.

25. Ingham RJ, Gish G, Pawson T. The Nedd4 family of E3 ubiquitin ligases: functional diversity within a common modular architecture. Oncogene 2004;23:1972-1984.

26. Persaud A, Alberts P, Amsen EM, Xiong X, Wasmuth J, Saadon Z, Fladd C, Parkinson J, Rotin D. Comparison of substrate specificity of the ubiquitin ligases Nedd4 and Nedd4-2 using proteome arrays. Mol Syst Biol 2009;5:333.

27. Snider WD. Functions of the neurotrophins during nervous system development: what the knockouts are teaching us. Cell 1994;77:627-638. 


\section{Yu et al.}

28. Marchese A, Raiborg C, Santini F, Keen JH, Stenmark H, Benovic JL. The E3 ubiquitin ligase AIP4 mediates ubiquitination and sorting of the G protein-coupled receptor CXCR4. Dev Cell 2003;5:709-722.

29. Angers A, Ramjaun AR, McPherson PS. The HECT domain ligase itch ubiquitinates endophilin and localizes to the trans-Golgi network and endosomal system. J Biol Chem 2004;279:11471-11479.

30. Rajagopal R, Chen ZY, Lee FS, Chao MV. Transactivation of Trk neurotrophin receptors by G-protein-coupled receptor ligands occurs on intracellular membranes. J Neurosci 2004;24:6650-6658.

31. Chen ZY, leraci A, Tanowitz M, Lee FS. A novel endocytic recycling signal distinguishes biological responses of Trk neurotrophin receptors. Mol Biol Cell 2005; 16:5761-5772.

32. Yang B, Kumar S. Nedd4 and Nedd4-2: closely related ubiquitinprotein ligases with distinct physiological functions. Cell Death Differ 2010;17:68-77

33. Saxena S, Bucci C, Weis J, Kruttgen A. The small GTPase Rab7 controls the endosomal trafficking and neuritogenic signaling of the nerve growth factor receptor TrkA. J Neurosci 2005;25:10930-10940.

34. Harvey KF, Kumar S. Nedd4-like proteins: an emerging family of ubiquitin-protein ligases implicated in diverse cellular functions. Trends Cell Biol 1999;9:166-169.

35. Rotin D, Staub O, Haguenauer-Tsapis R. Ubiquitination and endocytosis of plasma membrane proteins: role of Nedd4/Rsp5p family of ubiquitin-protein ligases. J Membr Biol 2000;176:1-17.

36. Fang D, Elly C, Gao B, Fang N, Altman Y, Joazeiro C, Hunter T, Copeland N, Jenkins N, Liu YC. Dysregulation of $T$ lymphocyte function in itchy mice: a role for Itch in $\mathrm{TH} 2$ differentiation. Nat Immunol 2002;3:281-287.

37. Yang B, Gay DL, MacLeod MK, Cao X, Hala T, Sweezer EM, Kappler J, Marrack P, Oliver PM. Nedd4 augments the adaptive immune response by promoting ubiquitin-mediated degradation of $\mathrm{Cbl}-\mathrm{b}$ in activated T cells. Nat Immunol 2008;9:1356-1363.

38. Yamashita M, Ying SX, Zhang GM, Li C, Cheng SY, Deng CX, Zhang YE. Ubiquitin ligase Smurf1 controls osteoblast activity and bone homeostasis by targeting MEKK2 for degradation. Cell 2005; $121: 101-113$.

39. Shi PP, Cao XR, Sweezer EM, Kinney TS, Williams NR, Husted RF, Nair R, Weiss RM, Williamson RA, Sigmund CD, Snyder PM, Staub O, Stokes JB, Yang B. Salt-sensitive hypertension and cardiac hypertrophy in mice deficient in the ubiquitin ligase Nedd4-2. Am J Physiol 2008;295:F462-F470.

40. Narimatsu M, Bose R, Pye M, Zhang L, Miller B, Ching P, Sakuma R, Luga V, Roncari L, Attisano L, Wrana JL. Regulation of planar cell polarity by Smurf ubiquitin ligases. Cell 2009;137:295-307.
41. Myat A, Henry P, McCabe V, Flintoft L, Rotin D, Tear G. Drosophila Nedd4, a ubiquitin ligase, is recruited by Commissureless to control cell surface levels of the roundabout receptor. Neuron 2002;35:447-459.

42. Wilkin MB, Carbery AM, Fostier M, Aslam H, Mazaleyrat SL, Higgs J, Myat A, Evans DA, Cornell M, Baron M. Regulation of notch endosomal sorting and signaling by Drosophila Nedd4 family proteins. Curr Biol 2004; 14:2237-2244.

43. Huang F, Kirkpatrick D, Jiang X, Gygi S, Sorkin A. Differential regulation of EGF receptor internalization and degradation by multiubiquitination within the kinase domain. Mol Cell 2006;21:737-748.

44. Maclnnis BL, Campenot RB. Retrograde support of neuronal survival without retrograde transport of nerve growth factor. Science (New York, NY) 2002;295:1536-1539.

45. Howe CL, Valletta JS, Rusnak AS, Mobley WC. NGF signaling from clathrin-coated vesicles: evidence that signaling endosomes serve as a platform for the Ras-MAPK pathway. Neuron 2001;32:801-814.

46. Wu C, Lai CF, Mobley WC. Nerve growth factor activates persistent Rap1 signaling in endosomes. J Neurosci 2001;21:5406-5416.

47. Deinhardt K, Salinas S, Verastegui C, Watson R, Worth D, Hanrahan S, Bucci C, Schiavo G. Rab5 and Rab7 control endocytic sorting along the axonal retrograde transport pathway. Neuron 2006;52:293-305.

48. Hisata S, Sakisaka T, Baba T, Yamada T, Aoki K, Matsuda M, Takai Y. Rap1-PDZ-GEF1 interacts with a neurotrophin receptor at late endosomes, leading to sustained activation of Rap1 and ERK and neurite outgrowth. J Cell Biol 2007;178:843-860.

49. Calissano P, Matrone C, Amadoro G. Nerve growth factor as a paradigm of neurotrophins related to Alzheimer's disease. Dev Neurobiol 2010;70:372-383.

50. Kuratomi G, Komuro A, Goto K, Shinozaki M, Miyazawa K, Miyazono K, Imamura T. NEDD4-2 (neural precursor cell expressed, developmentally down-regulated 4-2) negatively regulates TGF-beta (transforming growth factor-beta) signalling by inducing ubiquitin-mediated degradation of Smad2 and TGF-beta type I receptor. Biochem J 2005;386:461-470.

51. Lal A, Haynes SR, Gorospe M. Clean western blot signals form immunoprecipitated samples. Mol Cell Probes 2005;19:385-388.

52. Tai CY, Mysore SP, Chiu C, Schuman EM. Activity-regulated Ncadherin endocytosis. Neuron 2007;54:771-785.

53. Tessarollo L. Manipulating mouse embryonic stem cells. Methods Mol Biol 2001;158:47-63. 